\title{
EFfects of Spacing And GeOMetry of DisTRIBUTED ROUGHNESS ELEMENTS ON A TWo-Dimensional Turbulent Boundary LAYER
}

by Devin O. Stewart

\author{
Thesis submitted to the Faculty of the \\ Virginia Polytechnic Institute and State University \\ in partial fulfillment of the requirements for the degree of
}

Master of Science

in

Aerospace Engineering

Dr. Roger Simpson, Chair

Dr. William Devenport

Dr. Wayne Neu

26 August 2005

Blacksburg, Virginia

Keywords: Distributed roughness, Laser Doppler Velocimetry, Turbulent boundary layer, Near-wall flow structure, Reynolds stresses, Triple products Copyright 2005, Devin O. Stewart 


\title{
Abstract \\ Effects of Spacing and GeOMetry of Distributed Roughness ElEMENTS ON A TWO-Dimensional TuRbulent Boundary LAYER
}

\author{
by Devin O. Stewart
}

This thesis is a study of the effects of distributed roughness elements on a two-dimensional turbulent boundary layer. Measurements were taken on a total of ten rough wall configurations: four involving Gaussian spikes, and six with circular cylindrical posts. Rough wall flows are particularly suited to study with Laser Doppler Velocimetry (LDV) due to the fact that measurements are required near a solid surface, as well has in highly turbulent fluid. The LDV system used in this study is a fine resolution $(\approx 50 \mu \mathrm{m})$, threecomponent, fiber optic system. All mean velocities, Reynolds stresses, and triple products are measured. This study is unique in the range and variety of roughness cases for which data was taken.

The data show that the flow over a rough wall is characterized by high levels of turbulence near the roughness element peaks at the interface between low-speed, near-wall fluid and the higher speed fluid above. Behind an element, high-momentum fluid sweeps toward the wall, and there is a small region of ejection of low-momentum fluid. Cylindrical elements typically have larger magnitudes of turbulent stresses at their peaks compared to Gaussian elements. Trends in mean velocity profile parameters such as displacement height, roughness effect, and wake parameter are examined with respect to roughness element geometry and spacing. 


\section{Acknowledgments}

I would first and foremost like to thank my parents for their support throughout my education. Thanks also go to the friends and family with whom I've been fortunate enough to share the experience of graduate school.

Numerous people have been instrumental to the completion of this research. The guidance and experience of my advisor, Dr. Roger Simpson, has been indispensable. I would also like to thank the other members of my committee, Dr. William Devenport and Dr. Wayne Neu for their aid and input on this thesis.

My fellow students and co-workers have helped me enormously throughout my time at Virginia Tech. I would like to thank Jeremy Bennington and $\mathrm{K}$. Todd Lowe for their assistance in learning the operation of the LDV system, Gwibo Byun for his help with the data acquisition and reduction software, and Qing Tian and Edgar Orsi for their help in fabricating the roughness fetches. All of the students in the research group have been generous with their aid and advice, and have helped me on occasions too numerous to list here. My fondest memories of graduate school will be of working with such a good-natured and dedicated group of students.

I would also like to thank Steve Edwards, James Lambert, Bruce Stanger, and Mike Vaught, whose day-to-day assistance in the lab was critical to this research. The AOE office staff, Gail Coe, Wanda Foushee, and Betty Williams, were also of great help.

Finally, thanks to the Office of Naval Research and Program Manager Dr. Ronald Joslin. This work was supported under ONR Grant N00014-01$1-0421$. 


\section{Contents}

Acknowledgments

Table of Contents iv

List of Figures $\quad$ XV

$\begin{array}{ll}\text { List of Tables } & \text { xvi }\end{array}$

$\begin{array}{ll}\text { Nomenclature } & \text { xvii }\end{array}$

1 Introduction 1

1.1 Motivation ..................... 1

1.2 Rough Wall Turbulent Boundary Layers . . . . . . . . . . . 2

1.3 The Law of the Wall for Rough Walls . . . . . . . . . . . . . 3

1.4 Effects of Spacing and Geometry . . . . . . . . . . 3

1.5 Roughness and the Law of the Wake . . . . . . . . . . 5

1.6 Displacement Height . . . . . . . . . . . . . . . . 6

1.7 Transitional Roughness . . . . . . . . . . . . . . 6

1.8 Overview of Thesis . . . . . . . . . . . . . 7 
2 Apparatus and Instrumentation $\quad 8$

2.1 Small Boundary Layer Wind Tunnel . . . . . . . . . . . . 8

2.1.1 Tunnel Overview . . . . . . . . . . . . . . . 8

2.1.2 Flow Conditions . . . . . . . . . . . . . . 11

2.1.3 Seeding System . . . . . . . . . . . . . . 13

2.2 LDV System . . . . . . . . . . . . . . . . 13

2.2.1 Optical Table .................. 13

2.2.2 Probe Head . . . . . . . . . . . . . . . . . . . 15

2.2.3 Signal Conditioning \& Data Acquisition System . . . . 15

2.2.4 Data Processing . . . . . . . . . . . . 20

2.3 Outer layer LDV System . . . . . . . . . . . . . . 21

2.4 Traverse and Location of Wall . . . . . . . . . . . . . . . . . . 21

2.5 Roughness Fetches . . . . . . . . . . . . . . . . . 23

2.5.1 Description .................. 24

2.5.2 Construction ................. . . 30

2.5.3 Determination of Origin . . . . . . . . . . 31

3 Results 33

3.1 Determination of Friction Velocity . . . . . . . . . . 33

3.2 Mean Velocities . . . . . . . . . . . . . . . 38

3.3 Reynolds Stresses and Turbulent Kinetic Energy . . . . . . . . 44

3.4 Triple Products . . . . . . . . . . . . . . . 51

4 Discussion and Analysis $\quad 56$

4.1 Introduction . . . . . . . . . . . . . . . . 56

4.2 Summary of Rough Wall Flow Behavior . . . . . . . . . . 56 
4.3 Boundary Layer Characteristics . . . . . . . . . . . . . . 59

4.4 Determination of Displacement Height . . . . . . . . . . . 60

4.5 Wake Parameter . . . . . . . . . . . . . 66

4.6 Roughness Correlations . . . . . . . . . . . . . 67

$\begin{array}{lll}5 & \text { Conclusions \& Future Work } & 77\end{array}$

5.1 Summary of Study . . . . . . . . . . . . . . . 77

5.2 Conclusions . . . . . . . . . . . . . . . . . 78

5.3 Future Work . . . . . . . . . . . . . . . 80

$\begin{array}{lr}\text { Bibliography } & 81\end{array}$

$\begin{array}{ll}\text { A Complete Data Plots } & 87\end{array}$

A.1 Gaussian Spikes, 5.49 mm Spacing, Staggered . . . . . . . . 88

A.2 Gaussian Spikes, 8.23 mm Spacing, Straight . . . . . . . . 95

A.3 Gaussian Spikes, 8.23 mm Spacing, Staggered . . . . . . . . . 102

A.4 0.38 mm Cylinders, Straight . . . . . . . . . . . 109

A.5 0.38 mm Cylinders, Staggered . . . . . . . . . . 116

A.6 0.76 mm Cylinders, Straight . . . . . . . . . . 123

A.7 0.76 mm Cylinders, Staggered . . . . . . . . . . 130

A.8 $1.52 \mathrm{~mm}$ Cylinders, Straight . . . . . . . . . . . 137

A.9 1.52 mm Cylinders, Staggered . . . . . . . . . . . 144

B Uncertainty Analysis 151

B.1 Wall Location . . . . . . . . . . . . . . . . . . . 151

B.2 Horizontal Measurement Location . . . . . . . . . . . . . 151

B.3 Free-stream velocity . . . . . . . . . . . . . . . 152 
B.4 Velocities . . . . . . . . . . . . . . . . . 152

B.5 Skin Friction Velocity . . . . . . . . . . . . . . 154

B.6 Turbulent Kinetic Energy _ . . . . . . . . . . . . . . . 154

C Monodisperse Seeding Study 156

C.1 Introduction . . . . . . . . . . . . . . 156

C.2 Seeder Output Requirements . . . . . . . . . . . . . 157

C.3 Polystrene Latex (PSL) Sphere Seeding . . . . . . . . . . . 160

C.3.1 Injection of Seed into Tunnel . . . . . . . . . . . . . . 160

C.3.2 Spray Systems Nozzles . . . . . . . . . . . . . . . 162

C.3.3 Six-Jet Atomizer . . . . . . . . . . . . . . . . . . 165

C.3.4 Laskin Nozzle . . . . . . . . . . . . . . . . . . . 167

C.3.5 Ultrasonic Nozzles . . . . . . . . . . . . . . . . 168

C.3.6 Medical Nebulizers . . . . . . . . . . . . . . . . 169

C.4 DOP Seeding with a Vap/Con Aerosol Generator . . . . . . . 175

C.4.1 Design Details . . . . . . . . . . . . . . . . . 177

C.4.2 Results . . . . . . . . . . . . . . . . . 178

C.5 Conclusions . . . . . . . . . . . . . . . . 179

$\begin{array}{ll}\text { Vita } & 181\end{array}$ 


\section{List of Figures}

2.1 Virginia Tech Small Boundary Layer Wind Tunnel _. . . . 9

2.2 Schematic of wind tunnel . . . . . . . . . . . . . . 10

2.3 Spanwise traverse of wind tunnel test section at mid-height . . 12

2.4 Smooth Wall LDV profiles, along with Spalart (1988) DNS data . . . . . . . . . . . . . . . . . 14

2.5 Photograph of the LDV optical table . . . . . . . . . 16

2.6 Optical table components at exit of laser . . . . . . . . . 17

2.7 Photograph of LDV probe head . . . . . . . . . . . 17

2.8 LDV probe head while taking data $\ldots \ldots \ldots \ldots$

2.9 Outer Layer LDV System _ . . . . . . . . . . . . . . 22

2.10 Photographs of roughness fetches $\ldots \ldots \ldots \ldots$

2.11 Roughness fetch installed in wind tunnel . . . . . . . . . 26

2.12 LDV measurements being taken through slot in roughness . . 27

2.13 Example measurement locations for a roughness fetch in a straight configuration . . . . . . . . . . . 28

2.14 Example measurement locations for a roughness fetch in a staggered configuration . . . . . . . . . . . . . . . . . 29

3.1 Control volume for calculation of $u_{\tau} \ldots \ldots \ldots \ldots$

3.2 Comparison of spatially averaged mean velocity profiles . . . 38 
3.3 Example centerline streamwise mean velocities . . . . . . . . . 40

3.4 Example mid-plane streamwise mean velocities . . . . . . . . . 41

3.5 Example centerline vertical mean velocities . . . . . . . . . . 42

3.6 Example mid-plane spanwise mean velocities . . . . . . . . . 43

3.7 Example centerline $\overline{u^{2}} / u_{\tau}^{2}$ normal stresses . . . . . . . . . . . 45

3.8 Example centerline $\overline{v^{2}} / u_{\tau}^{2}$ and $\overline{w^{2}} / u_{\tau}^{2}$ normal stresses . . . . . 46

3.9 Example mid-plane $\overline{v^{2}} / u_{\tau}^{2}$ and $\overline{w^{2}} / u_{\tau}^{2}$ normal stresses . . . . . 47

3.10 Example centerline $\overline{u v} / u_{\tau}^{2}$ shear stresses . . . . . . . . . . . . . 48

3.11 Example mid-plane TKE data . . . . . . . . . . . . . . . . . . 49

3.12 Mid-plane $\overline{u^{3}} / u_{\tau}^{3}$ and $\overline{u^{2} v} / u_{\tau}^{3}$ for $0.76 \mathrm{~mm}$ cylinder, straight . . 51

3.13 Mid-plane $\overline{u^{3}} / u_{\tau}^{3}$ and $\overline{u^{2} v} / u_{\tau}^{3}$ for $8.23 \mathrm{~mm}$ spacing, Gauss, straight . . . . . . . . . . . . . . . . 52

3.14 Mid-plane $\overline{v^{3}} / u_{\tau}^{3}, \overline{u v^{2}} / u_{\tau}^{3}$ for $8.23 \mathrm{~mm}$ spacing, Gauss, straight 53

3.15 Mid-plane $\overline{v^{3}} / u_{\tau}^{3}, \overline{u v^{2}} / u_{\tau}^{3}$ for $8.23 \mathrm{~mm}$ spacing, Gauss, straight 55

4.1 Illustration of flow near roughness elements . . . . . . . . . . 57

4.2 Illustration of displacement height . . . . . . . . . . . . 61

4.3 Geometric Model of Separation Volume . . . . . . . . . . 63

4.4 Determination of downstream separation volume with linear fit to experimental velocity data . . . . . . . . . . . . 64

4.5 Plot of wake parameter $\Pi$ versus scale ratio $k / \delta$. . . . . 68

4.6 Plot of $f(\lambda)$ vs. $\lambda^{-1} \ldots \ldots \ldots 70$

4.7 Plot of $U^{+}$versus $k+$ with estimated lines of constant $\lambda . \quad$. . 73

4.8 Plot of $f(\lambda)$ vs. $\lambda^{-1}$, with functional relationship defined in Equation 4.18. . . . . . . . . . . . . . . . . . . . . . . . . 74

4.9 Plot of $f(\lambda)$ vs. $\lambda^{-1}$, with data of additional authors. . . . 75 
A.1 $5.49 \mathrm{~mm}$ spaced staggered Gaussian spikes: Mean velocity and Reynolds stress centerline plots . . . . . . . . . . . . 88

A.2 $5.49 \mathrm{~mm}$ spaced staggered Gaussian spikes: Triple product centerline plots . . . . . . . . . . . . . . . . . . . 89

A.3 $5.49 \mathrm{~mm}$ spaced staggered Gaussian spikes: Turbulent kinetic energy and structure parameters $a_{1}$ and $1 / S$ centerline plots . 90

A.4 $5.49 \mathrm{~mm}$ spaced staggered Gaussian spikes: Mean velocity and Reynolds normal stress mid-plane plots . . . . . . . . . . . . 91

A.5 $5.49 \mathrm{~mm}$ spaced staggered Gaussian spikes: Reynolds shear stress and triple product mid-plane plots . . . . . . . . . . . 92

A.6 $5.49 \mathrm{~mm}$ spaced staggered Gaussian spikes: Triple product mid-plane plots (continued) . . . . . . . . . . . . . . 93

A.7 $5.49 \mathrm{~mm}$ spaced staggered Gaussian spikes: $\overline{u v w} / u_{\tau}^{2}$ triple product, turbulent kinetic energy and structure parameters $a_{1}$ and $1 / S$ mid-plane plots $\ldots \ldots \ldots . \ldots 94$

A.8 $8.23 \mathrm{~mm}$ spaced straight Gaussian spikes: Mean velocity and Reynolds stress centerline plots . . . . . . . . . . . . . 95

A.9 $8.23 \mathrm{~mm}$ spaced straight Gaussian spikes: Triple product centerline plots . . . . . . . . . . . . . . . 96

A.10 $8.23 \mathrm{~mm}$ spaced straight Gaussian spikes: Turbulent kinetic energy and structure parameters $a_{1}$ and $1 / S$ centerline plots . 97

A.11 $8.23 \mathrm{~mm}$ spaced straight Gaussian spikes: Mean velocity and Reynolds normal stress mid-plane plots . . . . . . . . . . 98

A.12 $8.23 \mathrm{~mm}$ spaced straight Gaussian spikes: Reynolds shear stress and triple product mid-plane plots . . . . . . . . . . . 99

A.13 $8.23 \mathrm{~mm}$ spaced straight Gaussian spikes: Triple product midplane plots (continued) . . . . . . . . . . . . . . . 100

A.14 $8.23 \mathrm{~mm}$ spaced straight Gaussian spikes: $\overline{u v w} / u_{\tau}^{2}$ triple product, turbulent kinetic energy and structure parameters $a_{1}$ and $1 / S$ mid-plane plots . . . . . . . . . . . . . . . . . . 101 
A.15 $8.23 \mathrm{~mm}$ spaced staggered Gaussian spikes: Mean velocity and Reynolds stress centerline plots . . . . . . . . . . . . . 102

A.16 $8.23 \mathrm{~mm}$ spaced staggered Gaussian spikes: Triple product centerline plots . . . . . . . . . . . . . . . . . 103

A.17 $8.23 \mathrm{~mm}$ spaced staggered Gaussian spikes: Turbulent kinetic energy and structure parameters $a_{1}$ and $1 / S$ centerline plots . 104

A.18 $8.23 \mathrm{~mm}$ spaced staggered Gaussian spikes: Mean velocity and Reynolds normal stress mid-plane plots _ . . . . . . . . . 105

A.19 $8.23 \mathrm{~mm}$ spaced staggered Gaussian spikes: Reynolds shear stress and triple product mid-plane plots . . . . . . . . . 106

A.20 $8.23 \mathrm{~mm}$ spaced staggered Gaussian spikes: Triple product mid-plane plots (continued) . . . . . . . . . . . . . 107

A.21 $8.23 \mathrm{~mm}$ spaced staggered Gaussian spikes: $\overline{u v w} / u_{\tau}^{2}$ triple product, turbulent kinetic energy and structure parameters $a_{1}$ and $1 / S$ mid-plane plots . . . . . . . . . . . . 108

A.22 $0.38 \mathrm{~mm}$ Cylinders, straight: Mean velocity and Reynolds stress centerline plots . . . . . . . . . . . . . . . 109

A.23 $0.38 \mathrm{~mm}$ Cylinders, straight: Triple product centerline plots . 110

A.24 $0.38 \mathrm{~mm}$ Cylinders, straight: Turbulent kinetic energy and structure parameters $a_{1}$ and $1 / S$ centerline plots $\ldots \ldots . .111$

A.25 $0.38 \mathrm{~mm}$ Cylinders, straight: Mean velocity and Reynolds normal stress mid-plane plots . . . . . . . . . . . . . . . . 112

A.26 $0.38 \mathrm{~mm}$ Cylinders, straight: Reynolds shear stress and triple product mid-plane plots . . . . . . . . . . . . . . . . 113

A.27 $0.38 \mathrm{~mm}$ Cylinders, straight: Triple product mid-plane plots (continued) . . . . . . . . . . . . . . . . . . . 114

A.28 $0.38 \mathrm{~mm}$ Cylinders, straight: $\overline{u v w} / u_{\tau}^{2}$ triple product, turbulent kinetic energy and structure parameters $a_{1}$ and $1 / S$ mid-plane plots . . . . . . . . . . . . . . . . . 115 
A.29 $0.38 \mathrm{~mm}$ Cylinders, staggered: Mean velocity and Reynolds stress centerline plots . . . . . . . . . . . . 116

A.30 $0.38 \mathrm{~mm}$ Cylinders, staggered: Triple product centerline plots 117

A.31 $0.38 \mathrm{~mm}$ Cylinders, staggered: Turbulent kinetic energy and structure parameters $a_{1}$ and $1 / S$ centerline plots $\ldots \ldots . .118$

A.32 $0.38 \mathrm{~mm}$ Cylinders, staggered: Mean velocity and Reynolds normal stress mid-plane plots . . . . . . . . . . . . . . 119

A.33 $0.38 \mathrm{~mm}$ Cylinders, staggered: Reynolds shear stress and triple product mid-plane plots . . . . . . . . . . . . . . . 120

A.34 $0.38 \mathrm{~mm}$ Cylinders, staggered: Triple product mid-plane plots (continued) . . . . . . . . . . . . . . . 121

A.35 $0.38 \mathrm{~mm}$ Cylinders, staggered: $\overline{u v w} / u_{\tau}^{2}$ triple product, turbulent kinetic energy and structure parameters $a_{1}$ and $1 / S$ mid-plane plots . . . . . . . . . . . . . . . . 122

A.36 $0.76 \mathrm{~mm}$ Cylinders, straight: Mean velocity and Reynolds stress centerline plots . . . . . . . . . . . . . . . 123

A.37 $0.76 \mathrm{~mm}$ Cylinders, straight: Triple product centerline plots . 124

A.38 $0.76 \mathrm{~mm}$ Cylinders, straight: Turbulent kinetic energy and structure parameters $a_{1}$ and $1 / S$ centerline plots $\ldots \ldots 125$

A.39 $0.76 \mathrm{~mm}$ Cylinders, straight: Mean velocity and Reynolds normal stress mid-plane plots . . . . . . . . . . . . . . 126

A.40 $0.76 \mathrm{~mm}$ Cylinders, straight: Reynolds shear stress and triple product mid-plane plots . . . . . . . . . . . . . . 127

A.41 $0.76 \mathrm{~mm}$ Cylinders, straight: Triple product mid-plane plots

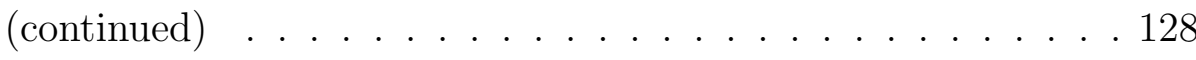

A.42 $0.76 \mathrm{~mm}$ Cylinders, straight: $\overline{u v w} / u_{\tau}^{2}$ triple product, turbulent kinetic energy and structure parameters $a_{1}$ and $1 / S$ mid-plane plots . . . . . . . . . . . . . . . . . . . 129

A.43 $0.76 \mathrm{~mm}$ Cylinders, staggered: Mean velocity and Reynolds stress centerline plots . . . . . . . . . . . . . 130 
A.44 0.76 mm Cylinders, staggered: Triple product centerline plots 131

A.45 $0.76 \mathrm{~mm}$ Cylinders, staggered: Turbulent kinetic energy and structure parameters $a_{1}$ and $1 / S$ centerline plots . . . . . . 132

A.46 $0.76 \mathrm{~mm}$ Cylinders, staggered: Mean velocity and Reynolds normal stress mid-plane plots . . . . . . . . . . . . . . 133

A.47 0.76 mm Cylinders, staggered: Reynolds shear stress and triple product mid-plane plots . . . . . . . . . . . . . . . . . . 134

A.48 $0.76 \mathrm{~mm}$ Cylinders, staggered: Triple product mid-plane plots (continued) . . . . . . . . . . . . . . 135

A.49 $0.76 \mathrm{~mm}$ Cylinders, staggered: $\overline{u v w} / u_{\tau}^{2}$ triple product, turbulent kinetic energy and structure parameters $a_{1}$ and $1 / S$ mid-plane plots . . . . . . . . . . . . . . . 136

A.50 $1.52 \mathrm{~mm}$ Cylinders, straight: Mean velocity and Reynolds stress centerline plots . . . . . . . . . . . . . 137

A.51 $1.52 \mathrm{~mm}$ Cylinders, straight: Triple product centerline plots . 138

A.52 $1.52 \mathrm{~mm}$ Cylinders, straight: Turbulent kinetic energy and structure parameters $a_{1}$ and $1 / S$ centerline plots . . . . . . 139

A.53 $1.52 \mathrm{~mm}$ Cylinders, straight: Mean velocity and Reynolds normal stress mid-plane plots . . . . . . . . . . . . . . . . . . 140

A.54 $1.52 \mathrm{~mm}$ Cylinders, straight: Reynolds shear stress and triple product mid-plane plots . . . . . . . . . . . . . . . . . . 141

A.55 $1.52 \mathrm{~mm}$ Cylinders, straight: Triple product mid-plane plots (continued) . . . . . . . . . . . . . . . 142

A.56 $1.52 \mathrm{~mm}$ Cylinders, straight: $\overline{u v w} / u_{\tau}^{2}$ triple product, turbulent kinetic energy and structure parameters $a_{1}$ and $1 / S$ mid-plane plots . . . . . . . . . . . . . . . . . . . 143

A.57 $1.52 \mathrm{~mm}$ Cylinders, staggered: Mean velocity and Reynolds stress centerline plots . . . . . . . . . . . . . . . . 144

A.58 $1.52 \mathrm{~mm}$ Cylinders, staggered: Triple product centerline plots 145 
A.59 $1.52 \mathrm{~mm}$ Cylinders, staggered: Turbulent kinetic energy and structure parameters $a_{1}$ and $1 / S$ centerline plots $\ldots \ldots 146$

A.60 $1.52 \mathrm{~mm}$ Cylinders, staggered: Mean velocity and Reynolds normal stress mid-plane plots . . . . . . . . . . . . . . 147

A.61 $1.52 \mathrm{~mm}$ Cylinders, staggered: Reynolds shear stress and triple product mid-plane plots . . . . . . . . . . . . . . . . . 148

A.62 $1.52 \mathrm{~mm}$ Cylinders, staggered: Triple product mid-plane plots (continued) . . . . . . . . . . . . . . . . . . 149

A.63 $1.52 \mathrm{~mm}$ Cylinders, staggered: $\overline{u v w} / u_{\tau}^{2}$ triple product, turbulent kinetic energy and structure parameters $a_{1}$ and $1 / S$ mid-plane plots . . . . . . . . . . . . . . 150

C.1 $2.7 \mu m$ PSL spheres magnified 2000x . . . . . . . . . 158

C.2 Representative histogram of particle size distribution produced by a Laskin nozzle seeder . . . . . . . . . . . . . . . . . . . 159

C.3 Seed injection apparatus . . . . . . . . . . . . . . . 161

C.4 Baffle plate . . . . . . . . . . . . . . . . . . 161

C.5 Schematic of Spray Systems nozzle apparatus . . . . . . . 162

C.6 Spray Systems nozzle assembly, mounted inside large plenum 163

C.7 Large plenum box . . . . . . . . . . . . . . . . . . 165

C.8 TSI Six-Jet Atomizer drawing . . . . . . . . . . . . 166

C.9 Schematic of ultrasonic nozzle . . . . . . . . . . . . . 169

C.10 Invacare Sidestream nebulizer _ . . . . . . . . . . . 170

C.11 Supply pressure vs. nebulizer output flowrate . . . . . . . . 172

C.12 Nebulization rate vs. supply pressure . . . . . . . . . . . 173

C.13 Nebulizer float . . . . . . . . . . . . . . . . . . . . . 173

C.14 Nebulizer seeder . . . . . . . . . . . . . . . . . . . . 174

C.15 Vap/con Generator Schematic and Photograph . . . . . . . . 176 
C.16 Time series with and without vap/con . . . . . . . . . 179 


\section{List of Tables}

2.1 Roughness fetch configurations _. . . . . . . . . . . 23

2.2 Average Percent Difference in Upstream and Downstream Velocity Profiles . . . . . . . . . . . . . . . 30

$3.1 u_{\tau}$ and $k^{+}$values $\ldots \ldots \ldots \ldots \ldots \ldots \ldots \ldots$

4.1 Boundary layer thickness $\delta, k / \delta$, Wake parameter $\Pi$, and momentum thickness Reynolds number $R e_{\theta}$. . . . . . . . . . . 61

4.2 Normalized displacement height as calculated by fit to Cole's Law of the Wall and by geometric method . . . . . . . . . . 65

4.3 Normalized displacement height as calculated by the geometric method, compared with curve fit values of George (2005) . . . 66

4.4 Roughness effect $\Delta U / u_{\tau}$ and $f(\lambda) \quad \ldots \ldots \ldots$

B.1 Velocity, Reynolds stress, and triple product uncertainties with $21: 1$ odds . . . . . . . . . . . . . . . . 153

B.2 Skin friction velocity uncertainties with $20: 1$ odds $\ldots \ldots . .154$ 
Nomenclature 


\begin{tabular}{ll}
\hline$\underline{\text { Roman }} 1 / S$ & Turbulence structure parameter, $-\overline{u v} / \overline{v^{2}}$ \\
$a_{1}$ & Turbulence structure parameter, $-\overline{u v} / \overline{q^{2}}$ \\
$A_{F}$ & Total projected frontal area of roughness elements \\
$A_{M V}$ & Area of the measurement volume \\
$A_{S}$ & Total wall surface area \\
$C$ & Seed particle concentration \\
$C_{B L, 1}$ & Particle concentration in boundary layer due to one nebulizer \\
$C_{s o l n}$ & Particle concentration in PSL solution \\
$C L$ & Centerline \\
$C V$ & Control volume \\
$d$ & Roughness element base diameter \\
$d_{m a x}$ & Average of half the differences of two quantities \\
$\mathrm{d} A$ & Differential area \\
$\mathrm{d} \sigma$ & Differential volume \\
$D$ & Roughness element spacing \\
$\mathrm{DOP}$ & di-octyl phthalate \\
$\mathrm{F}_{x}$ & Force acting in the streamwise direction \\
$g$ & Acceleration due to gravity \\
$h_{D S}$ & Length of separation downstream of roughness element \\
$h_{m}$ & Manometer reading \\
$h_{U S}$ & Length of separation upstream of roughness element \\
$\hat{i}$ & Unit vector in the streamwise direction \\
$k$ & Roughness element height \\
$k_{s}$ & Sand grain height \\
$k^{+}$ & Non-dimensional roughness element height, $k u_{\tau} / \nu$ \\
$L$ & Streamwise spacing of two-dimensional roughness elements \\
$L D V$ & Laser Doppler Velocimetry \\
$n$ & Number of samples \\
$\hat{n}$ & Unit vector normal to a control volume surface \\
&
\end{tabular}




\begin{tabular}{|c|c|}
\hline$p$ & Static pressure \\
\hline$P I V$ & Particle Image Velocimetry \\
\hline$P S L$ & Polystyrene Latex \\
\hline$Q_{B L}$ & Volumetric flow rate of the boundary layer \\
\hline$Q_{l i q}$ & Liquid flow rate \\
\hline$Q_{M V}$ & Flow rate through measurement volume \\
\hline$Q_{n e b}$ & Nebulization rate \\
\hline$\overline{q^{2}}$ & $\left(\overline{u^{2}}+\overline{w^{2}}+\overline{v^{2}}\right)$ \\
\hline$r$ & Rate of seed particle production \\
\hline$r_{n e b}$ & Rate of seed particle production by nebulizer \\
\hline$R$ & Residual \\
\hline$R e_{\theta}$ & Momentum thickness Reynolds number, $U_{\infty} \theta / \nu$ \\
\hline$R e_{x}$ & Reynolds number, $U_{\infty} x / \nu$ \\
\hline$s$ & Streamwise dimension of a roughness element \\
\hline$S$ & Curve fit parameter in Equation 4.18 \\
\hline$t_{4 \text { part }}$ & Time for 4 particles to arrive in the measurement volume \\
\hline TKE & Turbulent Kinetic Energy, $\left(\overline{u^{2}}+\overline{w^{2}}+\overline{v^{2}}\right) / 2$ \\
\hline$u_{\tau}$ & Skin Friction velocity: $\sqrt{\tau / \rho}$ \\
\hline$U_{\text {rep }}$ & Representative velocity used in seeding calculations \\
\hline$U_{\infty}$ & Free-stream Velocity \\
\hline$u, v, w$ & $\begin{array}{l}\text { Instantaneous fluctuating velocity components in tunnel coordi- } \\
\text { nates }\end{array}$ \\
\hline$U, V, W$ & $\begin{array}{l}\text { Statistical mean velocity from the selected ensemble in tunnel } \\
\text { co-ordinates }\end{array}$ \\
\hline$U^{+}, V^{+}, W^{+}$ & Non-dimensional mean velocities, $U / u_{\tau}, V / u_{\tau}, W / u_{\tau}$ \\
\hline$\overline{u^{2}}, \overline{v^{2}}, \overline{w^{2}}$ & Reynolds normal stresses \\
\hline$-\overline{u v},-\overline{u w},-\overline{v w}$ & Reynolds shear stresses \\
\hline$\overline{u^{2} v}, \overline{v w^{2}}, \overline{v^{3}}$ & Statistical mean triple products \\
\hline
\end{tabular}




\begin{tabular}{ll}
$V_{D S}$ & Volume of separation region downstream of roughness element \\
$V_{e}$ & Roughness element volume \\
$V_{q}$ & Normal-to-wall diffusion velocity, $\overline{v q^{2}} / \overline{q^{2}}$ \\
$V_{U S}$ & Volume of separation region upstream of roughness element \\
$W$ & Test section width \\
$W_{q}$ & Spanwise diffusion velocity, $\overline{w q^{2}} / \overline{q^{2}}$ \\
$x, y, z$ & Wind tunnel coordinate system \\
$y^{\prime}$ & Distance above wall, without correction of displacement height \\
$y^{+}$ & Non-dimensional distance from wall, yu $/ \nu$ \\
\hline Greek & \\
$\delta$ & Boundary layer thickness, $\delta=$ y when $\frac{U}{U_{\infty}}=0.99$ \\
$\delta^{*}$ & Displacement thickness \\
$\delta \mathrm{x}$ & Uncertainty in quantity $x$ \\
$\Delta U / u_{\tau}$ & Roughness effect, shift of logarithmic velocity profile \\
$\epsilon$ & Displacement height \\
$\lambda$ & Roughness density parameter, defined as $A_{F} / A_{S}$ \\
$\lambda_{M}$ & Roughness density parameter, defined as $k / L$ \\
$\lambda_{D}$ & Roughness density parameter, defined as $s / L$ \\
$\nu$ & Kinematic viscosity \\
$\Pi$ & Wake parameter \\
$\rho$ & Mass density of flow \\
$\sigma$ & Standard deviation \\
$\theta$ & Momentum thickness \\
\hline Subscript & Control volume surfaces (see Figure 3.1 ) \\
\hline $1,2,3,4,5$ & Subscripted quantity associated with air \\
$a i r$ & Subscripted quantity associated with water \\
$H_{2} O$ &
\end{tabular}




\section{Chapter 1}

\section{Introduction}

\subsection{Motivation}

The following thesis concerns the study of various rough walls and their impact on the structure of a two-dimensional turbulent boundary layer. Measurements of mean velocities, Reynolds stresses, and triple products allow for a detailed description of the flow over these rough walls. This study is unique in that it encompasses a relatively large variety of wall geometries, allowing for an examination of the relationships between the structure of the turbulent boundary layer and various parameters associated with the geometry of the rough wall.

This research is part of a larger effort (George and Simpson, 2000, 2004; Bennington, 2004) to understand the physics of the flow over rough walls and develop a deterministic model for rough wall turbulent boundary layers. The variety of cases studied in the present research contribute to the database necessary to fulfill this ultimate objective. The rough wall data presented here will also provide valuable test cases to evaluate turbulent models associated with boundary layer calculations. The remainder of this chapter serves as a brief review of previous research done that is relevant to the results presented in this thesis. 


\subsection{Rough Wall Turbulent Boundary Layers}

To at least as far back as Hagen (1854) and Darcy (1857), researchers have been interested in the effects of roughness on the structure of the boundary layer. An understanding of the mechanisms governing rough wall flow is essential to the analysis of a variety of situations, including ship hulls, pipes, and vegetation. Reviews by Raupach et al. (1991) and Jimenez (2004) provide an overview of the field.

Much focus has been placed on the study of the effects of two-dimensional roughness and mesh roughness, for example Perry et al. (1987) and Krogstad and Antonia (1999). However, many real-world situations are better described by distributed three-dimensional roughness elements, of which fewer studies have been done. A notable early study of three-dimensional roughness was that of Koloseus and Davidian (1966) which studied the flow over arrays of cubes.

In many roughness studies conducted, hot wire anemometry (HWA) is used to take measurements, and the resulting data sets are subject to the shortcomings of that method. HWA is inaccurate near a solid surface, so few studies have results near or below the height of the roughness element (Raupach et al., 1980; Perry et al., 1987). Studies that do contain data below the roughness height are largely measurements taken on actual or model vegetation, such as Raupach (1979).

George and Simpson (George and Simpson, 2000, 2004) conducted systematic studies of three-dimensional roughness elements, both isolated and distributed, in two- and three-dimensional boundary layers. This work was conducted using a fine-resolution three-dimensional Laser Doppler Velocimeter, allowing the measurement of all mean velocities, Reynolds stresses, and triple products throughout the boundary layer, including below the height of the roughness elements. 


\subsection{The Law of the Wall for Rough Walls}

Derived by asymptotic matching, it is well accepted that the law of the wall over a smooth surface is of the form

$$
\frac{U}{u_{\tau}}=\frac{1}{\kappa} \ln \frac{y u_{\tau}}{\nu}+C
$$

The effect of roughness on the law of the wall is to produce a shift $\Delta U / u_{\tau}$ of the velocity profile

$$
\frac{U}{u_{\tau}}=\frac{1}{\kappa} \ln \frac{y u_{\tau}}{\nu}+C-\frac{\Delta U}{u_{\tau}}
$$

This effect was first observed by Nikuradse (1933) in studies of sand roughened pipes. Prandtl and Schlichting (1934) proposed that the amount of the shift was related to the roughness Reynolds number, based on the sand grain height, $k_{s}$

$$
k^{+}=\frac{k_{s} u_{\tau}}{\nu}
$$

Prandtl and Schlichting (1934) defined three regimes based on the roughness Reynolds number and proposed limits on $k^{+}$for these regimes as follows; hydraulically smooth surfaces $\left(k^{+}<4\right)$ have no effect on the flow field, while over transitionally rough surfaces $\left(4<k^{+}<60\right)$ the roughness effect is dependent on viscosity. Finally, in fully rough flows $\left(k^{+}>60\right)$, the roughness effect is entirely due to the form drag on the elements and independent of viscosity. Thus, in this regime, the roughness effect $\Delta U / u_{\tau}$ is independent of Reynolds number and considered constant. The bounds on $k^{+}$are dependent on the type of roughness being studied, and subsequent authors have offered differing limits on the three regimes.

Schlichting (1936) measured the shift $\Delta U / u_{\tau}$ over surfaces roughened by cones, spheres, and other geometric shapes. These data clearly showed that the geometry and spacing of these elements had an effect on the size of this shift.

\subsection{Effects of Spacing and Geometry}

From a study of two-dimensional roughness, Morris (1955) proposed that a roughness element's effect on the boundary layer is primarily due to the 
element's downstream wake. Thus, the cumulative effect of a rough wall results from the interaction of the wakes of the various roughness elements. This concept led Morris to define three types of flow based on the roughness index $\lambda_{M}=k / L$, where $\mathrm{k}$ is the element height and $\mathrm{L}$ is longitudinal element spacing. For a wide spacing, the roughness elements are considered isolated, in which case the wake from one element has completely dissipated before the flow encounters the next. In interference flow, the elements are close enough such that the flow conditions at any element are affected by the wake and vorticity of the upstream element(s). Over the entire rough surface, flow near the wall is characterized by high turbulent mixing and vorticity. For a closer spacing of elements, a skimming flow develops, in which isolated, stable vortices develop between the roughness, and the main flow essentially "skims" the crests of the elements.

Subsequent authors built upon and refined the concept of a using a spacing or geometry parameter (usually denoted as " $\lambda$ ") to characterize a rough wall flow. This parameter is then correlated empirically to a function $f(\lambda)$ to predict the roughness effect. Betterman (1966) produced a correlation based on $\lambda_{M}$ as defined by Morris. The experimental data used by Betterman was limited to two-dimensional square bars. Dvorak (1969) defined a slightly different $\lambda_{D}=s / L$, where $s$ is the streamwise dimension of the roughness element. Dvorak used a slightly broader range of experimental data, and produced two correlations, for dense and sparse roughness densities. Simpson (1973) used an expanded data set and defined $\lambda$ as

$$
\lambda=\frac{A_{F}}{A_{S}}
$$

where $A_{F}$ is the frontal area of the elements normal to the flow and $A_{S}$ is the total wall surface area. Note that in the case of two-dimensional elements, Simpson's $\lambda$ is equivalent to that of Morris (1955) and Betterman (1966).

Wooding et al. (1973) developed a drag law for arrays of roughness elements, utilizing a large and varied group of experimental data sets. No flow dependent variables are used, so the drag is expressed as strictly a function of roughness spacing and geometry, and boundary layer thickness. An element "shape factor", defined as the ratio of element height to thickness, is incorporated, which is a more detailed description of the individual element geometry than is used in the above mentioned studies. The drag law is valid strictly for 
three-dimensional roughness elements, and the authors noted that the flow over two-dimensional bars is a fundamentally different case. Dirling (1973) and Waigh and Kind (1998) incorporated "bluntness," defined as the ratio of upstream-facing wetted area to upstream-facing frontal area, to characterize the element geometry in their correlations of the roughness effect.

George (2005) evaluated various roughness density correlations, and concluded that the correlation of Dvorak (1969) using $\lambda$ as defined by Simpson (1973), produced the best agreement with experimental results, despite the added detail of later correlations.

\subsection{Roughness and the Law of the Wake}

Coles (1956) developed the Law of the Wake, which expressed the velocity profile in the outer region of the boundary layer as a function added to the logarithmic overlap region,

$$
\frac{U}{u_{\tau}}=\frac{1}{\kappa} \ln \left(\frac{y u_{\tau}}{\nu}\right)+C+\frac{\Pi}{\kappa} W\left(\frac{y}{\delta}\right)
$$

The wake parameter $\Pi$ has a smooth wall value of approximately 0.52 (Fernholz and Finley, 1996) for zero pressure gradient boundary layers. Typically, the rough wall value for $\Pi$ is similar to the smooth wall value (as in Hama, 1955). Some literature has indicated widely varied values for $\Pi$, however. In a review, Tani (1987) analyzed several data sets and obtained values of $\Pi$ ranging from 0 to 1 for $k$-type roughness, and a smaller range (0.6-0.8) for $d$-type surfaces.

Jimenez (2004) attributes the large range of values associated with $k$-type roughness obtained by Tani to the relatively large ratio of $k / \delta$. When the height of the roughness elements is small compared with the boundary layer thickness, the roughness has little direct effect on the flow away from the wall. However, as noted by Simpson (1973), if $k / \delta$ is large, the vortices produced by the roughness elements may have an effect throughout the boundary layer. A large $k / \delta$ ratio produces a weak logarithmic layer, making the definition of $\Pi$ difficult (Jimenez, 2004). 


\subsection{Displacement Height}

There is some ambiguity in the definition of the origin of the vertical coordinate $y$, since the presence of roughness elements displaces the flow streamlines away from the wall. This effect is accounted for by placing the origin a distance $\epsilon$ from the wall, referred to as the displacement height. Many authors have developed schemes for estimating or calculating this quantity.

Some, such as Schlichting (1936), took the displacement height to be merely the thickness added to the wall if the volume of the roughness elements was evenly distributed to form a smooth wall. This method is inadequate in that it does not account for the configuration of the roughness elements nor their actual effect on the flow. As noted by Coleman et al. (1984), Schlichting's displacement height, though logical, is unrelated to any characteristic of the rough wall velocity profile.

Thom (1971) proposed that the displacement height is the location at which the mean drag force appears to act. This was later verified by Jackson (1981), who also showed that displacement height corresponded to the height at which the wall shear stress appears to act. Determination of the displacement height could be accomplished through the calculation of the centroid of the drag profile.

\subsection{Transitional Roughness}

Koloseus et al. (1962) explained that even for roughness elements that are large in height compared to the viscous sublayer, the flow may still be transitionally rough when the elements are spaced far enough apart. If the smooth surface is sufficiently exposed, viscous effects become significant in addition to the form drag on the elements. The results of Chen and Roberson (1974) provide an example of this; in an experiment involving distributed hemispherical elements, flows with a Reynolds number of $k^{+}<200$ were still in the transitional regime. This is well above the previously cited limits of Prandtl and Schlichting (1934), due to the fact that those experiments were performed over closely packed sand grains, in which the flow regime is dependent solely on element height. 
Typically, researchers have avoided transitionally rough flows, due to the fact that is difficult to analyze the dependence of $\Delta U / u_{\tau}$ on Reynolds number. Colebrook (1939) collected data on transitionally rough industrial pipes and produced a correlation between $\Delta U / u_{\tau}$ and the fully rough equivalent sand roughness Reynolds number. This correlation was subsequently used to generate the Moody chart (Moody, 1944) for skin friction. However, as noted by Bradshaw (2000), this industrial roughness is polydisperse and therefore

produces different results than the more regular roughness commonly studied in laboratory experiments.

\subsection{Overview of Thesis}

Chapter two of this thesis describes the experimental apparatus and setup, including the wind tunnel, LDV system, test articles, and data processing. Chapter three provides a description of the results, including the presentation and detailed discussion of the mean velocities, Reynolds stresses, triple products, and other quantities derived from these data. Chapter four contains an overview of the flow around the roughness elements, based upon the observations of the previous chapter, and discusses trends in mean velocity profile parameters as functions of roughness geometry. In the fifth chapter, a summary and conclusions will be presented, closing with recommendations for future research.

Several appendices follow the main body of the thesis. Appendix A will present a complete set of plots of all data, as some plots are not included in the main body of the thesis. Appendix B provides an analysis of the uncertainties of measured and derived quantities. Appendix C provides the details of a wind tunnel seeding study conducted separately from this research. 


\section{Chapter 2}

\section{Apparatus and Instrumentation}

\subsection{Small Boundary Layer Wind Tunnel}

All experiments were conducted in the Virginia Tech Small Boundary Layer Wind Tunnel, shown in Figures 2.1 and 2.2. Bennington (2004) has a detailed description of the tunnel and its various components.

\subsubsection{Tunnel Overview}

The wind tunnel is a closed return wind tunnel which is driven by a 7.5 horsepower centrifugal blower. Flow variations introduced by the blower are damped by a plenum chamber which contains a baffle plate. After exiting the plenum, the flow enters the first of two contractions which reduces in cross section from $53 \times 53 \mathrm{~cm}$ to $38 \times 23 \mathrm{~cm}$ over a distance of $46 \mathrm{~cm}$. This contraction leads to a honeycomb section and then a series of three screens, reducing the turbulence level in the flow. The second contraction reduces from $41 \times 24 \mathrm{~cm}$ to $10 \times 24 \mathrm{~cm}$.

The flow passes from the second contraction into the test section, which approximately $200 \mathrm{~cm}$ long and $24 \mathrm{~cm}$ wide. The height of the plexiglass ceiling is adjustable through the use of five support struts distributed along the length of the test section. These adjustments allow for the maintenance of a zero streamwise pressure gradient. The ceiling has twenty-four holes with 


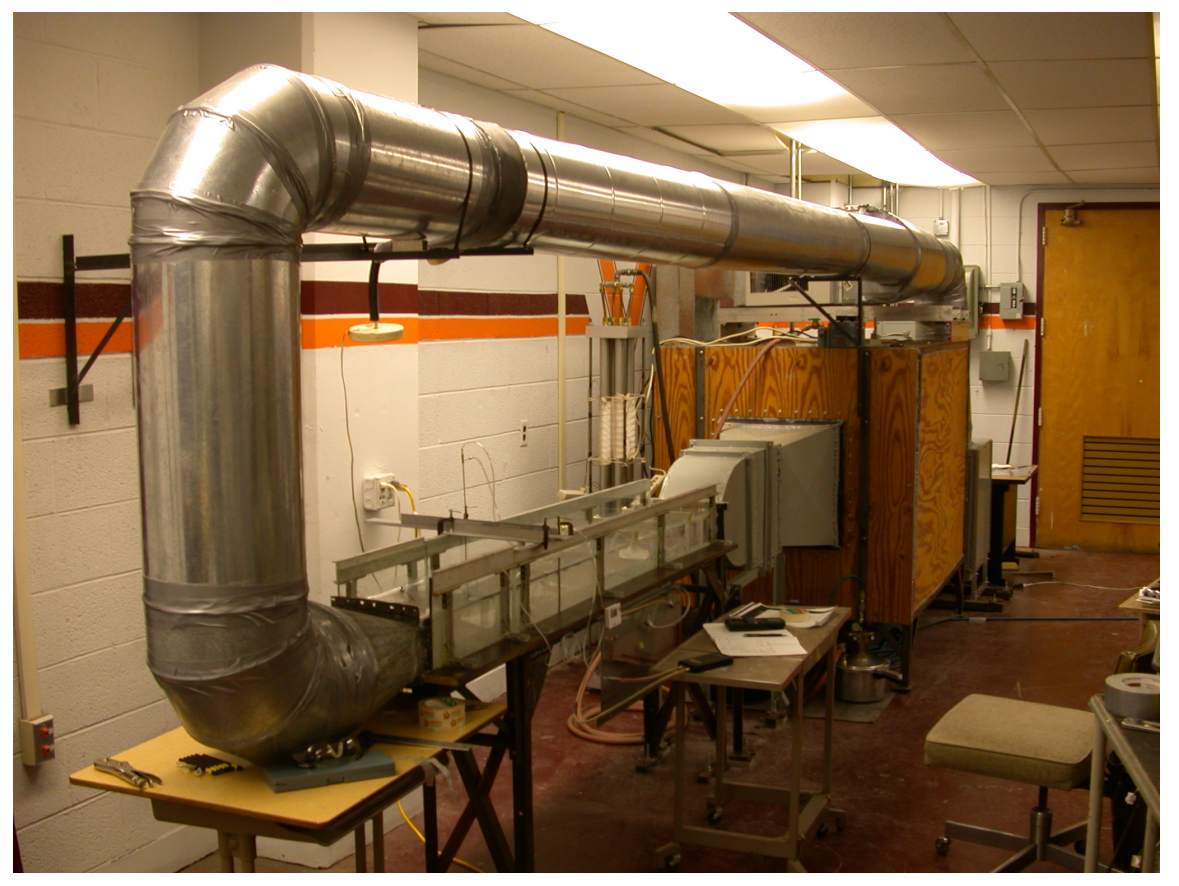

Figure 2.1: Virginia Tech Small Boundary Layer Wind Tunnel 


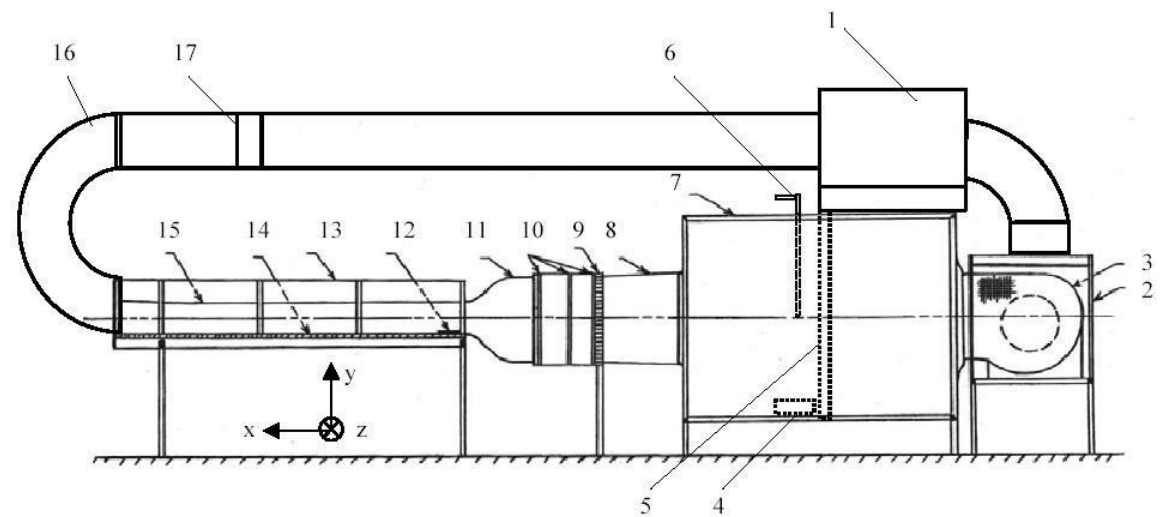
1. Air Conditioner
2. Filter Box
3. Blower
4. Drip Pan
5. Baffle Plate
6. Seeding Pipe
7. Plenum Chamber
8. Contraction
9. Honeycomb

10. Screens

11. Contraction

12. Trip Arrangement

13. Glass Side Walls

14. Bottom Wall

15. Top Wall

16. Return Ducting

17. Rubber Joint

Figure 2.2: Schematic of wind tunnel. Ref. Bennington (2004) 
plugs distributed along its centerline. These holes allow for probe access in order to measure the pressure gradient and free-stream velocity.

At the beginning of the test section is a trip arrangement designed to force transition to turbulent flow and artificially thicken the boundary layer. This arrangement consists of an initial $0.32 \mathrm{~cm}$ square bar, followed by a second $0.32 \mathrm{~cm}$ square bar located $5.40 \mathrm{~cm}$ downstream of the first. The floor of the test section for a length of $40 \mathrm{~cm}$ downstream of the second bar, as well as the area between the two bars, is covered in 20 grit silicon carbide sandpaper.

An optical float glass disc which is inset in the floor of the test section provides access for LDV measurements. The center of this disc is located at a distance of $116.7 \mathrm{~cm}$ from the beginning of the test section.

After a short diffuser section at the back of the tunnel, the flow enters the $77.5 \mathrm{~cm}$ diameter return ducting. As seen in Figures 2.1 and 2.2, the tunnel is in a vertical return configuration due to space considerations. An air conditioner maintains the test section temperature at $25^{\circ} \mathrm{C} \pm 1^{\circ} \mathrm{C}$. The flow passes through a butterfly valve used to control the flow speed and a filter box which removes any debris before entering the blower.

\subsubsection{Flow Conditions}

For the purposes of these experiments, tunnel conditions were nominally a zero pressure gradient with a $27.5 \mathrm{~m} / \mathrm{s}$ free-stream velocity at $25^{\circ} \mathrm{C}$. As mentioned previously, there are 24 ports along the length of the wind tunnel ceiling through which dynamic pressure is measured in order to set the free-stream velocity and verify that there is a zero pressure gradient. Measurements were taken with a Pitot-static probe and a liquid manometer to ensure that at each port, the free-stream velocity $U_{\infty}=27.5 \mathrm{~m} / \mathrm{s}$. Measurements were taken every $13-15 \mathrm{~cm}$ at the ports along the length of the test section ceiling, and the free-stream velocity was set within the measurement uncertainty of \pm 0.02 inches. This results in an uncertainty in the freestream velocity of approximately $\pm 0.1 \mathrm{~m} / \mathrm{s}$. Temperature is monitored by a digital thermometer inside the test section.

Data taken by Bennington (2004) in the Small Boundary Layer Wind 


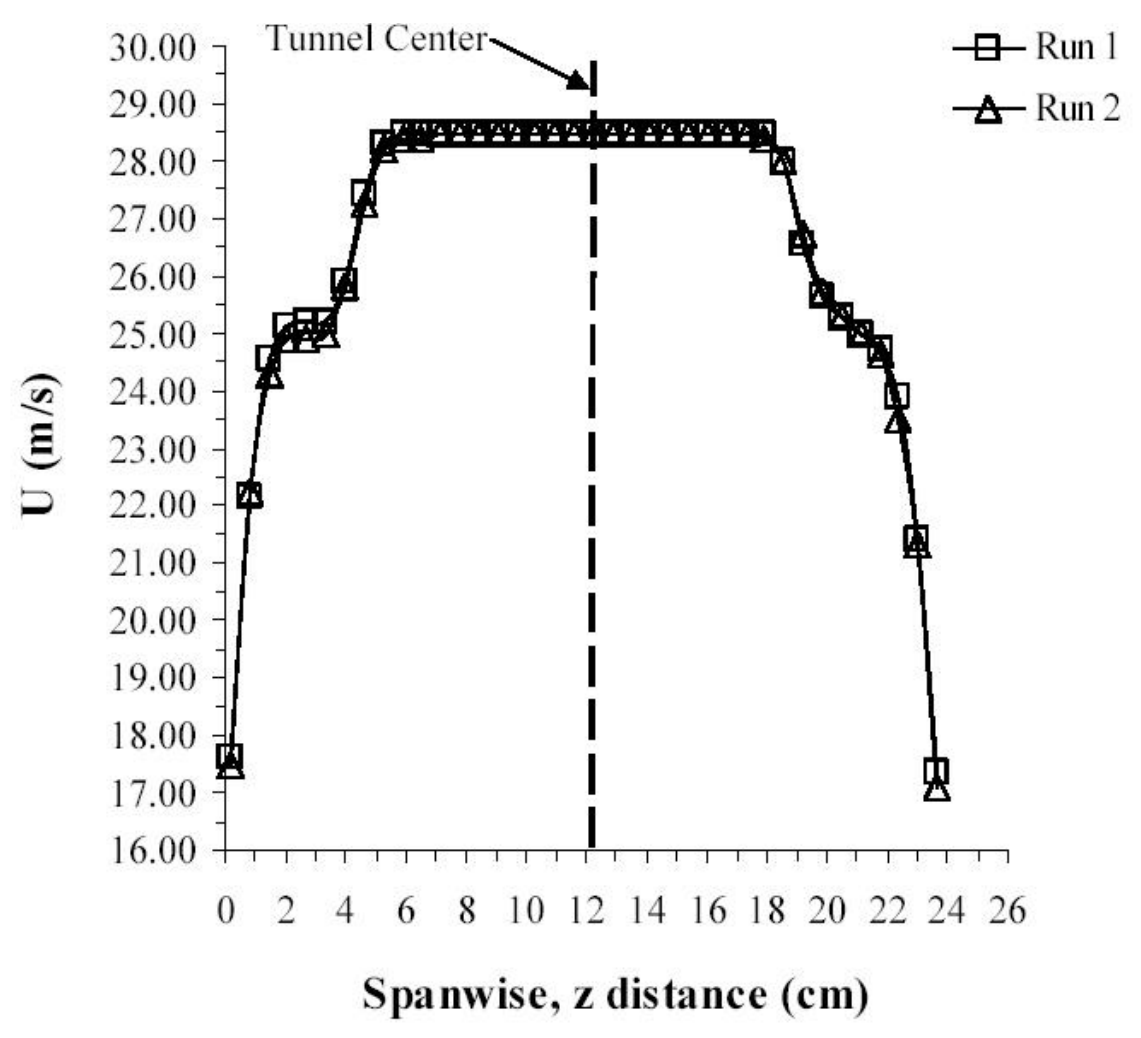

Figure 2.3: Spanwise traverse of wind tunnel test section at mid-height. Ref. Bennington (2004)

Tunnel shows the region of constant velocity in the test section. Figure 2.3 shows the results of a spanwise Pitot-static probe traverse at the midheight of the test section. The data of Bennington were taken in the same streamwise region where data was taken for this study. A constant velocity region of approximately $12 \mathrm{~cm}$ wide is visible (note that in these data the free-stream was not set at the typical value of $27.5 \mathrm{~m} / \mathrm{s}$ ). All roughness measurements taken in the present research occur well within this region so as not to be affected by the test section walls and associated boundary layers.

Figure 2.4 shows several smooth wall LDV profiles taken in the Small Boundary Layer Wind Tunnel, compared with direct numerical simulations (DNS) of Spalart (1988). The discrepancies between the DNS and experimental data away from the wall are due to widely differing Reynolds num- 
bers. These LDV profiles were taken in the center of the tunnel, $116.7 \mathrm{~cm}$ downstream of the contraction, as well as $2.26 \mathrm{~cm}$ to either side of the tunnel centerline. The similarity of these profiles (within 5\%) show the twodimensionality of the flow in the region where data is taken.

\subsubsection{Seeding System}

The seed material for the LDV measurements is di-octyl phthalate (DOP), which is atomized by a Laskin nozzle aerosol generator. The mean particle size is $0.7 \mu \mathrm{m}$, with a geometric standard deviation of about $22 \%$, as measured by a TSI Aerodynamic Particle Sizer. The aerosol generator is operated at approximately 11 psi, which provides a sufficient data rate. The seed material leaves the generator and travels through a hose to where it is injected into the plenum chamber of the wind tunnel (see Figure 2.2, item \#6).

\subsection{LDV System}

With a few exceptions to be discussed in a later section, all measurements in this research were taken with a three-component fiber optic laser doppler velocimeter (LDV). The system consists of an optical table upon which the measurement laser beams are generated from the source laser, a probe head which focuses the beams into a measurement volume and collects the scattered light. Photomultiplier (PM) tubes convert this collected light to an electrical signal, which is then amplified and conditioned. Data acquisition is accomplished by a PC-based system which makes use of Frequency Domain Processors as analog triggering devices.

\subsubsection{Optical Table}

The optical table and associated components are shown in Figures 2.5 and 2.6. The laser used in this system is a Coherent Innova-90 argon-ion laser. The emitted beam is divided into its constituent colors by a set of two prisms. A polarization rotator located before the prisms optimizes the light polarization so as to lose as little spurious light as possible from the prisms. Only 

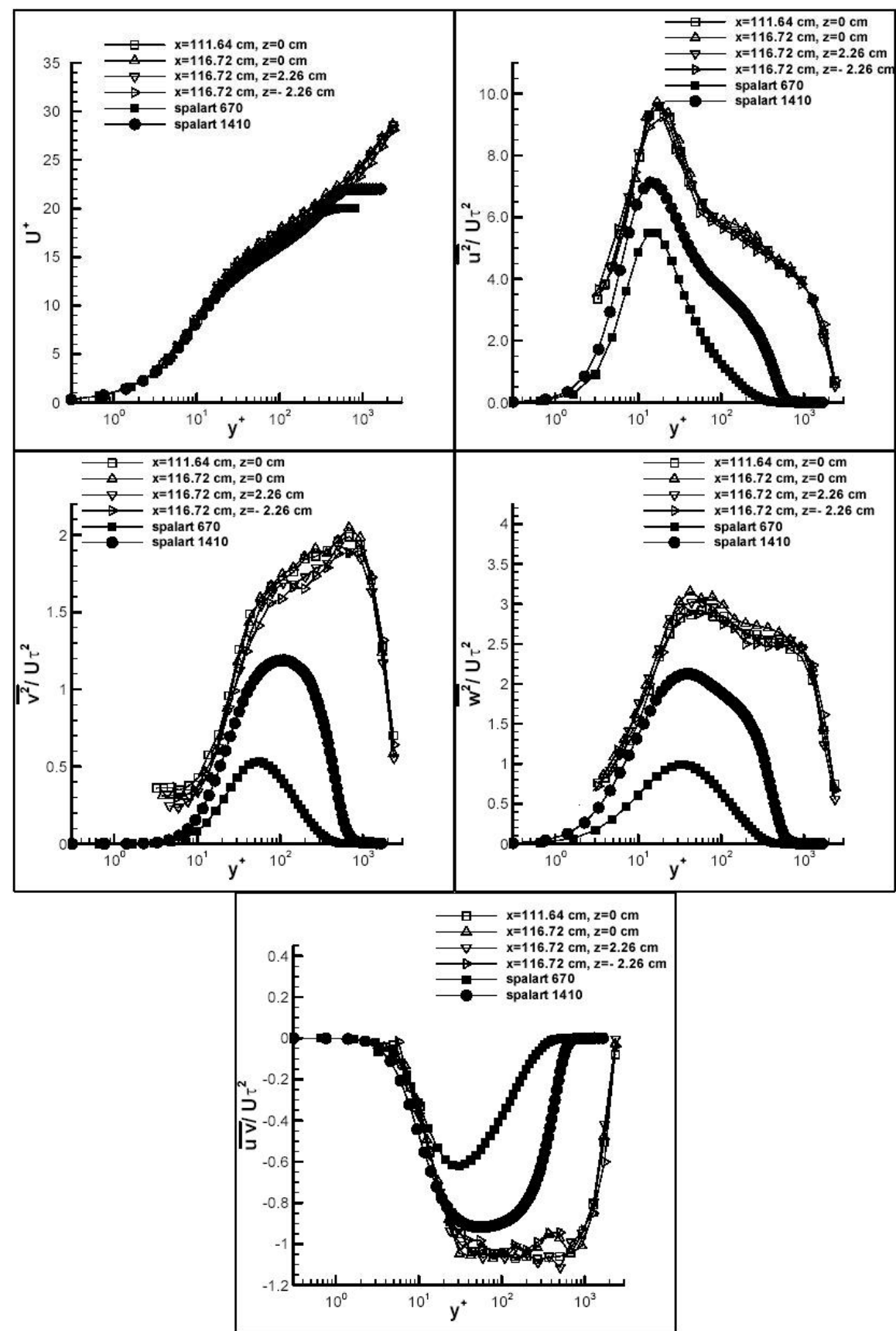

Figure 2.4: Smooth Wall LDV profiles, along with Spalart (1988) DNS data 
the two strongest color beams, the $514.5 \mathrm{~nm}$ green and $488 \mathrm{~nm}$ blue beams, are utilized. The green beam passes through a polarization rotator and a beam splitter cube, producing two beams. One green beam passes through a $50 \mathrm{MHz}$ Bragg cell, and the other through a $27 \mathrm{MHz}$ Bragg cell. Of the four resulting beams (two unshifted beams, one $27 \mathrm{MHz}$ shifted beam, and one $50 \mathrm{MHz}$ shifted beam), one unshifted beam is not utilized. The other three beams are launched into polarization-preserving, single-mode fiber optic cables by fiber couplers. The blue beam goes through a $40 \mathrm{MHz}$ Bragg cell and both resulting beams are launched into fiber optic cables. For an explanation of the use of frequency shifted beams to eliminate velocity ambiguity, see Absil (1995). The fiber optic cables transmit the laser beams to the LDV probe head.

\subsubsection{Probe Head}

The LDV probe head is shown in Figures 2.7 and 2.8. The probe head consists of two transmitting assemblies, oriented at 90 degrees to each other, and a central receiving assembly. The three green beams emit from one transmitting assembly and are crossed via transmitting lenses to form two sets of fringes. The third set of fringes is formed by the blue beams from the second transmitting assembly. These five beams, and associated three measurement volumes, are aligned using the adjustments on the probe head. For a description of alignment technique, see Chesnakas (1995) and Tang (2004). This measurement volume is calculated to be of approximately $50 \mu \mathrm{m}$ in diameter with fringe spacing of $5 \mu \mathrm{m}$, based on calculations presented in Durst et al. (1981) and Durst et al. (1995). This focusing point, or measurement volume, also coincides with the focus of the receiving optics, which collects the side-scattered laser light and focuses onto a multi-mode fiber optic cable.

\subsubsection{Signal Conditioning \& Data Acquisition System}

The multi-mode fiber optic cable transmits the collected light to the photomultiplier (PM) tubes. A dichroic filter separates the blue and green light signals, which then pass to two Electron Tubes Limited PM tubes, models 9124B and 9125B, respectively. Via the photoelectric effect, the photocath- 


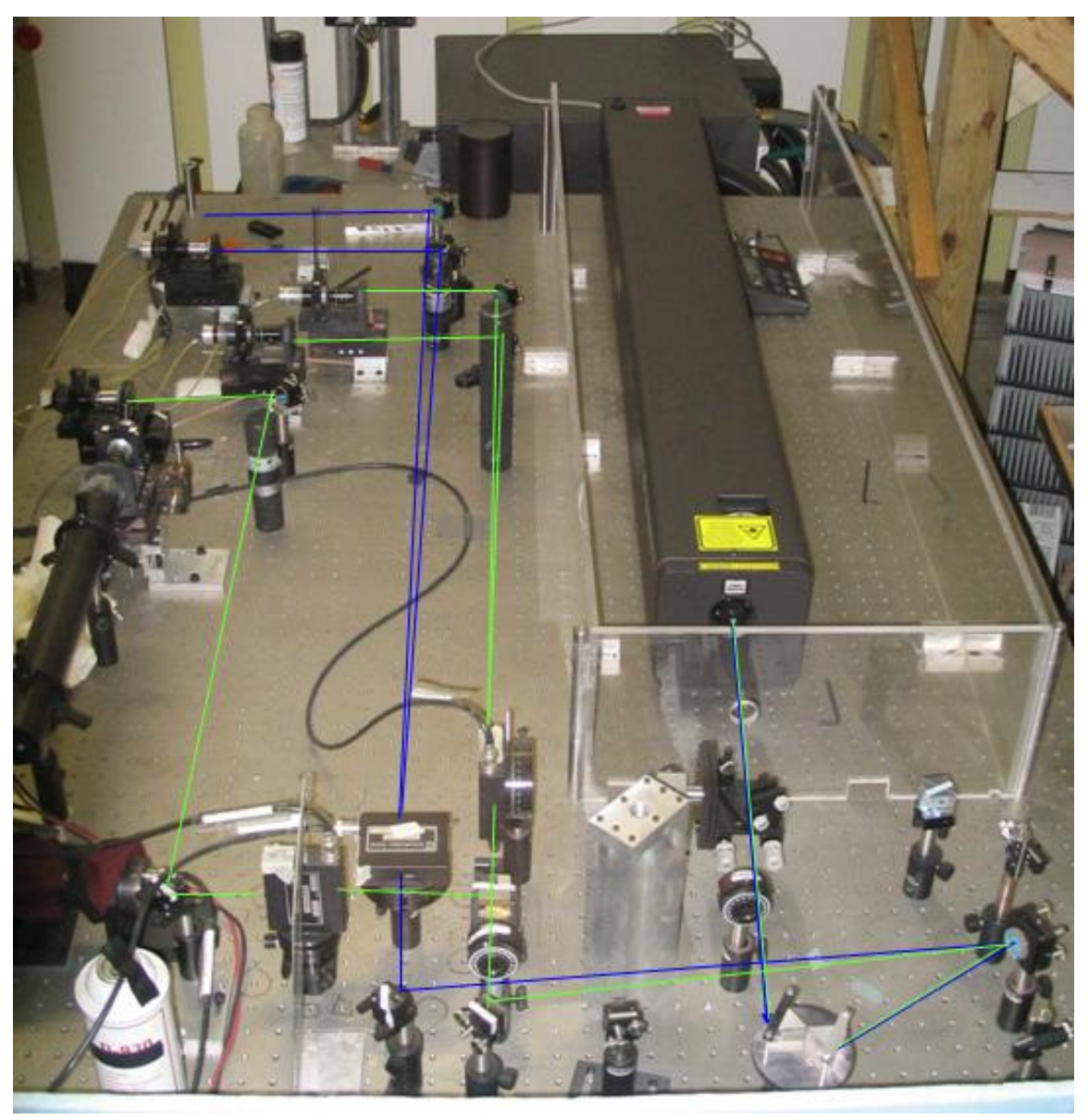

Figure 2.5: Photograph of the LDV optical table 


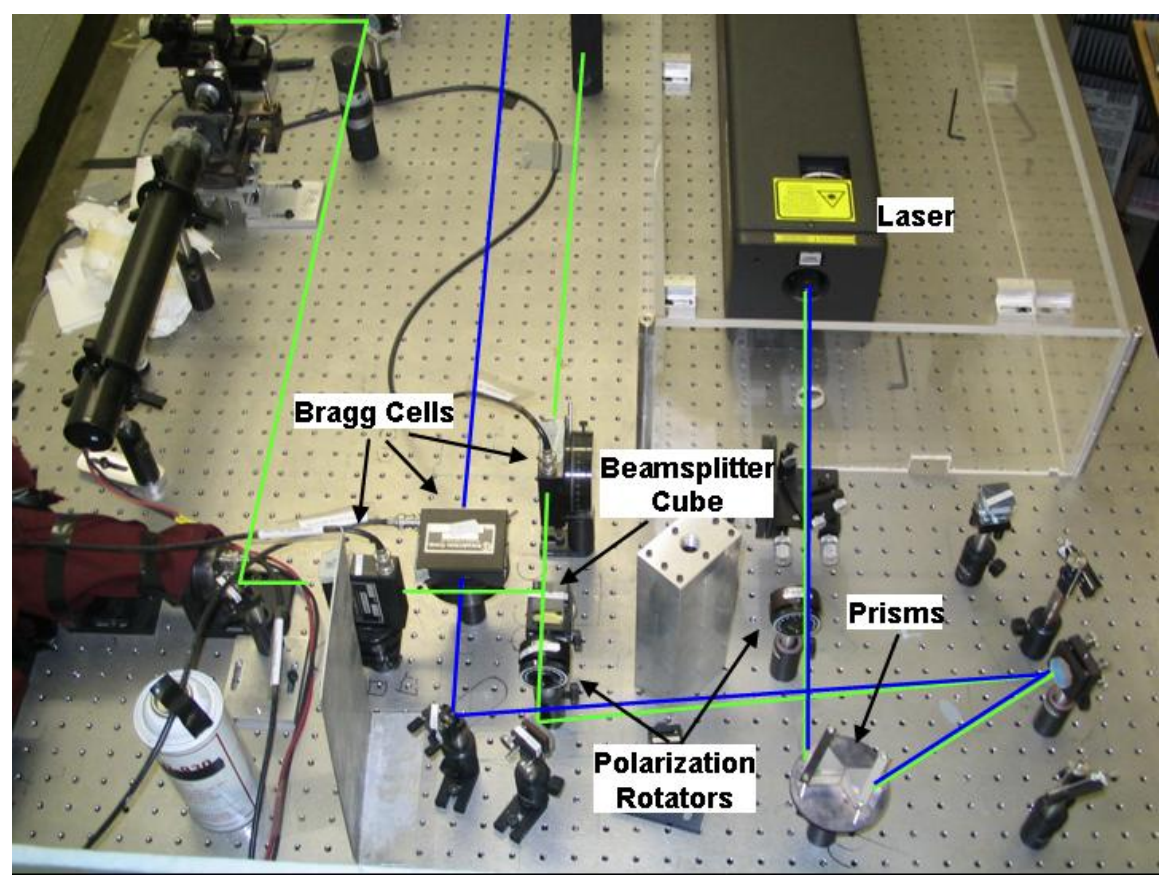

Figure 2.6: Optical table components at exit of laser

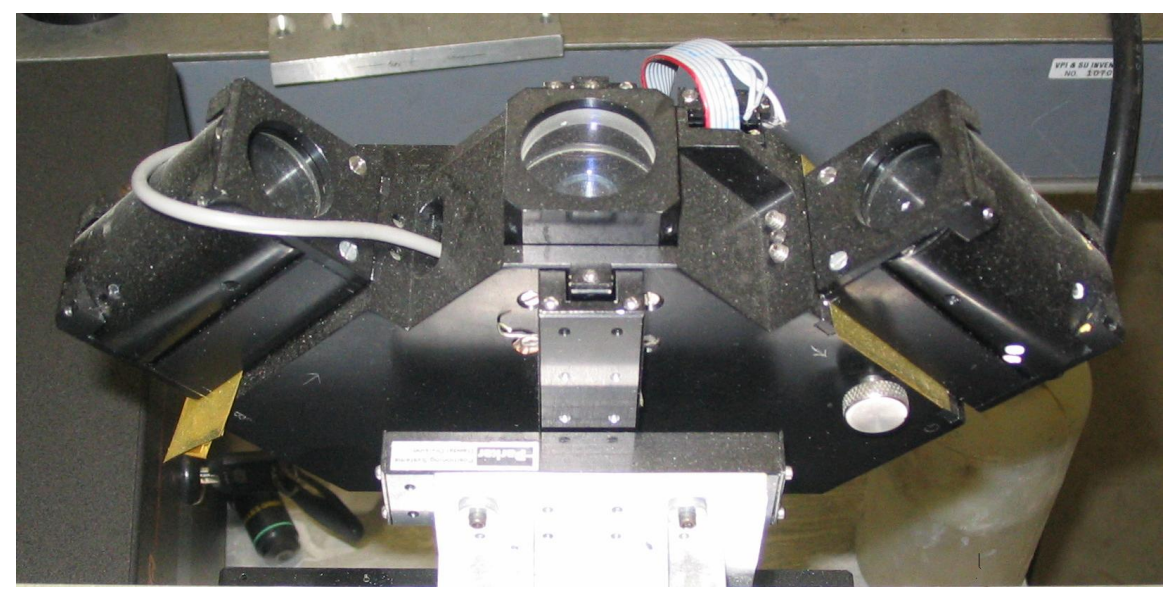

Figure 2.7: Photograph of LDV probe head 


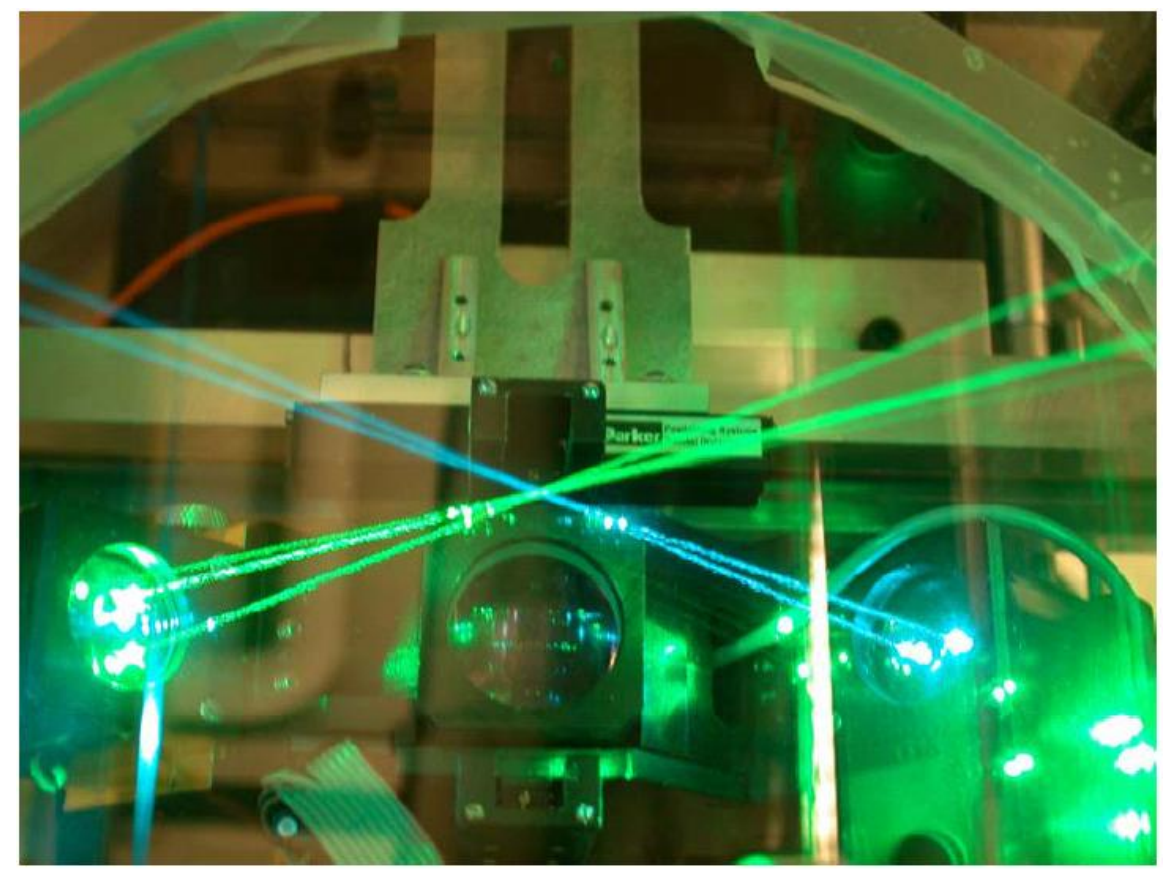

Figure 2.8: LDV probe head while taking data 
ode which forms the face of the PM tube emits electrons in proportion to the collected photons. This resulting current is amplified by a dynode chain, also contained within the photomultiplier unit. The signal produced by the PM tube must be conditioned before data can be acquired.

The green signal is split, so that the signals corresponding to the 50 $\mathrm{MHz}$ and $27 \mathrm{MHz}$ Bragg-shifted beams can be processed separately. These two signals, along with the signal resulting from the blue PM tube, are each amplified by two Mini-Circuits amplifiers. Signals from three radio frequency $(\mathrm{RF})$ generators are similarly amplified, and mixed with the three data signals in order to place the resulting signal within the measurement bandwidth. The RF generator settings are recorded at each data point for later use in the data reduction. Three $7.75 \mathrm{MHz}$ low-pass filters are used to eliminate extraneous signal above the measurement bandwidth.

The three resulting, downmixed signals are sent to three Macrodyne FDP3100 frequency domain processors (FDPs) which are used solely as analog triggering devices. The coincidence window for a validated trigger is set at $10 \mu \mathrm{s}$. The trigger levels are maintained such that the amount of peak-to-peak validated triggers on each Macrodyne is $97 \%$ or greater.

The trigger signal is used in the operation of the PC-based data acquisition system, which was developed by Lowe (2005). The trigger signal from the Macrodynes, along with the three signals corresponding to the three beam pairs are connected to two National Instruments NI PCI-5112 data acquisition cards contained in the data acquisition PC. Each NI PCI-5112 can sample 2 channels of data at $100 \mathrm{MS} / \mathrm{s}$ with 8 bit resolution and 16 MB of deep memory. The data acquisition software is written in LabView, which easily integrates with the data acqusition cards. Six $\mu s$ of pre- and post-trigger data are acquired, and a fast Fourier transform is performed on the center ten $\mu s$. After validating the samples, the three measured frequencies, along with the sample number and arrival time, are saved to a data file. For each measurement location, 15,000 to 30,000 samples are acquired, depending on the proximity to the tunnel floor and data rate. 


\subsubsection{Data Processing}

After data acquisition is complete, noise (mostly resulting from electronic components) is eliminated, the velocity data in the probe co-ordinate system are rotated to align with the wind tunnel coordinate system, and turbulence quantities are calculated.

The noise reduction method is similar to that described in Olçmen and Simpson (1995). At each data point, there are three histograms corresponding to the three measured velocity components. The histograms are plotted on a semilogarithmic vertical scale. A parabola is fit to the upper half of the histogram (greater than the mean) from 80 percent of the histogram's peak to its base. This procedure is done simultaneously for the lower half of the histogram. Samples which fall outside of this parabolic "skirt" are eliminated. A sample located outside this skirt on even one of its velocity components is discounted entirely.

After this initial noise reduction, the data is rotated from the three measured velocity components (parallel to the cross-products of the crossed laser beam pairs) to the wind tunnel coordinate system. This process is accomplished through the use of a rotation matrix determined by the manual measurement of the laser beam angles.

Following this coordinate rotation, noise reduction is repeated using the above described method. From these "clean" data, mean velocities, Reynolds stresses, and triple products are calculated.

Because the LDV probe is not perfectly aligned with the tunnel coordinate system, a final rotation is done. These rotation angles are determined by rotating the data to find minimum $\bar{w}$ and $v \bar{w}$ values on smooth wall, twodimensional boundary layer profile data. After determining these rotation values, the probe is not removed from its stand, so the same angles are used for the rough wall data. These angles are less than $2^{\circ}$. 


\subsection{Outer layer LDV System}

The three-component LDV system described above is designed for near-wall measurements, which was the region of interest in the present research. Thus, this system was generally adequate for the required measurements. However, the three-component system could only measure to a distance of approximately $29.6 \mathrm{~mm}$ from the wall, which did not reach the free-stream. To complete the mean streamwise velocity profile in the outer layer, a one-component Laser Doppler Velocimeter was utilized.

This system, pictured in Figure 2.9, is part of a larger, "Comprehensive" LDV system, whose details are beyond the scope of this document. For a description, see Lowe (2005). It is sufficient for purposes here to state that redundant measurements are taken of the same velocity component by four beams, which form two overlapping measurement volumes. Using the same PM tubes, signal conditioning, and data acquisition hardware described above (in a two- rather than three-component configuration), 1,000-2,000 samples are taken of the streamwise velocity, from which the mean velocity is calculated. About 10,000 samples are taken at the outermost point, in the free-stream, for calibration purposes. Typically, only 3-4 outer points, located beyond the data taken by the three-component system, are used to complete the velocity profile.

By virtue of being a one-component system, the Outer Layer LDV is subject to biases resulting from high turbulence levels or velocity gradients. For this reason, data was only taken with this system in the outermost region of the boundary layer, where there are comparatively lower levels of turbulence and small velocity gradients. The use of this data was limited in the following analysis, and is noted where appropriate.

\subsection{Traverse and Location of Wall}

Both the three-component and outer layer LDV systems are mounted on the same traverse system, which was designed by Lowe (2005). The traverse is capable of motion along three axes, which are aligned parallel to the tunnel coordinates. Motion in all three axes is motor-driven, and position can be 


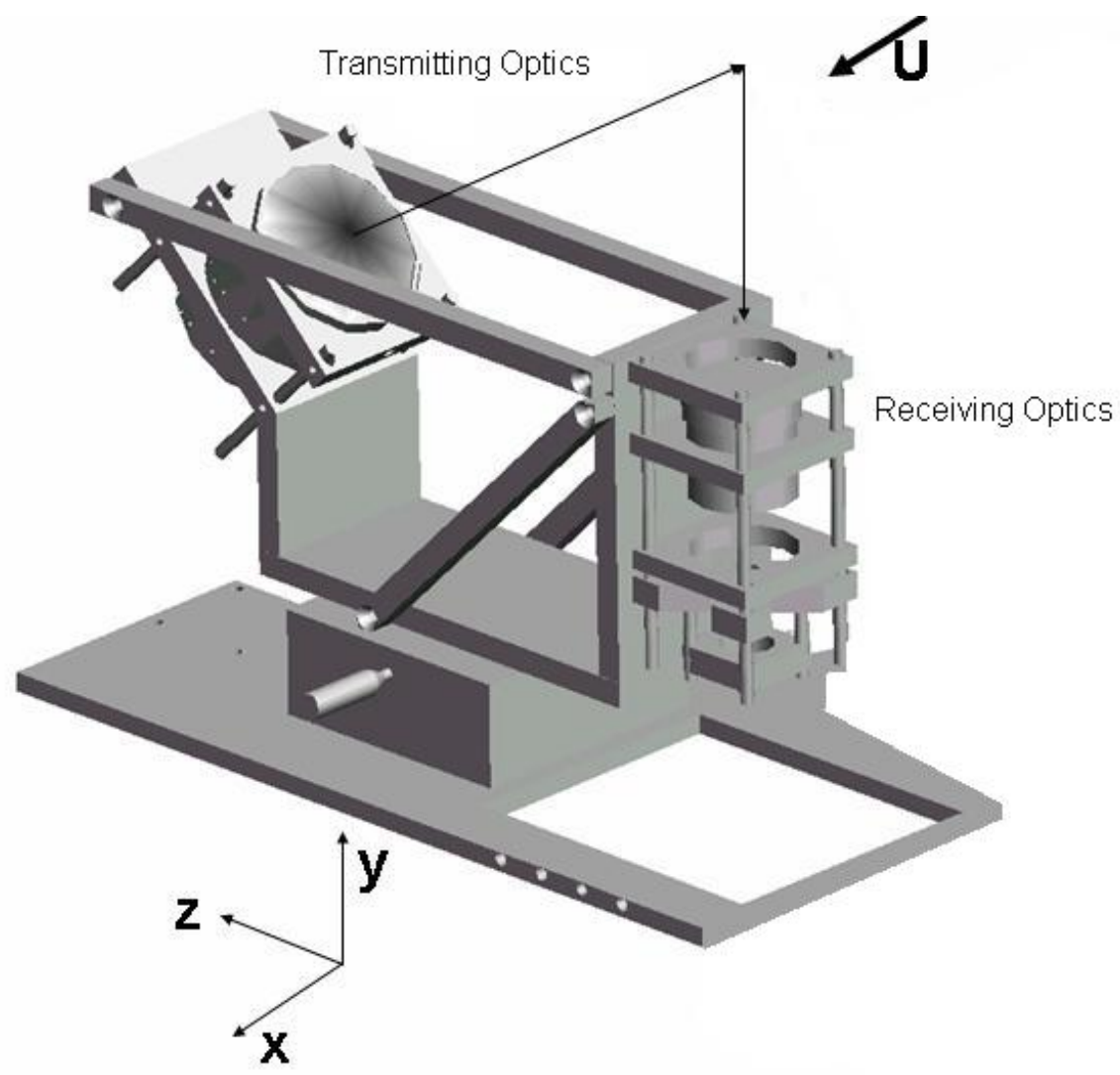

Figure 2.9: Outer Layer LDV System. Figure adapted from Lowe (2005). 


\begin{tabular}{|c||c|c|c|c|c|}
\hline Case \# & Element Shape & Orientation & Spacing $(\mathrm{mm})$ & Height $(\mathrm{mm})$ & $\lambda^{-1}$ \\
\hline \hline 1 & Gaussian & Straight & 5.49 & 2.54 & 11.3 \\
\hline 2 & Gaussian & Staggered & 5.49 & 2.54 & 11.3 \\
\hline 3 & Gaussian & Straight & 8.23 & 2.54 & 25.5 \\
\hline 4 & Gaussian & Staggered & 8.23 & 2.54 & 25.5 \\
\hline \hline 5 & Cylinder & Straight & 10.97 & 0.38 & 160 \\
\hline 6 & Cylinder & Staggered & 10.97 & 0.38 & 160 \\
\hline 7 & Cylinder & Straight & 10.97 & 0.76 & 80 \\
\hline 8 & Cylinder & Staggered & 10.97 & 0.76 & 80 \\
\hline 9 & Cylinder & Straight & 10.97 & 1.52 & 40 \\
\hline 10 & Cylinder & Staggered & 10.97 & 1.52 & 40 \\
\hline
\end{tabular}

Table 2.1: Roughness fetch configurations

controlled either by a remote control or a LabView PC-based controller communicating via a serial port. Resolution in the two horizontal directions is approximately $5 \mu \mathrm{m}$, while vertically, position can be determined to within $1 \mu \mathrm{m}$ due to the presence of a linear encoder with a digital readout.

\subsection{Roughness Fetches}

Ten different rough wall configurations are discussed in this thesis, which are summarized in Table 2.1. Included in Table 2.1 is the inverse of $\lambda$, a parameter combining various length scales as defined by Simpson (1973). The parameter is the ratio of the element front area normal to the flow to the total wall surface area (see Equation 1.4). For example, for a cylindrical post element

$$
\lambda^{-1}=\frac{D^{2}}{k \cdot d}
$$

where $D$ is the the spacing between elements, $k$ is the element height, and $d$ is the element diameter. The data for the first case were taken by Bennington (2004) and described briefly in an appendix therein. The data for the nine remaining cases were taken by this author. 


\subsubsection{Description}

The element shapes examined are Gaussian spikes and circular cylindrical posts. The gaussian spikes have a height of $2.54 \mathrm{~mm}$ and a base diameter of $2.54 \mathrm{~mm}$. The profile of the spike is defined by the equation

$$
y=2.54 e^{-20.27 z^{2}}
$$

where $y$ is the height and $z$ is the element radius. Close up photographs of the roughness fetches can be seen in Figure 2.10. The cylindrical posts all have base diameters of $1.98 \mathrm{~mm}$, but three different heights are studied: 0.38 , 0.76 , and $1.52 \mathrm{~mm}$.

Two different spacings of Gaussian spike elements are considered. The first is $5.49 \mathrm{~mm}$ between elements $(D / d=2.16)$, the second is a larger spacing of $8.23 \mathrm{~mm}(D / d=3.24)$. These fetches were installed in the wind tunnel in two different arrangements, referred to as "straight" and "staggered." In the straight case, the rows and columns of elements are oriented parallel and perpendicular to the free-stream flow direction, while in the staggered case, the rows and columns are oriented at $45^{\circ}$ to the flow.

All fetches of cylindrical elements studied have a spacing of $10.97 \mathrm{~mm}$ $(D / d=5.54)$. Each of the three heights is studied in both the straight and staggered configurations.

The roughness fetches cover a length of the tunnel floor of approximately

$95 \mathrm{~cm}$, from the back of the sand paper mentioned in Section 2.1.1 to about $25 \mathrm{~cm}$ downstream of the measurement location. Figure 2.11 shows roughness installed on the wind tunnel floor. The tunnel floor is covered in roughness fetch with carbon fiber substrate (details will be included in section 2.5.2), except for the area of the floor occupied by the optical glass disc through which measurements are made. In the case of the cylindrical elements, the fetch has a cellophane tape substrate, and in the case of the Gaussian elements, there is a gauze substrate. A thin slot of gauze or cellophone is cut away to allow optical access for the LDV measurements (see Figure 2.12). These substrates are much thinner than the carbon fiber, so there less of a "step" from the fetch to the surface of the glass to disrupt the near-wall flow.

For each roughness fetch configuration, a total of nine vertical LDV profiles are acquired. Example measurement locations are included in Figures 

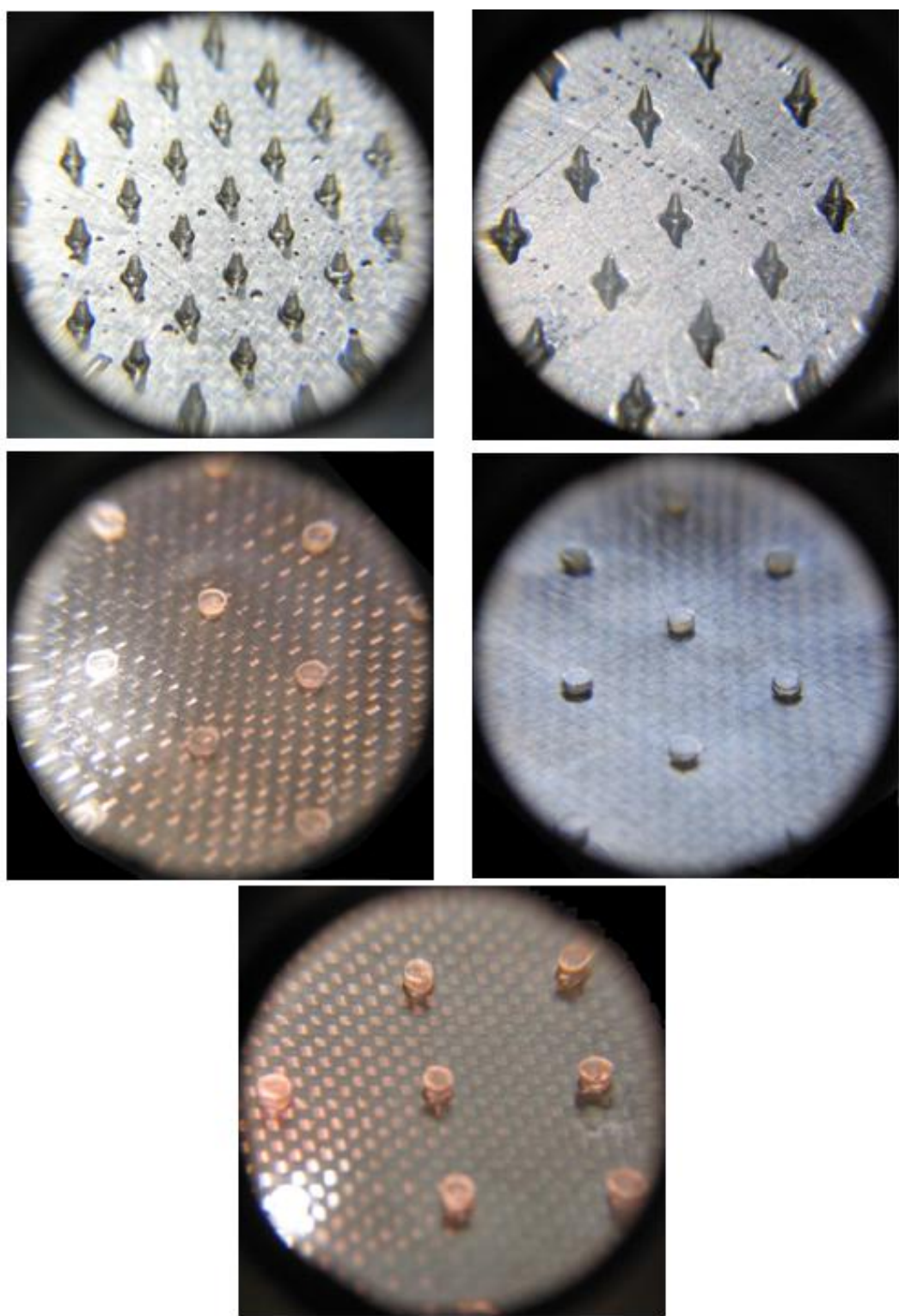

Figure 2.10: Photographs of roughness fetches. Top row: $5.49 \mathrm{~mm}$ and $8.23 \mathrm{~mm}$ spaced Gaussian elements. Middle row: $0.38 \mathrm{~mm}$ and $0.76 \mathrm{~mm}$ cylindrical elements. Bottom: $1.52 \mathrm{~mm}$ cylindrical elements. 


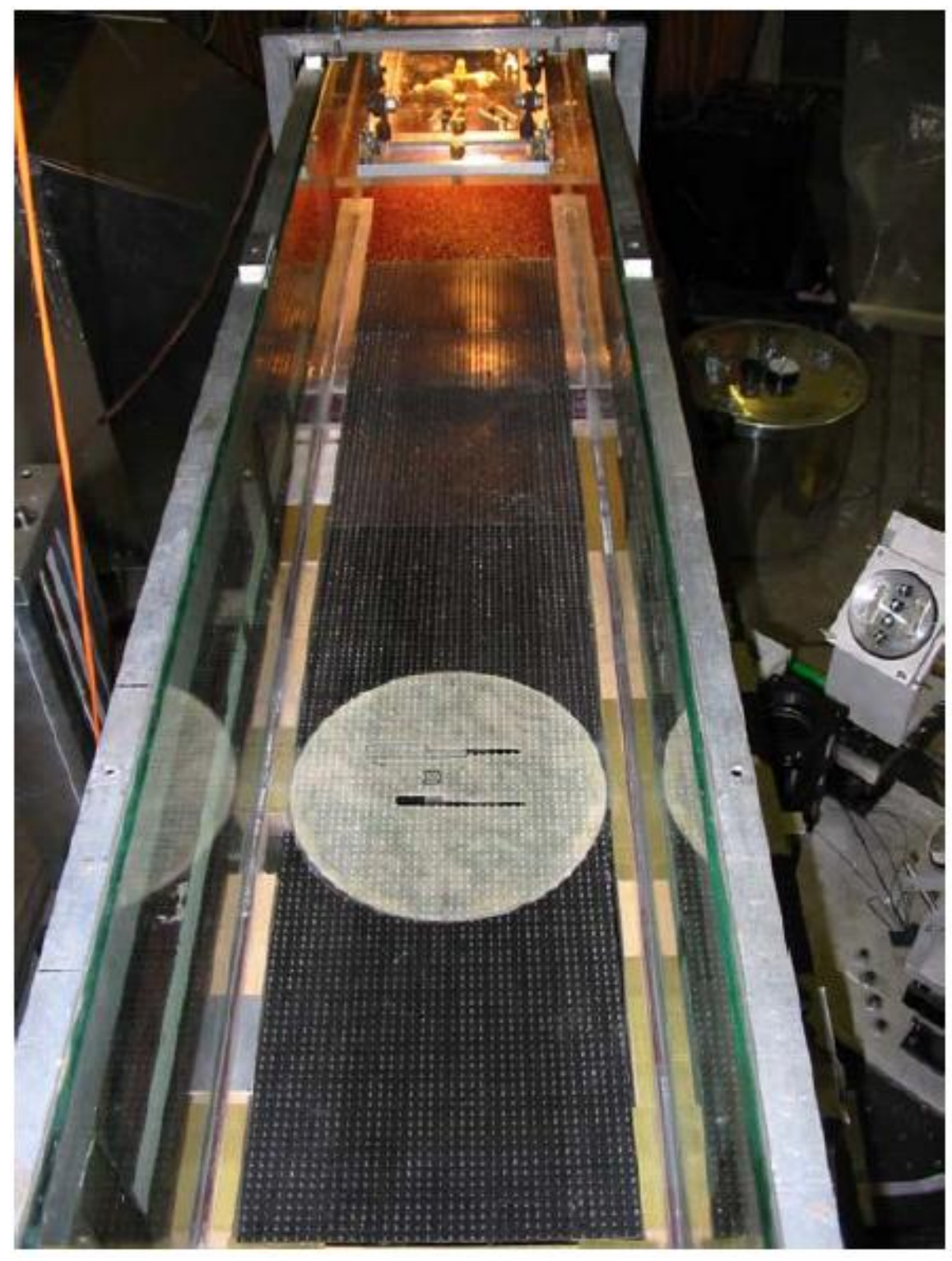

Figure 2.11: Roughness fetch installed in wind tunnel. The black area is carbon-fiber substrate fetch, and the white area is gauze substrate over the optical glass disc. Ref. Bennington (2004) 


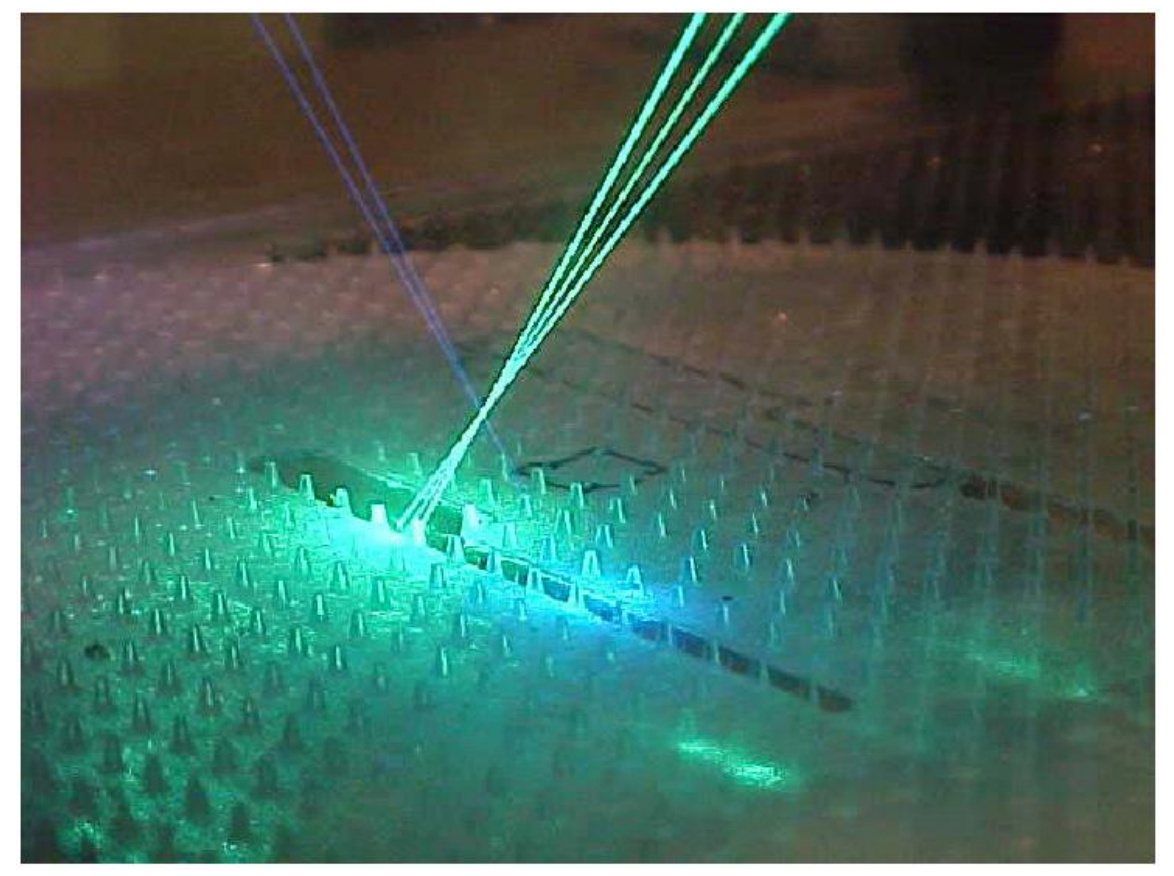

Figure 2.12: LDV measurements being taken through slot in roughness. Ref. Bennington (2004) 


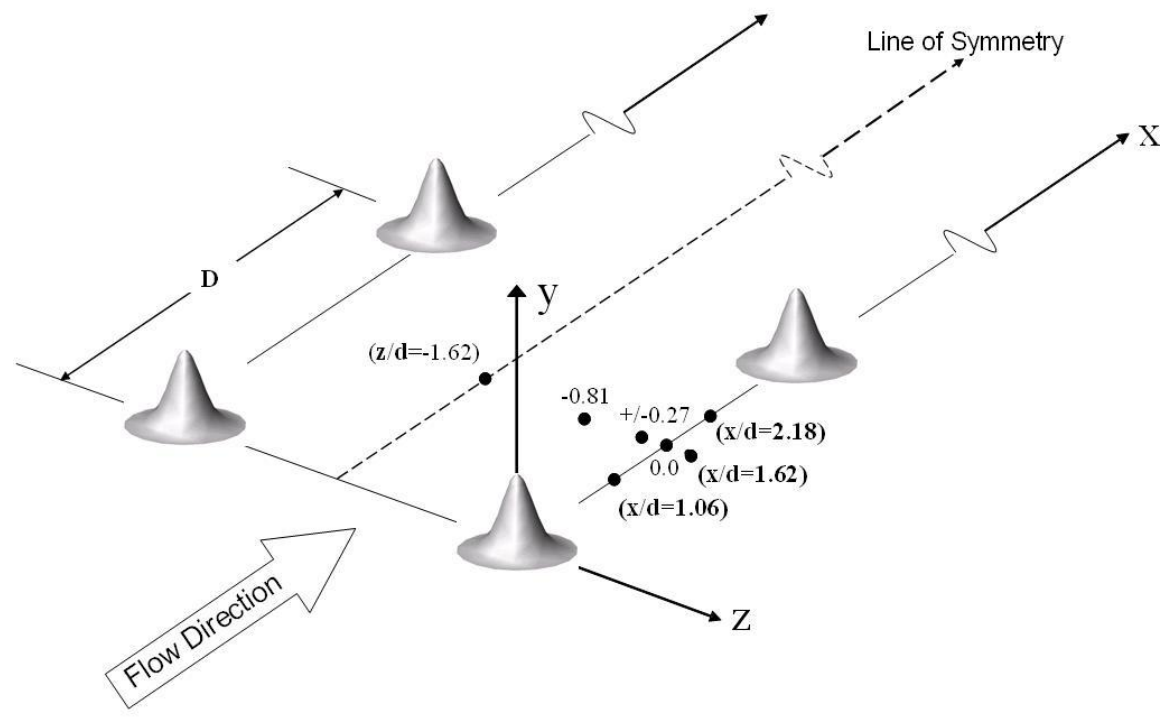

Figure 2.13: Example measurement locations for a roughness fetch in a straight configuration

2.13 (straight configuration) and 2.14 (staggered configuration). In each case, three profiles are taken which are distributed in the streamwise direction between two elements. Four more profiles are taken in the spanwise plane between two elements. The eighth profile is taken either 6 or 8 cells downstream, in order to examine the streamwise variation of the velocity profile over a larger distance. The final profile is taken by the outer layer LDV system to measure the flow beyond the range of the three-component system.

The measurement location was approximately twenty boundary layer thickness downstream of the end of the sand grain roughness (Section 2.1.1). In order to help confirm that the boundary layer was fully developed at the measurement location, a velocity profile was compared with the profile taken 6 or 8 cells downstream. The four or five outer points were compared and the average percentage difference was determined. These differences can be seen in Table 2.2. For most of the cases, the average difference was less than $5 \%$. 


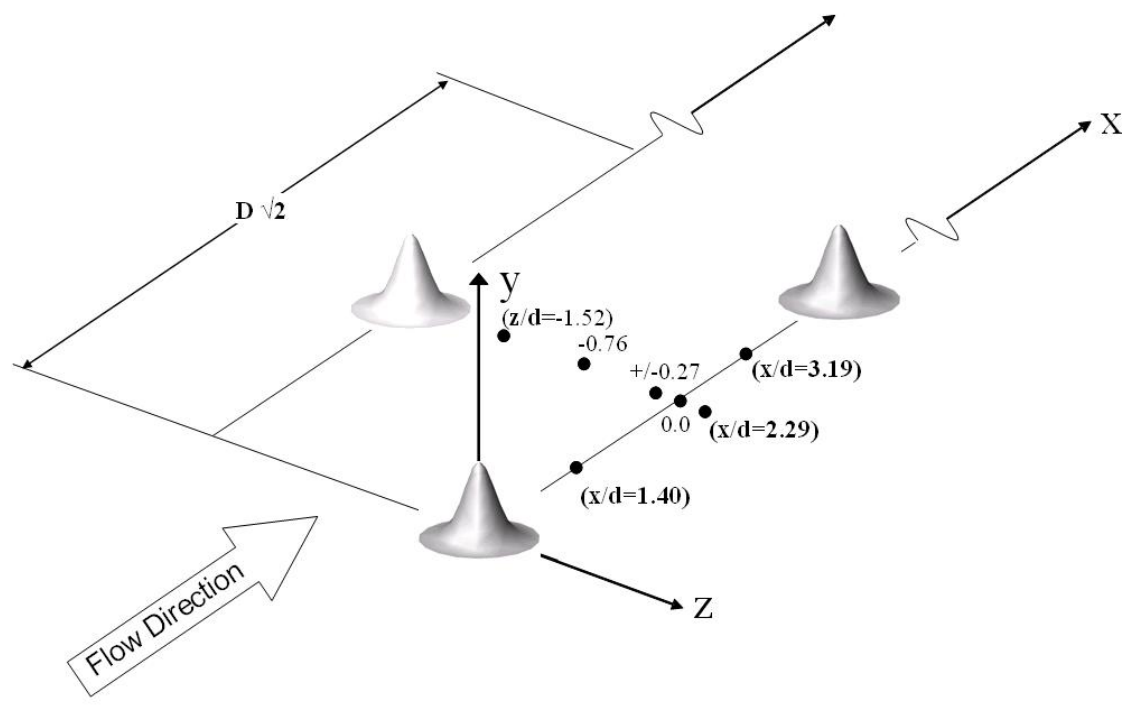

Figure 2.14: Example measurement locations for a roughness fetch in a staggered configuration. The faded element signifies that it was removed to allow access for measurements. 


\begin{tabular}{|c|c|c|}
\hline Case $\#$ & Configuration & Average Percent Difference \\
\hline \hline 1 & Gaussian, $5.49 \mathrm{~mm}$ spacing, straight & Not measured \\
\hline 2 & Gaussian, $5.49 \mathrm{~mm}$ spacing, staggered & 4.56 \\
\hline 3 & Gaussian, $8.23 \mathrm{~mm}$ spacing, straight & 3.48 \\
\hline 4 & Gaussian, $8.23 \mathrm{~mm}$ spacing, staggered & 7.05 \\
\hline 5 & $0.38 \mathrm{~mm}$ cylinder, straight & 1.47 \\
\hline 6 & $0.38 \mathrm{~mm}$ cylinder, staggered & 0.38 \\
\hline 7 & $0.76 \mathrm{~mm}$ cylinder, straight & 6.97 \\
\hline 8 & $0.76 \mathrm{~mm}$ cylinder, staggered & 2.97 \\
\hline 9 & $1.52 \mathrm{~mm}$ cylinder, straight & 1.50 \\
\hline 10 & $1.52 \mathrm{~mm}$ cylinder, staggered & 2.83 \\
\hline
\end{tabular}

Table 2.2: Average Percent Difference in Upstream and Downstream Velocity Profiles

In two cases, the average error was approximately $7 \%$. This error is largest at the measurement locations nearer to the wall, and may be due to either spatial heterogeneity in the flow just above the element or the uncertainty in the streamwise pressure gradient.

\subsubsection{Construction}

The roughness fetches consist of epoxy resin roughness elements formed by a Teflon mold and mounted on a substrate.

The mold for the Gaussian elements is a $30.5 \times 30.5 \times 0.318 \mathrm{~cm}$ sheet of Teflon, into which the Gaussian shape is drilled with an end mill cutter at the desired spacing. $0.127 \mathrm{~mm}$ vent holes extend from the peak of the spike the rest of the way through the sheet to allow air bubbles to escape during the molding process.

The molds for the cylindrical posts are $30.5 \times 30.5 \mathrm{~mm}$ Teflon sheets with a thickness equal to the desired element height. Holes of $1.98 \mathrm{~mm}$ diameter are drilled in the sheet at a spacing of $10.97 \mathrm{~mm}$.

The fetches are constructed by first spreading a mixture of epoxy resin and slow hardener over the substrate material. This substrate is generally carbon 
fiber, but thin gauze is used for the Gaussian fetch sections to be mounted over the optical glass. In the case of the cylindrical post fetches, the optical glass disc itself covered with cellophane tape is the substrate. Once the epoxy mixture is evenly spread over the substrate, the appropriate mold is pressed onto the epoxy-covered surface. Air bubbles are kneaded out from between the mold and substrate. The mold and substrate are pressed between two Plexiglass plates, and this entire assembly is placed in a bag which is then subjected to a vacuum. After three hours, the epoxy is hardened and the fetch is ready to be taken out of the vacuum bag. After removing the mold, the substrate is left with the hardened roughness elements mounted upon it. After being cut to the correct size, the fetch is attached to the tunnel floor with rubber cement. Rubber cement is easy to work with, and its adhesive properties are not adversely affected by the DOP seed material, as are some tapes and glues.

\subsubsection{Determination of Origin}

The origin of the wind tunnel coordinate system is defined in the spanwise and streamwise directions as the center of the element behind which measurements are to be taken. For the purposes of measurement, the vertical location of the origin is the surface of the glass. This will later be modified by the displacement height, as discussed in Section 1.6.

To find the center of the roughness element, a low power red laser beam is launched through the receiving fiber, so that a beam emits from the receiving optics. The traverse is moved so that the focus point of this beam is on the roughness substrate surface. This point of light, visible through the cellophane tape or gauze substrate, indicates the location of the probe measurement volume.

In the case of the Gaussian spikes, this focal point is traversed back and forth underneath the element in the spanwise direction. When the focal point is located at the peak of the spike, as determined by eye, a spanwise position reading is taken. This reading is repeated several times, and the values are averaged to determine a spanwise location of the origin. A similar process is performed, traversing upstream and downstream, to determine the streamwise location of the origin. 
Since the cylindrical posts have no precise, easily visible center point, the method for the determination of the origin is slightly different. The focal point is traversed to obtain several readings the left extreme edge of the element. These readings are averaged, and this is repeated to find the extreme rightmost edge of the cylinder. The midpoint of these edges is where the origin is located in the spanwise direction. Similarly, the streamwise location of the origin is found based on the upstream and downstream edges of the element.

Based on the scatter of the readings, the accuracy of finding the spanwise and streamwise location of the origin with this method is approximately $\pm 60 \mu \mathrm{m}$. This process is performed once for each fetch, when it is first installed, and all profile locations are based off this measurement.

The height of the glass is subject to slight variation, and the vertical measurement location needs to be determined with more precision than the horizontal location. Thus, the wall is "found" before each LDV profile is acquired. When the measurement volume is located on the wall, a strong signal is visible on a spectrum analyzer due to the flare of the laser beams at the glass/air interface. Traversing vertically, it is possible to find the wall location within $\pm 50 \mu \mathrm{m}$. 


\section{Chapter 3}

\section{Results}

\subsection{Determination of Friction Velocity}

The near wall velocities and turbulence quantities scale on the fiction velocity, $u_{\tau}$, so it is important to obtain an accurate calculation of this value. The following analysis describes the basis upon which $u_{\tau}$ was calculated in this research.

To arrive at a method for calculating $u_{\tau}$, a momentum balance is done on a control volume located on the rough wall, shown in Figure 3.1. The numbers 1 through 5 represent the surfaces of the control volume, and subscripted numbers in the following analysis refer to the value of the subscripted quantity on that surface. The side surfaces (3 and 4) are defined to be at the mid-plane between two rows of roughness elements. It can thus be assumed that the mean streamwise velocity $\bar{w}_{3,4}$ and the shear stresses $\overline{u w}_{3,4}$ are zero at these surfaces. The top of the control volume, surface 5, is set at the height where the shear stress $\overline{u v_{5}}$ is constant among all of the LDV profiles for a given roughness fetch configuration.

To begin, the integral form of the momentum equation is written for the streamwise direction

$$
\iint_{C V} \rho(\vec{V} \cdot \hat{i})(\vec{V} \cdot \hat{n}) \mathrm{d} A=-\iiint_{C V} \frac{\mathrm{d} p}{\mathrm{~d} x} \mathrm{~d} \sigma-\mathrm{F}_{x}
$$




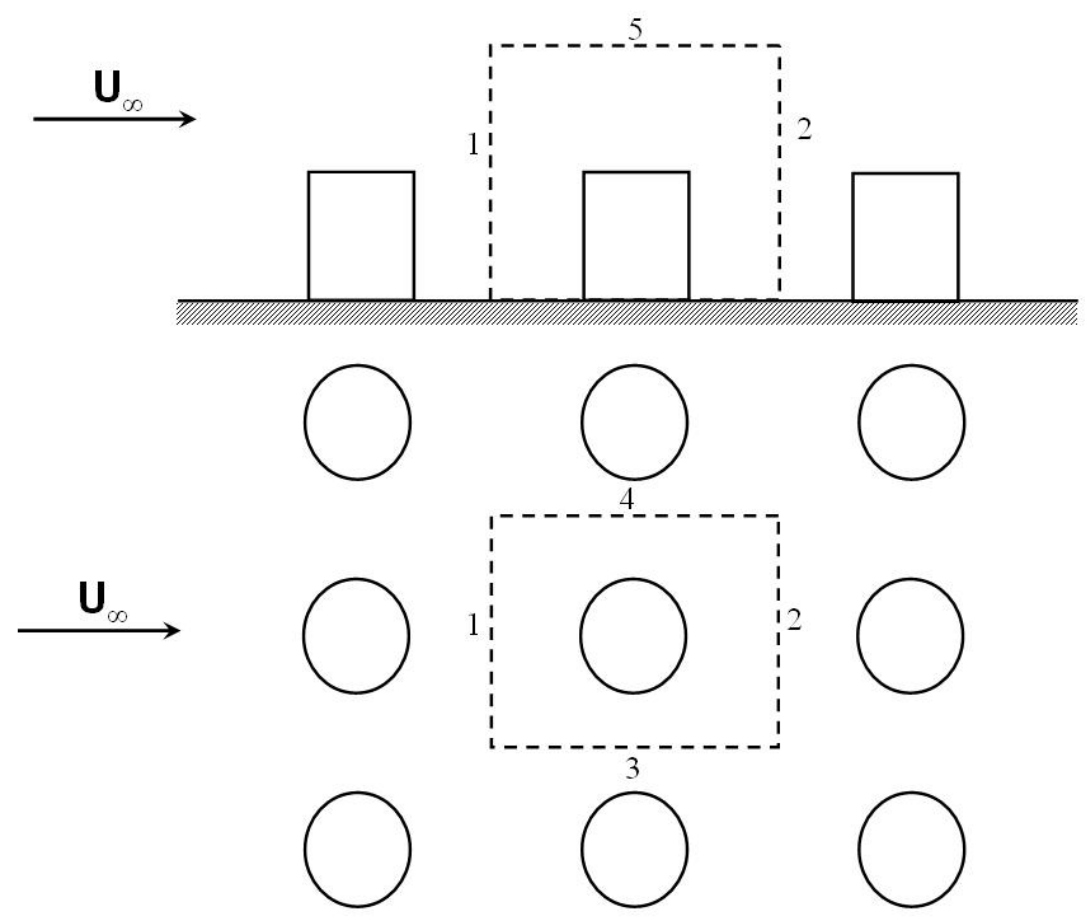

Figure 3.1: Control volume for calculation of $u_{\tau}$. Side and top views are shown, control volume surfaces are labeled 1-5. 
where $\hat{i}$ is a unit vector in the streamwise direction, $\hat{n}$ is the unit vector normal to the control volume, $\rho$ is the density of the fluid, $\mathrm{d} A$ is a differential area, $\mathrm{d} \sigma$ is a differential volume, and $\mathrm{F}_{x}$ is a force acting in the streamwise direction representing the action of friction on the control volume.

To treat the left hand side of Equation 3.1, Reynolds decomposition is performed, and the expression is time averaged

$$
\begin{array}{r}
\rho \iint_{C V}(\vec{V} \cdot \hat{i})(\vec{V} \cdot \hat{n}) \mathrm{d} A=\rho\left[\iint_{2}\left(U_{2}^{2}+\overline{u_{2}^{2}}\right) \mathrm{d} A_{2}-\iint_{1}\left(U_{1}^{2}+\overline{u_{1}^{2}}\right) \mathrm{d} A_{1}\right. \\
\left.+\iint_{5}\left(U_{5} V_{5}+\overline{u v_{5}}\right) \mathrm{d} A_{5}\right]
\end{array}
$$

Over a short distance, the pressure gradient $\mathrm{d} p / \mathrm{d} x$ is considered to be constant. Thus, the first term on the right hand side of Equation 3.1 can be expressed as

$$
-\iiint_{C V} \frac{\mathrm{d} p}{\mathrm{~d} x} \mathrm{~d} \sigma=-\frac{\mathrm{d} p}{\mathrm{~d} x} \Delta x \Delta y \Delta z=-\frac{\mathrm{d} p}{\mathrm{~d} x} V_{C V}
$$

Where $\Delta x, \Delta y$, and $\Delta z$ are the dimensions of the control volume, which has a volume $V_{C V}$.

The final term of Equation 3.1 to be dealt with is the force $\mathrm{F}_{x}$, the shear force acting on the control volume due to the presence of the wall.

$$
-\mathrm{F}_{x}=-\iint_{\text {wall }} \tau_{w} \mathrm{~d} x \mathrm{~d} z=-\rho \iint_{\text {wall }} u_{\tau}^{2} \mathrm{~d} x \mathrm{~d} z
$$

where $\tau_{w}$ is the wall shear. Over short distances, friction velocity is assumed to vary linearly in the streamwise direction. Thus, friction velocity at a downstream location is expressed as

$$
u_{\tau}=u_{\tau, 1}+\frac{\mathrm{d} u_{\tau}}{\mathrm{d} x} \Delta x
$$

where $u_{\tau, 1}$ is the value of the friction velocity at the upstream edge of the control volume. 
Plugging this definition of $u_{\tau}$ into Equation 3.4, it is shown that

$$
\begin{aligned}
-\mathrm{F}_{x} & =-\rho \iint_{\text {wall }}\left(u_{\tau, 1}^{2}+2 u_{\tau, 1} \frac{\mathrm{d} u_{\tau}}{\mathrm{d} x} \Delta x+\left(\frac{\mathrm{d} u_{\tau}}{\mathrm{d} x}\right)^{2} \Delta x^{2}\right) \mathrm{d} x \mathrm{~d} z \\
& =-\rho \Delta x \Delta z\left[\frac{\Delta x^{2}}{3}\left(\frac{\mathrm{d} u_{\tau}}{\mathrm{d} x}\right)^{2}+u_{\tau, 1} \frac{\mathrm{d} u_{\tau}}{\mathrm{d} x} \Delta x+u_{\tau, 1}^{2}\right]
\end{aligned}
$$

Using Equations 3.2, 3.3, and 3.6, momentum Equation 3.1 can be rewritten

$$
\begin{aligned}
& \iint_{2}\left(U_{2}^{2}+\overline{u_{2}^{2}}\right) \mathrm{d} A_{2}-\iint_{1}\left(U_{1}^{2}+\overline{u_{1}^{2}}\right) \mathrm{d} A_{1}+\iint_{5}\left(U_{5} V_{5}+\overline{u v_{5}}\right) \mathrm{d} A_{5} \\
& =\frac{-1}{\rho} \frac{\mathrm{d} p}{\mathrm{~d} x} V_{C V}-\Delta x \Delta z\left[\frac{\Delta x^{2}}{3}\left(\frac{\mathrm{d} u_{\tau}}{\mathrm{d} x}\right)^{2}+u_{\tau, 1} \frac{\mathrm{d} u_{\tau}}{\mathrm{d} x} \Delta x+u_{\tau, 1}^{2}\right]
\end{aligned}
$$

Several assumptions can be made to Equation 3.7 in order to simplify the calculation of $u_{\tau}$. First, the mean vertical velocity on the top surface of the control volume is assumed to be negligible $\left(V_{5}=0\right)$. As the streamwise dimension $\Delta x$ becomes small, the streamwise gradient $\frac{\mathrm{d} p}{\mathrm{~d} x}$ is assumed to be zero. By expanding $U$ and $\overline{u^{2}}$ in a Taylor series, and using an order of magnitude analysis, is can be seen that the shear stress $\overline{u v_{5}}$ balances the friction velocity. That is,

$$
u_{\tau, 1}=\sqrt{-\overline{u v_{5}}}
$$

Thus, the friction velocity is calculated directly from the value of the shear stress in the constant stress layer in the logarithmic region of the boundary layer. Table 3.1 shows the values of $u_{\tau}$ calculated with this method, along with the normalized roughness height, $k^{+}=k u_{\tau} / \nu$. 


\begin{tabular}{|c|c|c|c|}
\hline Case \# & Configuration & $u_{\tau}(\mathrm{m} / \mathrm{s})$ & $k^{+}$ \\
\hline \hline 1 & Gaussian, 5.49 mm spacing, straight & 1.43 & 217 \\
\hline 2 & Gaussian, 5.49 mm spacing, staggered & 1.50 & 228 \\
\hline 3 & Gaussian, 8.23 mm spacing, straight & 1.40 & 213 \\
\hline 4 & Gaussian, 8.23 mm spacing, staggered & 1.29 & 197 \\
\hline 5 & $0.38 \mathrm{~mm}$ cylinder, straight & 1.04 & 24 \\
\hline 6 & $0.38 \mathrm{~mm}$ cylinder, staggered & 1.01 & 23 \\
\hline 7 & $0.76 \mathrm{~mm}$ cylinder, straight & 1.11 & 51 \\
\hline 8 & $0.76 \mathrm{~mm}$ cylinder, staggered & 1.17 & 54 \\
\hline 9 & $1.52 \mathrm{~mm}$ cylinder, straight & 1.27 & 116 \\
\hline 10 & $1.52 \mathrm{~mm}$ cylinder, staggered & 1.21 & 110 \\
\hline
\end{tabular}

Table 3.1: $u_{\tau}$ and $k^{+}$values 


\subsection{Mean Velocities}

The flow structure over the six configurations of cylindrical post roughness are very similar to one another, and the flow over the four Gaussian spike arrangements are similar to each other. Thus, in order to streamline this discussion, the complete set of data is not shown here. Rather, example figures are chosen which best illustrate the behavior of the various quantities. These example figures are meant to be representative of the roughness type (cylindrical or Gaussian) as a whole. Refer to Appendix A for the complete set of plots of the roughness data.

A comparison of the mean streamwise velocities for the various fetches is shown in Figure 3.2. The profiles represent the average of the LDV profiles taken for a given roughness configuration, along with the data taken by the Outer Layer LDV system. The downward shift $\Delta U / u_{\tau}$ of the rough wall profiles from the smooth wall reference is apparent. As is expected, smaller elements with a wider spacing produce a smaller shift than the larger elements with tighter spacings. This effect and correlations predicting the roughness effect will be discussed in depth in Chapter 4 .

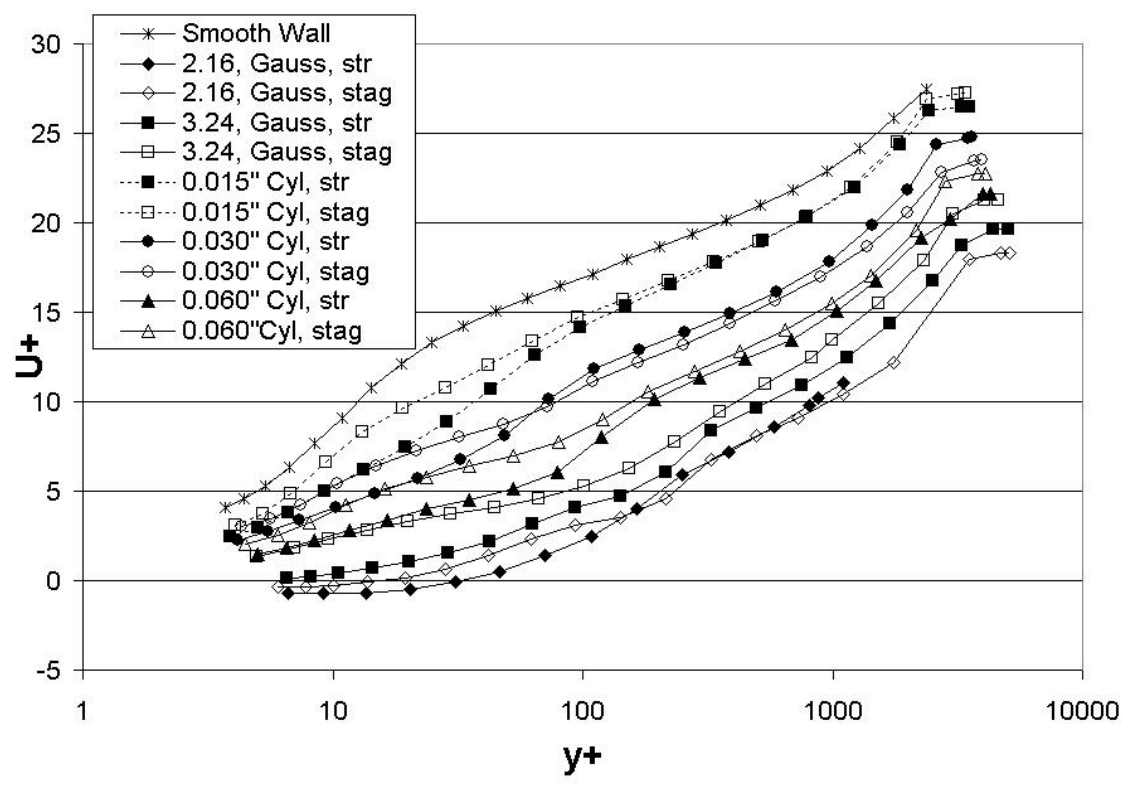

Figure 3.2: Comparison of spatially averaged mean velocity profiles 
Representative plots of centerline mean velocities are shown for three cases in Figure 3.3. The centerline refers data taken on the $x$-axis, the streamwise line emanating from the center of a roughness element. The normalized roughness height, $k^{+}=k u_{\tau} / \nu$, is represented by a vertical dashed line. In each case, an area of near wall separation is evident from the negative streamwise velocities seen in the profiles closest to the upstream roughness element. The velocity is progressively higher in the two profiles located farther downstream, as the flow recovers from the wake behind the element. The larger elements exhibit a more pronounced backflow region, as evidenced by the size and magnitude of the negative velocity region. Near the top of the roughness element, the shielding effect of the roughness element from the oncoming flow disappears, and a steep velocity gradient is visible. The streamwise velocity profiles collapse between 1.4 and 2 roughness heights, and above this point are approximately identical to each other. 

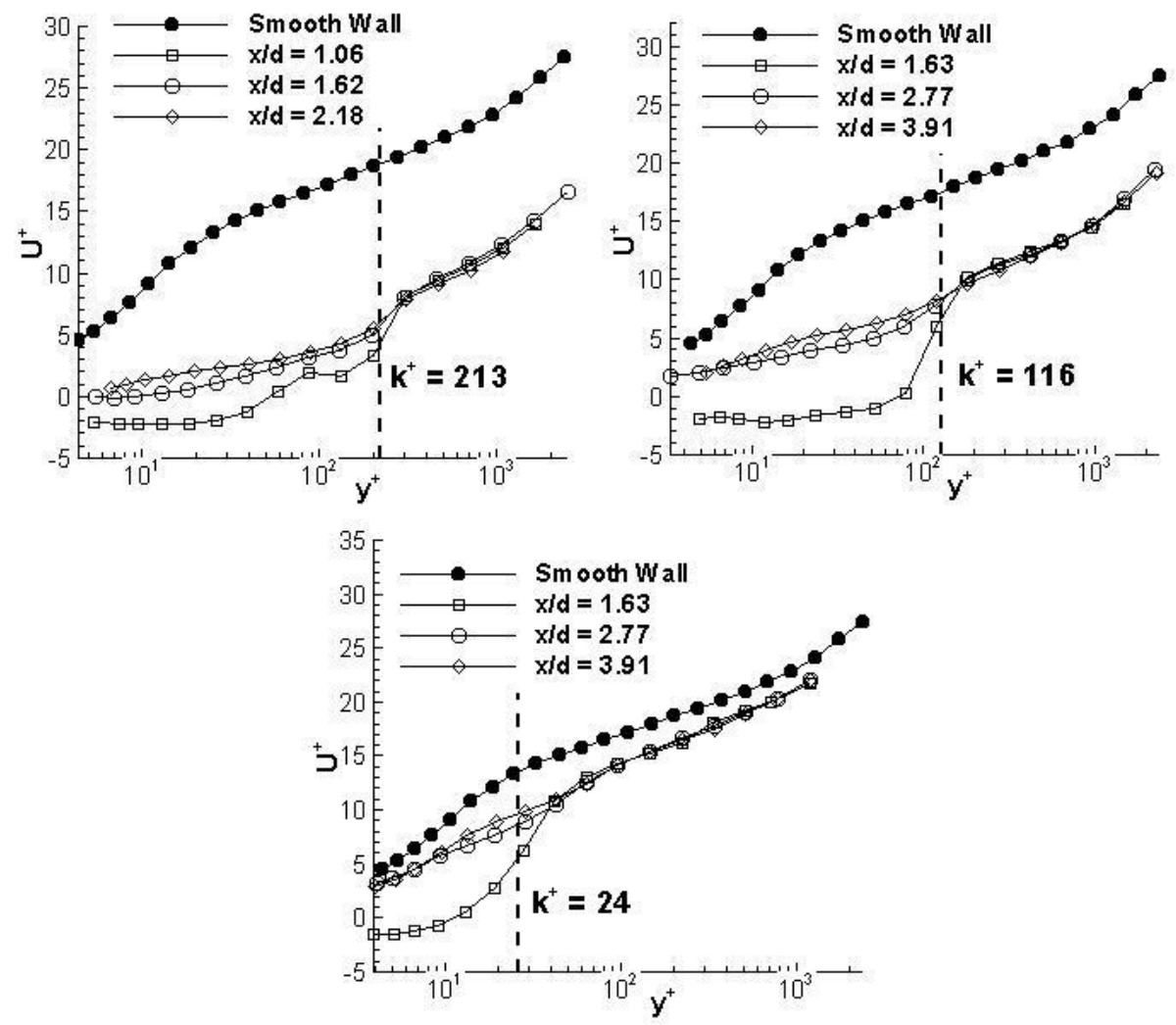

Figure 3.3: Example centerline streamwise mean velocities. Top left: 8.23 mm spacing, Gauss, straight (Case \#3). Top right: $1.52 \mathrm{~mm}$ cylinder, straight (Case \#9). Bottom: 0.38 mm cylinder, straight (Case \#5).

Figure 3.4 shows velocity profiles taken in the spanwise, vertical plane between two rows of roughness elements, referred to as the mid-plane. Once again, the shielding effect of the roughness element is evident. Below the roughness height, the profiles located behind the element have lower velocities than the profiles located farther to the side of the element. Fluid in the gaps between roughness elements is faster flowing, and it feeds into the separated flow regions behind the element. At the level of the roughness height, the profiles to the sides of a roughness element do not experience the drastic velocity gradient that the profiles behind the element do. It can be seen again that the mean velocity profiles collapse approximately between 1.4 and 2 roughness heights. 

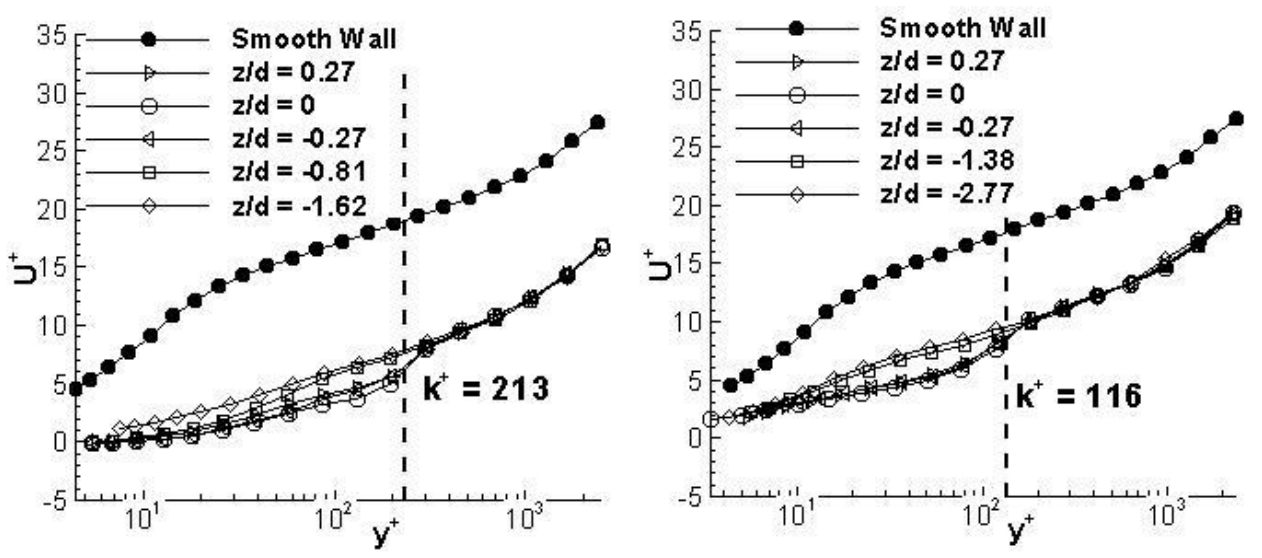

Figure 3.4: Example mid-plane streamwise mean velocities. Left: $8.23 \mathrm{~mm}$ spacing, Gauss, straight (Case \#3). Right: $1.52 \mathrm{~mm}$ cylinder, straight (Case \#9).

Figure 3.5 shows examples of the mean vertical velocity profiles on the centerline. The Gaussian spike exhibits two negative peaks, one located at approximately $25 \%$ of the roughness height, and the second at or near the roughness height. The profile just downstream of the Gaussian element $(x / d=1.06)$ has a small region of positive $V^{+}$at $60 \%$ of the roughness height, indicating that there is an area of upward flow in the separation region directly behind the element. In the data of ?, this effect can be seen more clearly since profiles were taken closer to the upstream element. As will be discussed later, this region of upward flow is associated with the ejection of low-speed fluid away from the wall. This region of positive normal-to-wall velocity does not appear in the profiles taken farther downstream.

Velocity data for the cylindrical element fetches shown in Figure 3.5 are markedly different than that of the Gaussian elements. There is only one maximum point of negative $V^{+}$velocity, located at approximately the roughness height. The profiles nearest to the upstream element have the greatest magnitude, as a result of the flow coming down over the top of the element. The $\mathrm{x} / \mathrm{d}=1.63$ profile shows a small upwash region directly behind the large cylinder, at $20 \%$ of the element height. In the case of the small and medium height cylinders, this region was probably smaller and closer to the element, so it was not able to be measured and does not appear in that data. 

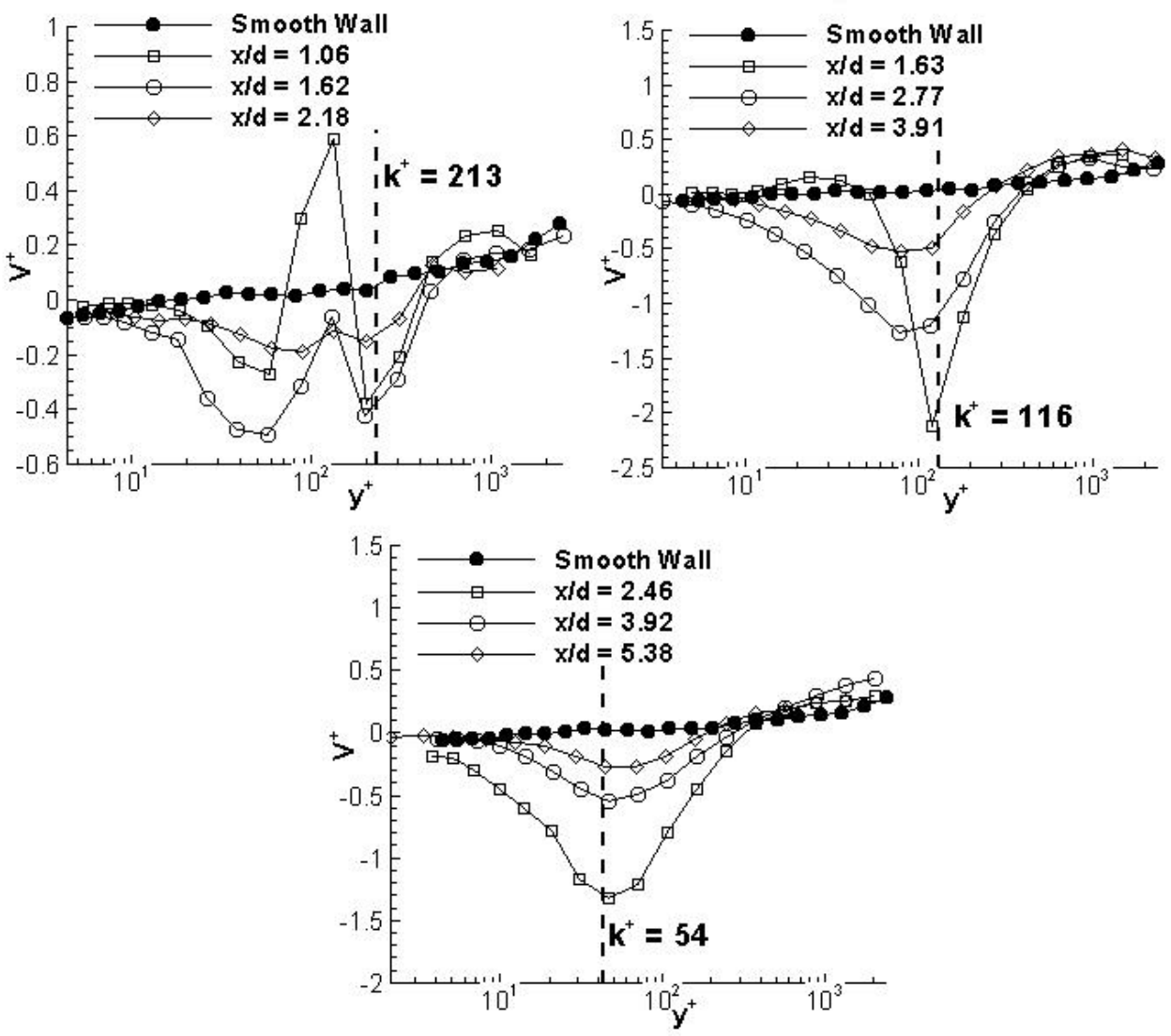

Figure 3.5: Example centerline vertical mean velocities. Top left: $8.23 \mathrm{~mm}$ spacing, Gauss, straight (Case \#3). Top right: $1.52 \mathrm{~mm}$ cylinder, straight (Case \#9). Bottom: $0.76 \mathrm{~mm}$ cylinder, staggered (Case \#8).

The difference in the $V$ velocity profiles between the cylindrical and Gaussian elements is important to note. The cylindrical elements both show large magnitude peaks near the element height, while the Gaussian elements have multiple peaks, of comparatively smaller magnitude. Data presented in following sections will exhibit similar behavior, indicating that the flow around the cylinders is typified by turbulent mixing in a thin layer near the element height, while the Gaussian spikes have activity over a broader region that extends well below the element peaks.

Examples of mean spanwise velocity $W^{+}$are plotted for the mid-plane in 
Figure 3.6. In the Gaussian spike data, velocities are negative in the positive $z$-direction, and vice versa, indicating that the flow is coming around the element and in from the sides toward the centerline. Near the wall $(\approx 20 \%$ of the roughness height), the the profiles to either side of the centerline $(z / d= \pm 0.27)$ indicate mean velocities away from the centerline, while near the roughness height, the mean velocity is toward the centerline.
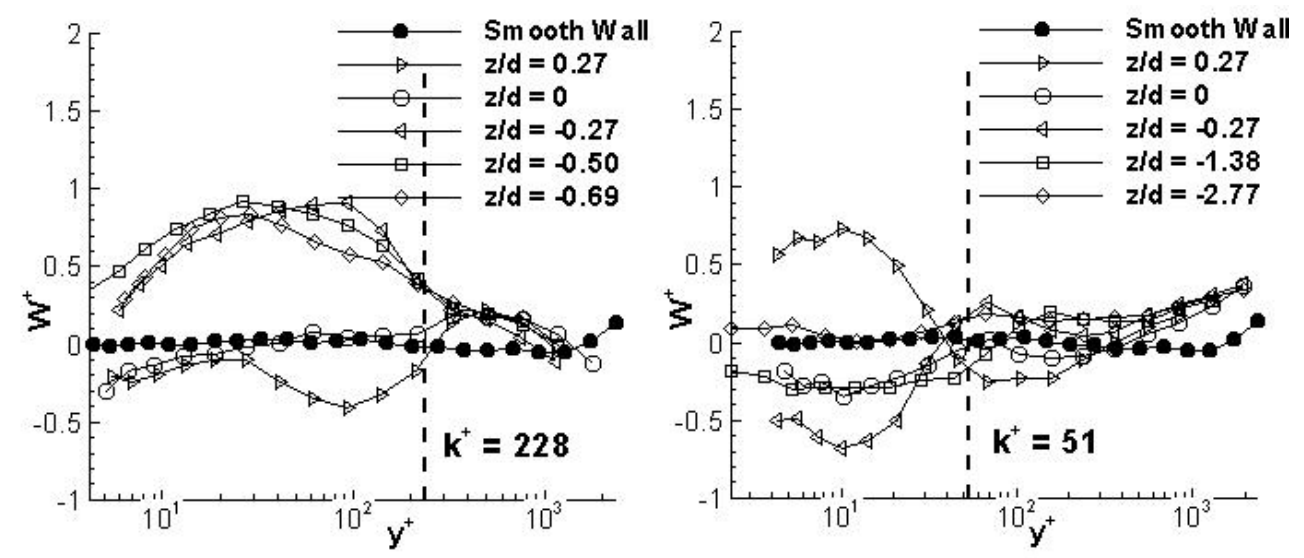

Figure 3.6: Example mid-plane spanwise mean velocities. Left: $5.49 \mathrm{~mm}$ spacing, Gauss, staggered (Case \#2). Right: $0.76 \mathrm{~mm}$ cylinder, straight (Case \#7).

Taken together, these observations of the mean velocities indicate there is a low speed separation region behind the elements, whose size relates to the size of the element. Higher speed fluid comes down over the top and around the sides of the elements. The flow pattern below the roughness height varies significantly with position, but above the roughness elements the velocity profiles quickly collapse. 


\subsection{Reynolds Stresses and Turbulent Kinetic Energy}

In subsequent sections, it will be seen that the highest levels of turbulence occur in the vicinity of the element height, for both cylindrical and Gaussian elements. This is due to the interface between the low momentum fluid near the wall below the element height, and the relatively high speed fluid above the elements. Accordingly, many turbulence quantities show either relatively large positive or negative maxima near the element height. However, data for the Gaussian roughness fetches often have additional large maxima or minima below the roughness height, while the cylindrical fetches do not. This distinction is attributed to the difference in the geometry of the two types of elements. The flat top of the cylindrical element creates a thin region where the majority of the turbulent mixing occurs. Profiles of turbulence quantities around cylindrical elements display sharp, single peak values at the element height. Gaussian elements, on the other hand, have a sharply peaked top, and significant turbulence is present over a fairly broad region. There is no sharp division between the fluid shielded by the elements, and the higher speed fluid above the elements. Rather, the size of the region protected from oncoming flow dwindles as the Gaussian element tapers to its peak. Profiles of turbulence quantities for the Gaussian spikes have maxima at multiple vertical locations, with lower magnitudes than those of the cylindrical elements. Though the maximum turbulence still occurs at the peak of the Gaussian element, significant turbulence also exists below the roughness height.

Reynolds stresses and turbulent kinetic energy (TKE) data demonstrate that the area of greatest turbulence in a rough wall flow is at the height of the roughness element. This is a region of intense mixing, where the higher speed fluid from above the roughness comes into contact with the lower speed fluid that is amongst the elements, and trapped in the low speed separations directly behind the elements. Examination of the Reynolds normal stresses shows that the $\overline{u^{2}} / u_{\tau}^{2}$ stress, which is predominant on a smooth wall, is greatly reduced in the rough wall cases. The $\overline{v^{2}} / u_{\tau}^{2}$ and $\overline{w^{2}} / u_{\tau}^{2}$ stresses are conversely much greater in the rough wall configuration. Reynolds stresses reach their maximum magnitude near the roughness height, and are greatest in the separation region directly behind a roughness element. 
Centerline plots of $\overline{u^{2}} / u_{\tau}^{2}$ Reynolds normal stresses are shown in Figure 3.7. The smooth wall profile exhibits a large peak near the wall, at a location of $y^{+}=14$. The presence of the roughness largely suppresses the $\overline{u^{2}} / u_{\tau}^{2}$ stress near the wall, as seen in all four examples in Figure 3.7. The rough wall $\overline{u^{2}} / u_{\tau}^{2}$ profiles reach their maxima at approximately 1 to 1.5 roughness heights. The profiles located immediately downstream of the roughness element have the largest magnitude of $\overline{u^{2}} / u_{\tau}^{2}$, which decreases in subsequent profiles. Likewise, plots of $\overline{u^{2}} / u_{\tau}^{2}$ stress in the mid-plane show that profiles taken behind the element exhibit higher magnitudes than those taken away from the centerline.
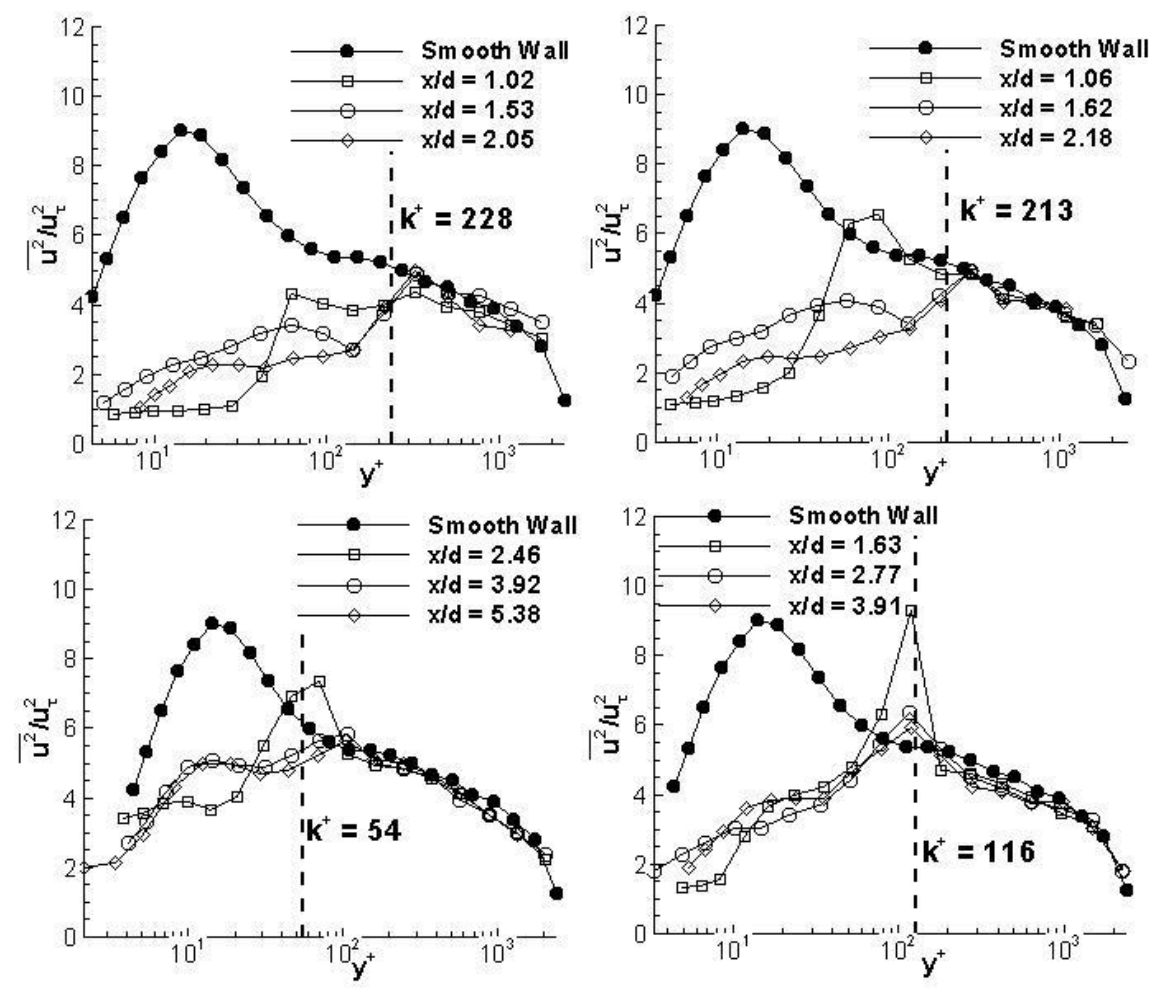

Figure 3.7: Example centerline $\overline{u^{2}} / u_{\tau}^{2}$ normal stresses. Top left: $5.49 \mathrm{~mm}$ spacing, Gauss, staggered (Case \#2). Top right: $8.23 \mathrm{~mm}$ spacing, Gauss, straight (Case \#3). Bottom left: $0.76 \mathrm{~mm}$ cylinder, staggered (Case \#8). Bottom right: $1.52 \mathrm{~mm}$ cylinder, straight (Case \#9).

Examples of $\overline{v^{2}} / u_{\tau}^{2}$ and $\overline{w^{2}} / u_{\tau}^{2}$ normal stresses can be seen in Figure 3.8. For the smooth wall, $\overline{v^{2}} / u_{\tau}^{2}$ and $\overline{w^{2}} / u_{\tau}^{2}$ are much smaller than $\overline{u^{2}} / u_{\tau}^{2}$ stresses. 
By comparison, Figure 3.8 shows these stresses to be large for the rough wall. Flow over the top and around the sides of an element bring in larger $v$ and $w$ fluctuations as compared to $u$ fluctuations. The peaks for the $\overline{v^{2}} / u_{\tau}^{2}$ stress occur typically at 0.9 to 1.5 roughness heights, while the maxima for $\overline{w^{2}} / u_{\tau}^{2}$ occur slightly lower, in the vicinity of $60 \%$ to $90 \%$ of the roughness height.
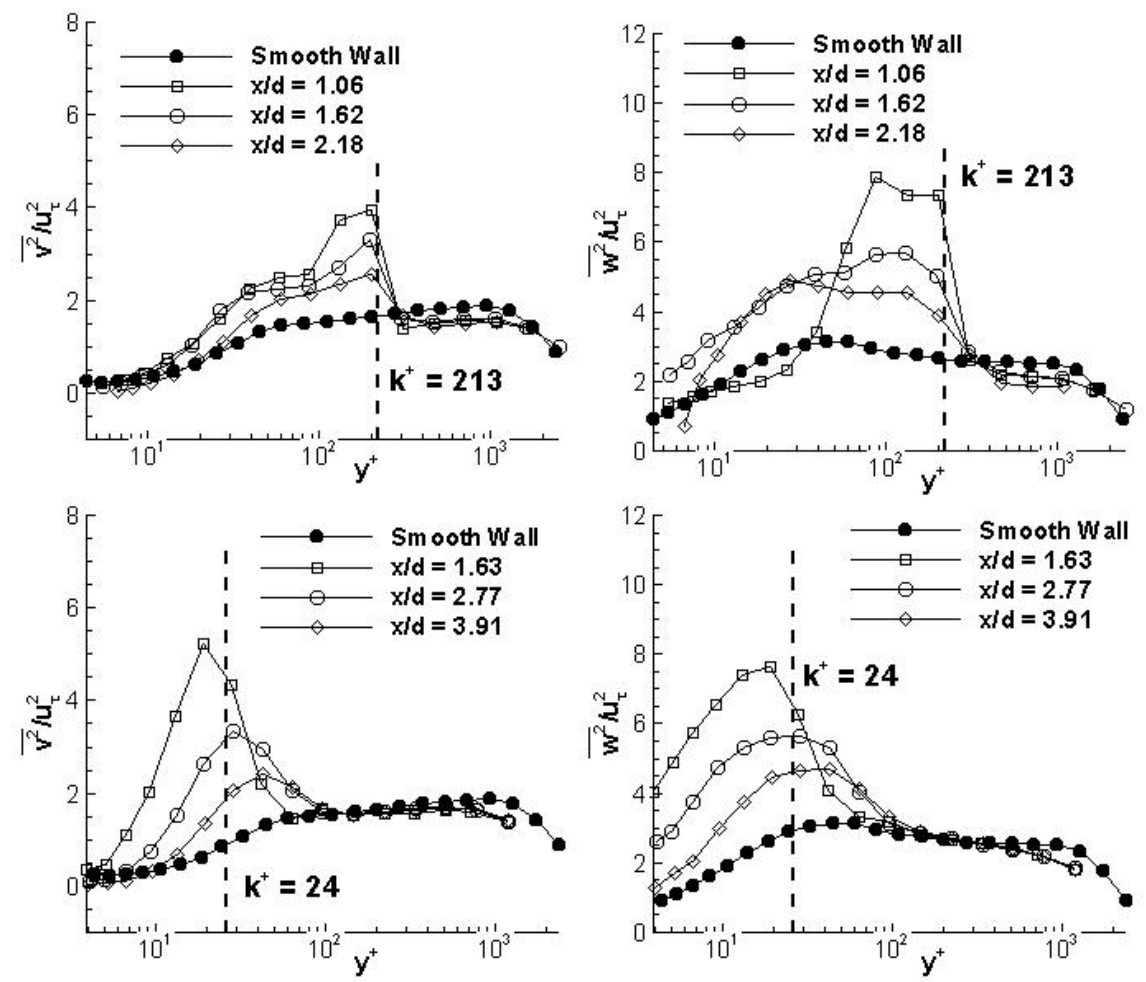

Figure 3.8: Example centerline $\overline{v^{2}} / u_{\tau}^{2}$ and $\overline{w^{2}} / u_{\tau}^{2}$ normal stresses. Top: $8.23 \mathrm{~mm}$ spacing, Gauss, staggered (Case \#4). Bottom: $0.38 \mathrm{~mm}$ cylinder, straight (Case \#5).

The mid-plane plots of $\overline{v^{2}} / u_{\tau}^{2}$ and $\overline{w^{2}} / u_{\tau}^{2}$ in Figure 3.9 show that the effect of the roughness on these normal stresses is largely isolated to the region directly behind the element. The profiles taken farther from the centerline closely follow the smooth wall profile. Figure 3.9 also shows the collapse of the normal stress profiles at approximately 2 to 4 roughness heights. In the outer layer, the normalized rough wall profiles closely follow that of the smooth wall profile. 

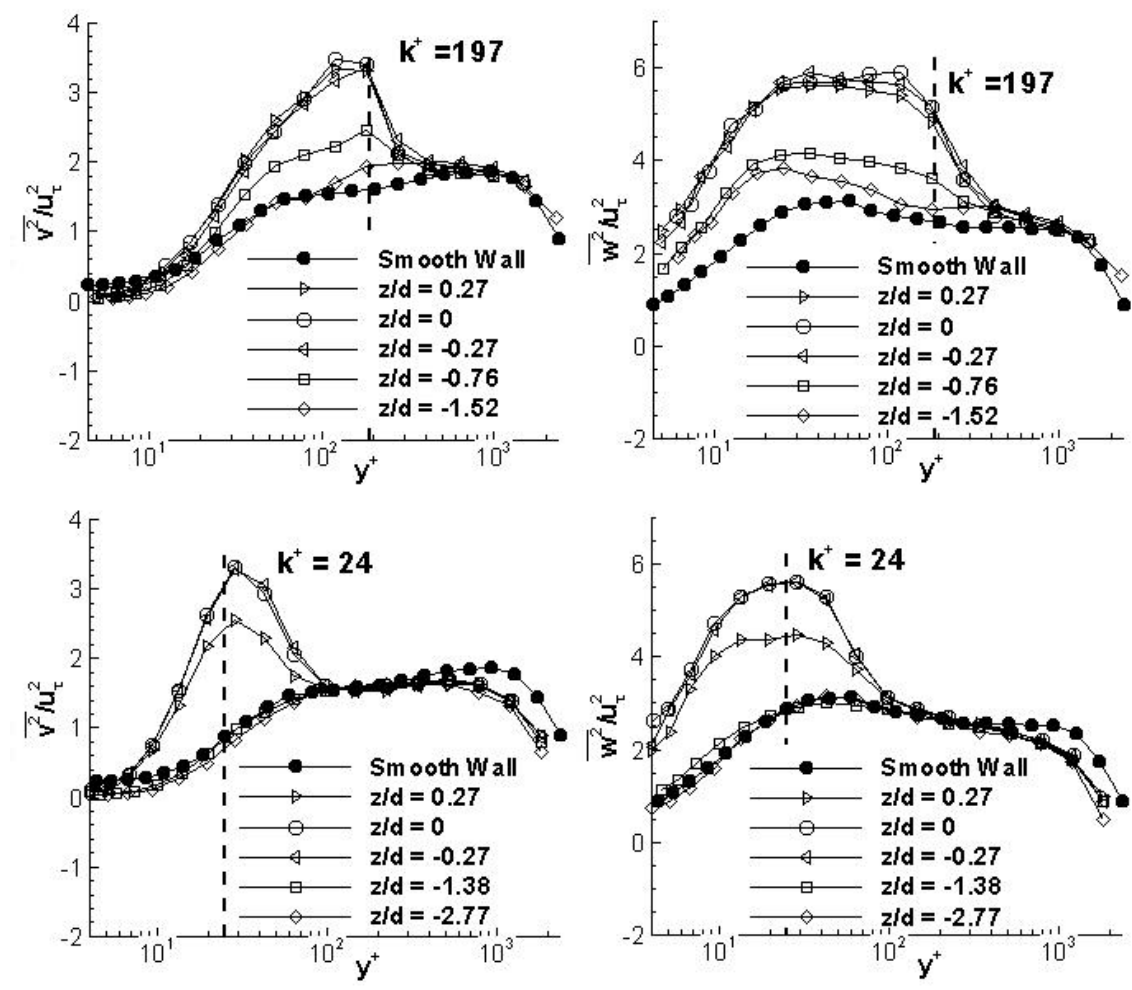

Figure 3.9: Example mid-plane $\overline{v^{2}} / u_{\tau}^{2}$ and $\overline{w^{2}} / u_{\tau}^{2}$ normal stresses. Top: $8.23 \mathrm{~mm}$ spacing, Gauss, staggered (Case \#4). Bottom: $0.38 \mathrm{~mm}$ cylinder, straight (Case \#5).

Several examples of the Reynolds shear stress $-\overline{u v} / u_{\tau}^{2}$ are plotted in Figure 3.10. The plots for Gaussian elements, shown in the top two plots, have a positive peak at half the roughness height, and a negative peak near the roughness height. When compared to the smooth wall profile, it can be seen that there are lower shear stress levels near the wall, but higher levels at the roughness height.

There is only one peak in the $\overline{u v} / u_{\tau}^{2}$ shear stress for cylindrical roughness, seen in the bottom two plots of Figure 3.10. The maximum shear stress magnitude occurs near the roughness height. The magnitude of this peak is much larger than the magnitude of the shear stresses on the Gaussian roughness. This is due to the comparatively larger magnitude of the $\overline{v^{2}}$ 
stress, and the large velocity gradient $\partial U / \partial y$, which leads to a larger $-\overline{u v}$ production rate, equal to $\overline{v^{2}} \frac{\partial U}{\partial y}$.
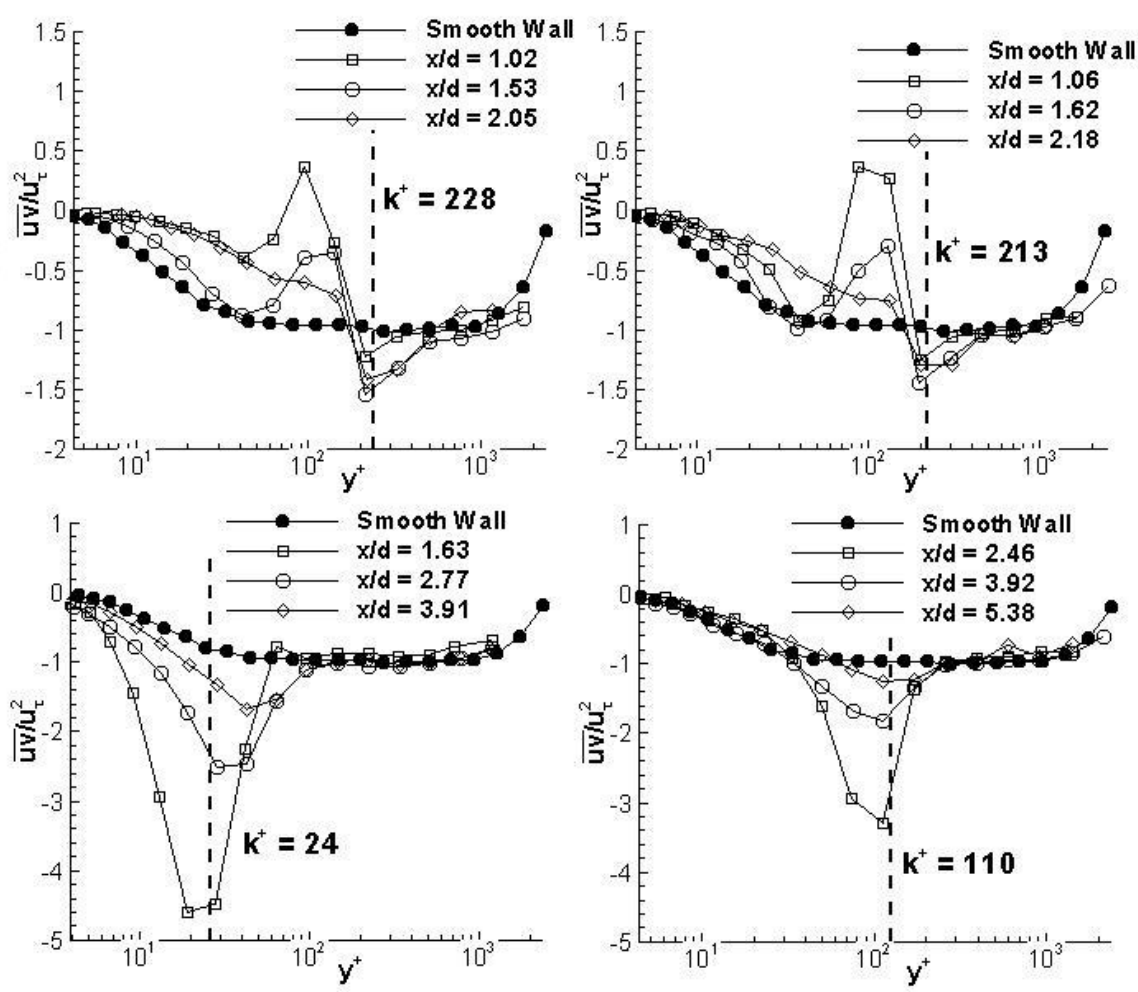

Figure 3.10: Example centerline $\overline{u v} / u_{\tau}^{2}$ shear stresses. Top left: $5.49 \mathrm{~mm}$ spacing, Gauss, staggered (Case \#2). Top right: $8.23 \mathrm{~mm}$ spacing, Gauss, straight (Case \#3). Bottom left: $0.38 \mathrm{~mm}$ cylinder, straight (Case \#5). Bottom left: $1.52 \mathrm{~mm}$ cylinder, staggered (Case \#10).

The turbulent kinetic energy (TKE) is a function of the sum of the Reynolds normal stresses,

$$
T K E=\frac{1}{2} \frac{\overline{u^{2}}+\overline{v^{2}}+\overline{w^{2}}}{u_{\tau}^{2}}=\frac{1}{2} \frac{\overline{q^{2}}}{u_{\tau}^{2}}
$$

Thus, the behavior of the rough wall TKE profiles echoes that of the normal stress profiles. The TKE generally reaches its maximum in the vicinity of the roughness height. 
Example mid-plane plots are shown in Figure 3.11. The TKE normalized by $u_{\tau}^{2}$ is much greater than that of the smooth wall for profiles taken in or near the separation region. However, profiles taken away from the separation region have similar magnitudes of TKE as the smooth wall values.
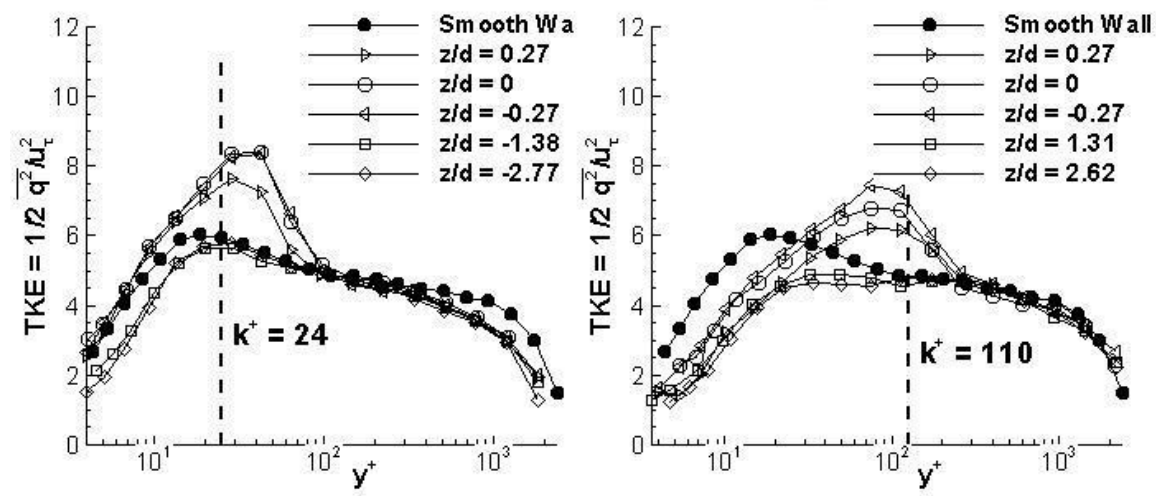

Figure 3.11: Example mid-plane TKE data. Left: $0.38 \mathrm{~mm}$ cylinders, straight (Case \#5). Right: $1.52 \mathrm{~mm}$ cylinder, straight (Case \#9).

Element spacing has a significant effect on the magnitudes of Reynolds stresses and TKE present in the flow. For a given element shape, the maximum magnitudes of these quantities are greater for a larger spacing. This is supported both by comparing the data of this author and Bennington (2004) taken on the $5.49 \mathrm{~mm}$ and $8.23 \mathrm{~mm}$ spaced Gaussian elements, as well as by comparing the $10.97 \mathrm{~mm}$ spaced cylinder data of this author and the 5.49 mm spaced cylinder data of George (2005).

To analyze trends in turbulence quantities related to element spacing, the maximum measured magnitude of a given quantity is compared with the maximum corresponding to the same case at the larger spacing, and the percent increase (or decrease) in that maximum is calculated.

The four cases involving the smaller, $0.38 \mathrm{~mm}$ and $0.76 \mathrm{~mm}$, cylinders show less of an increase in the maximum measured value of turbulence quantities for increasing spacing than do the $1.52 \mathrm{~mm}$ cylindrical element fetches. For the cases with smaller cylindrical elements $(0.38$ and $0.76 \mathrm{~mm})$, there is only a slight increase, less than $10 \%$, in $\overline{u^{2}} / u_{\tau}^{2}$ with the increased spacing. However, the $1.52 \mathrm{~mm}$ cases show a larger increase of approximately $40 \%$. The $0.38 \mathrm{~mm}$ and $0.76 \mathrm{~mm}$ elements show an approximately $40 \%$ increase in 
$\overline{v^{2}} / u_{\tau}^{2}$, while the $1.52 \mathrm{~mm}$ cylinders approximately double in magnitude with the larger spacing. All three sets of cylinders have a considerable increase in $\overline{w^{2}} / u_{\tau}^{2}$ normal stress, of approximately $20-30 \%$. The cumulative effect of this is that 0.38 and $0.76 \mathrm{~mm}$ cylinders have a $30-50 \%$ greater maximum TKE for the larger spacing, while the $1.52 \mathrm{~mm}$ cylinders have an $80 \%$ increase in maximum TKE. Similarly, the $\overline{u v} / u_{\tau}^{2}$ shear stress shows a large increase of $60-80 \%$ for the $1.52 \mathrm{~mm}$ cylinders, while the smaller cylinders show a lesser increase of $15-30 \%$ for the larger spacing.

For the data on the Gaussian fetches, there is little difference in the measured maximum $\overline{u^{2}} / u_{\tau}^{2}$ normal stress between the $5.49 \mathrm{~mm}$ and $8.23 \mathrm{~mm}$ spaced cases. In both the straight and staggered orientations, there is a 50 $\%$ increase in $\overline{v^{2}} / u_{\tau}^{2}$ and a $20 \%$ increase in $\overline{w^{2}} / u_{\tau}^{2}$ for the larger spacing. The maximum measured TKE is $45 \%$ greater for the larger spaced elements. The data does not show any significant trend in the $\overline{u v} / u_{\tau}^{2}$ shear stress for the different element spacings. 


\subsection{Triple Products}

Triple products can provide an indication of the relative dominance of sweeping motions of fluid toward the wall, or ejection motions away from the wall. Though a full octant analysis is necessary to show the magnitude and fraction of time that these events are present, triple products give an overall picture of the motion of the fluid.

Figure 3.12 shows the triple products $\overline{u^{3}} / u_{\tau}^{3}$ and $\overline{u^{2} v} / u_{\tau}^{3}$ for the mid-plane of the $0.76 \mathrm{~mm}$ cylinders in the straight configuration. These plots are representative of the behavior of these quantities on the cylindrical fetches. Near the wall, $\overline{u^{3}} / u_{\tau}^{3}$ is positive, while $\overline{u^{2} v} / u_{\tau}^{3}$ is negative. This implies that a negative $v$ fluctuation is dominant, and $u$ fluctuations are being brought toward the wall in a sweeping motion. In the three profiles behind the element $(z / d=0, \pm 0.27)$ there is a sharp negative peak in the $\overline{u^{3}} / u_{\tau}^{3}$ profile, and a large positive peak in the $\overline{u^{2} v} / u_{\tau}^{3}$ profile near the roughness height. These peaks denote the prevalence of negative $u$ and positive $v$ events, indicating the ejection of low-momentum fluid along the centerline behind the element. This effect is greatly reduced in the two profiles taken farther away from the centerline.
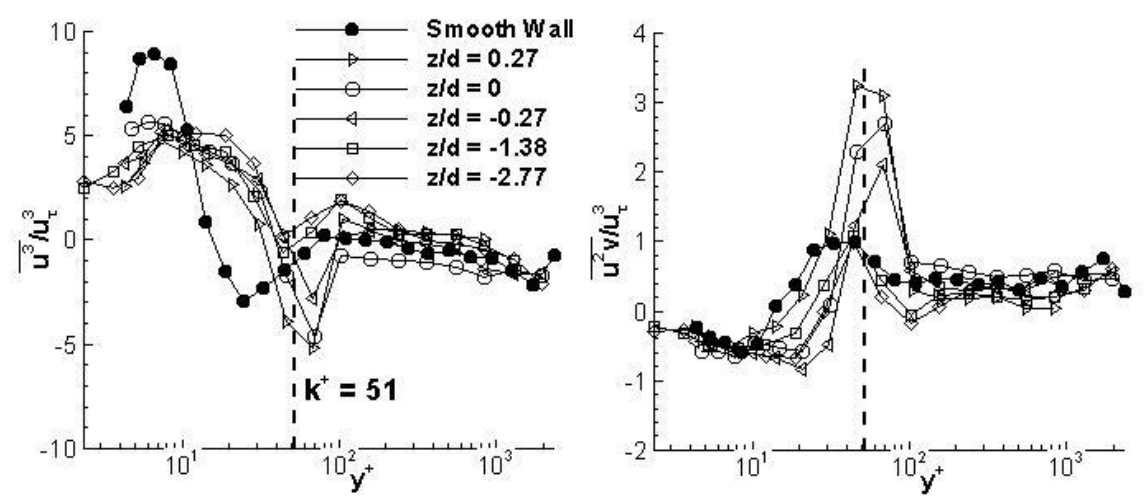

Figure 3.12: Mid-plane $\overline{u^{3}} / u_{\tau}^{3}$ and $\overline{u^{2} v} / u_{\tau}^{3}$ for $0.76 \mathrm{~mm}$ cylinder, straight (Case \#7).

The $\overline{u^{3}} / u_{\tau}^{3}$ and $\overline{u^{2} v} / u_{\tau}^{3}$ triple products show different behavior on the Gaussian element fetches compared to the cylindrical roughness. Profiles for the $8.23 \mathrm{~mm}$ spaced, straight Gaussian spikes are shown in Figure 3.13. The 
$\overline{u^{3}} / u_{\tau}^{3}$ profile remains positive below the roughness height, with a peak near the element height. The $\overline{u^{2} v} / u_{\tau}^{3}$ is negative very close to the wall $\left(y^{+}<50\right)$, has a positive peak at $y^{+} \approx 70$, and has a negative peak near the roughness height. This indicates that while positive $u$ fluctuations are dominant near the wall, the sign of $v$ fluctuates. There is a region near the wall dominated by the sweeps of $u$ fluctuations, and a second region of sweeps located near the element height (as opposed to ejections, as in the case of the cylindrical elements). In the case of both the cylindrical and Gaussian roughness, $\overline{u^{3}} / u_{\tau}^{3}$ is negative in the outer layer, and $\overline{u^{2} v} / u_{\tau}^{3}$ is positive, indicating the ejection events are prevalent.
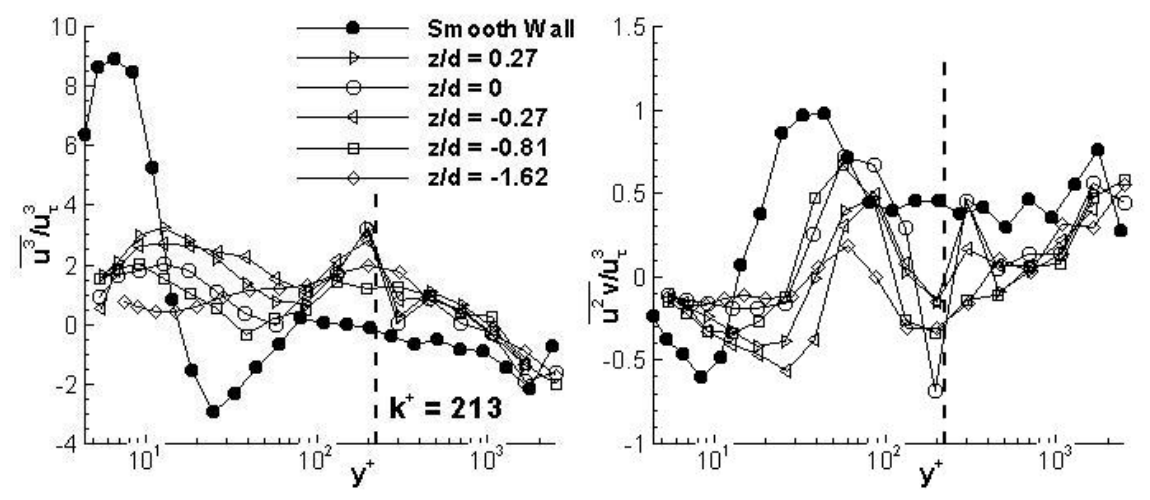

Figure 3.13: Mid-plane $\overline{u^{3}} / u_{\tau}^{3}$ and $\overline{u^{2} v} / u_{\tau}^{3}$ for $8.23 \mathrm{~mm}$ spacing, Gauss, straight (Case \#3).

The $\overline{v^{3}} / u_{\tau}^{3}, \overline{u v^{2}} / u_{\tau}^{3}$, and $\overline{v^{2} w} / u_{\tau}^{3}$ plots for the mid-plane of the $1.52 \mathrm{~mm}$ straight cylinders are shown in Figure 3.14, which is representative of the cylindrical fetches as a whole. The plot of $\overline{v^{3}} / u_{\tau}^{3}$ is negative near the wall for profiles directly behind the element, and zero for profiles away from the centerline. The profiles then have a large positive peak at the element height. In the plot of $\overline{u v^{2}} / u_{\tau}^{3}$, those same profiles show a positive peak near the wall $\left(y^{+} \approx 40\right)$, and a negative peak at the element height. Thus, it is seen that sweep events dominate near the wall on the centerline, bringing $v$ fluctuations toward the wall. Near the element's peak, the downstream flow is dominated by ejections. The plot of $\overline{v^{2} w} / u_{\tau}^{3}$ only shows significant magnitudes in the $z / d= \pm 0.27$ profiles, indicating that the dominant $w$ fluctuation pushes $\overline{v^{2}} / u_{\tau}^{2}$ momentum away from the centerline. 

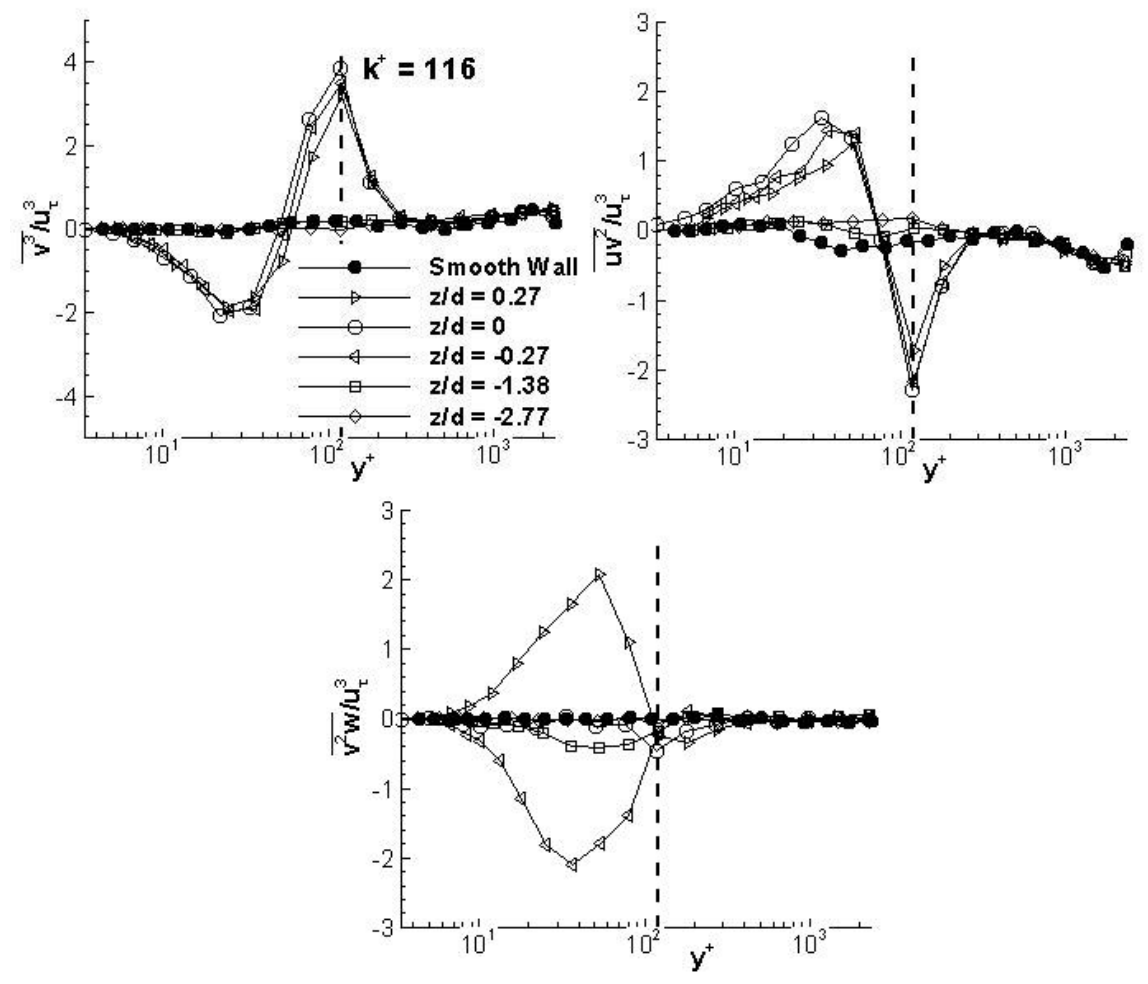

Figure 3.14: Mid-plane $\overline{v^{3}} / u_{\tau}^{3}, \overline{u v^{2}} / u_{\tau}^{3}$, and $\overline{v^{2} w} / u_{\tau}^{3}$ for $1.52 \mathrm{~mm}$ cylinders, straight (Case \#9).

Mid-plane plots of $\overline{v^{3}} / u_{\tau}^{3}$ and $\overline{u v^{2}} / u_{\tau}^{3}$ for the $8.23 \mathrm{~mm}$ spaced straight Gaussian elements are shown in Figure 3.15. Once again, the behavior of these triple products is significantly different than that of the cylinders. Two regions of sweeps are visible, near $y^{+}=30$ and $y^{+}=120$. There is a region of ejections near $y^{+}=70$, and a second just above the roughness height.

Examination of the triple products shows that the area behind the cylindrical roughness elements and below the roughness height is dominated by the sweep of high-momentum fluid toward the wall. Near the roughness height, ejection is the dominant motion. The Gaussian roughness fetches are distinctly different, showing two areas of sweeps and ejections. In all cases, ejections dominate the outer region of the boundary layer. Similar behavior was observed by George (2005), in that sweeps dominated the near wall regions of the flow and ejection events dominated in the region near the 
element heights and into the outer layers. Note that while triple products show the overall effects of sweep and ejection motions, a full octant analysis would have to be done to show the magnitude of the contributions of these events. 

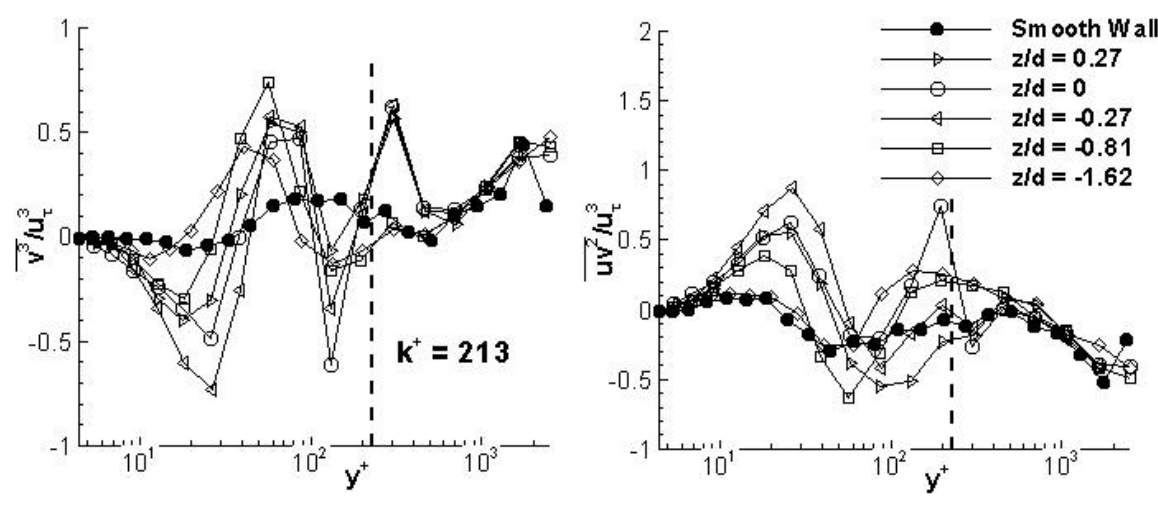

Figure 3.15: Mid-plane $\overline{v^{3}} / u_{\tau}^{3}, \overline{u v^{2}} / u_{\tau}^{3}$ for $8.23 \mathrm{~mm}$ spacing, Gauss, straight (Case \#3). 


\section{Chapter 4}

\section{Discussion and Analysis}

\subsection{Introduction}

This chapter is intended to draw upon the results described in Chapter 3 and to try to systematically characterize the effects of roughness on the velocity profile. First, an overall discussion of the flow around the roughness elements will be given, in order to summarize the observations from Chapter 3. The remainder of the chapter addresses the characteristics of the mean streamwise $U$ velocity profile, as determined by fitting the experimental data to a model equation. Trends in the curve-fitting variables will be discussed. These variables include the displacement height, $\epsilon$, the roughness effect, $\Delta U / u_{\tau}$, and the wake parameter, $\Pi$.

\subsection{Summary of Rough Wall Flow Behavior}

A cartoon of the flow around roughness elements, adapted from George (2005), is shown in Figure 4.1. This illustration is based on the data presented in Chapter 3, along with the data taken by George (2005) on a finer measurement grid.

The presence of the roughness elements distributed over a wall creates a low momentum near wall region. This is evidenced by the low streamwise 


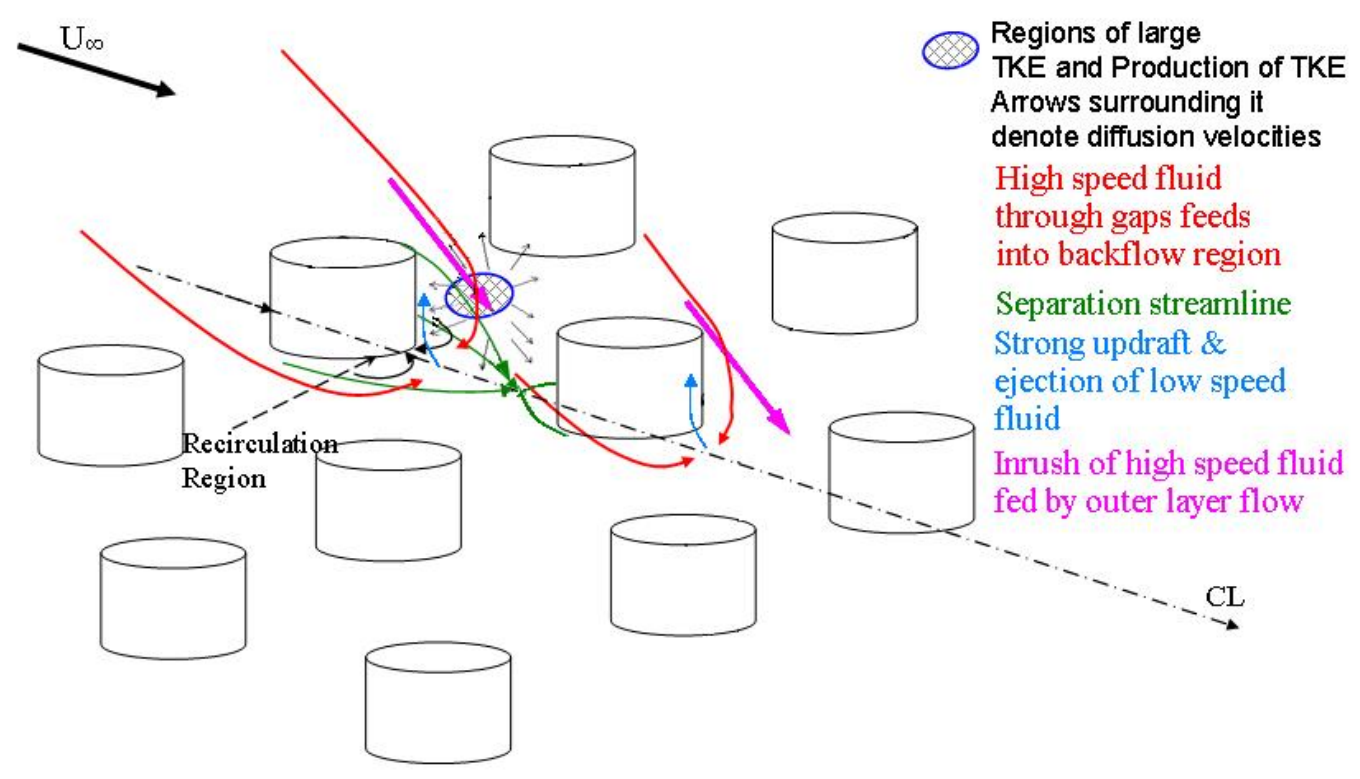

Figure 4.1: Illustration of flow near roughness elements. Adapted from George (2005).

velocities measured below the roughness height (For example, see Figures 3.3 and 3.4). High levels of turbulent stress and turbulent kinetic energy are formed near the top of the roughness elements at the shear layer formed by the interaction of the low momentum near-wall fluid and the higher speed fluid above the roughness elements.

The flow immediately downstream of a roughness element is dominated by a recirculation region. In Figure 4.1, green mean separation streamlines indicate this area, along with the small separation region upstream of the element. High momentum fluid is brought to this region in two ways. As indicated by the red arrows, higher speed fluid from upstream of the elements comes through the gaps between the elements, and rushes in behind the elements from the sides. High speed fluid also comes from over the top of the elements, brought toward the wall in strong sweeping motions. Experimental data show negative normal-to-wall mean velocities in the turbulent shear layer, and triple products indicate sweeping motions of turbulent stresses towards the wall. These events are indicated by the purple arrows in Figure 4.1. The flow around the sides and over the top of the roughness elements 
contribute much larger $v$ and $w$ fluctuations, as compared to the $u$ fluctuations. This effect is seen by the large magnitude of the $\overline{v^{2}} / u_{\tau}^{2}$ and $\overline{w^{2}} / u_{\tau}^{2}$ normal stresses, as opposed to $\overline{u^{2}} / u_{\tau}^{2}$ stresses.

On the downstream side of the roughness element, there is a small region where low momentum fluid is ejected. Shown in Figure 4.1 by pale blue arrows, this fluid is drawn upwards by the high speed fluid passing over the top of the element. In the elements with large separation regions, such as the Gaussian spikes and the $1.52 \mathrm{~mm}$ cylinders, this area of upwash can be seen in the profiles taken closest to the downstream side of the element.

The area indicated by the cross-hatched ellipse in Figure 4.1 denotes a shear layer at the interface of the low and high momentum fluid. Experimental data show high levels of turbulence and turbulent kinetic energy in this region. George (2005) notes that this is the region of highest production of turbulent kinetic energy, and the diffusion velocities $V_{q}=\overline{v q^{2}} / \overline{q^{2}}$ and $W_{q}=\overline{w q^{2}} / \overline{q^{2}}$ show the transport of energy away from this region. The gray arrows around the ellipse indicate the direction of diffusion velocities.

Though the elements shown in Figure 4.1 are cylinders, this illustration is an accurate qualitative depiction of the behavior of the flow around Gaussian spikes as well as cylinders. Though both roughness types have generally the same flow features, the magnitudes of mean and turbulence quantities differ. It is proposed that these differences occur as a result of the specific element geometry. The flow coming over the flat top of the cylinder produces a well-defined mixing layer at the roughness element height. Thus, the measurements taken on the cylindrical roughness fetches are characterized by sharp, strong peaks in turbulence levels near the roughness height. On the other hand, the smaller, peaked tops of the Gaussian elements do not provide as clearly-defined an interface between the near wall flow below the roughness height and the higher momentum fluid above. The result is a more "muddled" mixing region, shown in the experimental data by a thicker region of elevated turbulence levels. This turbulence is more distributed through the near wall region, and does not have peaks of the same large magnitude as are visible in the cylindrical element data. 


\subsection{Boundary Layer Characteristics}

In order to characterize and quantify the effect of the roughness on the boundary layer mean velocity profile, a model equation is fit to the experimental data. That model equation, Equation 4.1, is a modified form of the the laws of the wall and wake, presented previously as Equations 1.2 and 1.5.

$$
\frac{U}{u_{\tau}}=\frac{1}{\kappa} \ln \left(\frac{\left(y^{\prime}-\epsilon\right) u_{\tau}}{\nu}\right)+C-\frac{\Delta u}{u_{\tau}}+\frac{\Pi}{\kappa} W\left(\frac{y^{\prime}-\epsilon}{\delta}\right)
$$

In Equation 4.1, $\kappa$ and $C$ are assumed to be constants, given the commonly accepted values of 0.40 and 5.1 , respectively. The calculation of the skin friction velocity $u_{\tau}$ was discussed in Section 3.1, and values for each roughness case are presented in Table 3.1.

The boundary layer thickness $\delta$ denotes the vertical location at which the mean streamwise velocity is $99 \%$ of the free-stream velocity:

$$
\delta=\left.y\right|_{U=.99 U_{\infty}}
$$

The boundary layer thickness was determined by an interpolation of the data obtained by the Outer Layer LDV system. A parabola with zero slope at the free-stream was fit to three or four of the Outer Layer LDV data points. Calculated values are presented in Table 4.1.

Therefore, there are three quantities to be determined: displacement height $\epsilon$, the roughness effect $\Delta U$, and the wake parameter $\Pi$. These three quantities are iterated in Equation 4.1 to maximize the correlation coefficient with the experimental data. The results for each quantity will be discussed in subsequent sections.

To help minimize the effect of any spatial heterogeneity of the rough wall flow and experimental uncertainty, the data to which Equation 4.1 was fit was a spatial average of the several LDV profiles taken for each case. The average velocity at each vertical location $y_{i}$ is the average of the velocity measured at that location in each profile

$$
U_{\text {ave }}\left(y_{i}\right)=\overline{U\left(y_{i}\right)}
$$


This spatially averaged method was checked by fitting individual LDV profiles and averaging the resulting values of $\epsilon, \Delta U$, and $\Pi$. The values agreed well with those obtained by the spatially averaged profile in most cases (standard deviations were typically within 2.5, .3 and .08 for $\epsilon, \Delta U$, and $\Pi$, respectively), and the correlation coefficient was higher for the fit of average profile than that of any individual profile. Individual profiles were used to calculate curve fit values in cases \#3,5 and 8 , because results obtained by the averaged profiles were poor.

In the cylindrical cases, Outer Layer LDV data was not used to determine the wake parameter, since this system is subject to larger uncertainties, and sufficient data was measured in the wake region by the 3-D LDV system. However, in the Gaussian cases, data could not be taken as far into the wake region with the 3-D LDV, so Outer Layer LDV data was used in the curve fit of the wake parameter. The difference in values of $\Pi$ with or without Outer Layer LDV was less than 0.06 .

Table 4.1 presents several calculated quantities for the various roughness cases. The boundary layer thickness, along with the ratio of the roughness height to boundary layer thickness, $k / \delta$, are shown. The importance of $k / \delta$ will be discussed in a later section. The Reynolds number based on momentum thickness, $R e_{\theta}$, is also presented. $\theta$ was calculated using the spatial averaged LDV profiles, along with the Outer Layer LDV data, in the equation

$$
\theta=\int_{0}^{\delta}\left(1-\frac{U}{U_{\infty}}\right) \frac{U}{U_{\infty}} \mathrm{d} y
$$

\subsection{Determination of Displacement Height}

In Chapter 1, Section 1.6, the displacement height $\epsilon$ was discussed. The displacement height defines the location of the effective wall, as illustrated in Figure 4.2. The distance from the effective wall is therefore defined as

$$
y=y^{\prime}-\epsilon
$$

To determine the displacement height, two methods have been used. In 


\begin{tabular}{|c|c|c|c|c|c|}
\hline Case \# & Configuration & $\delta(\mathrm{mm})$ & $k / \delta$ & $\Pi$ & $R e_{\theta}$ \\
\hline \hline 1 & Gaussian, 5.49 mm spacing, straight & 44.2 & 0.057 & 1.15 & 10532 \\
\hline 2 & Gaussian, 5.49 mm spacing, staggered & 43.8 & 0.058 & 1.05 & 11359 \\
\hline 3 & Gaussian, 8.23 mm spacing, straight & 49.2 & 0.052 & 0.97 & 11786 \\
\hline 4 & Gaussian, 8.23 mm spacing, staggered & 47.7 & 0.053 & 1.09 & 11733 \\
\hline 5 & $0.38 \mathrm{~mm}$ cylinder, straight & 38.8 & 0.010 & 0.66 & 8746 \\
\hline 6 & $0.38 \mathrm{~mm}$ cylinder, staggered & 40.4 & 0.009 & 0.57 & 9569 \\
\hline 7 & $0.76 \mathrm{~mm}$ cylinder, straight & 42.7 & 0.018 & 0.70 & 10471 \\
\hline 8 & $0.76 \mathrm{~mm}$ cylinder, staggered & 46.6 & 0.016 & 0.61 & 10383 \\
\hline 9 & $1.52 \mathrm{~mm}$ cylinder, straight & 50.5 & 0.030 & 0.80 & 11309 \\
\hline 10 & $1.52 \mathrm{~mm}$ cylinder, staggered & 43.2 & 0.035 & 0.67 & 11089 \\
\hline
\end{tabular}

Table 4.1: Boundary layer thickness $\delta, k / \delta$, Wake parameter $\Pi$, and momentum thickness Reynolds number $R e_{\theta}$.

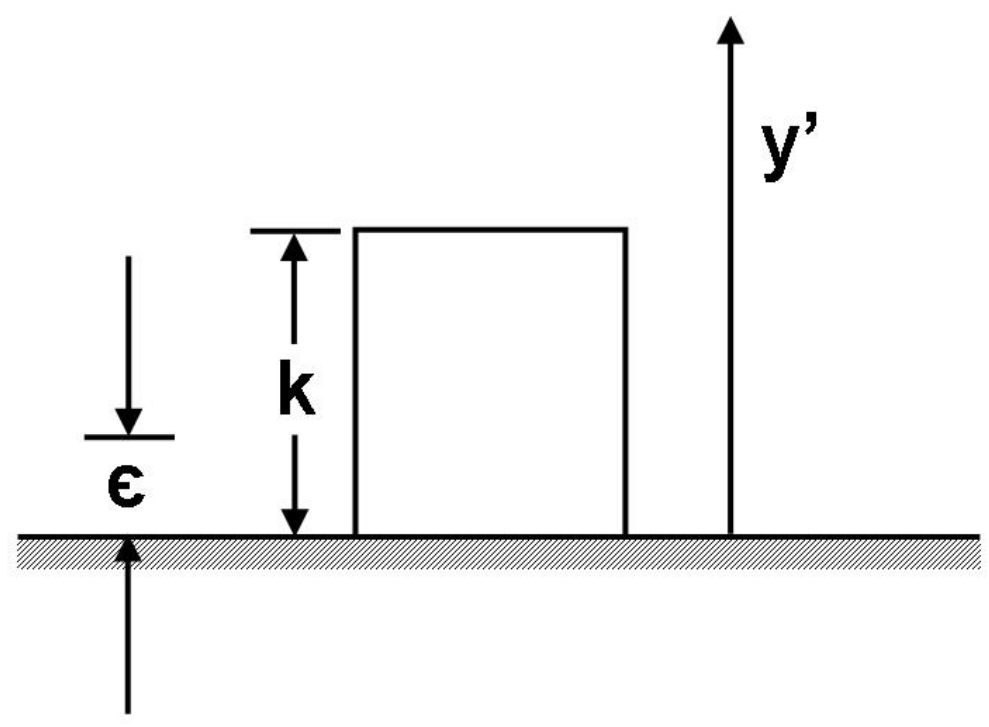

Figure 4.2: Illustration of displacement height 
the first, displacement height is found by matching Cole's Law of the Wall to the experimental data, as described in Section 4.3.

For the second method, $\epsilon$ is determined by a method which is an extension of that of Schlichting (1936). In his data reduction, Schlichting spread the volume of the roughness elements out evenly to form a smooth wall. Thus, a smooth wall flow with the same volume of fluid as the rough wall flow was formed. However, this method does not account for any effect of the roughness elements on the surrounding flow. To account for the effects of the roughness in this analysis, the volume of the separated flow regions near the roughness element have been included when determining the displacement height. This is a more physically sound method than that of Schlichting (1936), since continuity requires that the flow streamlines be offset by the volume of both the roughness element and that of the separated flow regions.

Clearly, a very low uncertainty estimate of the mean separation volume is difficult to obtain without detailed measurements at many more locations than obtained here. This is a small, near-wall region, and even the detailed LDV measurements taken do not clearly define this volume. Therefore, the separation volume shown here is based in part on experimental data, and in part on a geometric model of the shape of the separated region.

In Figure 4.3, the geometric model for the separation region is shown. This model consists of three parts: the upstream separation region, the roughness element itself, and the downstream separation region. The upstream separation volume, $V_{U S}$, is a half cone on its side with a base radius equal to the roughness height $k$, and a length to be determined. The roughness element volume, $V_{e}$, is known. The downstream separation volume, $V_{D S}$, is another half cone on its side of base radius $k$ and length to be determined. The total volume associated with a roughness element is then defined as

$$
V_{T}=V_{U S}+V_{e}+V_{D S}
$$

In order to determine the lengths of the half cone separation volumes, $h_{U S}$ and $h_{D S}$, LDV velocity profiles from the centerline were utilized. The streamwise location of a profile with respect to the roughness element provides a $x$-coordinate for a point on the separation surface. The point at which the streamwise velocity $U=0$ is assumed to be the $y$-location of a point on the separation surface. Given these streamwise and vertical coordinates, $h_{U S}$ 


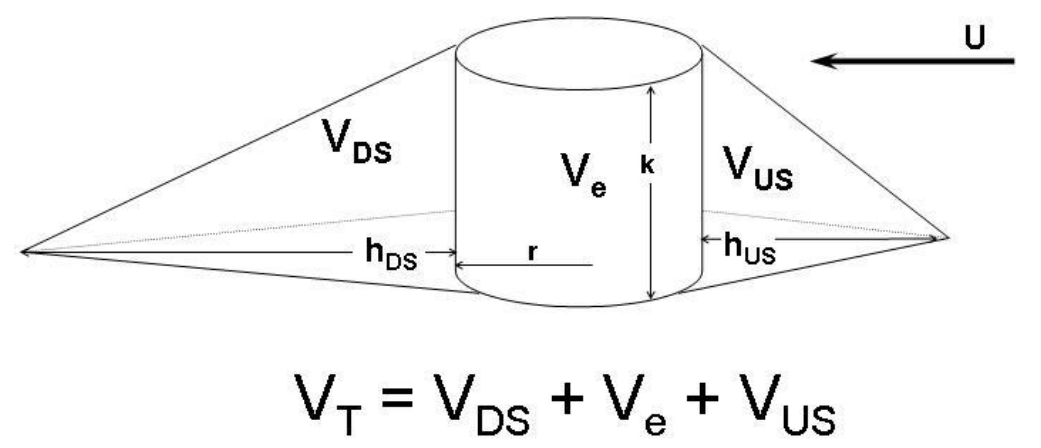

Figure 4.3: Geometric Model of Separation Volume

and $h_{D S}$ are determined by a linear fit to these points and the top of the roughness element for each of the four element geometries (Gaussian spike, $0.38 \mathrm{~mm}, 0.76 \mathrm{~mm}$, and $1.52 \mathrm{~mm}$ cylinders). The intersection of this line with the wall defines the half cone heights. The lines defined by the data for the downstream separation are shown in Figure 4.4.

The data used to find these locations on the separation surface is both that taken by this author, as well as that of George (2005). The separation area is assumed to be the same for a given element, regardless of its configuration, e.g. the volume for the Gaussian element in a staggered, $5.49 \mathrm{~mm}$ spaced configuration is the same as that for a Gaussian element in a straight, 8.23 $\mathrm{mm}$ in the vicinity of the roughness elements are of similar magnitude from one configuration to the next. This assumption also allowed using more data sets to help define the separation volume for each element. Spacing of the elements will be accounted for in a later step.

Very little data exist in the smaller, upstream separations. What data there are suggested that the height of the half cone defining the the upstream separation was approximately equal to the roughness height, $h_{D S} \approx k$. For the downstream separations, more data exists, and $h_{D S}$ is defined by the linear fit to the data, as shown in Figure 4.4.

From equation 4.6, the total volume associated with a single element is then equal to

$$
V_{T}=\frac{1}{6} \pi k^{3}+V_{e}+\frac{1}{6} \pi k^{2} h_{D S}
$$




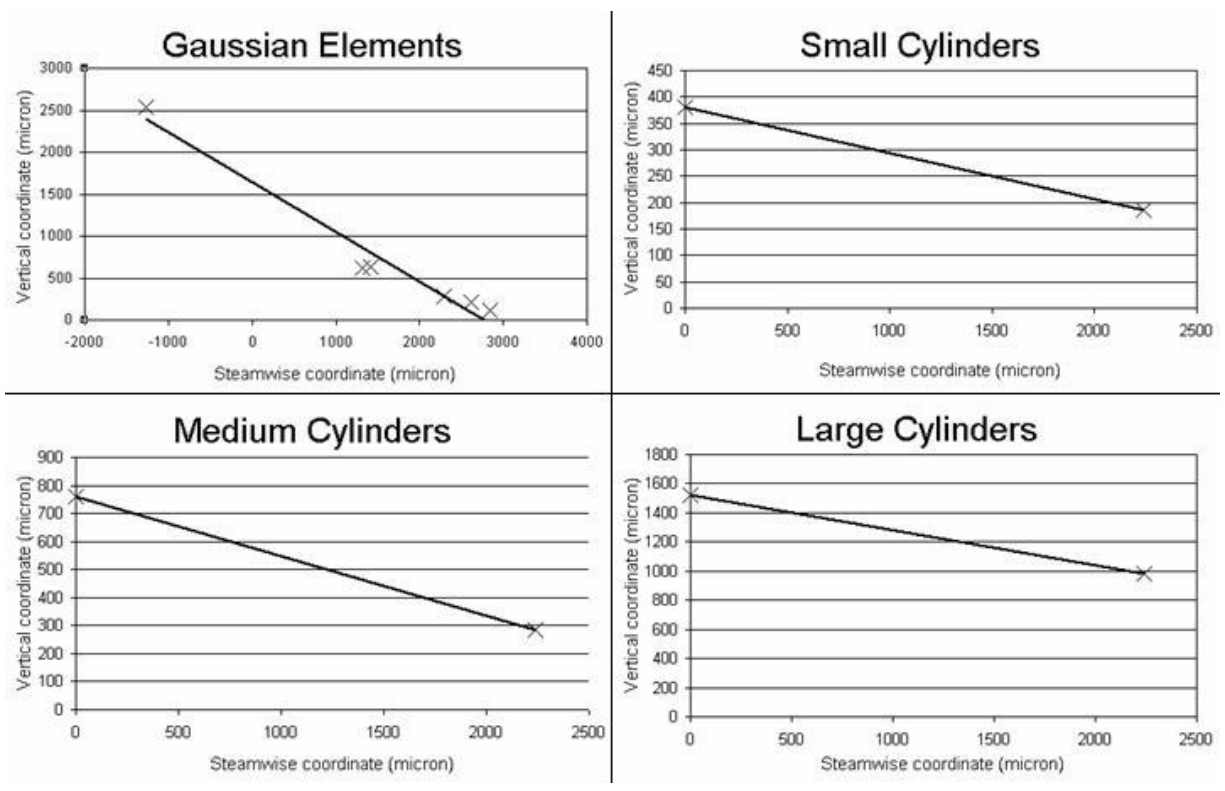

Figure 4.4: Determination of downstream separation volume with linear fit to experimental velocity data

For a surface covered in equally-spaced, identical roughness elements, the displacement height can be determined

$$
\epsilon=\frac{V_{T}}{D^{2}}
$$

Recall that $D$ is the element spacing.

The displacement heights calculated by both methods are included in Table 4.2. Other than a few exceptions (most notably the $5.49 \mathrm{~mm}$ spaced, straight configuration Gaussian spikes, case \#1) the geometric estimate of the displacement height closely matches that which satisfies Cole's Law of the Wall. To quantify the difference between the two calculation methods, the geometric method displacement height $\left(\epsilon_{\text {Geometric }}\right)$ is substituted into Equation 4.1 in lieu of the ideal curve fit value $\left(\epsilon_{\text {CurveFit }}\right)$, and the increase in the residual is is examined. That is, if the residual $R$ is defined as

$$
R=\overline{U_{\text {theory }}-U_{\text {exp }}}
$$

Where $U_{\text {theory }}$ is determined using Equation 4.1 from above, and $U_{\text {exp }}$ is the experimental data. $R$ is minimized when the curve fit value of displace- 


\begin{tabular}{|c|c|c|c|c|}
\hline Case \# & Configuration & $\epsilon_{\text {CurveFit }}^{+}$ & $\epsilon_{\text {Geometric }}^{+}$ & $R$ increase (\%) \\
\hline \hline 1 & Gaussian, 5.49 mm spacing, straight & 57.0 & 47.1 & $0.81^{*}$ \\
\hline 2 & Gaussian, 5.49 mm spacing, staggered & 47.0 & 49.7 & 0.09 \\
\hline 3 & Gaussian, 8.23 mm spacing, straight & 19.3 & 20.5 & 0.50 \\
\hline 4 & Gaussian, 8.23 mm spacing, staggered & 24.0 & 18.9 & 3.92 \\
\hline 5 & $0.38 \mathrm{~mm}$ cylinder, straight & 2.2 & 2.6 & 0.27 \\
\hline 6 & $0.38 \mathrm{~mm}$ cylinder, staggered & 2.4 & 2.6 & 0.09 \\
\hline 7 & $0.76 \mathrm{~mm}$ cylinder, straight & 3.9 & 6.0 & 3.20 \\
\hline 8 & $0.76 \mathrm{~mm}$ cylinder, staggered & 5.3 & 6.3 & 1.53 \\
\hline 9 & $1.52 \mathrm{~mm}$ cylinder, straight & 22.0 & 18.0 & 1.11 \\
\hline 10 & $1.52 \mathrm{~mm}$ cylinder, staggered & 18.3 & 17.2 & 0.64 \\
\hline
\end{tabular}

Table 4.2: Normalized displacement height as calculated by fit to Cole's Law of the Wall and by geometric method

ment height, $\epsilon_{\text {CurveFit }}$, is used. The percent increase in the residual $R$ when $\epsilon_{\text {Geometric }}$ is used instead indicates how well the geometric method of determining displacement height matches the experimental data.

This increase in $R$ is typically less than $2 \%$. In the case of the 5.49 mm spaced, straight Gaussian spikes (Case \#1), the error is less than $1 \%$, but only if the roughness effect $\Delta u^{+}$is also adjusted slightly in the curve fit (from 12.4 to 12.5 ). This case is denoted with an asterisk in Table 4.2. The residual increase in the $8.23 \mathrm{~mm}$ spaced, staggered Gaussian spikes (Case \#4) and the $0.76 \mathrm{~mm}$ straight cylinders (Case \#7) is under $4 \%$, moderately greater than that of the other cases.

In order to further test the effectiveness of the geometric method of determining the displacement height, $\epsilon_{\text {Geometric }}$ is calculated for the six roughness cases of George (2005). Here, $V_{T}$ is determined based on cross-sectional ( $z$ plane) areas of the mean separation volume provided by George, and $\epsilon_{\text {Geometric }}$ is calculated by Equation 4.8. These values are then compared with the displacement height values given by George, which are based on a fit to Cole's Law of the Wall. These results can be seen in Table 4.3. Four of the six cases appear to agree, though the two values for displacement height differ significantly for the cases of the staggered $1.52 \mathrm{~mm}$ and $0.76 \mathrm{~mm}$ cylinders. Typically, displacement height values calculated by either method do not vary much between a straight and staggered configuration. It is not clear 


\begin{tabular}{|c|c|c|}
\hline Configuration & $\epsilon_{\text {CurveFit }}^{+}$ & $\epsilon_{\text {Geometric }}^{+}$ \\
\hline \hline $0.38 \mathrm{~mm}$ cylinder, straight & 14 & 13.1 \\
\hline $0.38 \mathrm{~mm}$ cylinder, staggered & 11 & 12.4 \\
\hline $0.76 \mathrm{~mm}$ cylinder, straight & 27 & 29.0 \\
\hline $0.76 \mathrm{~mm}$ cylinder, staggered & 19 & 28.7 \\
\hline $1.52 \mathrm{~mm}$ cylinder, straight & 62 & 61.4 \\
\hline $1.52 \mathrm{~mm}$ cylinder, staggered & 44 & 62.5 \\
\hline
\end{tabular}

Table 4.3: Normalized displacement height as calculated by the geometric method, compared with curve fit values of George (2005). Note that the element spacing for the George (2005) cases $(5.49 \mathrm{~mm})$ is half of the spacing in the cylindrical roughness data of this author $(10.97 \mathrm{~mm})$.

why this is the case for the data of George (2005). More data from these cases may be required to resolve the discrepancies.

It has been seen that the displacement height predicted by the geometric method fairly closely predicts the displacement height calculated by the semilogarithmic law of the wall. This indicates that the reasoning behind the geometric method is sound, and the model of the separation volumes is sufficiently accurate. However, the main drawback to the method is that mean velocity measurements must be taken in the vicinity of the roughness element to help define the separation volume, and this is not a trivial exercise.

\subsection{Wake Parameter}

The wake parameter, $\Pi$, was first discussed in Section 1.5. Some roughness studies, such as the review by Tani (1987), have shown a large variation in the value for $\Pi$. The smooth wall zero pressure gradient value is approximately 0.52 (Fernholz and Finley, 1996), and rough wall values have been found to range up to 1 . Jimenez (2004) proposed that this is a function of the roughness height to boundary layer thickness ratio, $k / \delta$, and the data here tend to support that idea.

The $k / \delta$ is essentially a ratio of the length scales of the vortices present in a rough wall boundary layer. The largest vortices present are of a size on the 
order of the boundary layer thickness, $\delta$. The inner layer vortices are those produced by the roughness elements themselves, and thus are of the scale of the roughness height, $k$.

There is an analogy that can be made to a smooth wall turbulent boundary layer. In a smooth wall flow, once again the largest vortices in the boundary layer are of the order $\delta$. The inner layer vortices, however, are those produced on the wall, and are of scale of the thickness of the viscous sublayer, or $y^{+} \approx 10$. Thus, the ratio of these scales could be represented as

$$
\frac{y^{+}=10}{\delta}=\frac{\frac{10 \nu}{u_{\tau}}}{\delta}=\frac{10 \nu}{\delta u_{\tau}}
$$

Both this smooth wall ratio and the rough wall ratio $k / \delta$ indicate the level of interaction between the inner and outer layers of a boundary layer. For small ratios, such as those typically found in a smooth wall and for rough walls with small elements, the near wall vortices have little impact on the outer layer flow. The effect of the wall is apparent to the outer layer only in the value of the friction velocity $u_{\tau}$. However, as this scale ratio increases, the inner layer vortices are significant, and have a direct effect in the outer layer. In the present study, a fairly wide range of $k / \delta$ is covered, as seen in Table 4.1 .

The values of the wake parameter $\Pi$, as determined by fitting Equation 4.1, are shown in Table 4.1. They vary greatly, from lower values, associated with smaller $k / \delta$, to values slightly above 1 . The variation of $\Pi$ with scale ratio $k / \delta$ is more clearly visible in Figure 4.5. A linear trendline has been added to the Figure to emphasize the trend, but it has no physical basis. Figure 4.5 also shows the results of George (2005), also determined by a fit

of velocity profile data to Equation 4.1. These data follow the same trend as that of the present study.

\subsection{Roughness Correlations}

The concept of correlating the roughness effect $\Delta U / u_{\tau}$ with the geometry and spacing of the roughness elements was first introduced in Section 1.4. Dvorak (1969) presented a correlation for wide variety of data, including 


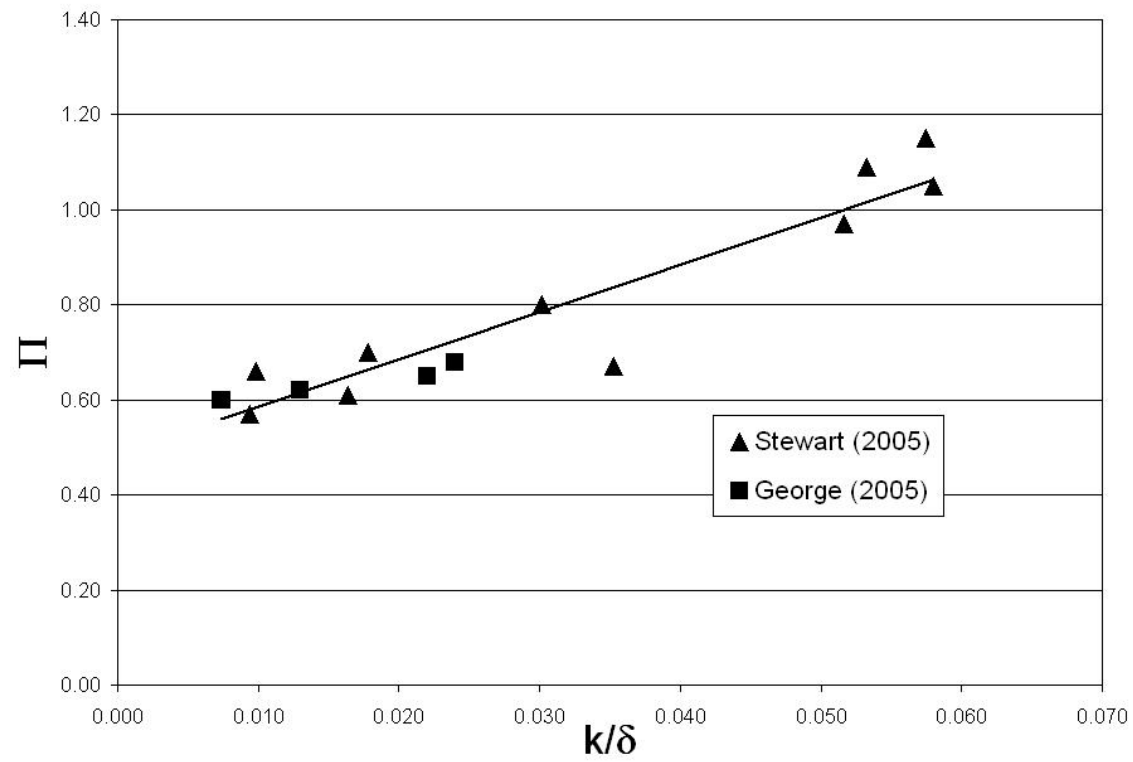

Figure 4.5: Plot of wake parameter $\Pi$ versus scale ratio $k / \delta$.

the two-dimensional rods of Betterman (1966), the sand grain roughness of Nikuradse (1933), and the spheres of Schlichting (1936), among others. The roughness length scales defined a parameter $\lambda_{D}$, which Dvorak defined as the ratio of the surface area covered by the roughness elements to the total surface area. The $\lambda$ was then correlated to $f(\lambda)$, which was a function of the roughness effect $\Delta U / u_{\tau}$

$$
f(\lambda)=\frac{\Delta U}{u_{\tau}}-\frac{1}{\kappa} \ln \left(\frac{k u_{\tau}}{\nu}\right)
$$

Dvorak proposed two correlations, one for sparsely distributed roughness elements (large $1 / \lambda_{D}$ ), and another for more closely packed roughness elements (small $\left.1 / \lambda_{D}\right)$. For $1 / \lambda_{D} \geq 4.68$, Dvorak proposed that

$$
f(\lambda)=-5.95\left(1.103 \log \left(1 / \lambda_{D}\right)-1\right)
$$

For $\lambda \leq 4.68$, Dvorak's correlation was

$$
f(\lambda)=17.35\left(1.625 \log \left(1 / \lambda_{D}\right)-1\right)
$$




\begin{tabular}{|c|c|c|c|}
\hline Case \# & Configuration & $\Delta U / u_{\tau}$ & $f(\lambda)$ \\
\hline \hline 1 & Gaussian, 5.49 mm spacing, straight & 12.4 & -1.07 \\
\hline 2 & Gaussian, 5.49 mm spacing, staggered & 12.7 & -0.90 \\
\hline 3 & Gaussian, 8.23 mm spacing, straight & 11.1 & -2.32 \\
\hline 4 & Gaussian, 8.23 mm spacing, staggered & 9.7 & -3.51 \\
\hline 5 & $0.38 \mathrm{~mm}$ cylinder, straight & 2.3 & -5.64 \\
\hline 6 & $0.38 \mathrm{~mm}$ cylinder, staggered & 1.9 & -5.96 \\
\hline 7 & $0.76 \mathrm{~mm}$ cylinder, straight & 5.1 & -4.73 \\
\hline 8 & $0.76 \mathrm{~mm}$ cylinder, staggered & 5.7 & -4.27 \\
\hline 9 & $1.52 \mathrm{~mm}$ cylinder, straight & 7.9 & -4.00 \\
\hline 10 & $1.52 \mathrm{~mm}$ cylinder, staggered & 7.4 & -4.38 \\
\hline
\end{tabular}

Table 4.4: Roughness effect $\Delta U / u_{\tau}$ and $f(\lambda)$

Simpson (1973) found better agreement between experimental data and these correlations if a different definition of $\lambda$ is used. Simpson proposed that

$$
1 / \lambda=\frac{A_{S}}{A_{F}}
$$

where $A_{S}$ is the total wall surface area and $A_{F}$ is the frontal area of the elements normal to the flow. The use of frontal area to determine $1 / \lambda$ gives a better description of the roughness element geometry and its effect on the flow. Lines representing the correlations of Dvorak (1969) with the $1 / \lambda$ of Simpson (1973) appear in Figures 4.6 and 4.9.

For the data taken in the present study, the values of the roughness effect $\Delta U / u_{\tau}$, as determined by the fit to Equation 4.1 , and calculated values of $f(\lambda)$ appear in Table 4.4. The $f(\lambda)$ is plotted with $1 / \lambda$ in Figure 4.6. Included in this figure is the data of George (2005), and the correlation of Dvorak (1969). Generally, the data agree well with the correlation. The exception are the cases with the largest values of $1 / \lambda$, corresponding to the cylindrical elements of $0.38 \mathrm{~mm}$ and $0.76 \mathrm{~mm}$ heights.

The most likely reason that $f(\lambda)$ for the $0.38 \mathrm{~mm}$ and $0.76 \mathrm{~mm}$ cylindrical cases from the present study are greater than what is predicted by the correlation is that they are in the transitional regime. Simpson (1973) notes that this correlation is for the completely rough regime. Dvorak (1969) extended the correlation to the transitional regime, but this was based on the 


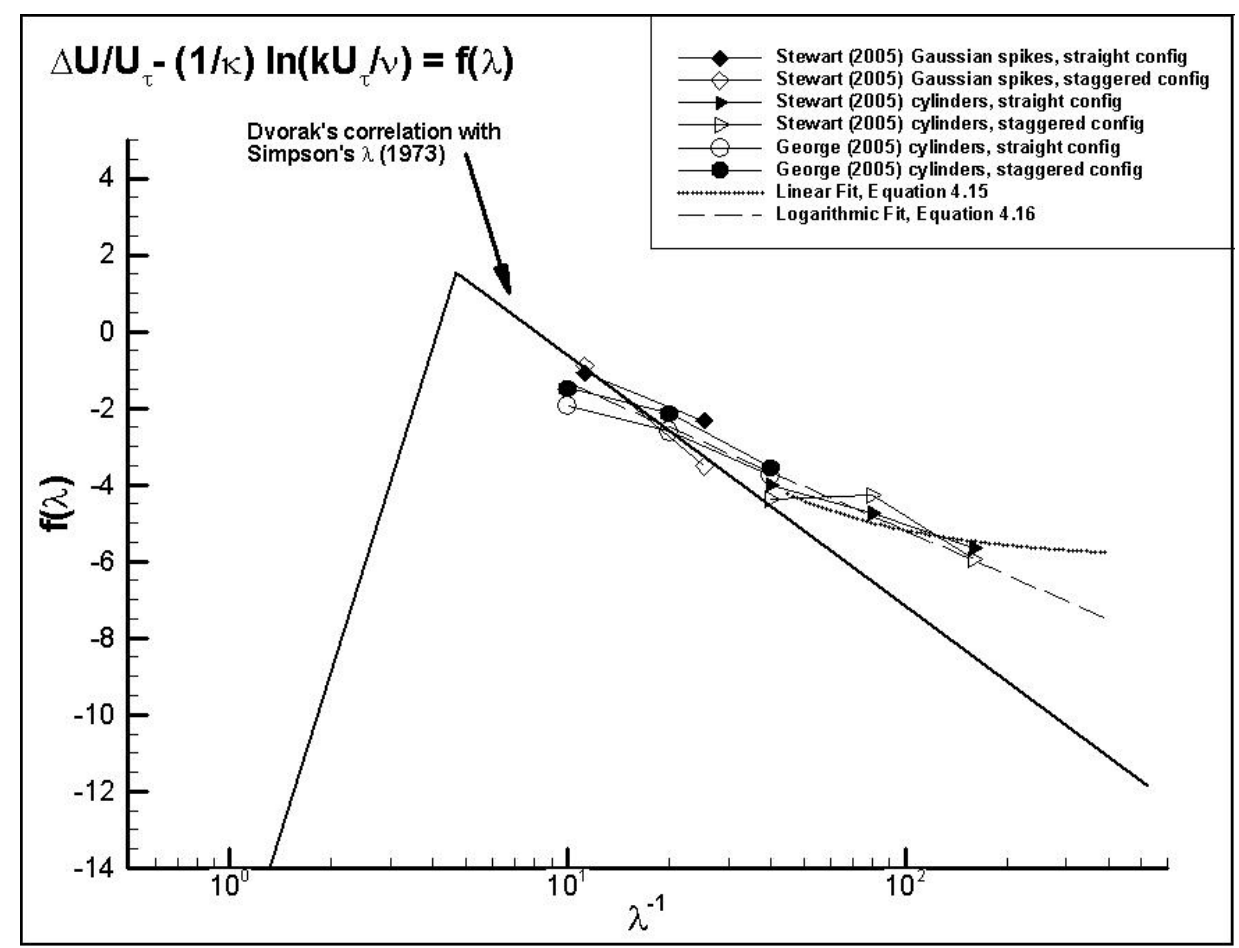

Figure 4.6: Plot of $f(\lambda)$ vs. $\lambda^{-1}$. 
sand grain roughness of Nikuradse (1933) and the screens of Hama (1955). These types of roughness behave significantly different than the distributed, three-dimensional roughness elements being considered here.

In the completely rough regime, force acting tangentially to the surface is assumed to be due to the drag on the roughness elements, rather than any viscous drag on the wall itself. The separation regions and the roughness elements themselves cover most of the surface area of the wall. However, as the element spacing becomes wider and/or the elements become smaller (thus creating smaller separation regions), more smooth wall area is exposed to attached flow. Thus, skin friction drag on the wall becomes increasingly important in addition to the form drag on the elements.

This phenomenon would account for the results seen in Figure 4.6. In the cases of the relatively large Gaussian elements and $1.52 \mathrm{~mm}$ cylinders (along with all of the more closely-spaced cylinders of George (2005)), the skin friction drag is negligible. However, for the $0.38 \mathrm{~mm}$ and $0.76 \mathrm{~mm}$ cylinders with a $10.97 \mathrm{~mm}$ spacing, the $f(\lambda)$ is due to both skin friction from the wall and form drag from the elements. The result is a value of $f(\lambda)$ higher than predicted by the correlation, which typically only applies in the completely rough regime.

Figure 4.6 shows a possible relationship between $\lambda$ and $f(\lambda)$ for the experimental data in the transitional region. It is a linear function, obtained by a fit to the experimental data points for the transitional roughness. For the region $\lambda^{-1}>40$

$$
f(\lambda)=77.10 \lambda-5.96
$$

Note that the dependent variable in Equation 4.15 is $\lambda$, not $1 / \lambda$.

This relationship implies a linear, steady increase in the transitional effects on $f(\lambda)$ as roughness density $\lambda$ increases. This is analogous to the linear relationship between $\Delta U / u_{\tau}$ and $k^{+}$in the transitional region described by Dvorak (1969).

Alternatively, the present data, along with that of George (2005), follow a log-linear trend defined by

$$
f(\lambda)=-1.673 \ln \left(\lambda^{-1}\right)+2.516
$$

This line is also shown in Figure 4.6. The data fit this line with an $R^{2}$ of 0.926. However as will be seen in later figures, no other data sets follow 
the trends in Equations 4.15 and 4.16, so it is premature to try to use these relationships to predict other rough wall flow behavior.

The disagreement between the experimentally obtained values for $f(\lambda)$ and the correlation of Dvorak (1969) (with Simpson's $\lambda$ ) has been attributed to the significant effect of skin friction drag on the portions of the wall exposed to attached flow. However, this conclusion, as well as Equation 4.15, is clearly based on very limited experimental data, and further study is required to determine its validity.

A functional relationship between $\lambda$ and experimental $f(\lambda)$ values was found using a model equation similar to the Spalding equation. This equation blends the linear relationship in the transitional region (Equation 4.15) and blends it with the logarithmic relationship in the fully rough region.

$k^{+}+S=\Delta U^{+}+\frac{1}{\exp (\kappa f(\lambda))}\left\{\exp \left(\kappa \Delta U^{+}\right)-\left[1+\kappa \Delta U^{+}+\frac{\left(\kappa \Delta U^{+}\right)^{2}}{2 !}+\frac{\left(\kappa \Delta U^{+}\right)^{3}}{3 !}\right]\right\}$

Where $S$ is a adjusted to match the data. Equation 4.17 can be arranged to form an explicit equation for $f(\lambda)$.

$$
f(\lambda)=\frac{1}{\kappa} \ln \left[\frac{\left\{\exp \left(\kappa \Delta U^{+}\right)-\left[1+\kappa \Delta U^{+}+\frac{\left(\kappa \Delta U^{+}\right)^{2}}{2 !}+\frac{\left(\kappa \Delta U^{+}\right)^{3}}{3 !}\right]\right\}}{k^{+}+S-\Delta U^{+}}\right]
$$

Using a value of 46 for $S$, Equation 4.18 is plotted in Figure 4.8.

Values of $U^{+}$are plotted versus $k+$ in Figure 4.7. Experimental data are shown, along with corresponding values calculated via Equation 4.17. Lines of constant $\lambda$ are shown, with slopes estimated from the experimental data, along with the data of George (2005) and Dvorak (1969).

Figure 4.9 is similar to Figure 4.6 above, but adds some additional data sets. Data from O'Loughlin (1965), Koloseus and Davidian (1966), Raupach et al. (1980), and Tomkins and Adrian (2000) have been included. It can be seen that there is a collapse of these data sets to the Dvorak correlation from the range of $8 \geq \lambda^{-1} \geq 50$. However, in the range of $\lambda^{-1}>50$, there is significant divergence of the data sets.

The data on the cube roughness of O'Loughlin (1965) are mostly below the range of $\lambda^{-1}$ that is being discussed here. However, the data at $\lambda^{-1}=8.8$ 


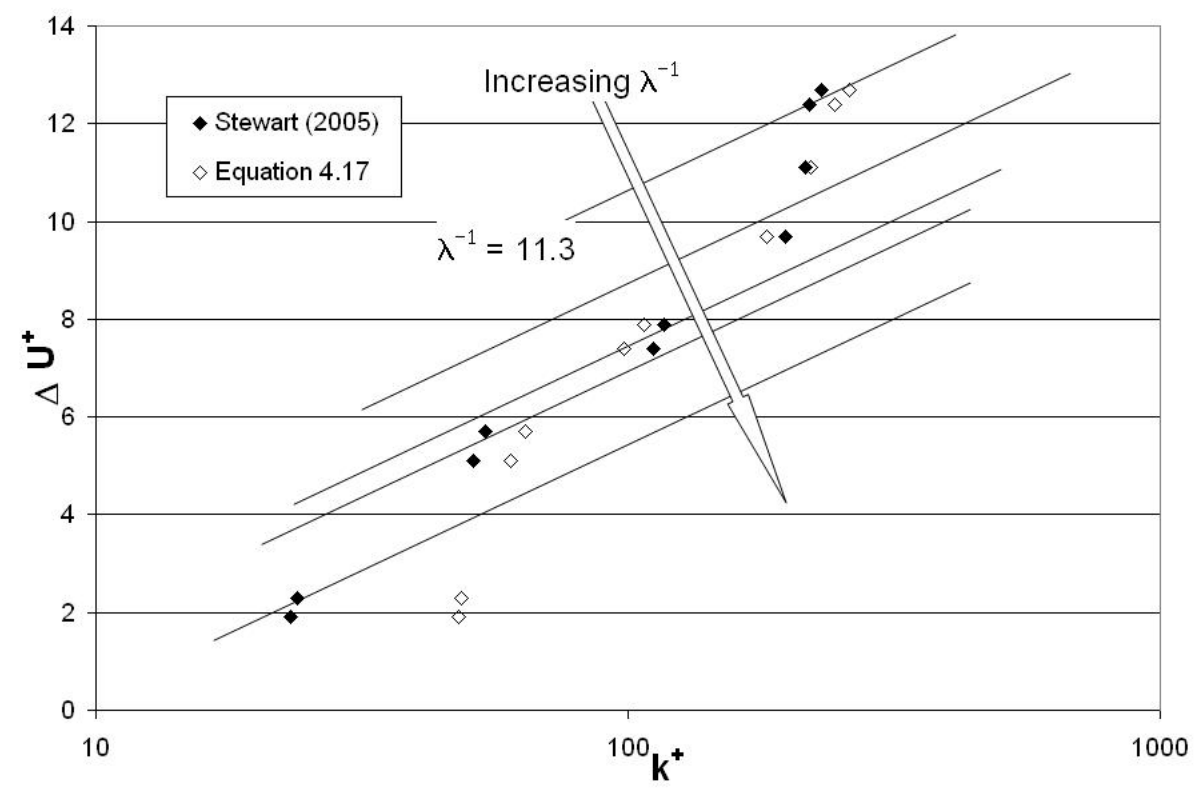

Figure 4.7: Plot of $U^{+}$versus $k+$ with estimated lines of constant $\lambda$.

have collapsed with the Dvorak correlation.

Koloseus and Davidian (1966) obtained data on a channel flow roughened with $4.76 \mathrm{~mm}$ high cubes over a large range of $\lambda^{-1}$. In the lower range, the data agrees well with the correlation, but diverges in the range $\lambda^{-1}>100$.

Data were obtained by Raupach et al. (1980) on circular cylinders over a large range of $\lambda^{-1}$. All of the values of $f(\lambda)$ agree well with the correlation for the range $\lambda^{-1}>10$, including a point at a $\lambda^{-1}=89$.

Tomkins and Adrian (2000) obtained particle image velocimetry (PIV) data on two sizes of hemispheres. The data on the larger spheres are above the Dvorak correlation, but not significantly so, and it agrees well with the Gaussian spike data taken in this study. The data corresponding to the smaller hemispheres are below the value predicted by the Dvorak correlation.

The data included in Figure 4.9 do not show a collapse, either with the Dvorak correlation or with a different trend, in the range of $\lambda^{-1}>50$. The lack of a clear trend is due in part to the low number of data sets available in 


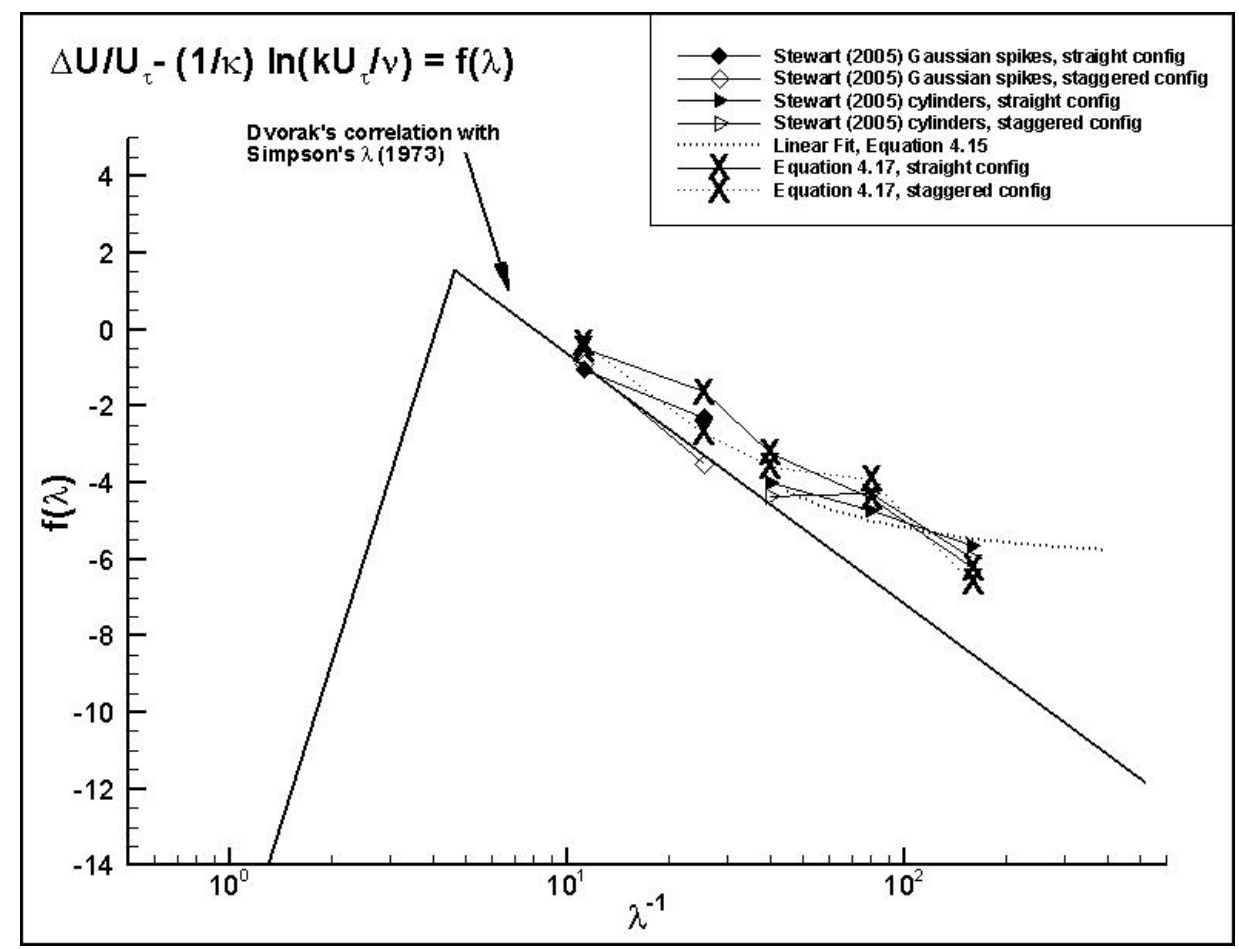

Figure 4.8: Plot of $f(\lambda)$ vs. $\lambda^{-1}$, with functional relationship defined in Equation 4.18. 


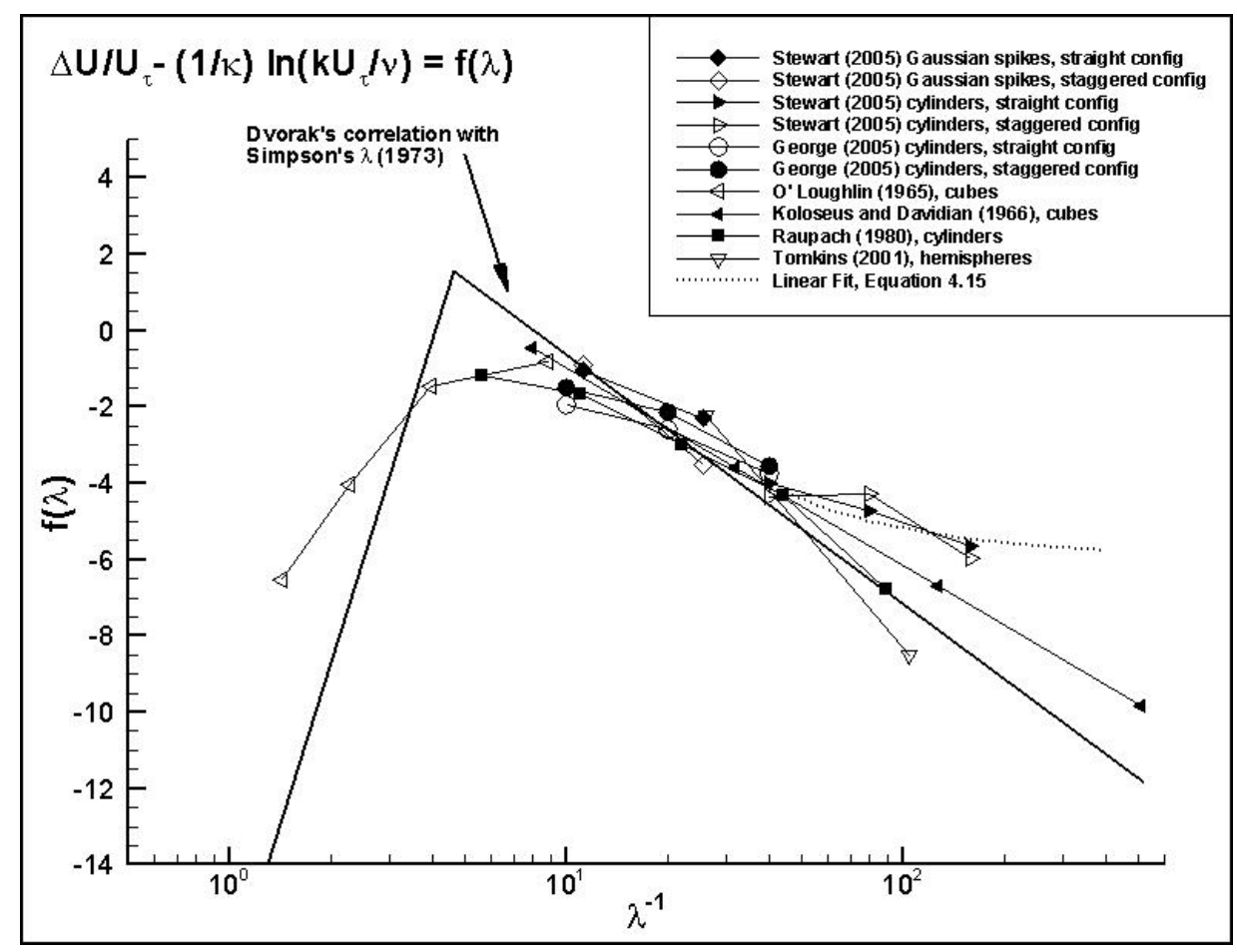

Figure 4.9: Plot of $f(\lambda)$ vs. $\lambda^{-1}$, with data of additional authors. 
this region. However, it can be noted that the data of Koloseus and Davidian (1966) and Tomkins and Adrian (2000) (and clearly that of Raupach et al. (1980)) agree better with the Dvorak correlation than the data obtained in this study. A possible reason is that the $k^{+}$values for the $0.76 \mathrm{~mm}$ and $0.38 \mathrm{~mm}$ cylinders in this study are much smaller than those in the above mentioned studies. The $0.76 \mathrm{~mm}$ cylinders have a $k^{+}$of approximately 50 , while the $0.38 \mathrm{~mm}$ cylinders have a $k^{+}$of approximately 24 . By contrast, the cylinders of Raupach et al. (1980) have $k^{+} \approx 300$, the cubes of Koloseus and Davidian (1966) have $k^{+} \approx 300-350$, and the smaller hemispheres of Tomkins and Adrian (2000) have $k^{+} \approx 100$.

By the previous reasoning, the over-prediction of $f(\lambda)$ was due to the increased significance of skin friction drag for the smaller cylindrical elements. This is supported by the values of $k^{+}$. For a rough wall with larger values of $k^{+}$, the separation areas on the elements will be larger, and form drag will be more significant. Though some transitional effects may exist in the data of Koloseus and Davidian (1966), Raupach et al. (1980), and Tomkins and Adrian (2000), and thus may diverge somewhat from the Dvorak correlation, they are still not as significant as in the data from this study, which have comparatively very low values of $k^{+}$. It remains to be seen in what manner $\lambda^{-1}, k^{+}$, and other parameters can be used to consistently predict the effects of transitional roughness on $f(\lambda)$. Further data, taken over a range of $k^{+}$ values at $\lambda^{-1}>50$ is required in order to systematically define these effects. 


\section{Chapter 5}

\section{Conclusions \& Future Work}

\subsection{Summary of Study}

An experimental study has been conducted of the effects of three-dimensional distributed roughness elements on a two-dimensional turbulent boundary layer. This study was conducted in the Virginia Tech Small Boundary Layer Wind Tunnel.

Two types of roughness were considered: circular cylindrical posts and Gaussian spikes. Three heights of cylinders were considered, $0.38 \mathrm{~mm}, 0.76$ $\mathrm{mm}$, and $1.52 \mathrm{~mm}$, all with a diameter of $1.98 \mathrm{~mm}$ and a spacing of $10.97 \mathrm{~mm}$. The Gaussian spikes studied were $2.54 \mathrm{~mm}$ in height, and had a base diameter of $2.54 \mathrm{~mm}$. Two spacings of Gaussian element were considered, $5.49 \mathrm{~mm}$ and $8.23 \mathrm{~mm}$. All of these geometries were studied in both a "straight" and "staggered" configuration, yielding a total of ten cases. Note that data was acquired for the Gaussian roughness with $5.49 \mathrm{~mm}$ spacing in the straight configuration by Bennington (2004), but was included in the analysis for this study.

Measurements were taken using a three-dimensional fiber optic Laser Doppler Velocimeter (LDV). This system has a fine-resolution $(\approx 50 \mu \mathrm{m})$ measurement volume. For each roughness configuration, three vertical measurement profiles were taken in the centerline plane downstream of a roughness element, along with four additional profiles in the mid-plane between 
two rows of elements. An additional profile was taken 6-8 cells downstream of the primary measurement location. Mean velocities, Reynolds stresses, and triple products were obtained for each profile. A single-component Outer Layer LDV system was used to take measurements of mean streamwise velocity in the in the region of the boundary layer beyond the reach of the three-component system.

\subsection{Conclusions}

Flow around the roughness element is characterized by high speed fluid coming into the separation region behind the element both from the gaps between elements, and rushing toward the wall from above the element. Sweeping motions bring high-momentum fluid toward the wall, while an ejection of lowmomentum fluid occurs in small region immediately behind the roughness element.

The mean $U$-velocity profiles show the presence of the backflow region behind the roughness elements, and exhibit a semi-logarithmic profile which has a shift $\Delta U / u_{\tau}$ based on the roughness density. This semi-logarithmic region exists in all of the measured cases, but is weaker in cases with a larger $k / \delta$.

The interface between the low-momentum fluid below the roughness height and the higher-momentum fluid above creates a strong mixing region. This is evidenced by high levels of turbulent kinetic energy near the element height. $\overline{v^{2}} / u_{\tau}^{2}, \overline{w^{2}} / u_{\tau}^{2}$, and $\overline{u v} / u_{\tau}^{2}$ stresses also exhibit peaks near the element height much greater than levels seen in a smooth wall profile. $\overline{u^{2}} / u_{\tau}^{2}$ normal stress, by contrast, is lower than that of a smooth wall boundary layer.

Though the general behavior of the flow around the Gaussian elements is similar to that around the cylindrical elements, significant differences do exist. The flat tops of the cylindrical elements produce a sharp interface between the low-speed near-wall fluid, and the high speed fluid above. The result of this are well-defined, high peaks in turbulent stresses. The pointed tops of the Gaussian elements do not create this sharp interface, and mixing occurs over a range of heights. Profiles of turbulent stresses taken on Gaussian roughness fetches exhibit broad (even multiple) peaks, showing tur- 
bulence not just near the roughness height, but significantly below it. However, the stresses never reach the magnitude at any one point the stresses on the cylindrical fetches do. Turbulent mixing is more spread out in the near wall region for the Gaussian spike roughness than for the cylindrical elements.

Data on cylindrical elements as well as Gaussian elements shows that for a given roughness element geometry, the maximum magnitude of turbulent quantities tends to increase as element spacing increases.

The effect of the roughness on the boundary layer mean velocity profile was quantified by fitting Cole's Law of the Wake and semilogarithmic law of the wall to the experimental data. The variables used in this fit were the displacement height, $\epsilon$, the roughness effect, $\Delta U / u_{\tau}$, and the wake parameter, $\Pi$. Trends in each of these three parameters were examined.

The displacement height $\epsilon$ is the location of the effective origin above the actual wall. Besides being determined by the law of the wall, this parameter was also calculated by estimating the volume of the element and separation region. Separation volume was estimated using LDV data and a geometric model for the shape of the separation. This method agreed well with the curve fit value, causing less than a $4 \%$ increase in the residual when used in the curve fit model equation.

The values for the wake parameter $\Pi$ were found to vary widely for the various roughness configurations, from 0.6 to 1.1 , which is significantly greater than typical smooth wall values of approximately 0.52 (Fernholz and Finley, 1996). The values for $\Pi$ increase with increasing ratios of roughness height to boundary layer thickness, $k / \delta$. The values from the present study, along with those of George (2005), follow a linear trend.

The main effect of the roughness on the velocity profile is to shift the semi-logarithmic region by a quantity $\Delta U / u_{\tau}$. Once determined, this shift was used to calculate $f(\lambda)=\Delta U / u_{\tau}-(1 / \kappa) \ln \left(k u_{\tau} / \nu\right)$. This value of $f(\lambda)$ was compared to that predicted by the correlation of Dvorak (1969), using the roughness density $\lambda^{-1}$ defined by Simpson (1973). It was found that the cases with lower values of $\lambda^{-1}$, which included the four Gaussian spikes and the two $1.52 \mathrm{~mm}$ cylinder cases, agree well with this correlation. However, the four cases with the smaller cylinders $(0.38$ and $0.76 \mathrm{~mm})$ had calculated 
values of $f(\lambda)$ significantly greater than that predicted by the correlation. This was attributed to the fact that in addition to the form drag on the elements, significant skin friction drag was occurring due to the exposed smooth walls. The cumulative effect of form and skin friction drag was a higher value of $f(\lambda)$. It was proposed that these data follow a linear trend, similar to the linear relationship between $\Delta U / u_{\tau}$ and $k^{+}$in the transitional region described by Dvorak (1969). The data from the present study, along

with data from other authors, tends to confirm the Dvorak correlation in the range $8 \geq \lambda^{-1} \geq 50$, but the current data does not support the correlation above that range.

\subsection{Future Work}

Continued research will focus on more densely packed roughness elements, and their effect on a turbulent boundary layer. Using the ability of Laser Doppler Velocimetry to measure on a fine scale, measurements will be taken around closely packed elements. Among other things, this data will serve to determine trends in the roughness effect $\Delta U / u_{\tau}$ in ranges below $\lambda^{-1}=5$.

Currently few studies have obtained data on transitional roughness, as it is typically more difficult to analyze than that acquired in fully rough flows. It is recommended that further research be conducted in this area in order to examine the behavior of the roughness effect for sparsely distributed roughness, not only over a range of $\lambda^{-1}$, but also $k^{+}$. This study would serve to explain the coupled effects of skin friction and form drag on the structure of the turbulent boundary layer. 


\section{Bibliography}

Lodewijk Helena Jozef Absil. Analysis of the Laser Doppler Measurement Technique for Application in Turbulent Flows. PhD thesis, Technische Universiteit Delft, 1995.

A.J. Adams, D.E. Wennerstrom, and M.K. Mazumder. Use of bacteria as model nonspherical aerosol particles. Journal of Aerosol Science, 16:337343, 1985 .

T. Alfrey, E.B. Bradford, and J.W. Vanderhoff. Journal of the Optical Society of America, 44:603-609, 1954.

D. Baker. A report on the vaporization/condensation aerosol generator built at Virginia Tech. Internal Report, September 1986.

J.L. Bennington. Effect of various shaped roughness elements in twodimensional high reynolds number turbulent boundary layers. Master's thesis, Virginia Tech, 2004.

D. Betterman. Contribution à l'etude de la couche limite turbulente le long de plaques rugueuses. International Journal of Heat and Mass Transfer, 9:153-164, 1966.

P. Bradshaw. A note on "critical roughness height" and "transitional roughness". Physics of Fluids, 12:1611-1614, 2000.

H. Chang and K. Okuyama. Optical properties of dense and porous spheroids consisting of primary silica nanoparticles. Journal of Aerosol Science, 33: 1701-1720, 2002.

C.K. Chen and J.A. Roberson. Turbulence in wakes of roughness elements. ASCE Journal of the Hydraulics Division, 100:53-67, 1974. 
C.J. Chesnakas. The Miniature, 3-D, Fiber-Optic, Laser Doppler Velocimeter. Internal Report, Virginia Tech Aerospace \& Ocean Engineering Department, 1995.

C.F. Colebrook. Turbulent flow in pipes, with particular reference to the transition region between smooth and rough pipe laws. Journal of the Institute of Civil Engineers, 11:133-156, 1939.

H.W. Coleman, B.K. Hodge, and R.P. Taylor. A re-evaluation of Schlichting's surface roughness experiment. Journal of Fluids Engineering, 106:60-65, 1984.

D.E. Coles. The law of the wake in the turbulent boundary layer. Journal of Fluid Mechanics, 1:191-226, 1956.

H. Darcy. Recherches expérimentales relatives au mouvement de l'eau dans les tuyaux. Mallet-Bachelier, 1857.

R.B. Dirling, Jr. A method for computing roughwall heat transfer rates on reentry nosetips. AIAA Paper 73-763, 1973.

F. Durst, A. Melling, and J.H. Whitelaw. Principles and Practice of Laser Doppler Anemometry. Academic Press, second edition, 1981.

F. Durst, R. Martinuzzi, J. Sender, and D. Thevenin. LDV-measurements of mean velocity, RMS-values and higher order moments of turbulence intensity fluctuations in flow fields with strong velocity gradients. In Proceedings of the 6th International Symposium on Applications of Laser Techniques to Fluid Mechanics, Lisbon, Portugal, 1992. paper 5.1.1.

F. Durst, J. Jovanovic, and J. Sender. LDA measurements in the near-wall region of a turbulent pipe flow. Journal of Fluid Mechanics, 295:305-335, 1995.

F.A. Dvorak. Calculation of turbulent boundary layers on rough surfaces in pressure gradient. AIAA Journal, 7:1752-1759, 1969.

H.H. Fernholz and P.J. Finley. The incompressible zero-pressure-gradient turbulent boundary layer: an assessment of the data. Progress in Aerospace Science, 32:245-311, 1996. 
N.A. Fuchs and A.G. Sutugin. Generation and use of monodisperse aerosols. In C.N. Davies, editor, Aerosol Science, pages 1-30. Academic Press, London, 1966.

G.E. Gajnos. Heat systems - Ultrasonics, Inc. nozzle testing. Technical Report CFH Report No. 800821, CFH Research Laboratories, Springfield, MA.

J. George. Structure of 2-D and 3-D Rough-Wall Turbulent Boundary Layers with Sparsely Distributed Roughness Elements. PhD thesis, Virginia Tech, 2005.

J. George and R.L. Simpson. Transport-rate budgets of reynolds stresses in 2-d and 3-d rough-wall turbulent boundary layers. AIAA Paper 2004-1286, 2004.

J. George and R.L. Simpson. Some effects of sparsely distributed threedimensional roughness elements on two-dimensional turbulent boundary layers. AIAA Paper 2000-0915, 2000.

G. Hagen. Über den einfluss der temperatur auf die bewegung des wassers in röhren. Math. Abh. Akad. Wiss., pages 17-98, 1854.

F.R. Hama. Boundary-layer characteristics for smooth and rough surfaces. American Society of Naval Architects and Marine Engineers, 62:260-270, 1955.

P.S. Jackson. On the displacement height in the logarithmic velocity profile. Journal of Fluid Mechanics, 111:15-25, 1981.

J. Jimenez. Turbulent flows over rough walls. Annual Review of Fluid Mechanics, 36:173-196, 2004.

H.J. Koloseus and J. Davidian. Roughness concentration effects on flow over hydrodynamically rough surfaces. USGS Water Supply Paper 1592D, 1966.

H.J. Koloseus, R.W. Powell, and D.F. Peterson, Jr. Roughness spacing in rigid open channels. ASCE Journal of the Hydraulics Division, 88:97-107, 1962. 
P.A. Krogstad and R.A. Antonia. Surface roughness effects in turbulent boundary layers. Experiments in Fluids, 27:450-460, 1999.

D. Kuhl. Near wall investigation of three dimensional turbulent boundary layers. Master's thesis, Virginia Tech, 2001.

V.K. LaMer and D. Sinclair. An improved homogenous aerosol generator. Technical Report 1668, OSRD, Office of Publication Board, U.S. Department of Commerce, Washington, D.C., 1943.

B. Liu, K. Whitby, and H. Yu. A condensation aerosol generator for producing monodispersed aerosols in the size range, $0.36 \mu$ to $1.3 \mu$. Journal Recherches Atmosphériques, 2:397-406, 1966.

K.T. Lowe. The Comprehensive Laser-Doppler Velocimeter for FullyResolved Instantaneous Gradient Measurements in Turbulent Flows. PhD thesis, Virginia Tech, 2005. In progress.

M.K. Mazumder. Letter to R.L. Simpson, 25 February 1981.

M.K. Mazumder, C.W. Blevins, and K.J. Kirsch. Wind tunnel flow seeding for laser velocimetry applications. In Minnesota Symposium on Laser Anemometry Proceedings, pages 328-339, 22-24 October 1975.

J. Meyers. Generation of particles and seeding. In Laser Velocimetry. Lecture Series 1991-08. von Karman Institute for Fluid Dynamics, 10-14 June 1991.

Sonimist. Misonix, Inc., 2002. From webpage: www.misonix.com.

L.F. Moody. Friction factors for pipe flow. Transactions ASME, 66:671-684, 1944.

H.M. Morris. Flow in rough conduits. Transactions ASCE, 120:373-398, 1955.

D.C.F. Muir. The production of monodispersed aerosols by a LaMer-Sinclair generator. The Annals of Occupational Hygenics, 8:233-238, 1965.

C. Nichols. Preparation of polystyrene microspheres for laser velocimetry in wind tunnels. In NASA Technical Memorandum 89163. NASA Langley Research Center, June 1987. 
J. Nikuradse. Strömungsgesetze in rauhen rohren. VDI-Forschungsheft, 361, 1933.

S.M. Olçmen and R.L. Simpson. A five-velocity component laser-doppler velocimeter for measurements of a three-dimensional turbulent boundary layer. Measurement Science and Technology, 6:702-716, 1995.

E.M. O'Loughlin. Resistance to flow over boundaries with small roughness concentrations. PhD thesis, University of Iowa, 1965.

A.E. Perry, K.L. Lim, and S.M. Henbest. An experimental study of the turbulence structure in smooth- and rough-wall boundary layers. Journal of Fluid Mechanics, 177:437-466, 1987.

L. Prandtl and H. Schlichting. Über die ausgebildete turbulenz. Proceedings, Second International Congress of Applied Mechanics (Zurich), pages 2675, 1934.

M.R. Raupach. Anomalies in flux-gradient relationships over forest. Boundary Layer Meteorology, 16:467-486, 1979.

M.R. Raupach, A.S. Thom, and I. Edwards. A wind tunnel study of turbulent flow close to regularly arrayed rough surfaces. Boundary Layer Meteorology, 18:373-397, 1980.

M.R. Raupach, R.A. Antonia, and S. Rajagopalan. Rough-wall turbulent boundary layers. Applied Mechanics Reviews, 44:1-25, 1991.

H. Schlichting. Experimentelle untersuchungen zum rauhigkeitsproblem. Ingenieur-Archiv VII, 1:1-34, 1936.

H.L. Seegmiller. Seeding subsonic, transonic and supersonic flows with 0.5 micron polystyrene spheres. In Wind Tunnel Seeding Systems for Laser Velocimeters, volume NASA Conference Publication 2393, pages 179-187. NASA Langley Research Center, 19-20 March 1985.

R.L. Simpson. A generalized correlation of roughness density effects on the turbulent boundary layer. AIAA Journal, 11:242-244, 1973.

P.R. Spalart. Direct simulation of a turbulent boundary layer up to $R e_{\theta}=$ 1410. Journal of Fluid Mechanics, 187:61-98, 1988. 
G. Tang. Measurements of the Tip-Gap Turbulent Flow Structure in a Low Speed Compressor Cascade. PhD thesis, Virginia Tech, 2004.

I. Tani. Turbulent boundary layer development over rough surfaces. Perspectives in Turbulence Studies, pages 223-249, 1987.

A.S. Thom. Momentum absorption by vegetation. Quarterly Journal of the Royal Meteorological Society, 97:414-428, 1971.

C. Tomkins and R. Adrian. The effect of roughness elements on structure in a turbulent boundary layer. In 253rd Annual Meeting of the Division of Fluid Dynamics, American Physical Society, Washington, DC, November 19-21 2000.

Model 9306A Six-Jet Atomizer. TSI Inc., B edition, February 2003.

D.R. Waigh and R.J. Kind. Improved aerodynamic characterization of regular three-dimensional roughness. AIAA Journal, 36:1117-1119, 1998.

R.A. Wooding, E.F. Bradley, and J.K. Marshall. Drag due to regular arrays of varying geometry. Boundary Layer Meteorology, 5:285-308, 1973.

W.J. Yanta. Measurements of aerosol size distributions with a laser doppler velocimeter (ldv). National Bureau of Standards Special Publication 412, pages 73-88, October 1974. 


\section{Appendix A}

\section{Complete Data Plots}

The following figures are a complete set of the plots of the rough wall data. For each roughness configuration, there is a set of centerline plots and midplane plots. Centerline plots refer to the data taken on the steamwise $x$-axis behind a roughness element, and mid-plane plots refer to data taken on the spanwise axis $(x=$ const) located halfway between two rows of roughness elements. The centerline plots omit $W^{+}$mean velocity and Reynolds stresses and triple products that involve an odd-ordered $w$ fluctuation, since these quantities are zero on the centerline. Other than these exceptions, all mean velocities, Reynolds stresses, triple products, turbulent kinetic energy and structure parameters $a_{1}$ and $1 / S$ are shown for the centerline and mid-plane of the nine roughness configurations measured in this research. For plots of the $5.49 \mathrm{~mm}$ spaced straight Gaussian spikes, refer to Appendix D of Bennington (2004). 


\section{A.1 Gaussian Spikes, 5.49 mm Spacing, Stag- gered}
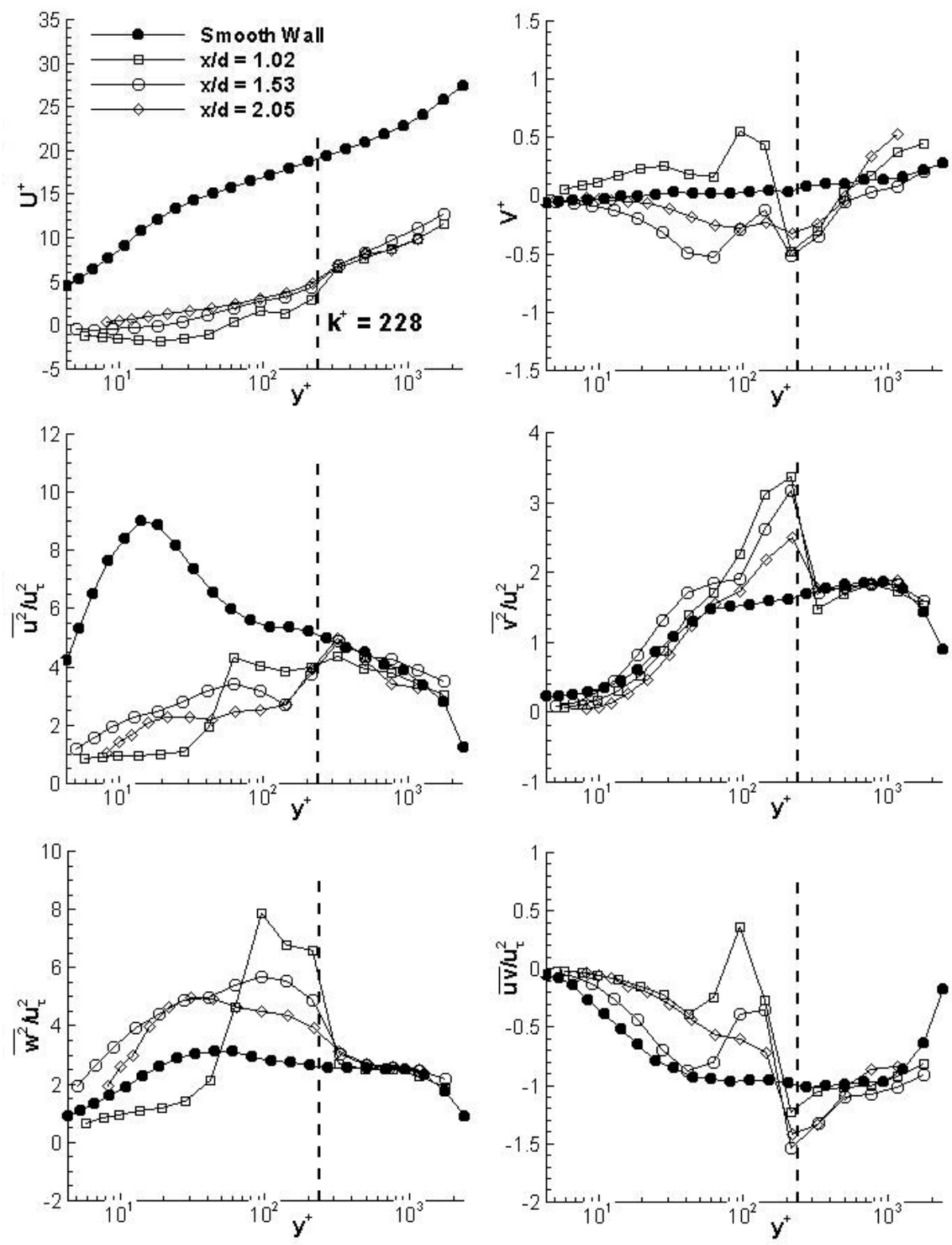

Figure A.1: 5.49 mm spaced staggered Gaussian spikes: Mean velocity and Reynolds stress centerline plots 

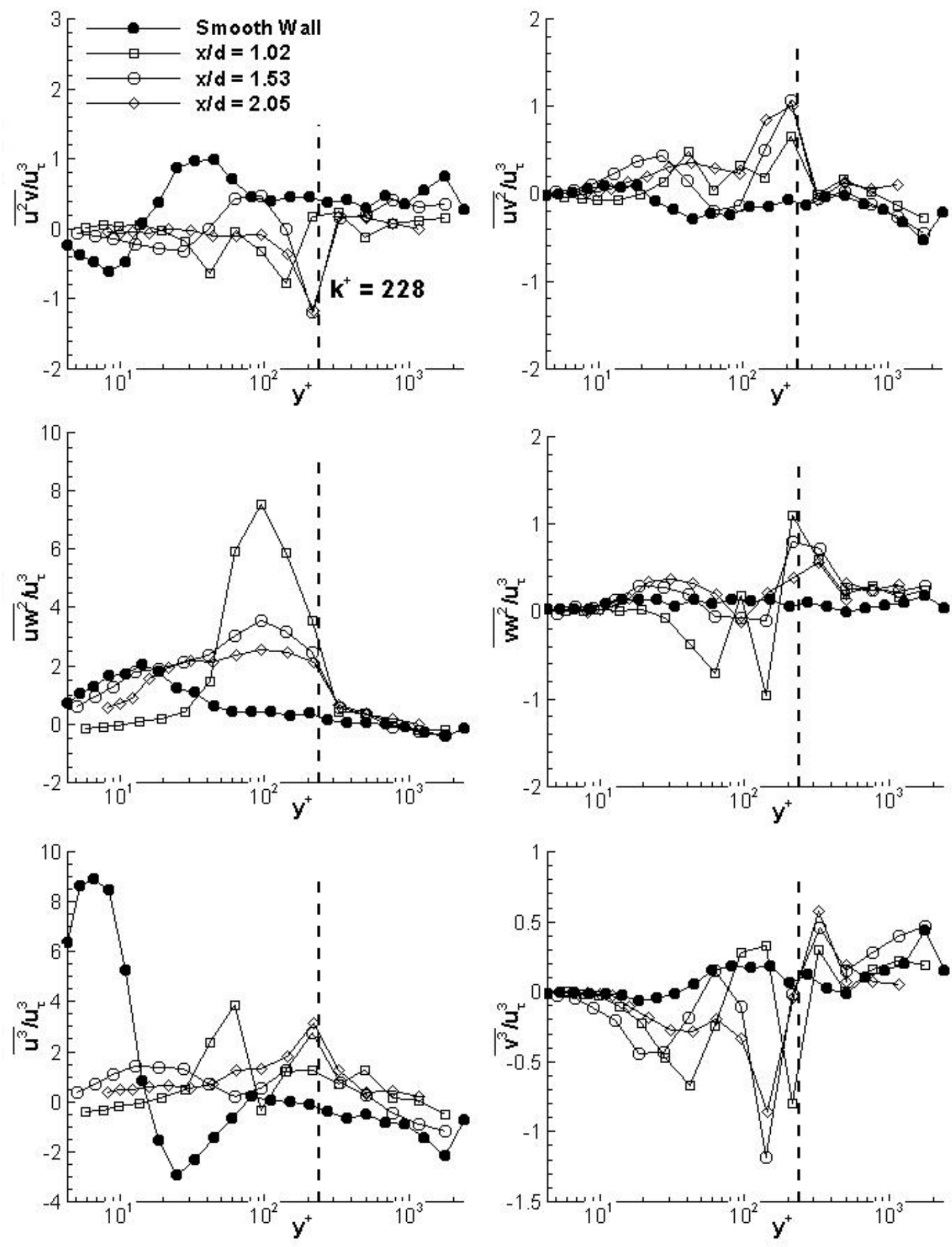

Figure A.2: $5.49 \mathrm{~mm}$ spaced staggered Gaussian spikes: Triple product centerline plots 

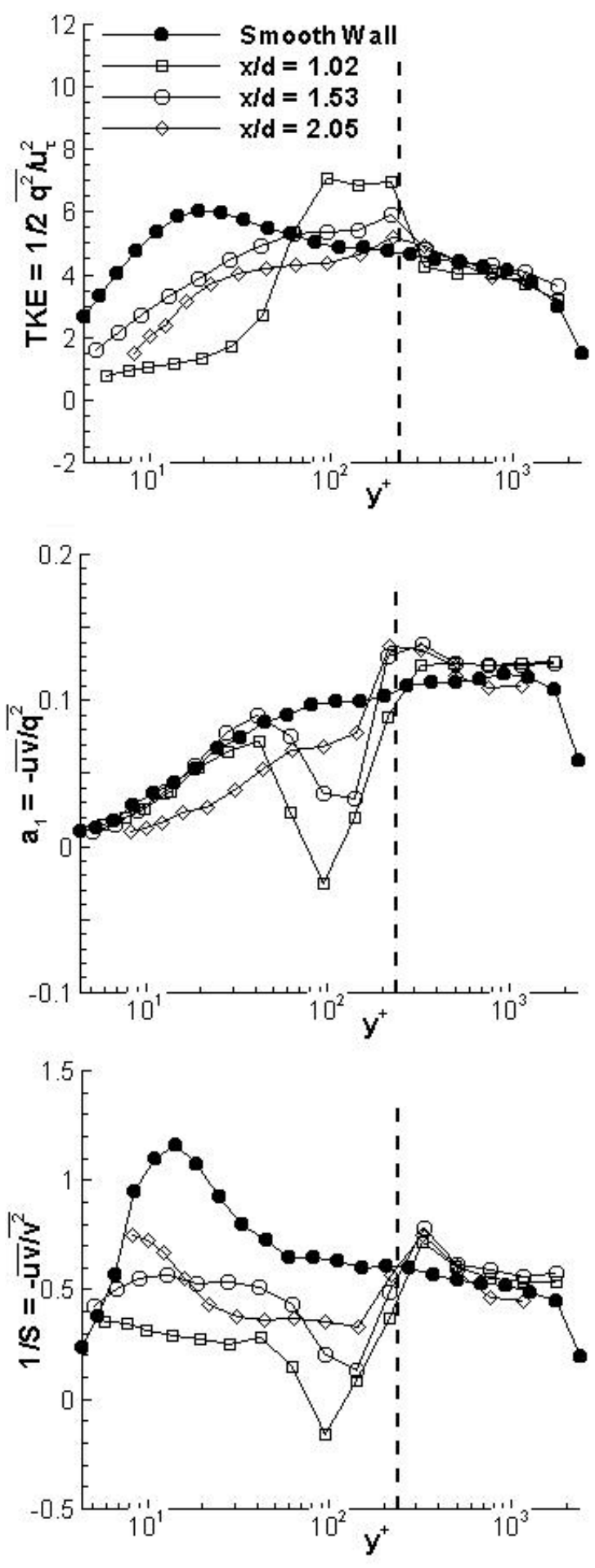

Figure A.3: $5.49 \mathrm{~mm}$ spaced staggered Gaussian spikes: Turbulent kinetic energy and structure parameters $a_{1}$ and $1 / S$ centerline plots 

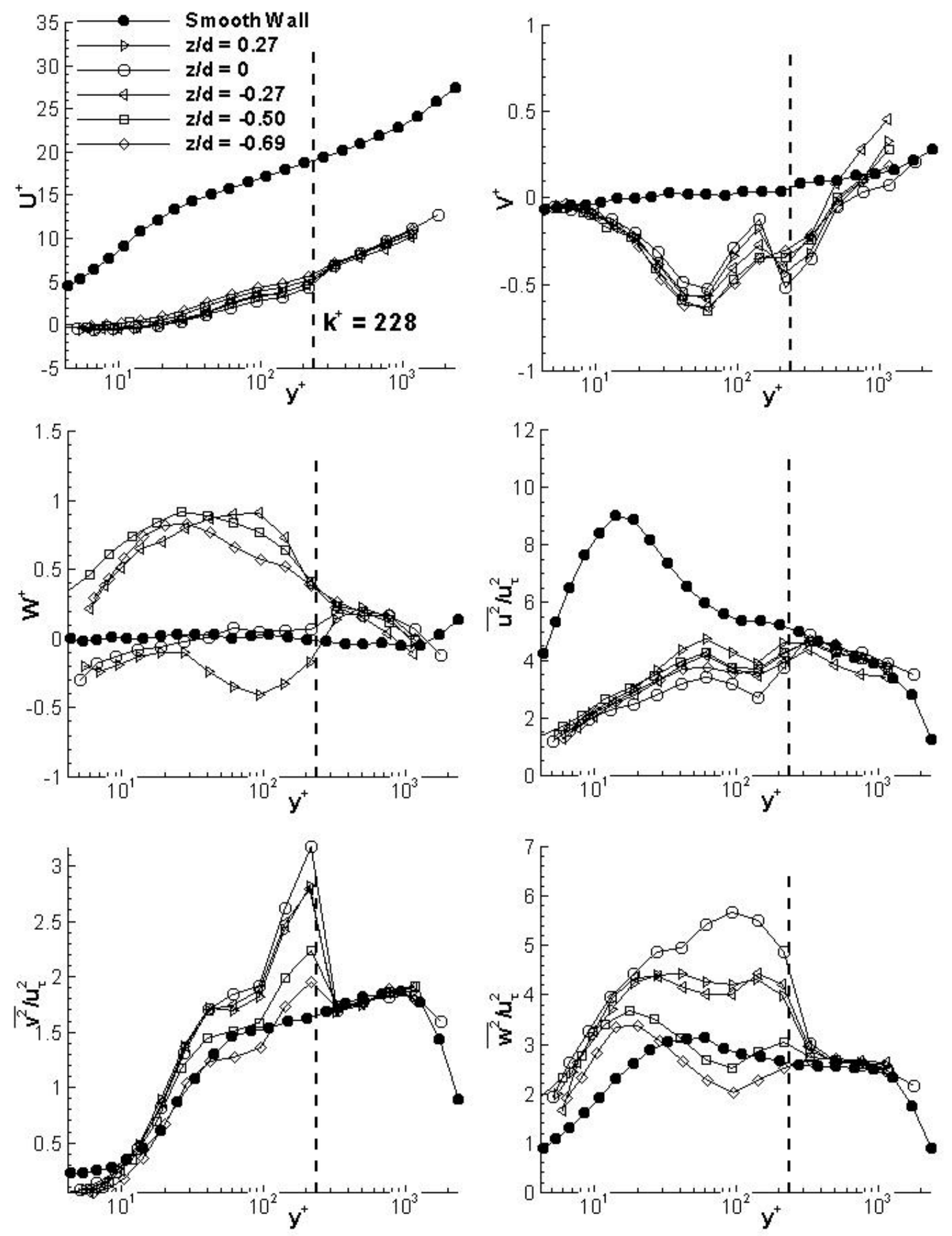

Figure A.4: $5.49 \mathrm{~mm}$ spaced staggered Gaussian spikes: Mean velocity and Reynolds normal stress mid-plane plots 

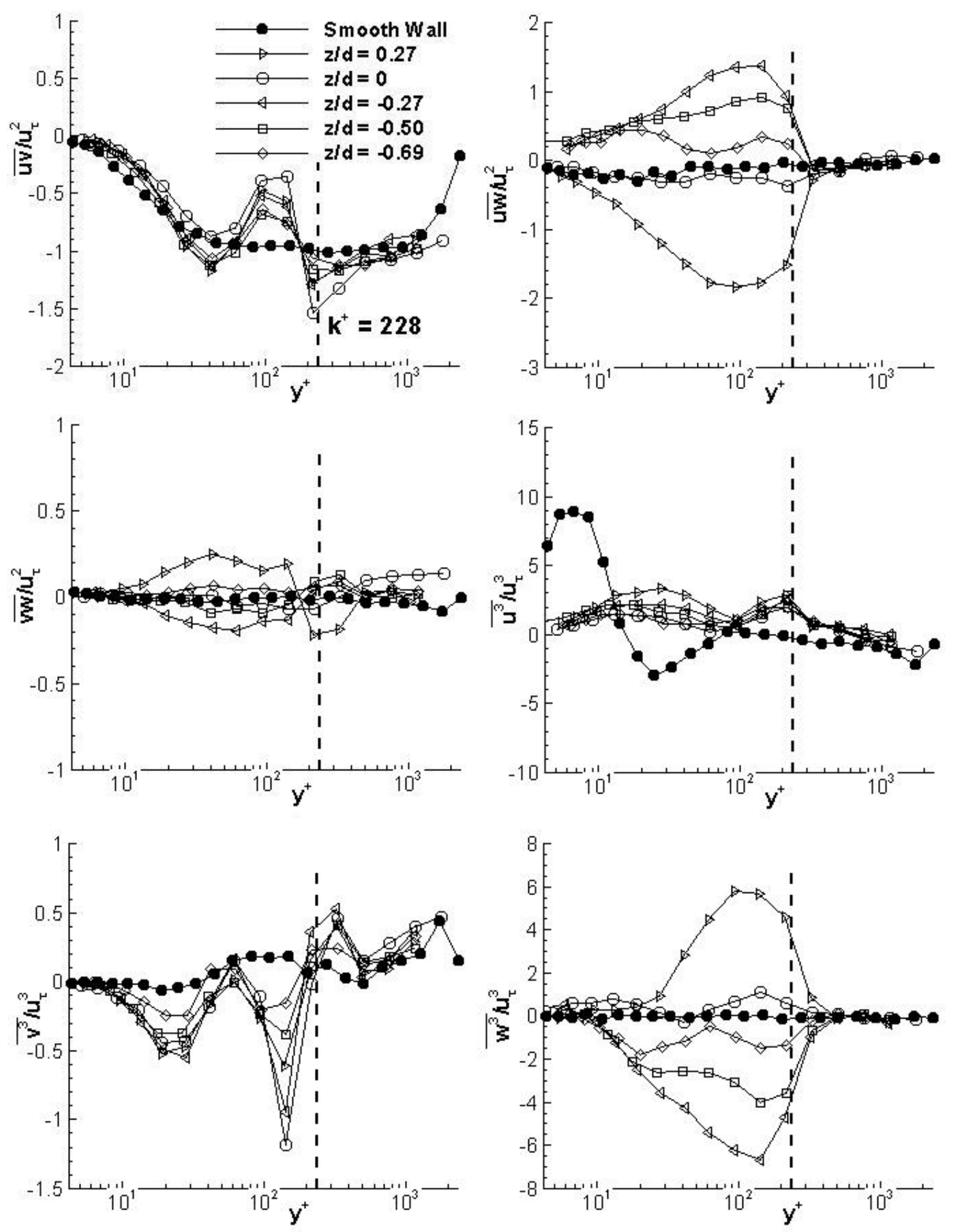

Figure A.5: 5.49 mm spaced staggered Gaussian spikes: Reynolds shear stress and triple product mid-plane plots 

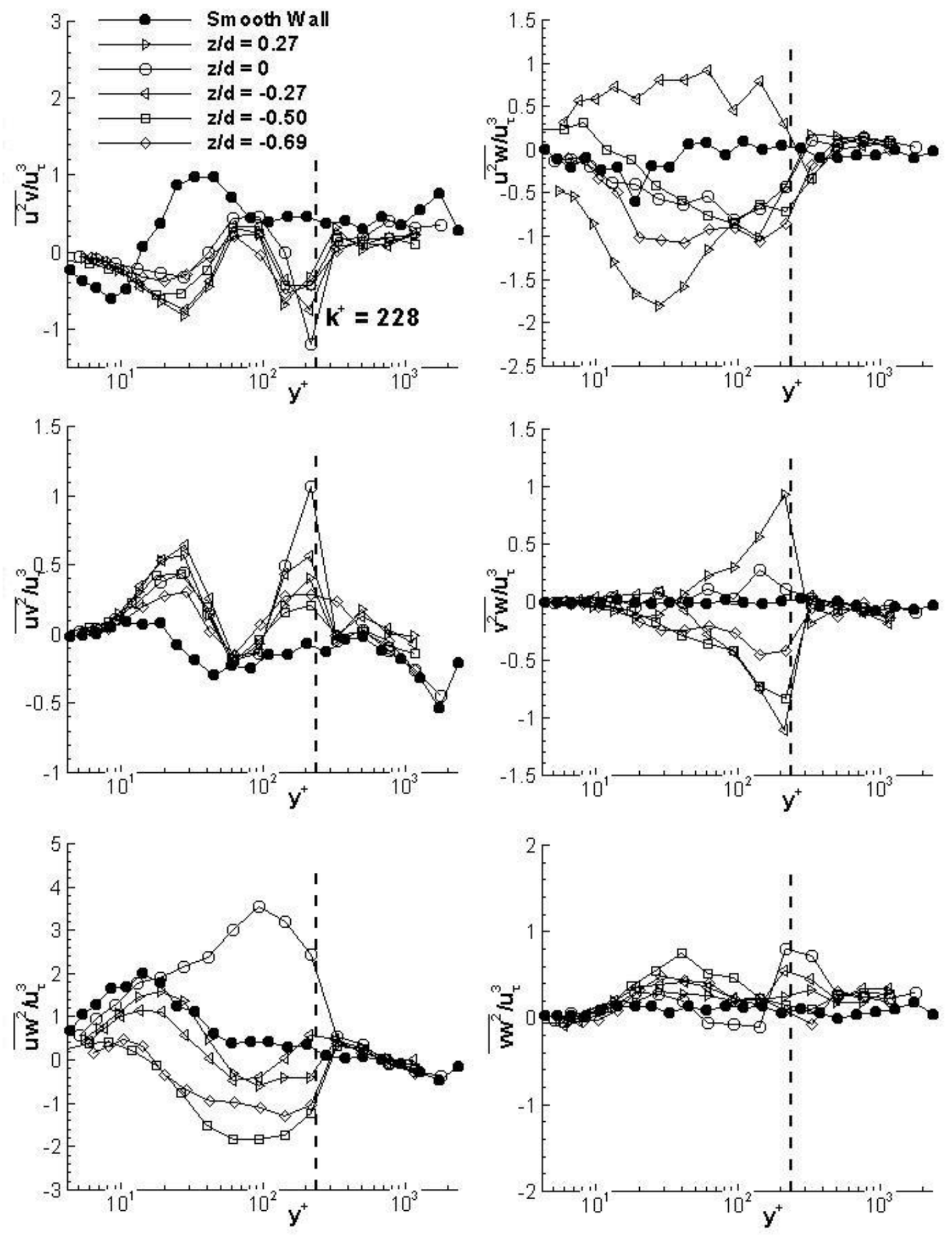

Figure A.6: $5.49 \mathrm{~mm}$ spaced staggered Gaussian spikes: Triple product midplane plots (continued) 

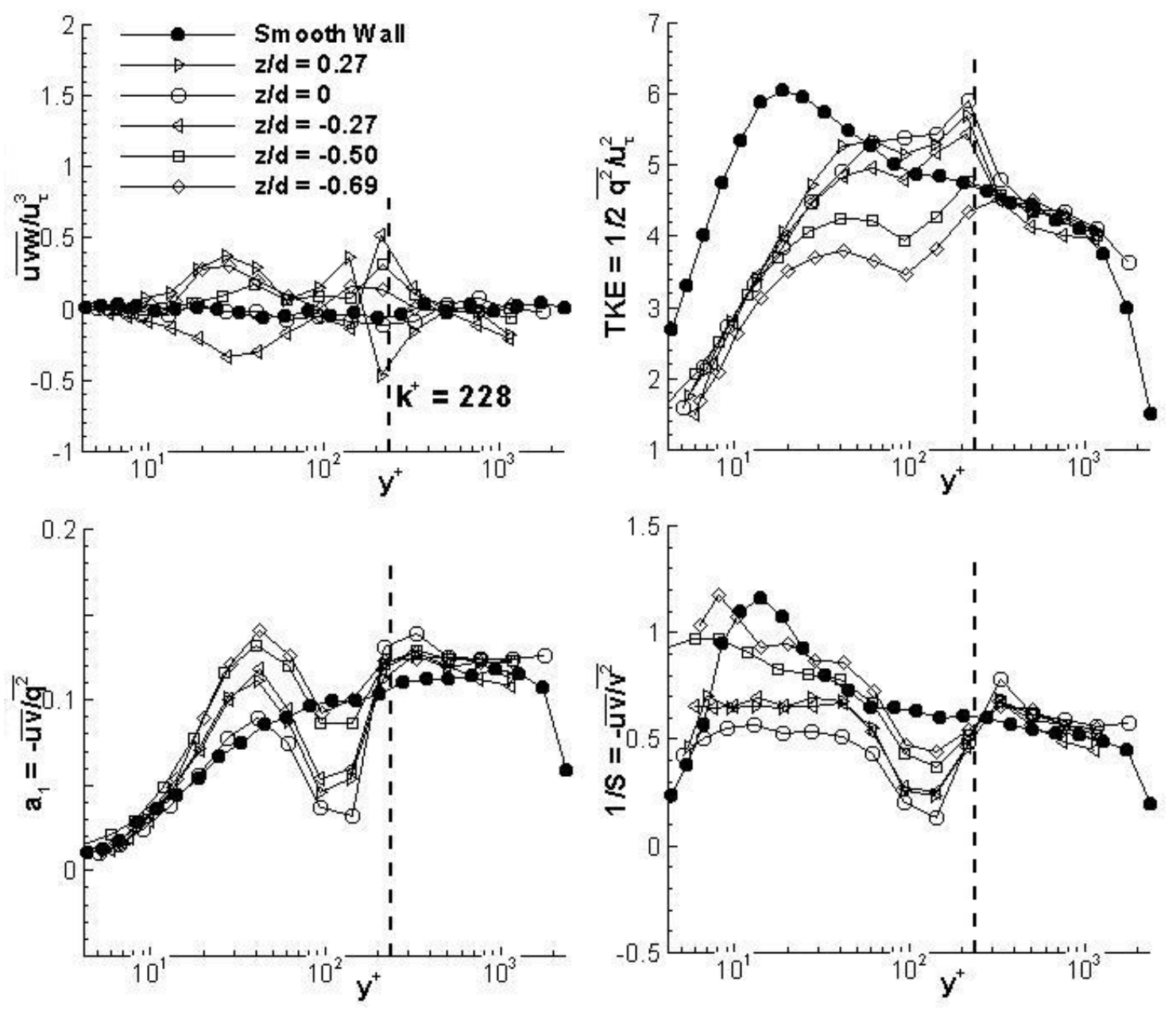

Figure A.7: $5.49 \mathrm{~mm}$ spaced staggered Gaussian spikes: $\overline{u v w} / u_{\tau}^{2}$ triple product, turbulent kinetic energy and structure parameters $a_{1}$ and $1 / S$ mid-plane plots 


\section{A.2 Gaussian Spikes, 8.23 mm Spacing, Straight}
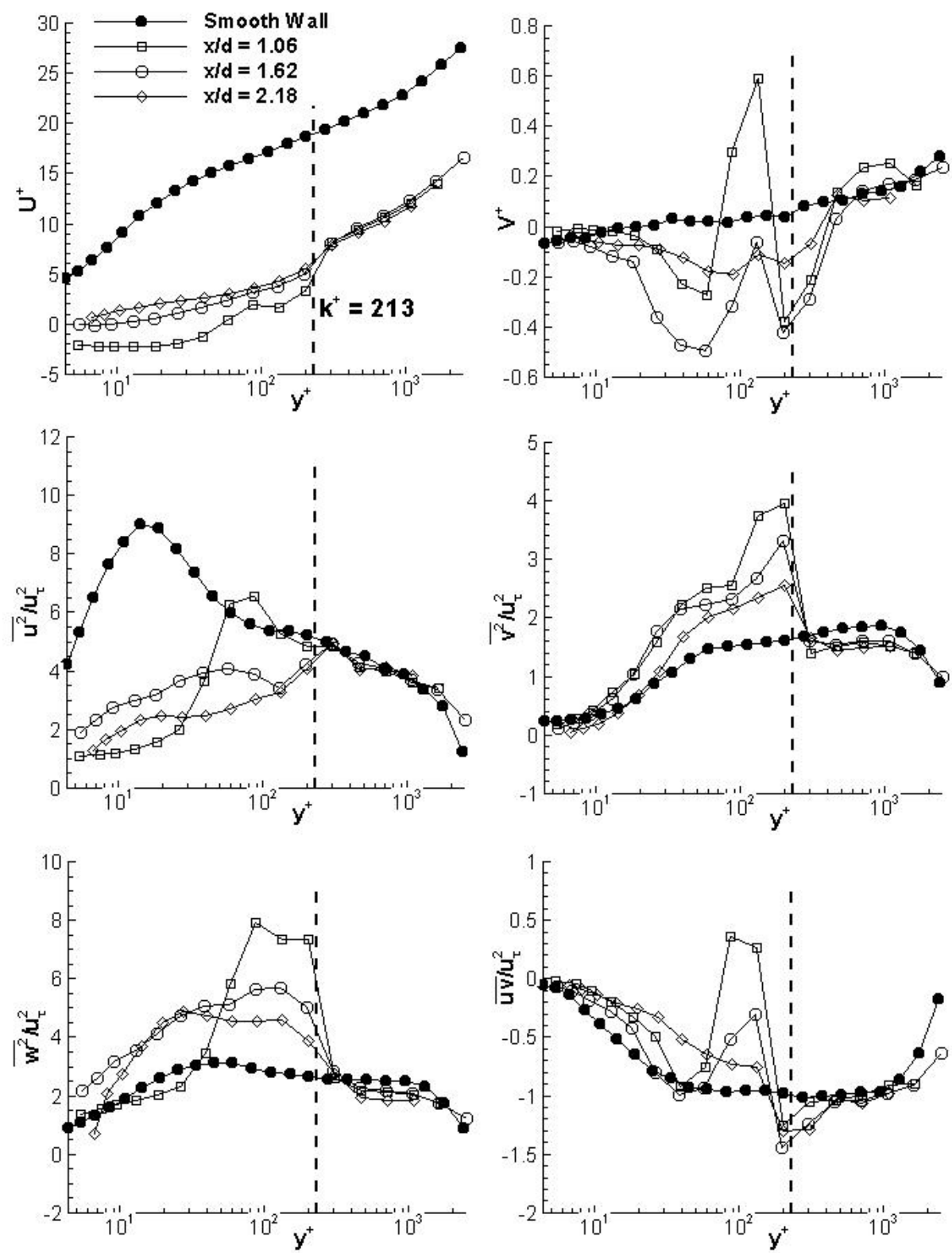

Figure A.8: $8.23 \mathrm{~mm}$ spaced straight Gaussian spikes: Mean velocity and Reynolds stress centerline plots 

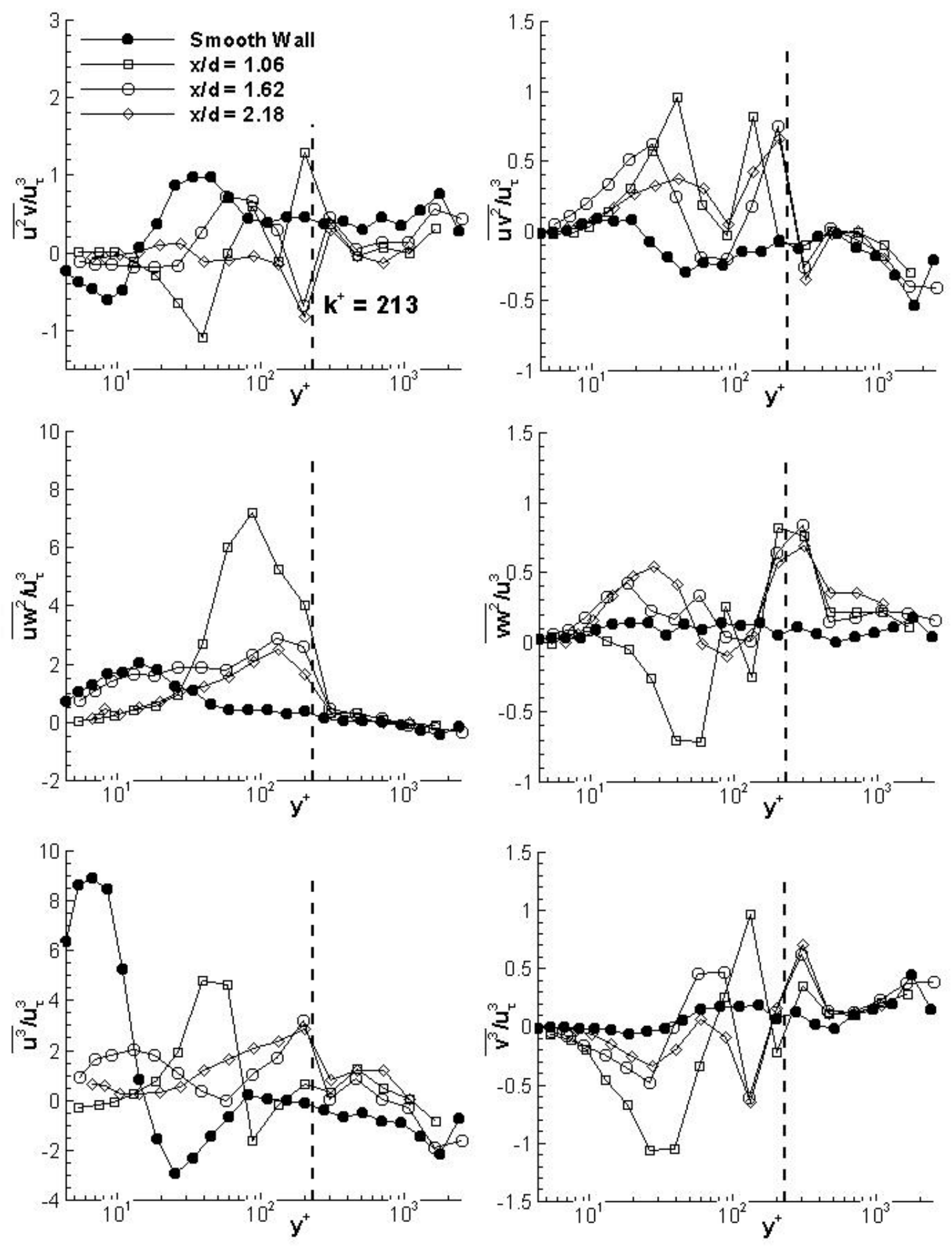

Figure A.9: $8.23 \mathrm{~mm}$ spaced straight Gaussian spikes: Triple product centerline plots 

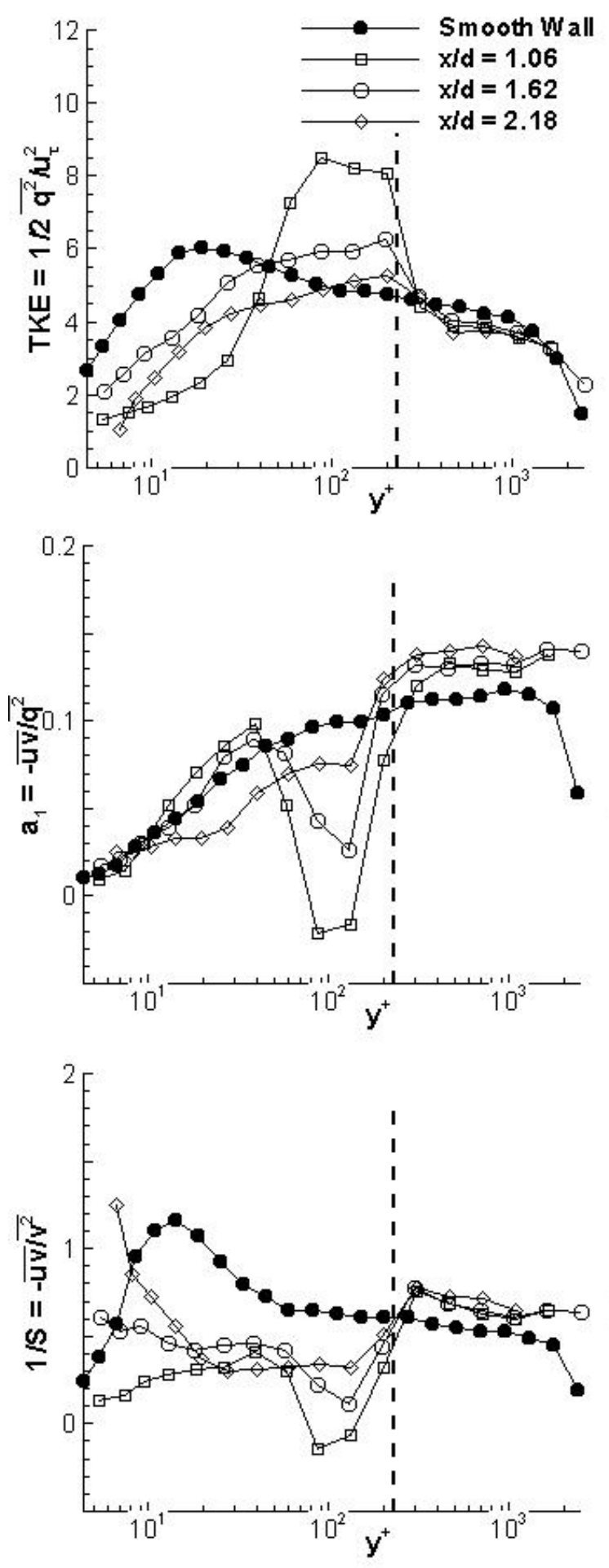

Figure A.10: $8.23 \mathrm{~mm}$ spaced straight Gaussian spikes: Turbulent kinetic energy and structure parameters $a_{1}$ and $1 / S$ centerline plots 

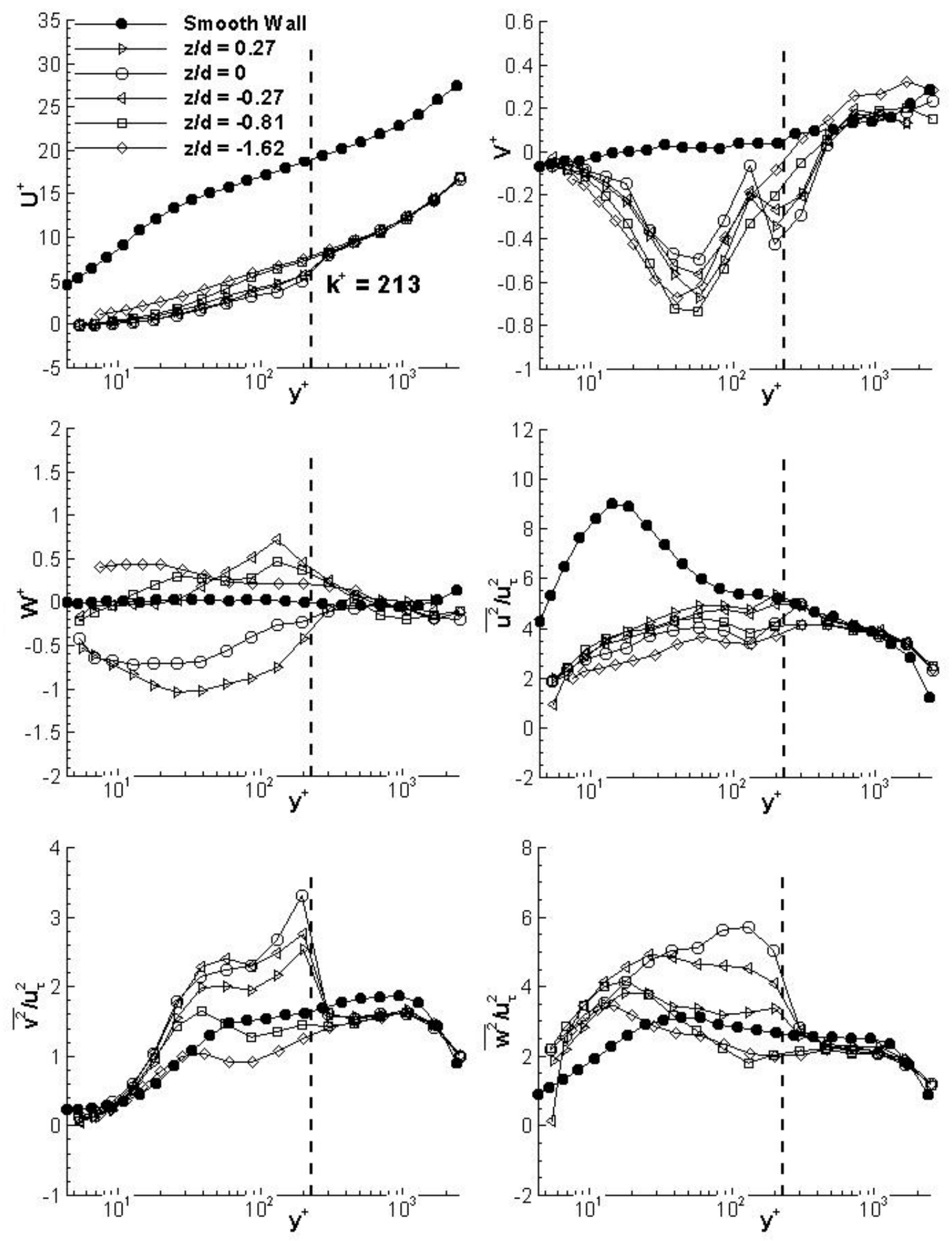

Figure A.11: $8.23 \mathrm{~mm}$ spaced straight Gaussian spikes: Mean velocity and Reynolds normal stress mid-plane plots 

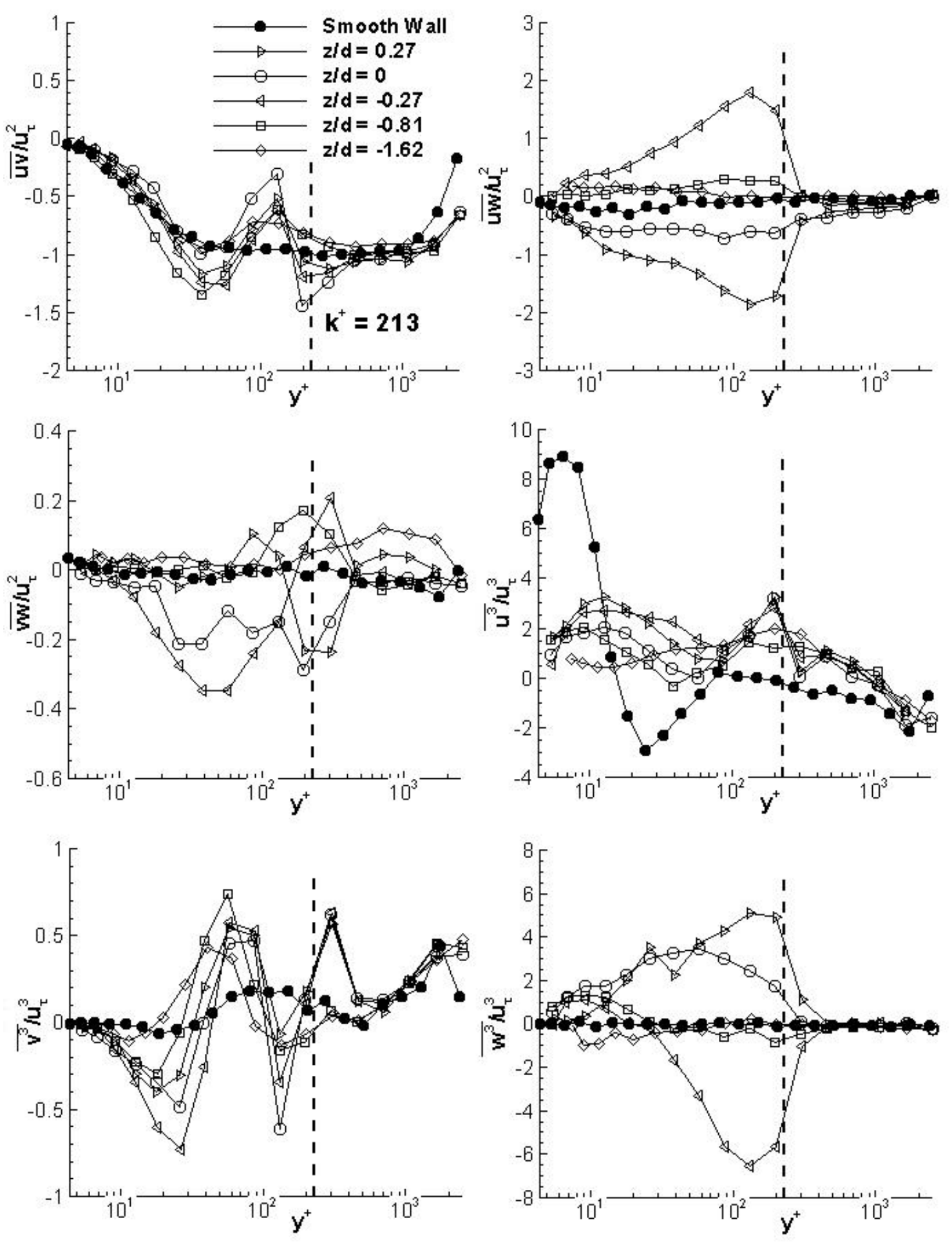

Figure A.12: $8.23 \mathrm{~mm}$ spaced straight Gaussian spikes: Reynolds shear stress and triple product mid-plane plots 

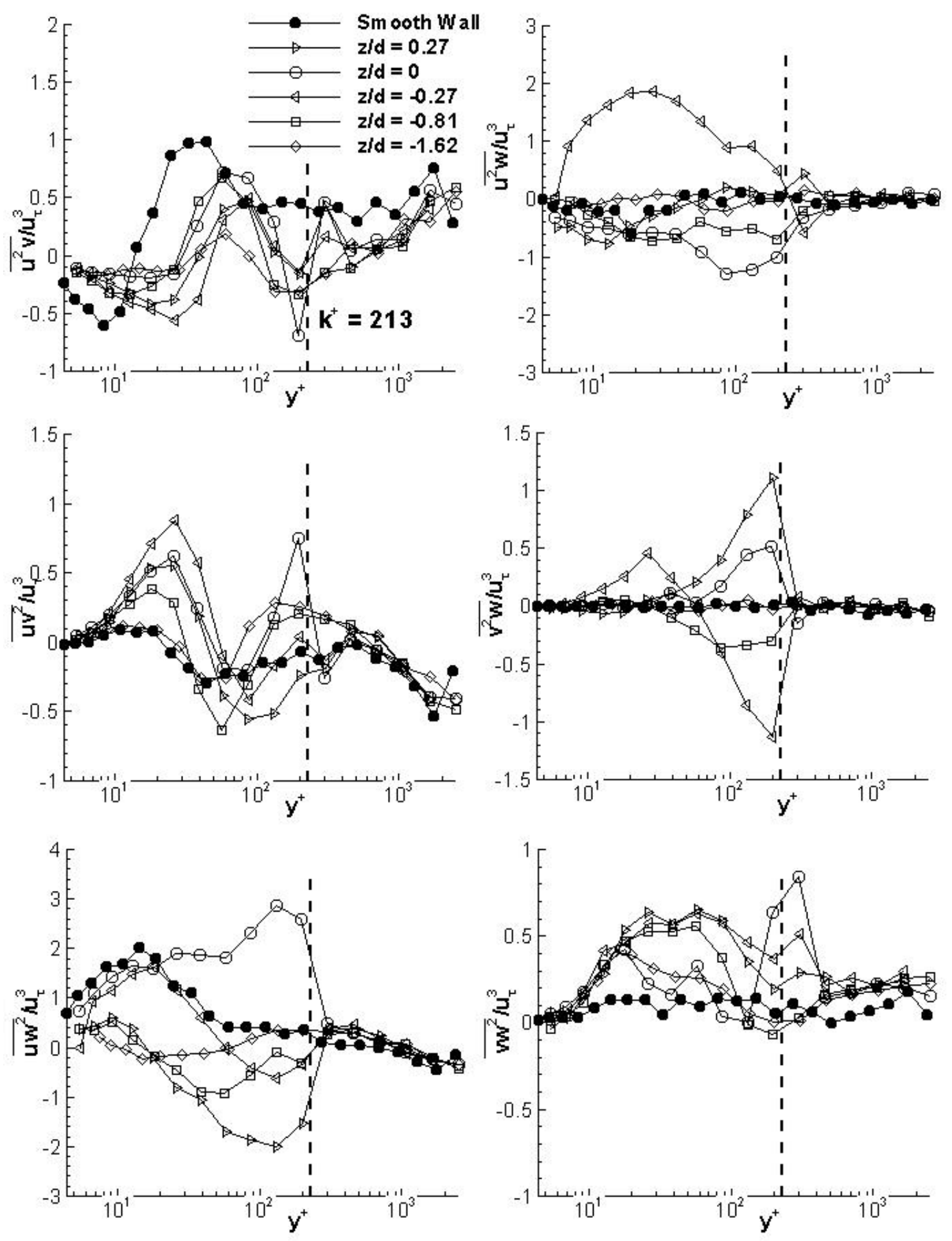

Figure A.13: $8.23 \mathrm{~mm}$ spaced straight Gaussian spikes: Triple product midplane plots (continued) 

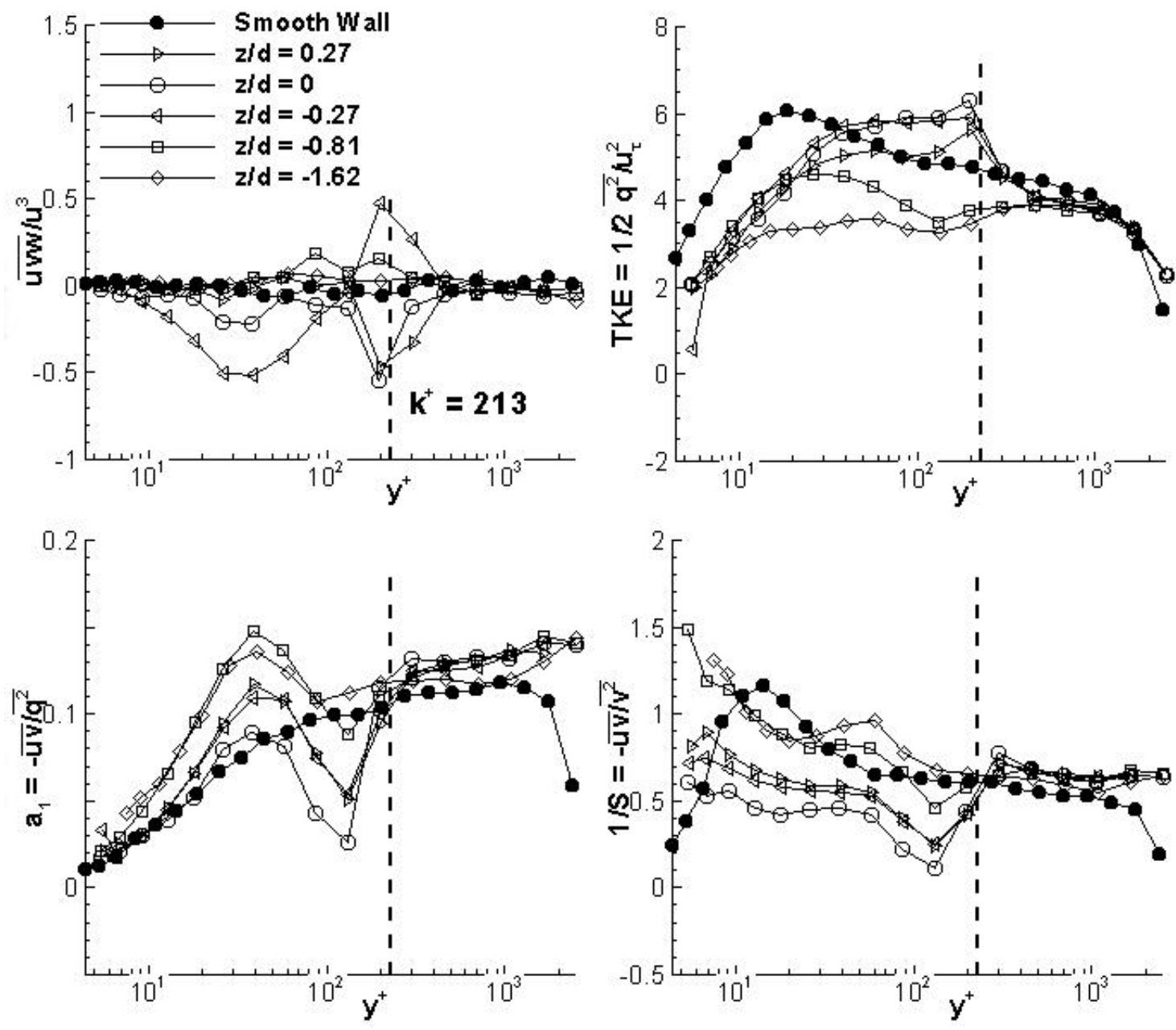

Figure A.14: $8.23 \mathrm{~mm}$ spaced straight Gaussian spikes: $\overline{u v w} / u_{\tau}^{2}$ triple product, turbulent kinetic energy and structure parameters $a_{1}$ and $1 / S$ mid-plane plots 


\section{A.3 Gaussian Spikes, $8.23 \mathrm{~mm}$ Spacing, Stag- gered}
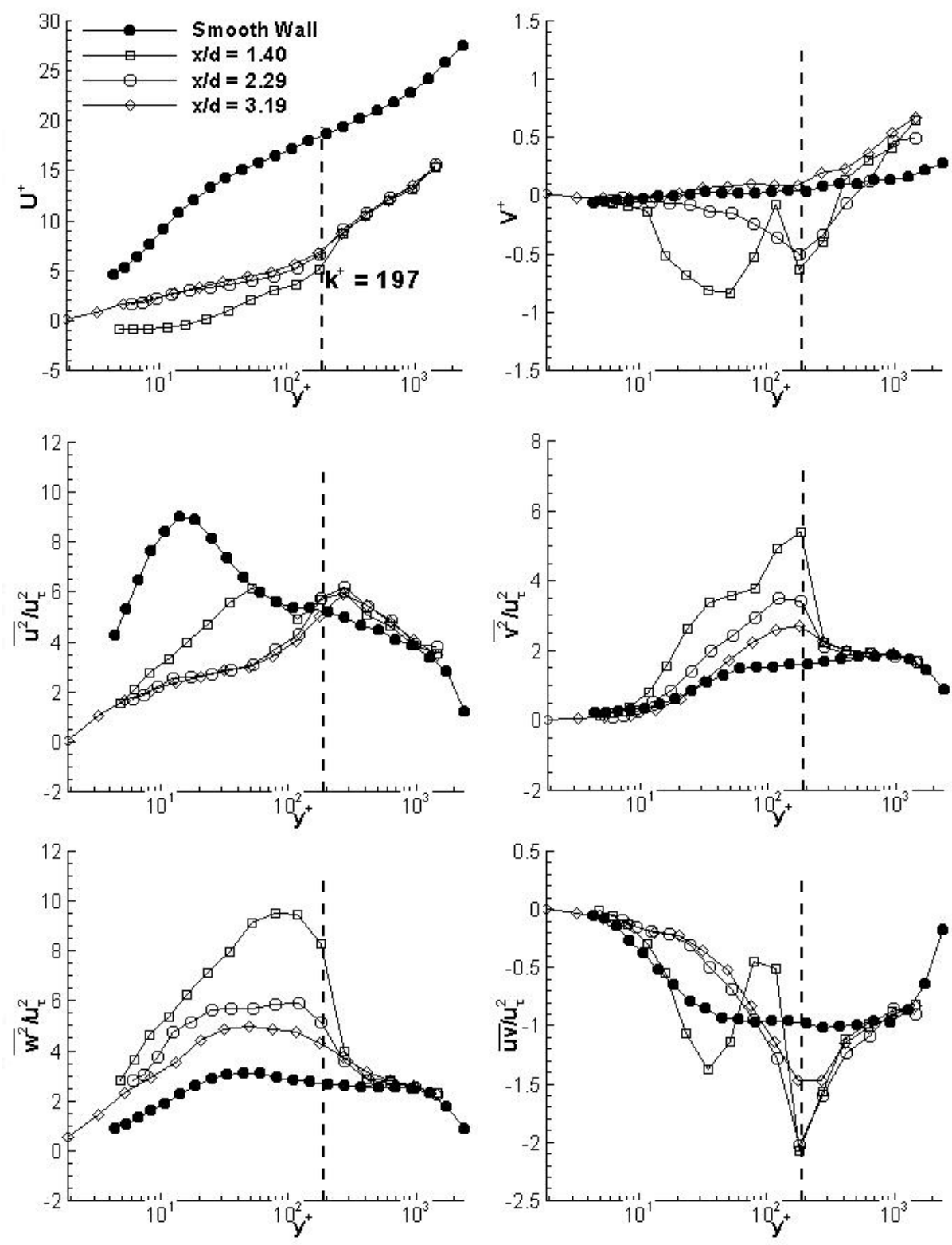

Figure A.15: $8.23 \mathrm{~mm}$ spaced staggered Gaussian spikes: Mean velocity and Reynolds stress centerline plots 

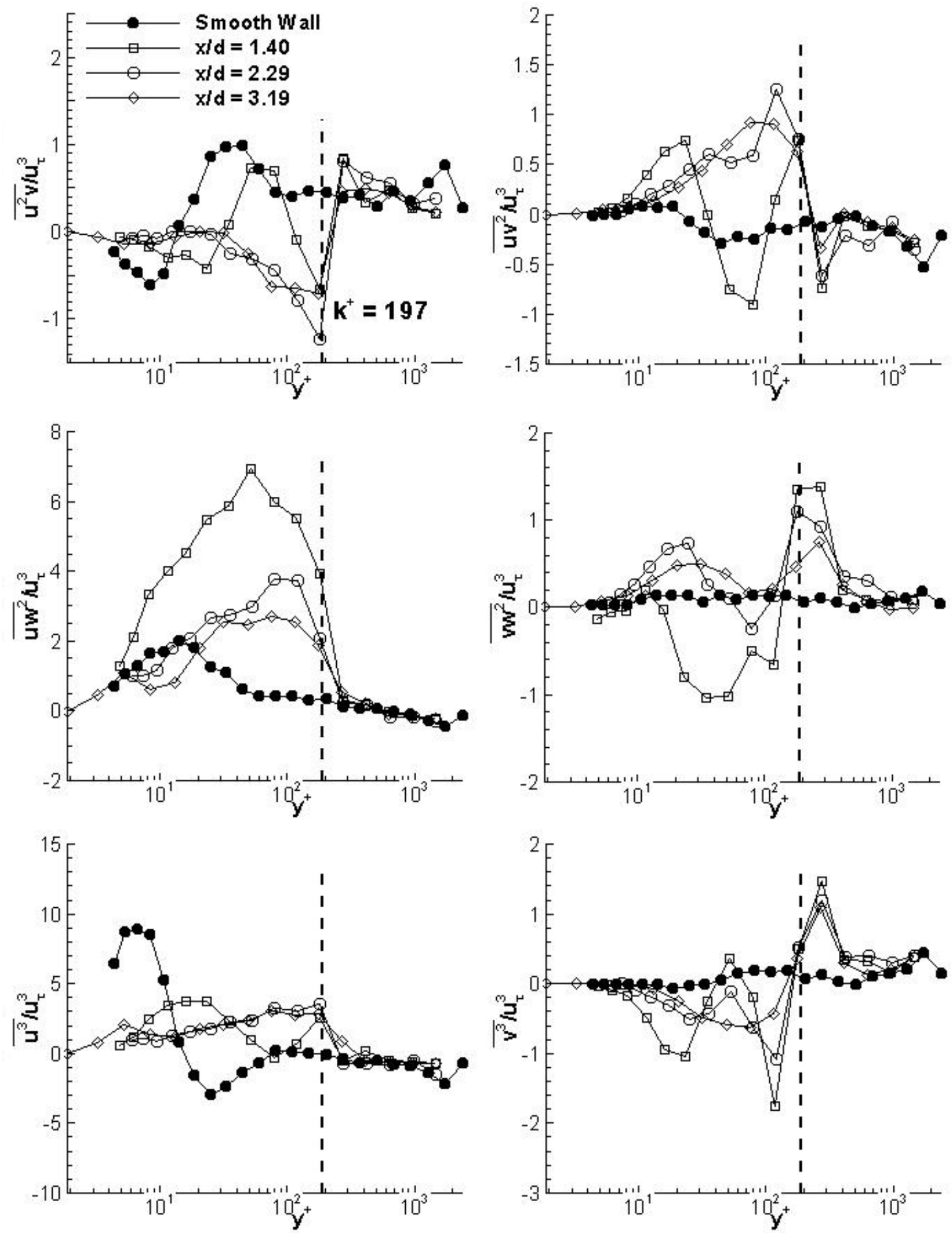

Figure A.16: $8.23 \mathrm{~mm}$ spaced staggered Gaussian spikes: Triple product centerline plots 

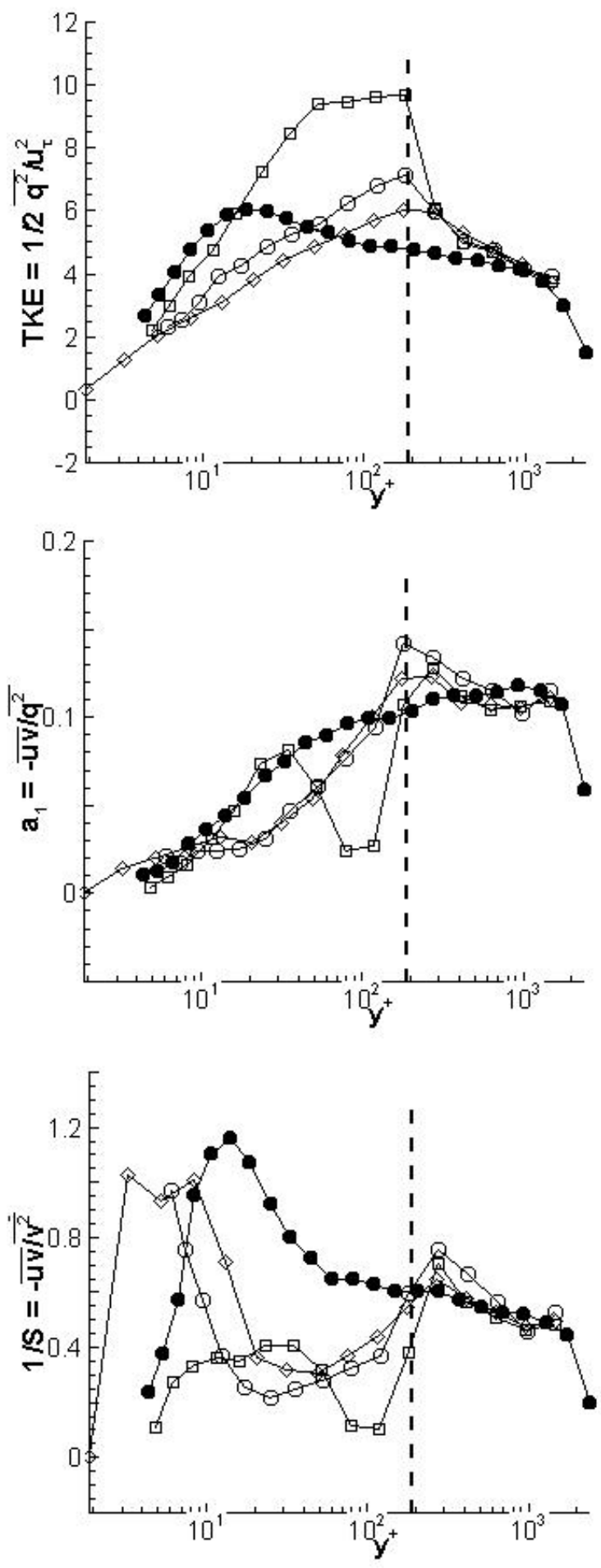

Figure A.17: $8.23 \mathrm{~mm}$ spaced staggered Gaussian spikes: Turbulent kinetic energy and structure parameters $a_{1}$ and $1 / S$ centerline plots 

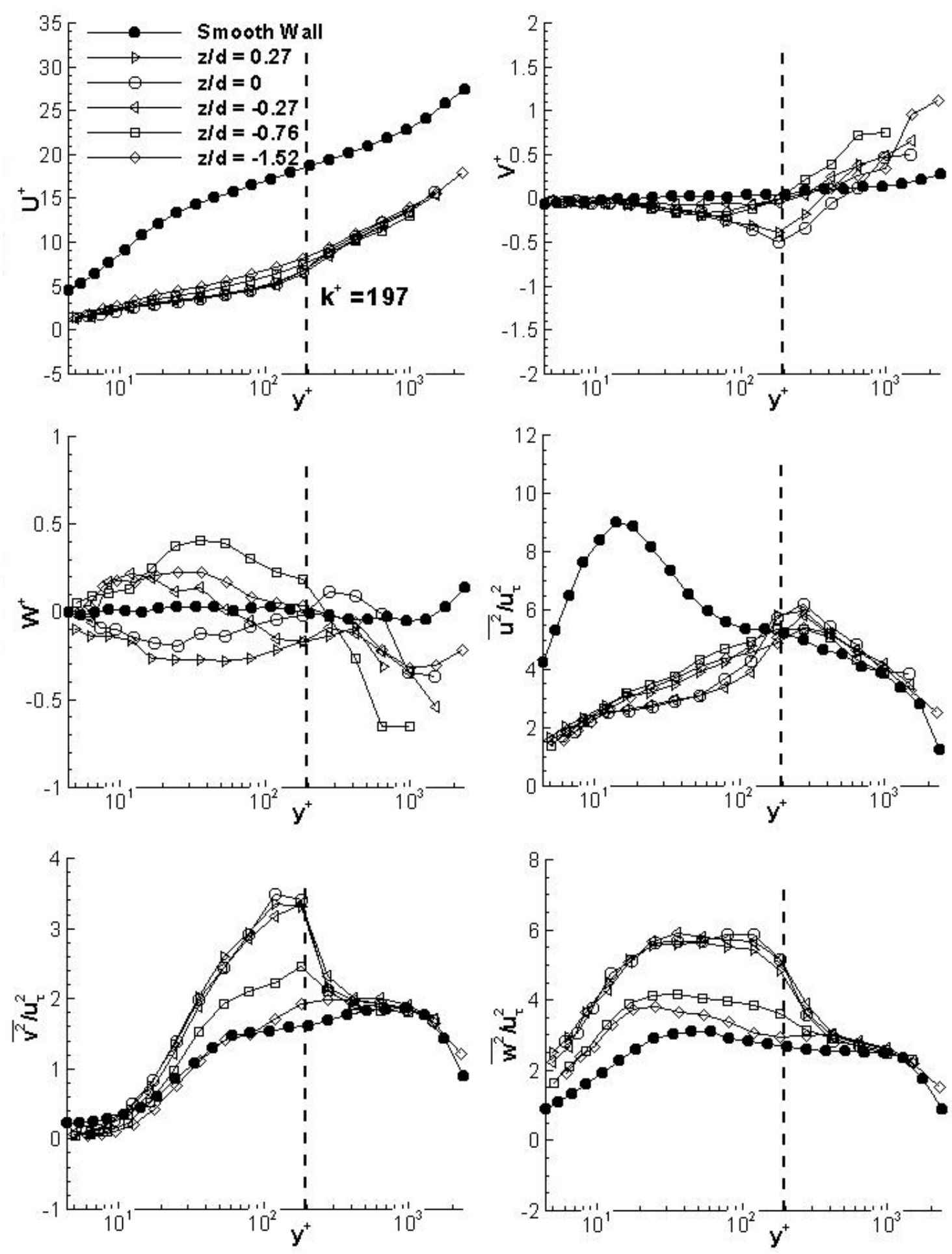

Figure A.18: $8.23 \mathrm{~mm}$ spaced staggered Gaussian spikes: Mean velocity and Reynolds normal stress mid-plane plots 

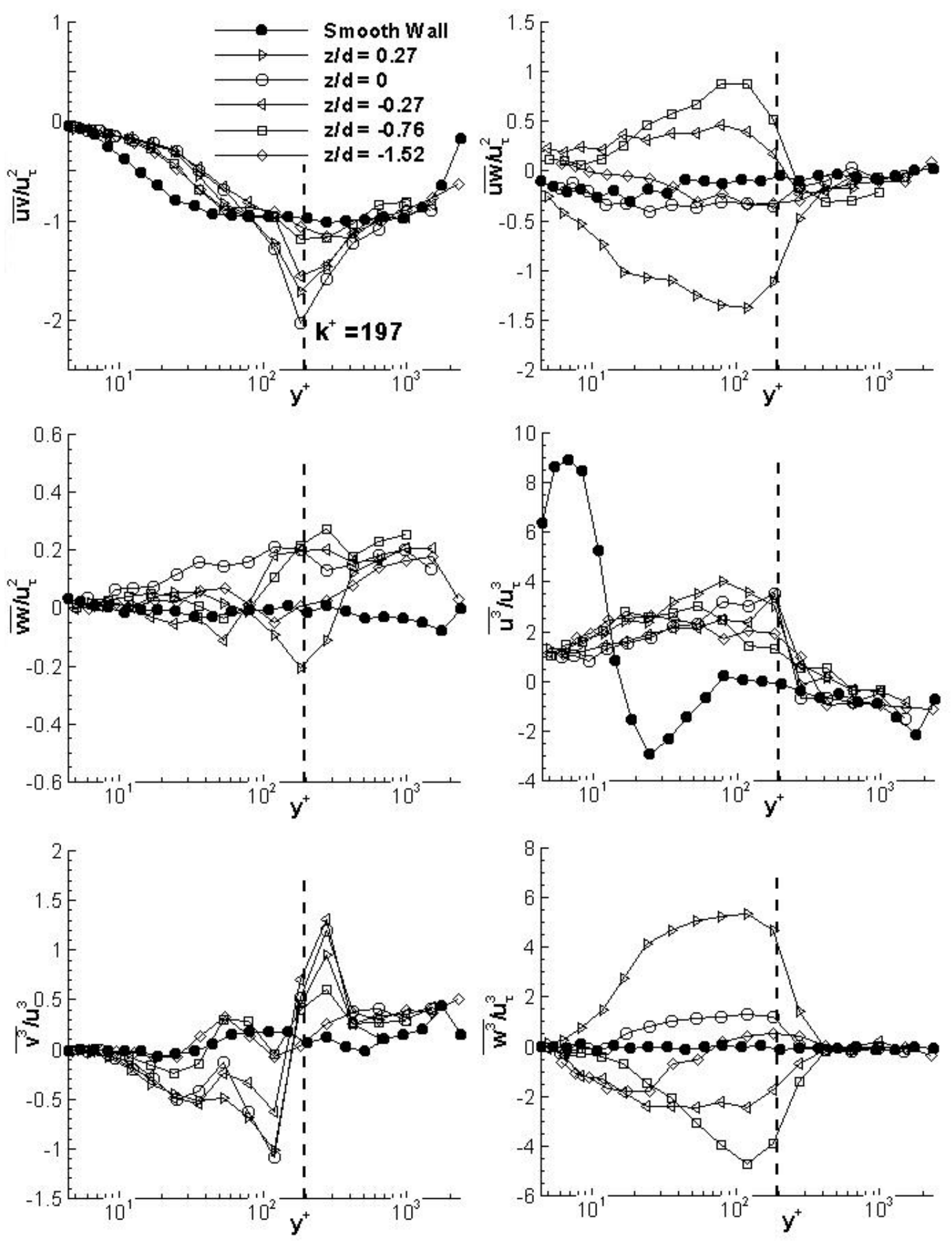

Figure A.19: $8.23 \mathrm{~mm}$ spaced staggered Gaussian spikes: Reynolds shear stress and triple product mid-plane plots 

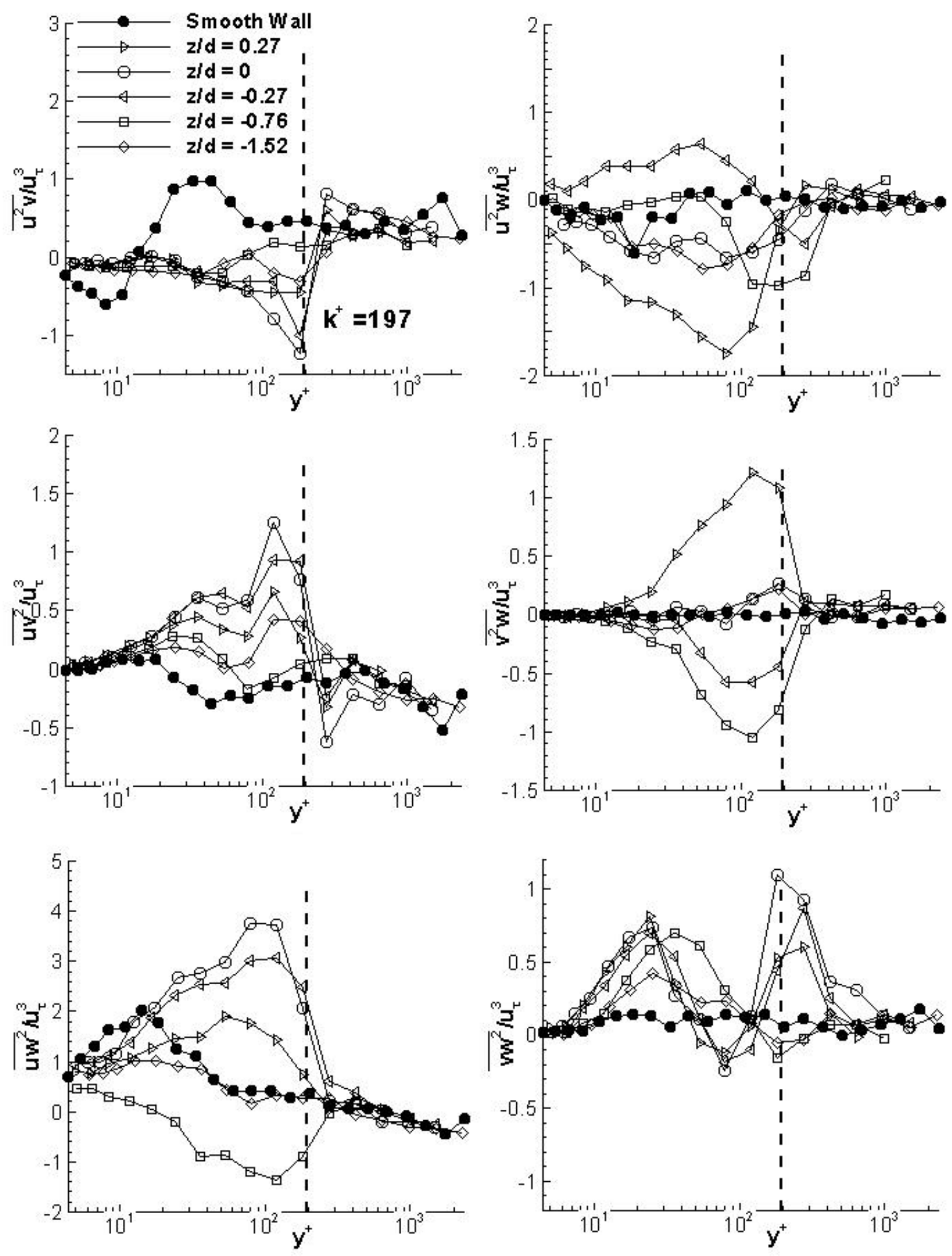

Figure A.20: $8.23 \mathrm{~mm}$ spaced staggered Gaussian spikes: Triple product mid-plane plots (continued) 

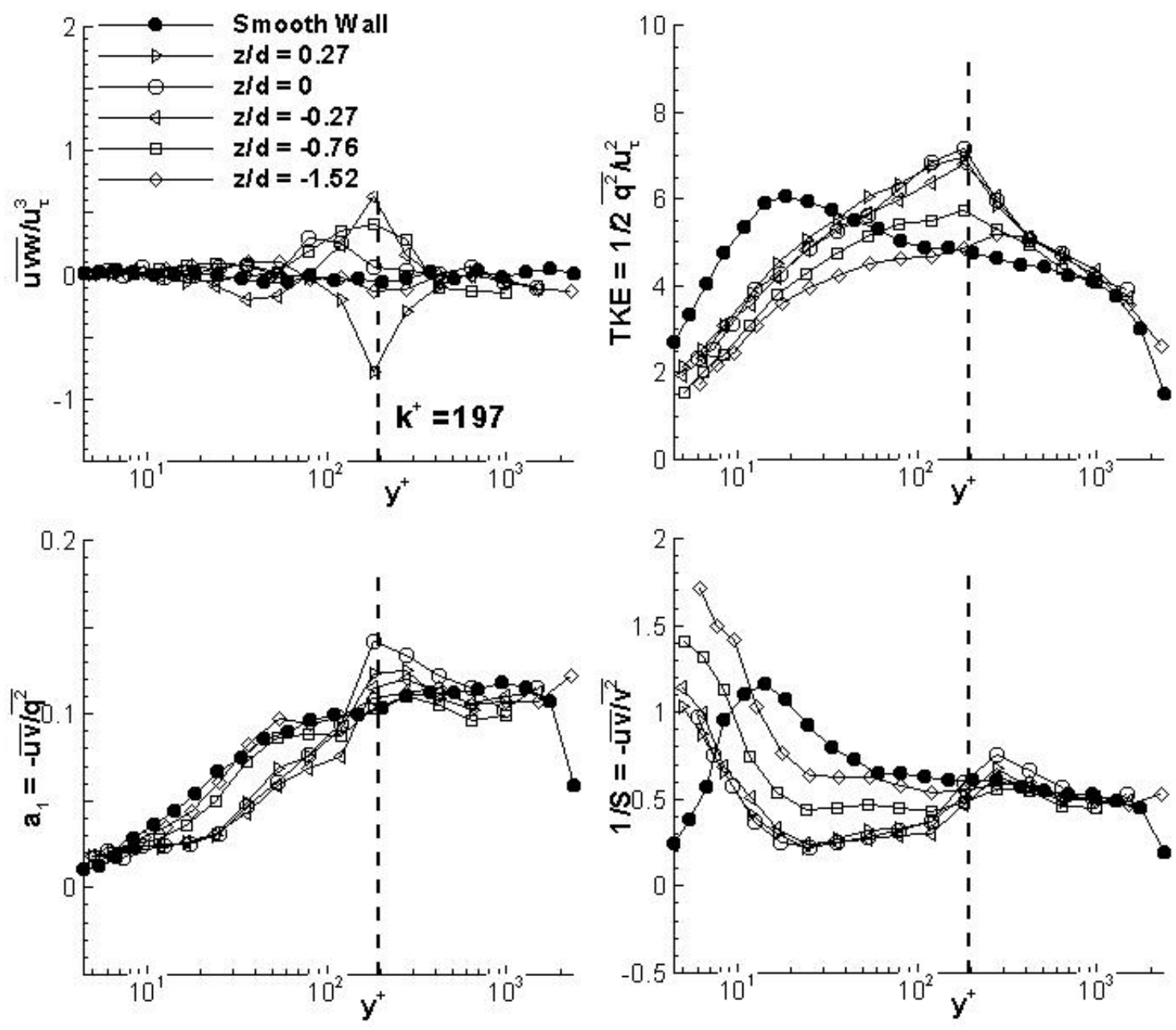

Figure A.21: $8.23 \mathrm{~mm}$ spaced staggered Gaussian spikes: $\overline{u v w} / u_{\tau}^{2}$ triple product, turbulent kinetic energy and structure parameters $a_{1}$ and $1 / S$ mid-plane plots 


\section{A.4 $0.38 \mathrm{~mm}$ Cylinders, Straight}
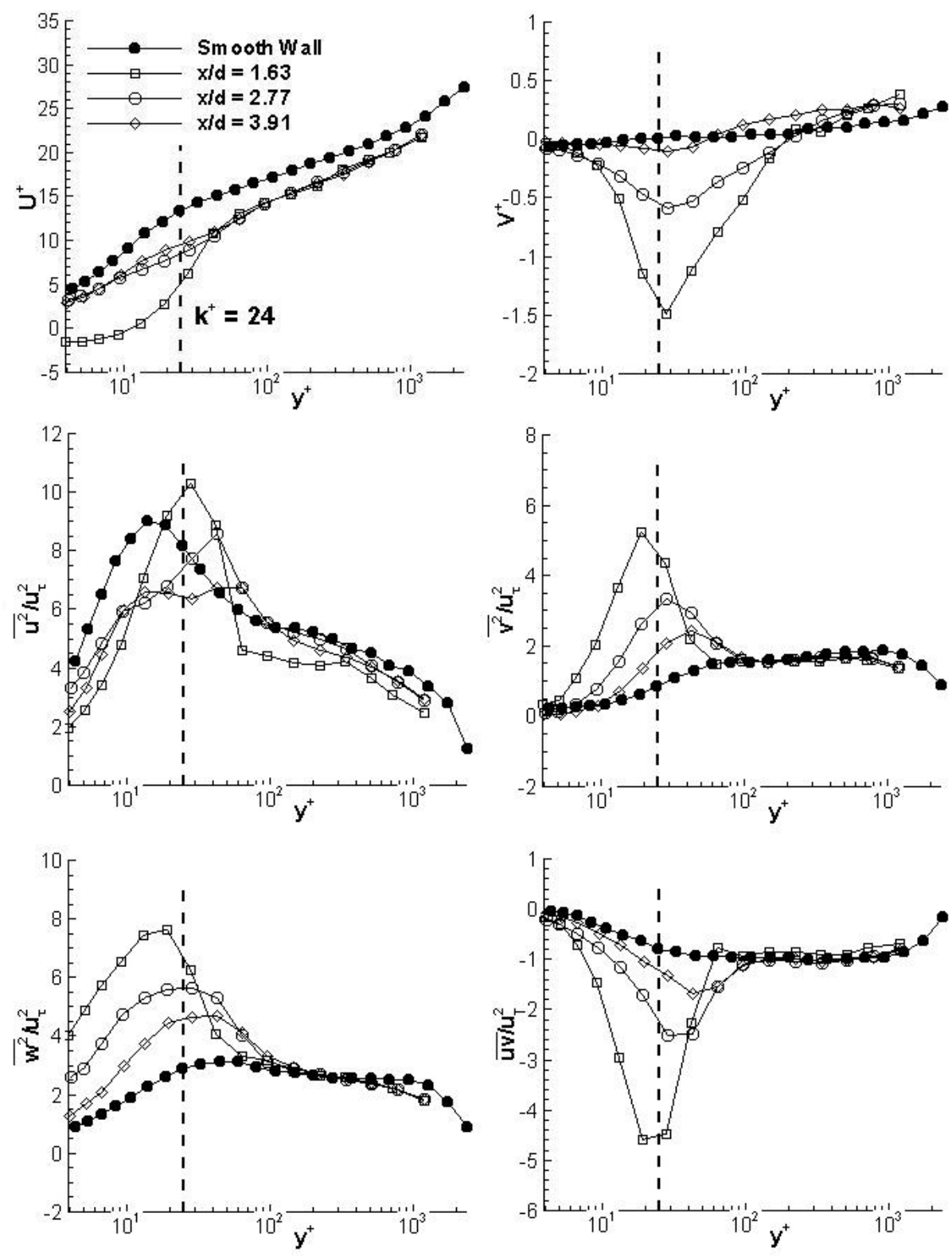

Figure A.22: 0.38 mm Cylinders, straight: Mean velocity and Reynolds stress centerline plots 

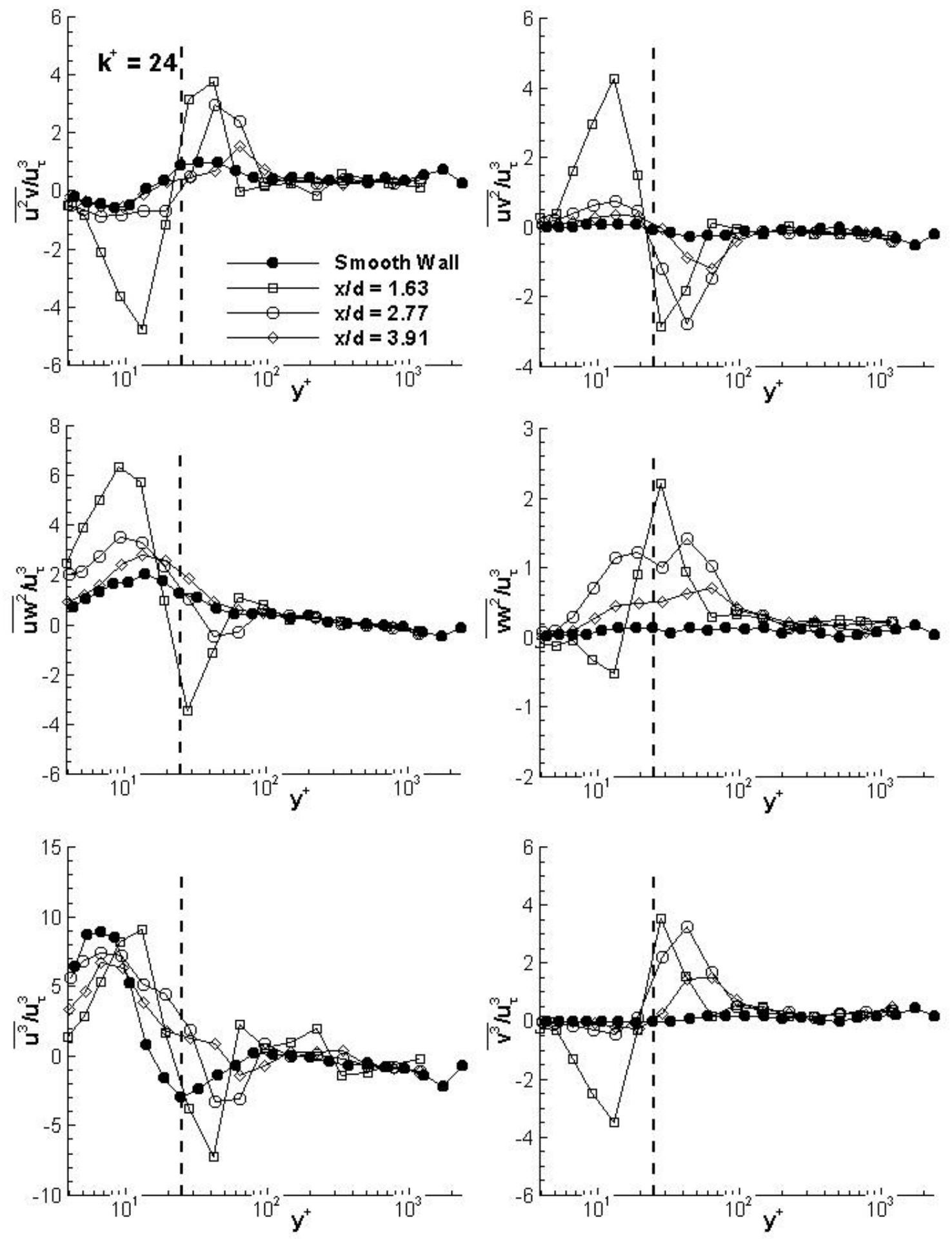

Figure A.23: $0.38 \mathrm{~mm}$ Cylinders, straight: Triple product centerline plots 

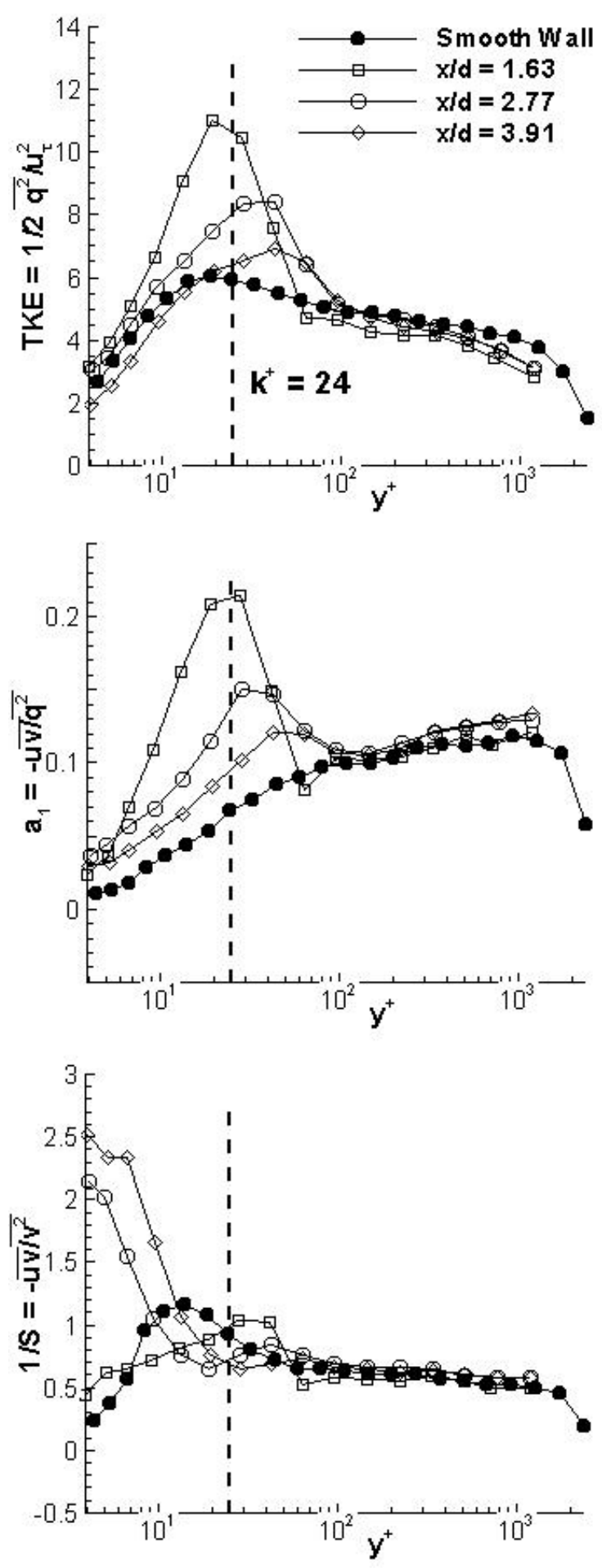

Figure A.24: $0.38 \mathrm{~mm}$ Cylinders, straight: Turbulent kinetic energy and structure parameters $a_{1}$ and $1 / S$ centerline plots 

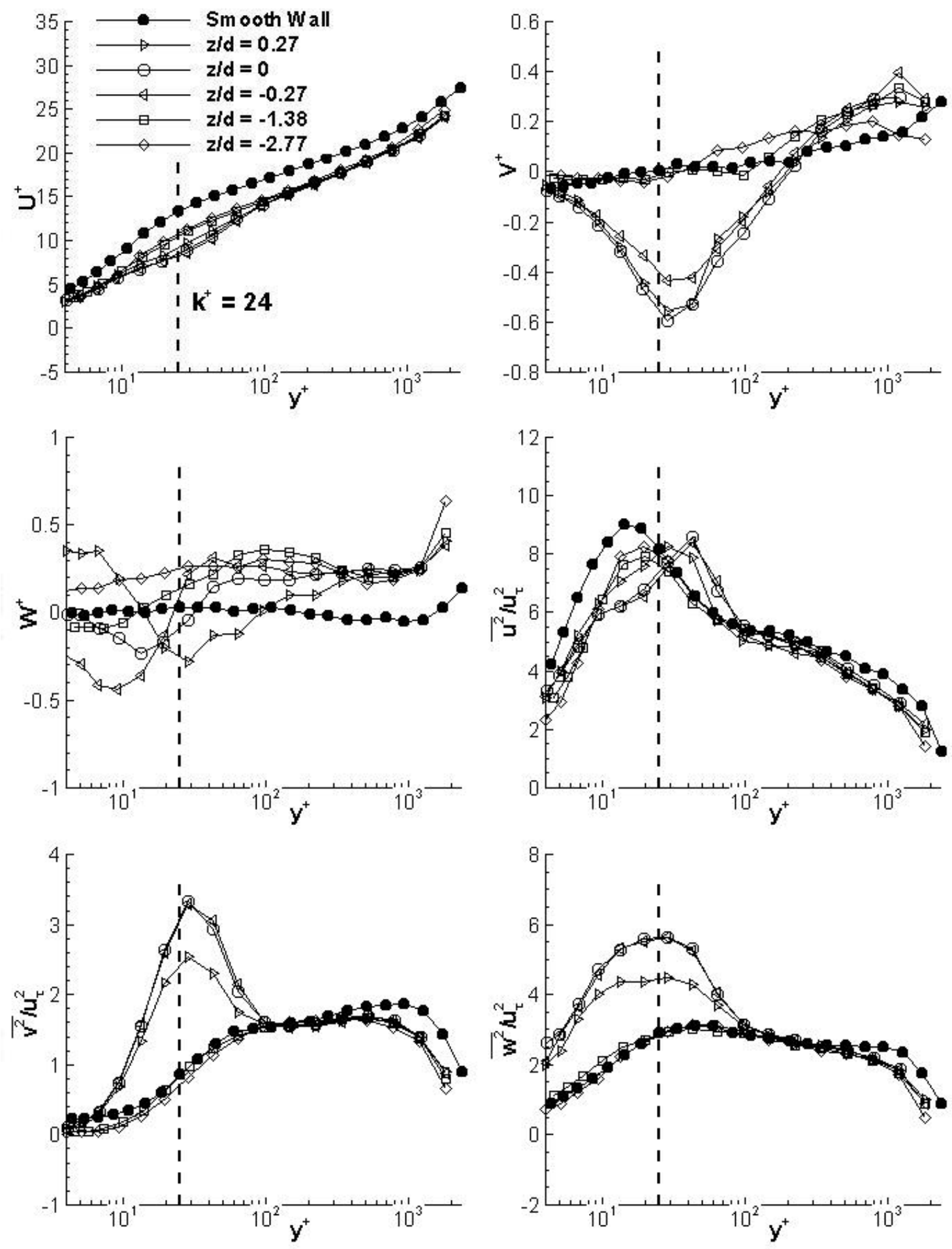

Figure A.25: $0.38 \mathrm{~mm}$ Cylinders, straight: Mean velocity and Reynolds normal stress mid-plane plots 

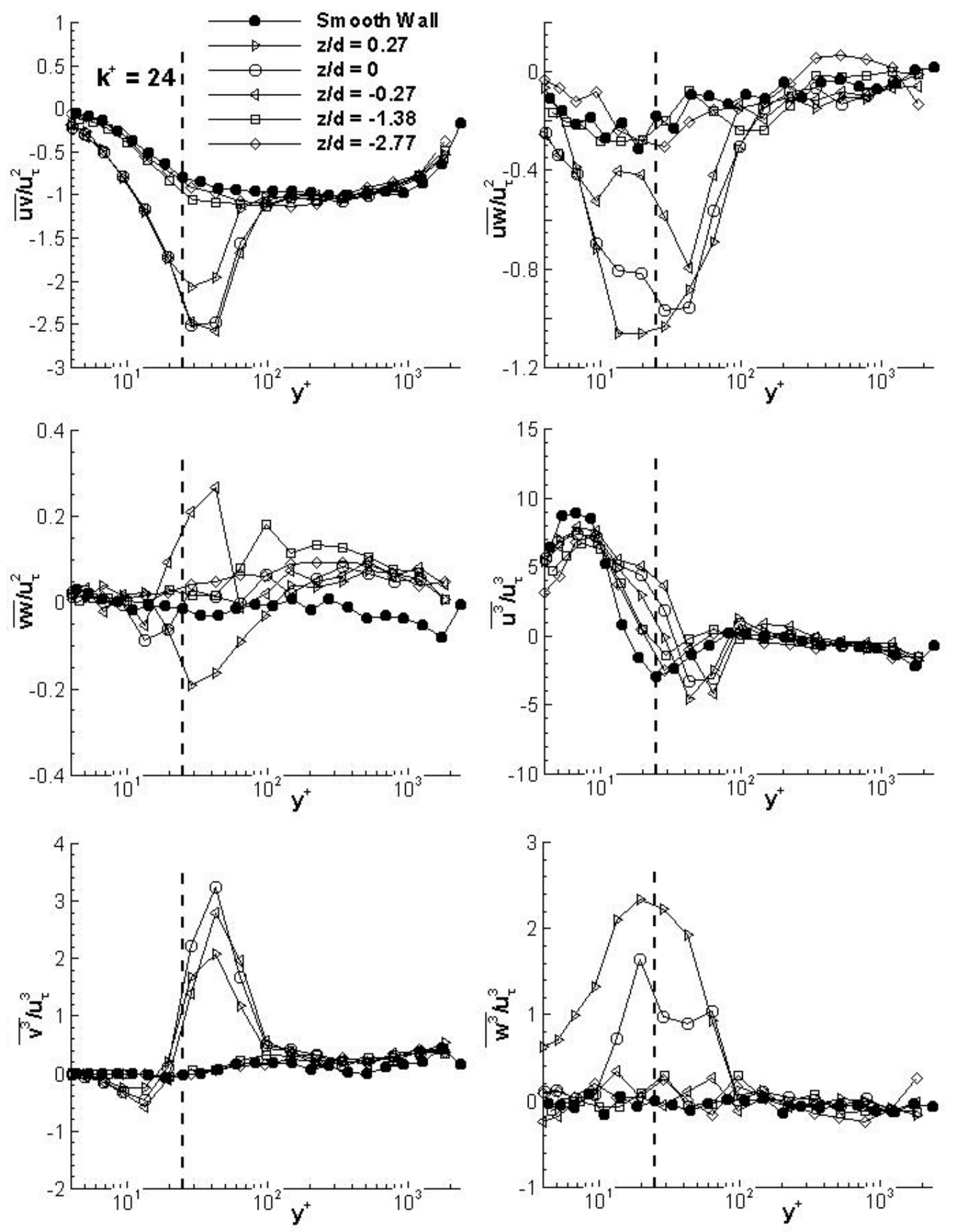

Figure A.26: 0.38 mm Cylinders, straight: Reynolds shear stress and triple product mid-plane plots 

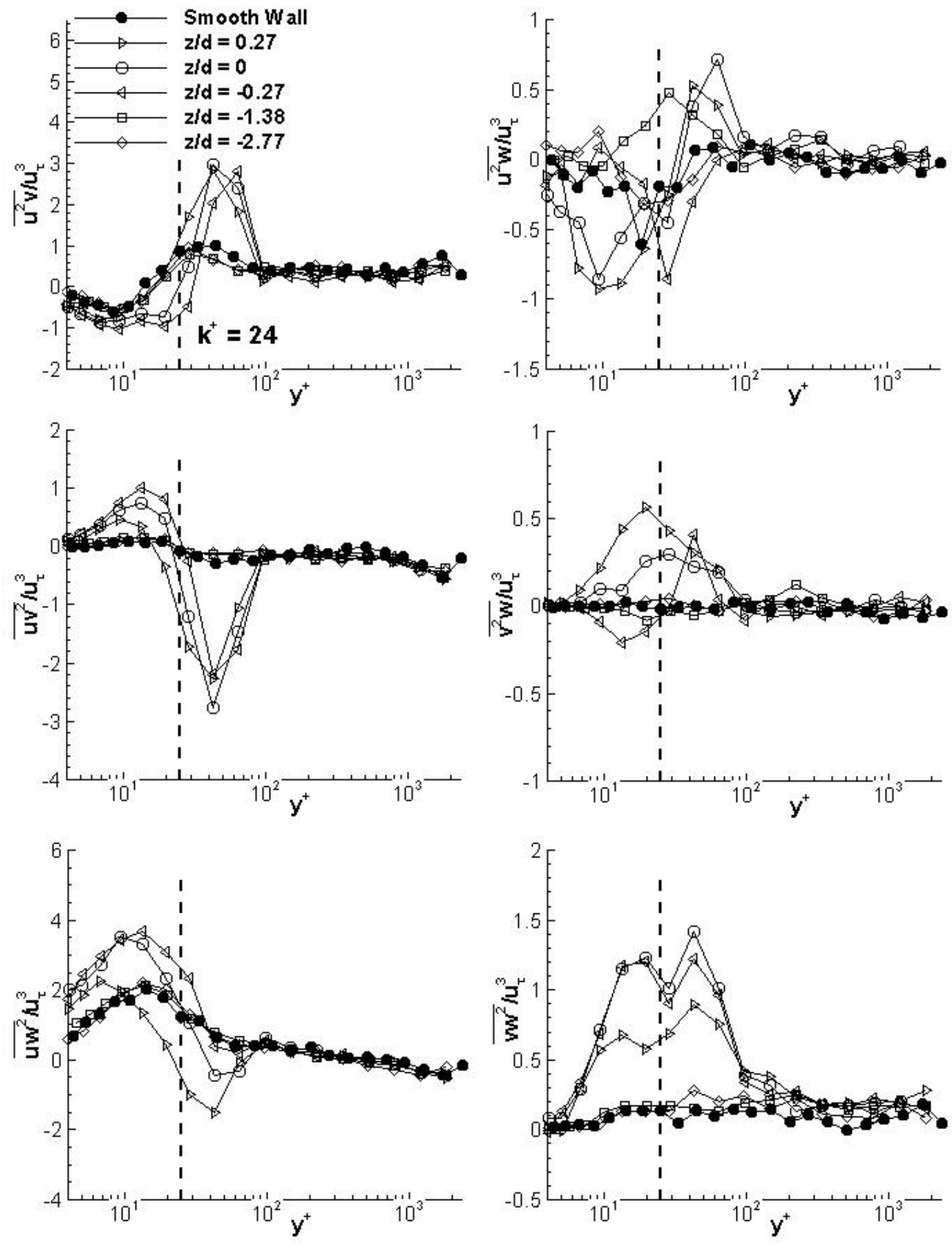

Figure A.27: $0.38 \mathrm{~mm}$ Cylinders, straight: Triple product mid-plane plots (continued) 

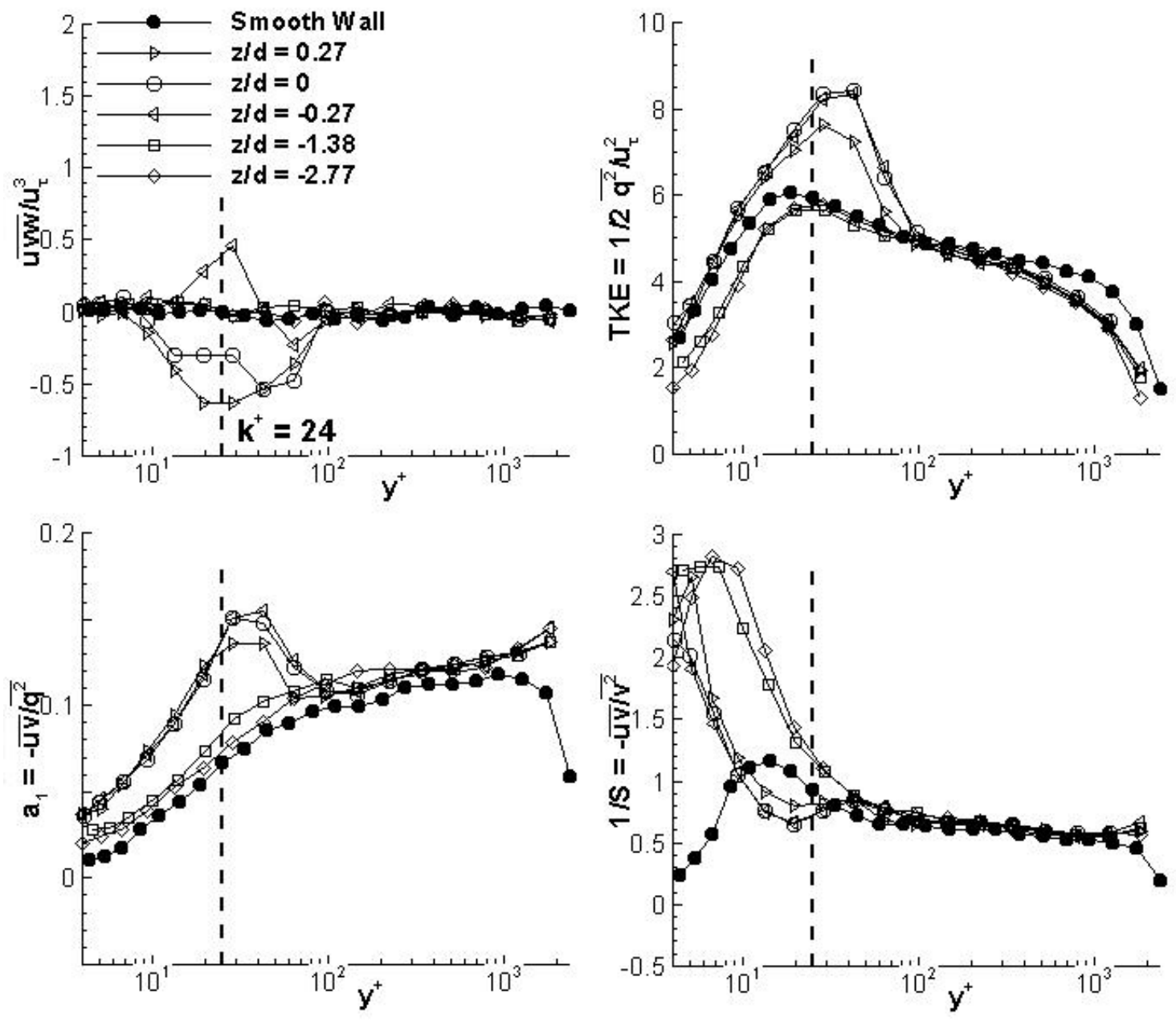

Figure A.28: $0.38 \mathrm{~mm}$ Cylinders, straight: $\overline{u v w} / u_{\tau}^{2}$ triple product, turbulent kinetic energy and structure parameters $a_{1}$ and $1 / S$ mid-plane plots 


\section{A.5 $\quad 0.38 \mathrm{~mm}$ Cylinders, Staggered}
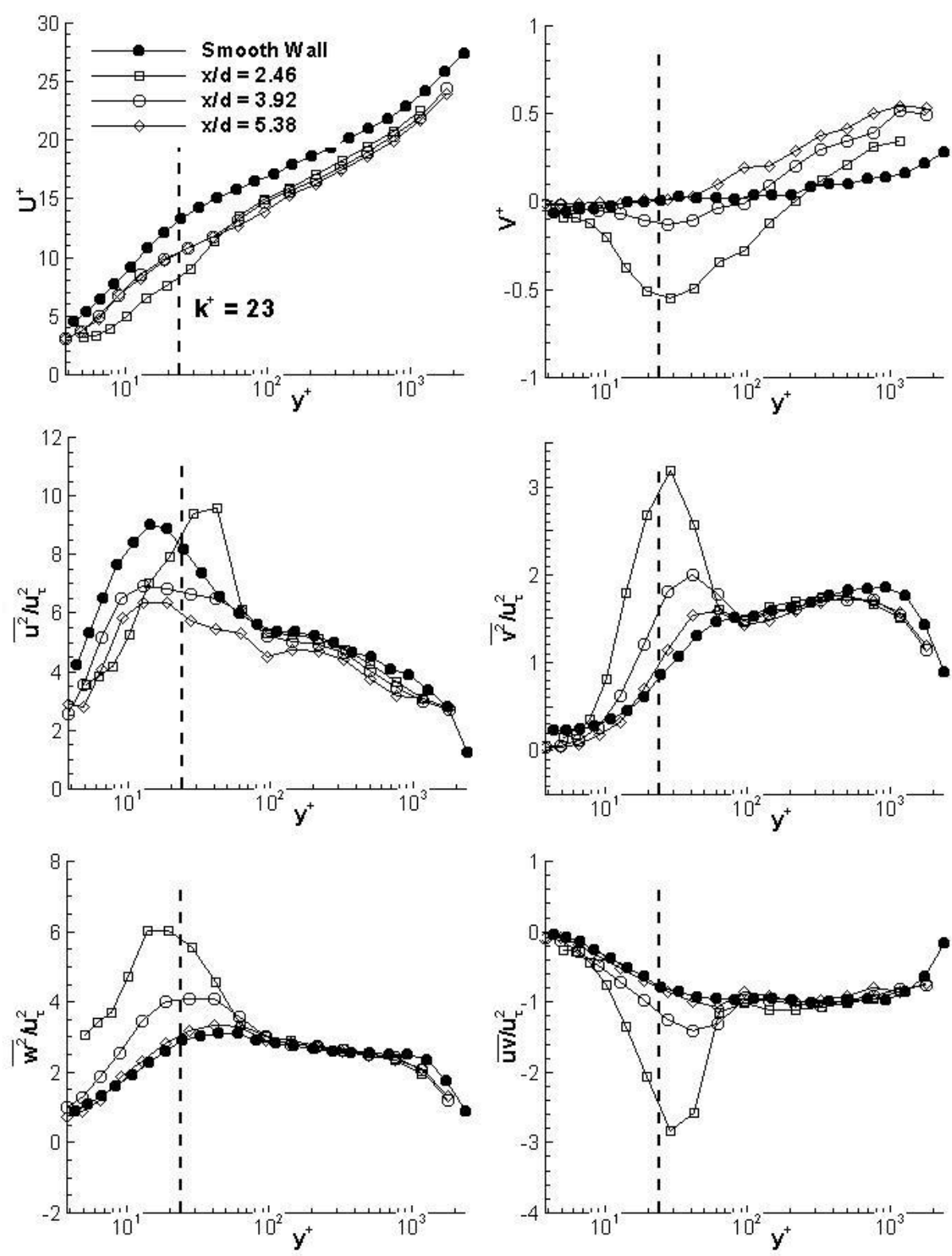

Figure A.29: 0.38 mm Cylinders, staggered: Mean velocity and Reynolds stress centerline plots 

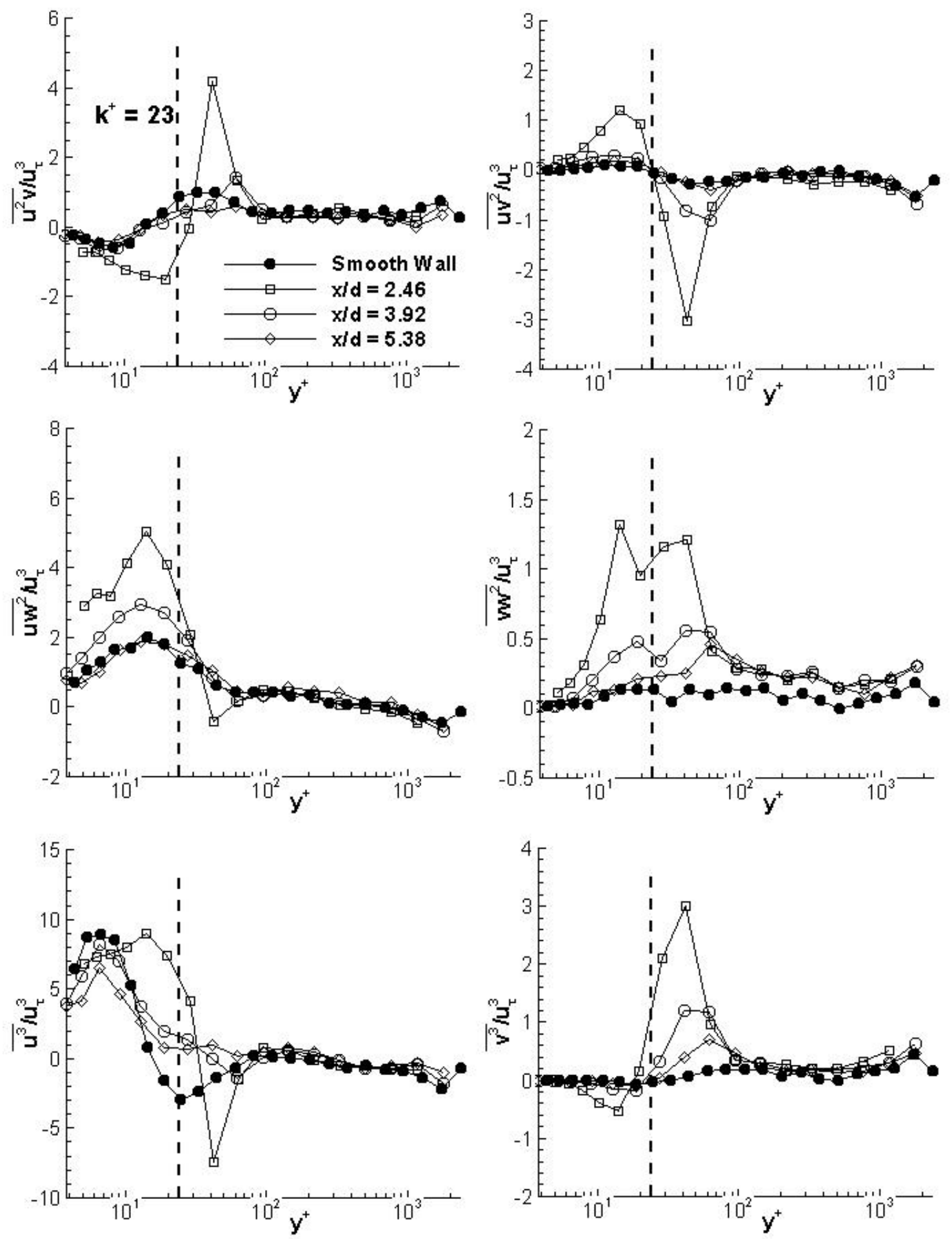

Figure A.30: $0.38 \mathrm{~mm}$ Cylinders, staggered: Triple product centerline plots 

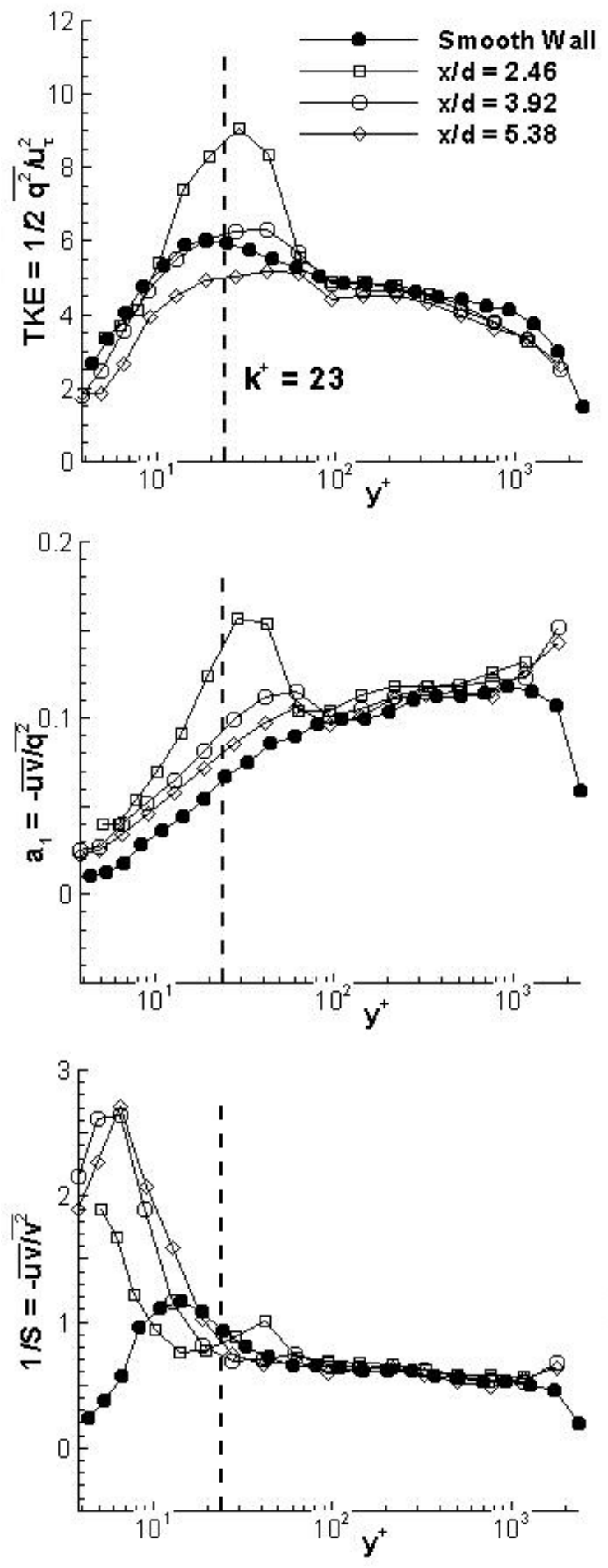

Figure A.31: $0.38 \mathrm{~mm}$ Cylinders, staggered: Turbulent kinetic energy and structure parameters $a_{1}$ and $1 / S$ centerline plots 

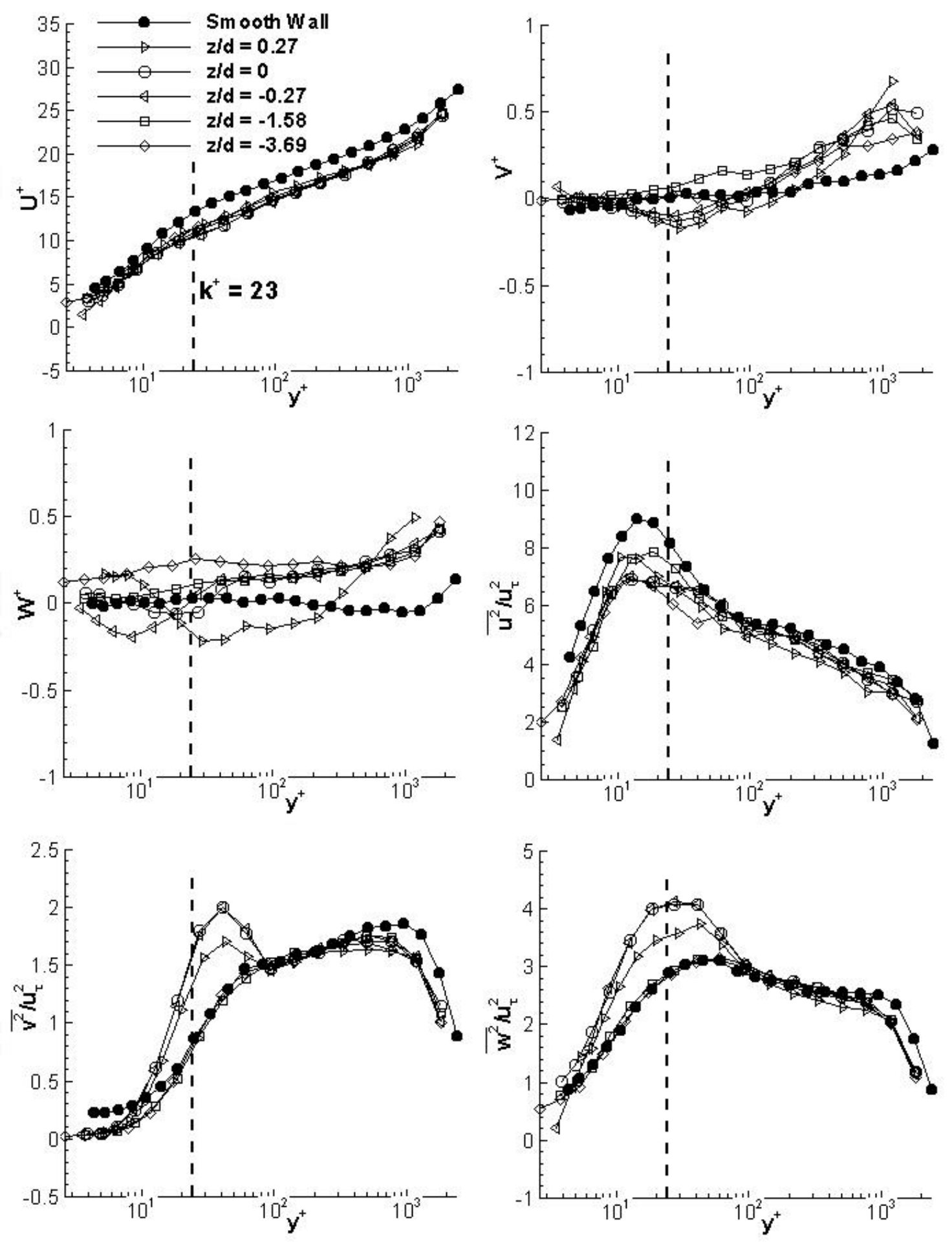

Figure A.32: $0.38 \mathrm{~mm}$ Cylinders, staggered: Mean velocity and Reynolds normal stress mid-plane plots 

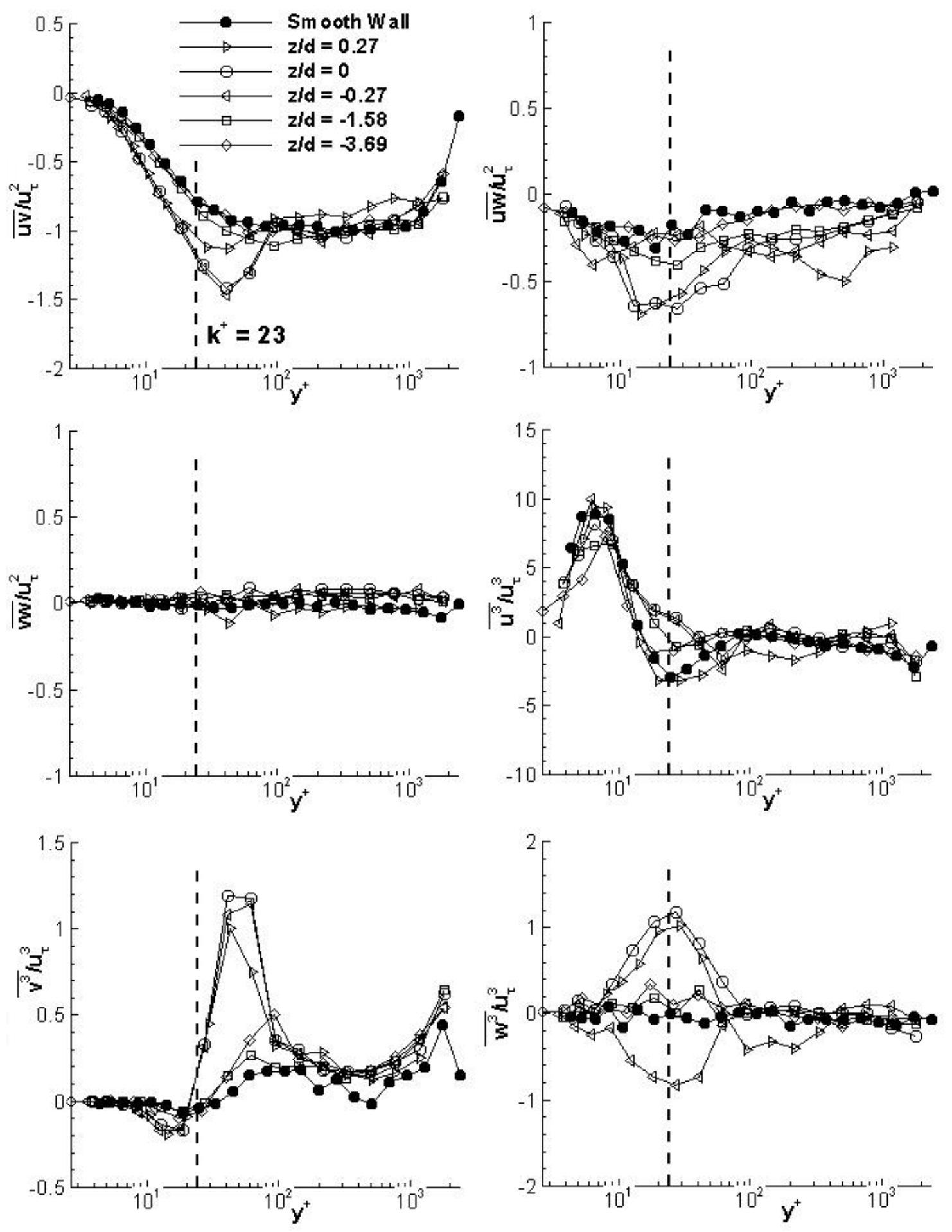

Figure A.33: $0.38 \mathrm{~mm}$ Cylinders, staggered: Reynolds shear stress and triple product mid-plane plots 

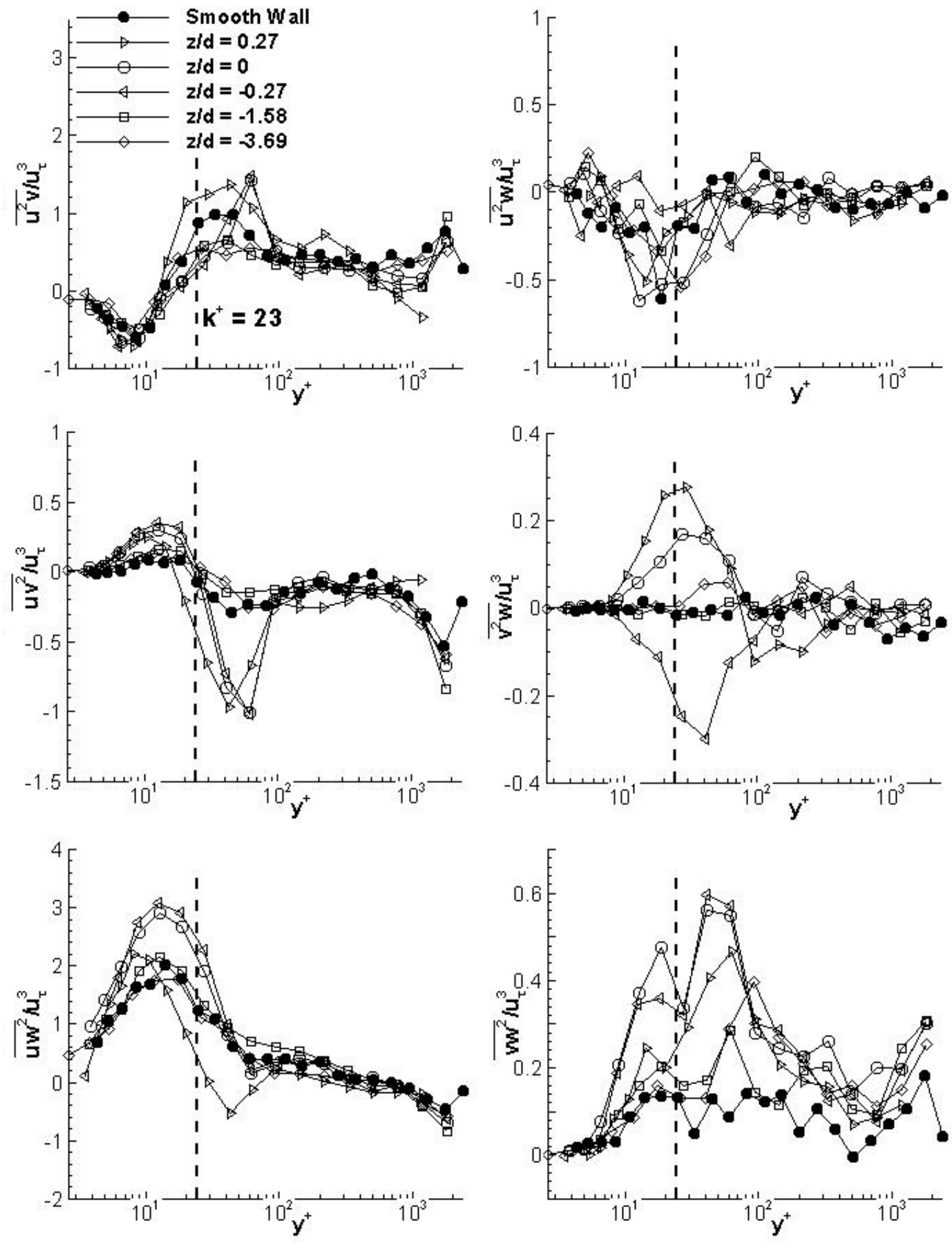

Figure A.34: 0.38 mm Cylinders, staggered: Triple product mid-plane plots (continued) 

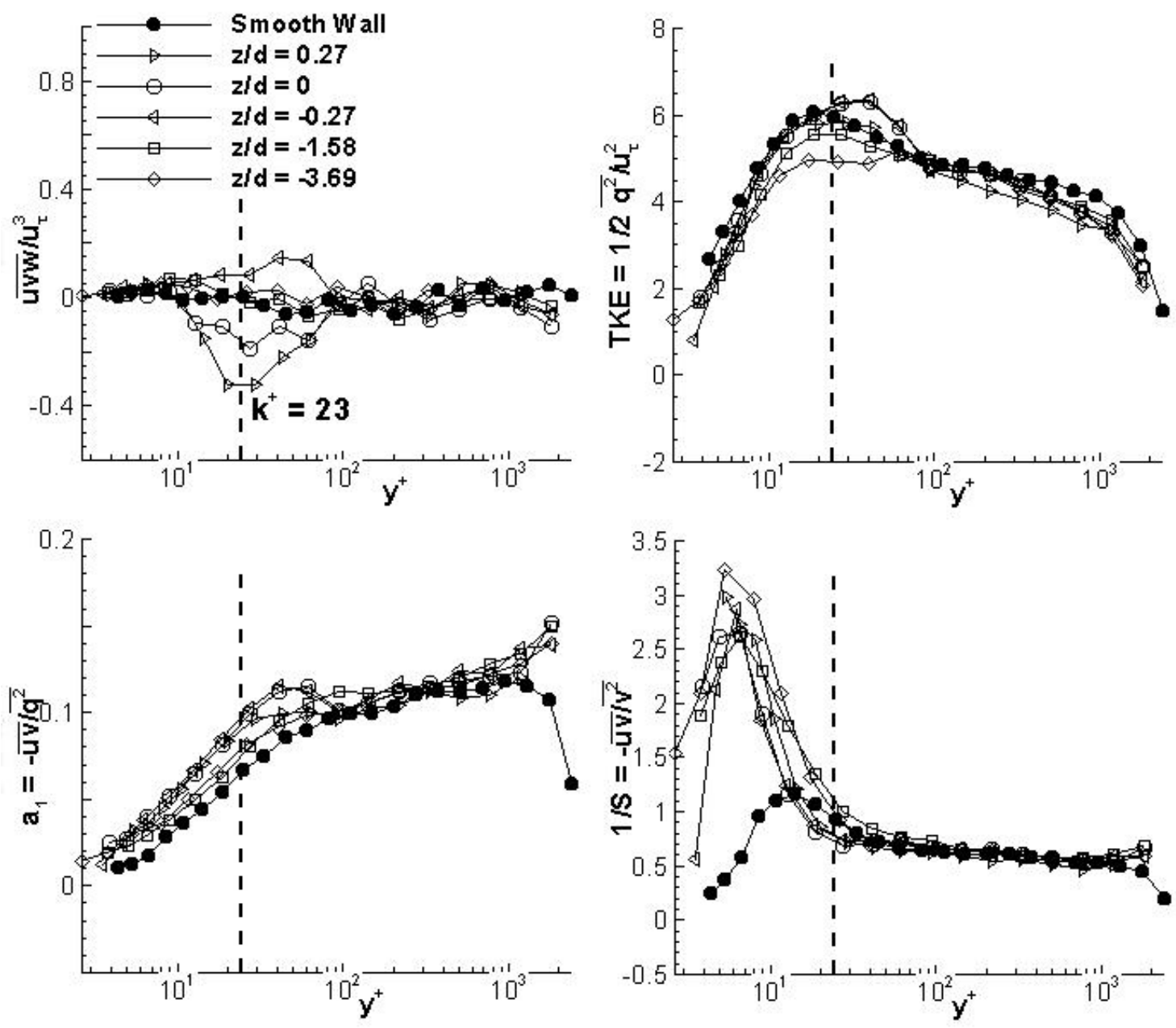

Figure A.35: $0.38 \mathrm{~mm}$ Cylinders, staggered: $\overline{u v w} / u_{\tau}^{2}$ triple product, turbulent kinetic energy and structure parameters $a_{1}$ and $1 / S$ mid-plane plots 


\section{A.6 $\quad 0.76 \mathrm{~mm}$ Cylinders, Straight}
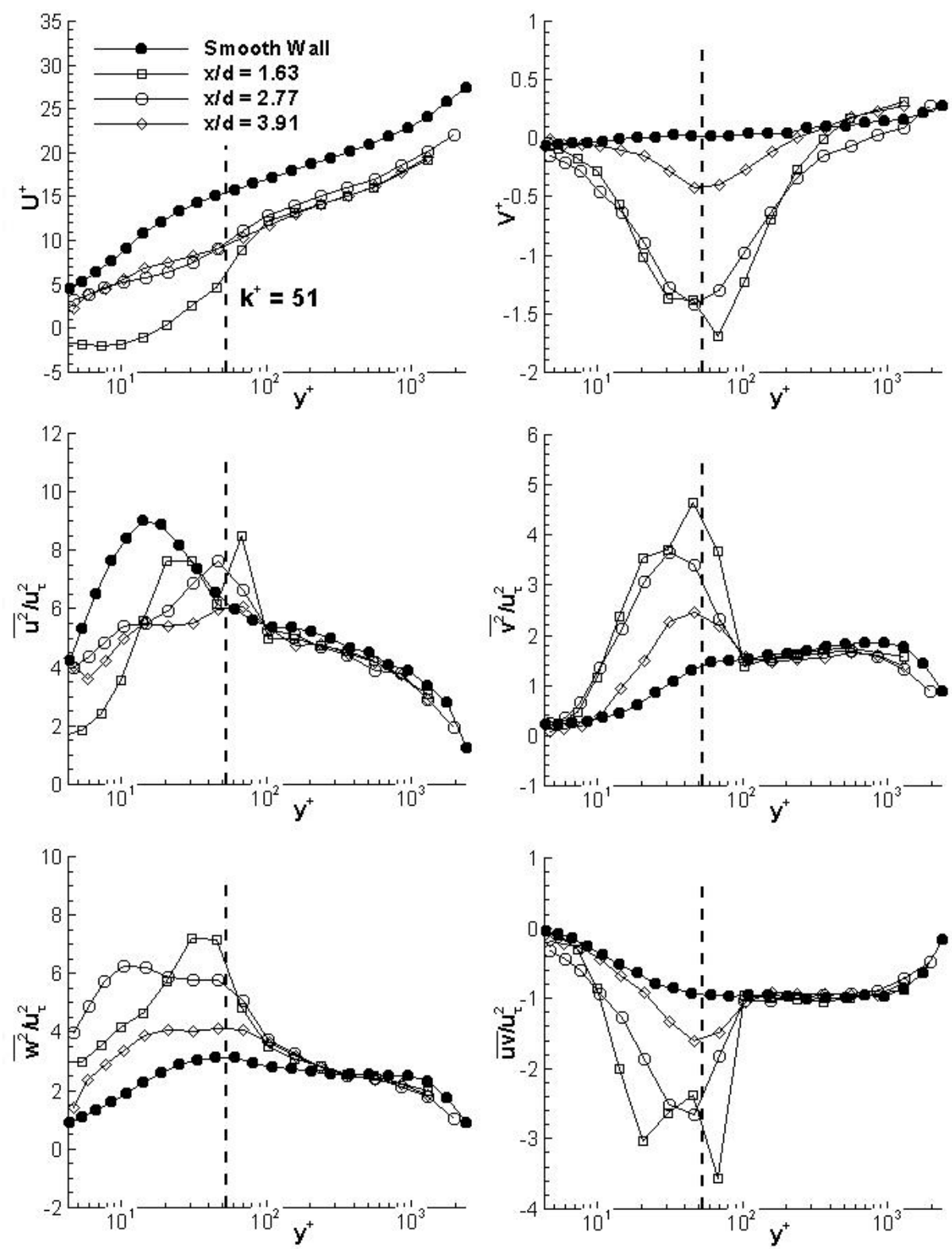

Figure A.36: 0.76 mm Cylinders, straight: Mean velocity and Reynolds stress centerline plots 

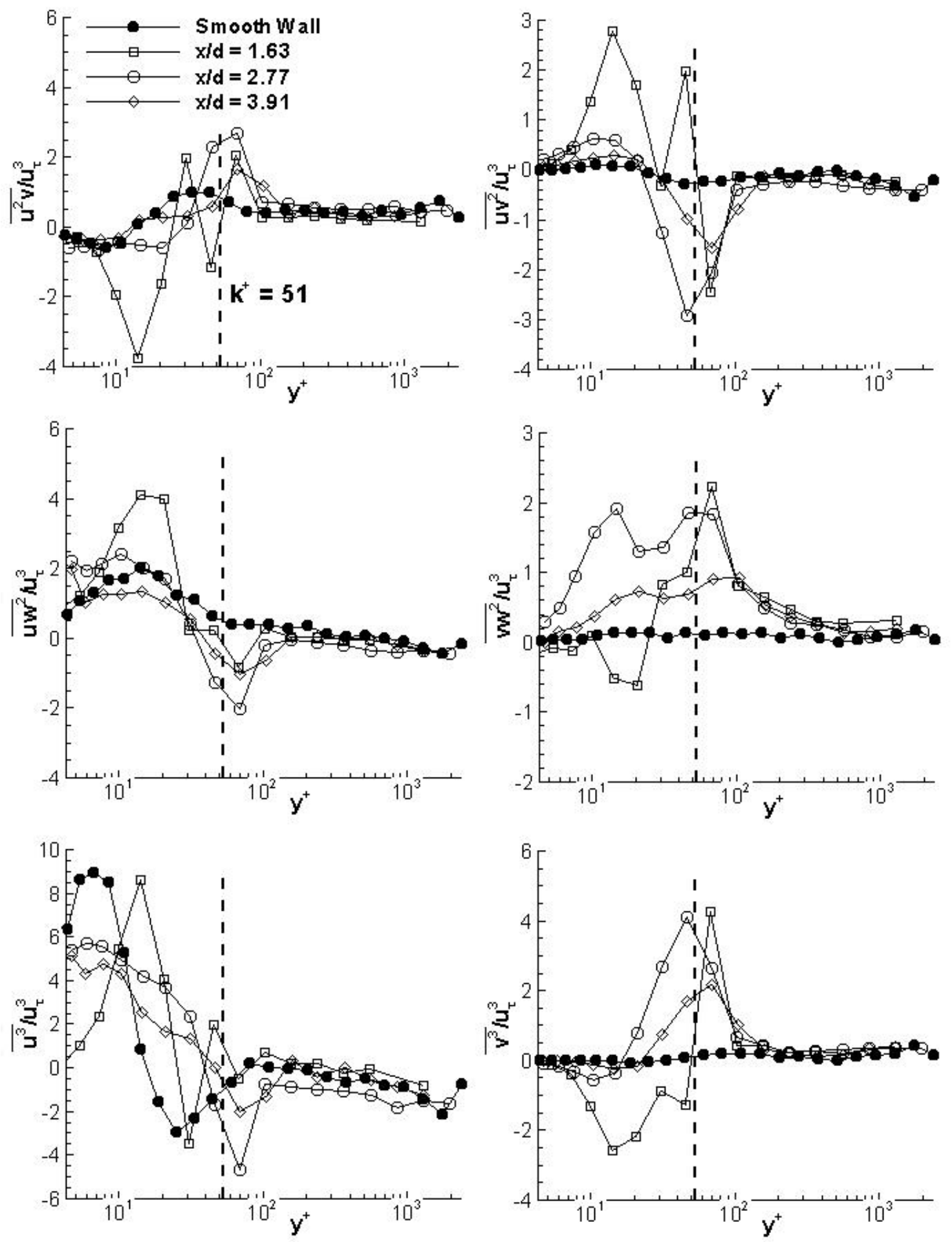

Figure A.37: $0.76 \mathrm{~mm}$ Cylinders, straight: Triple product centerline plots 

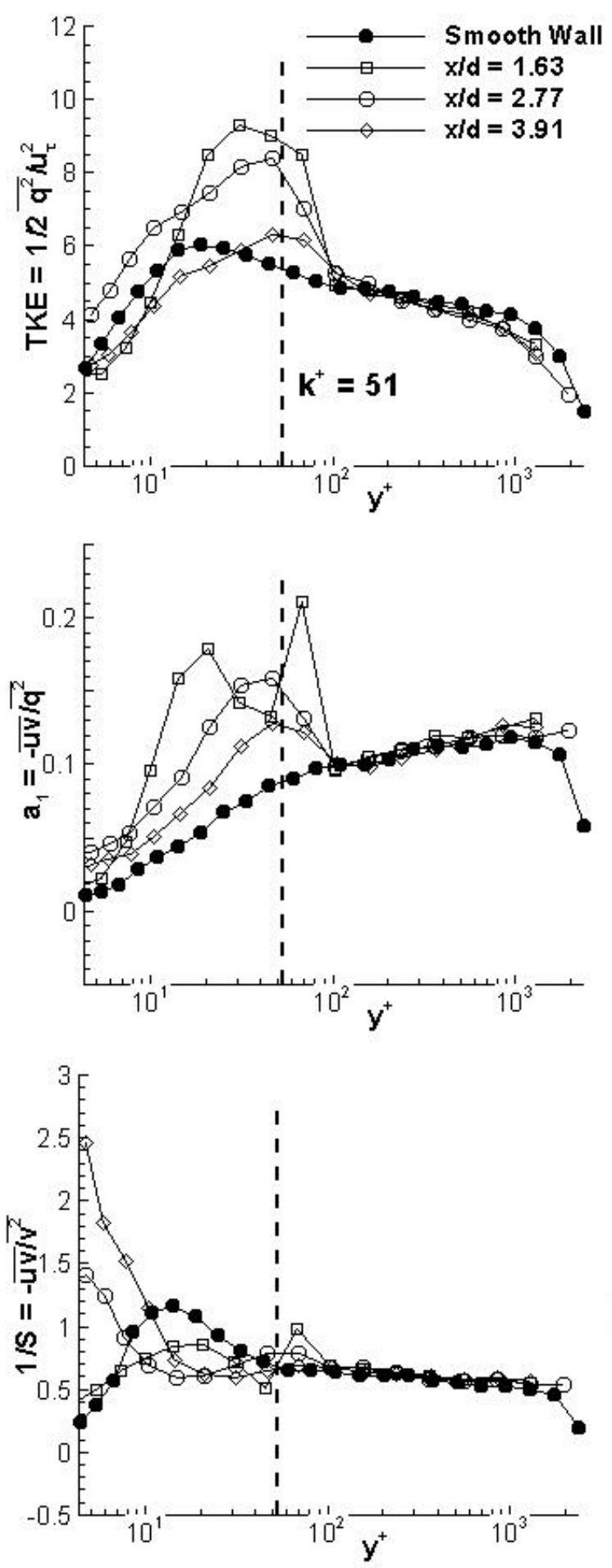

Figure A.38: $0.76 \mathrm{~mm}$ Cylinders, straight: Turbulent kinetic energy and structure parameters $a_{1}$ and $1 / S$ centerline plots 

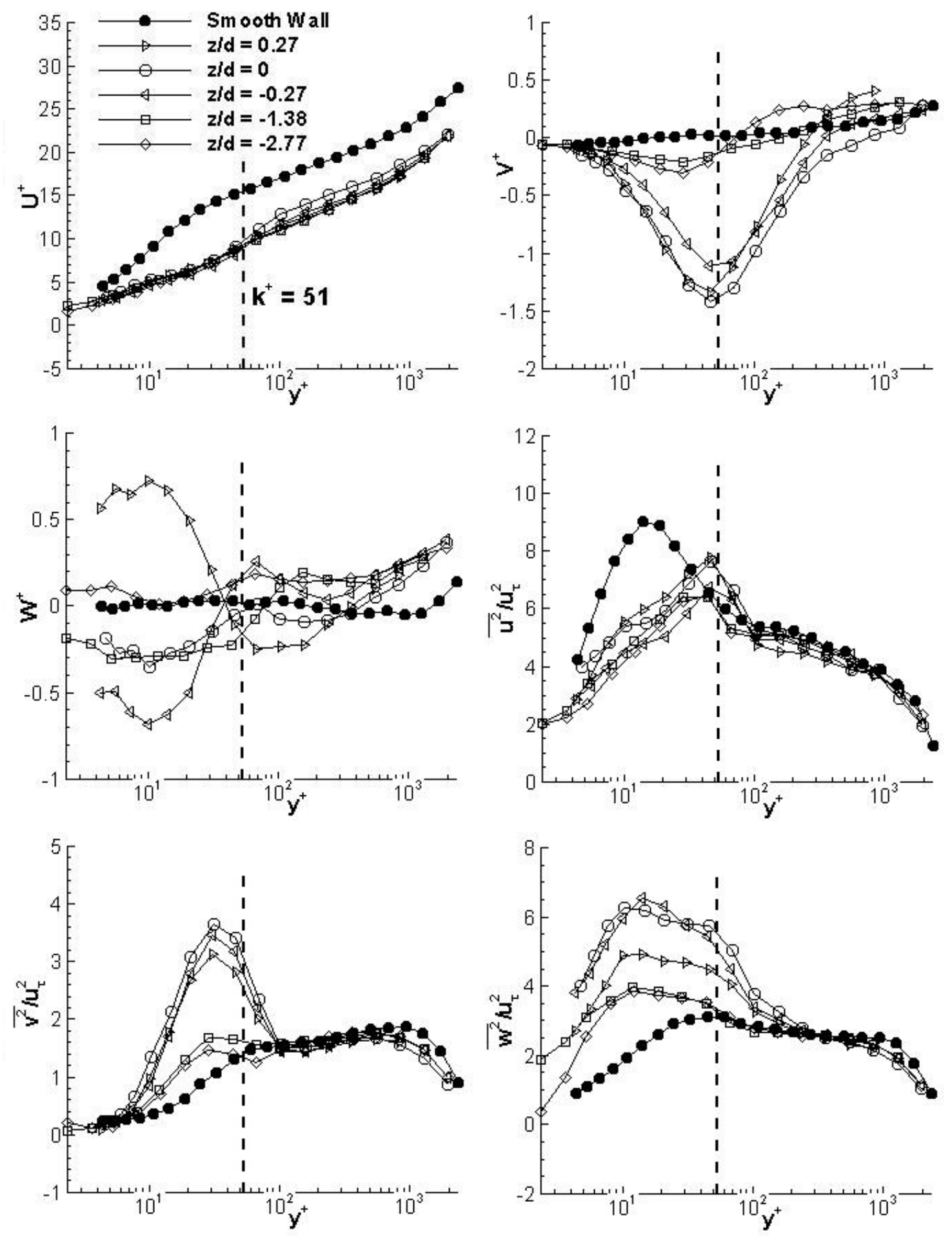

Figure A.39: $0.76 \mathrm{~mm}$ Cylinders, straight: Mean velocity and Reynolds normal stress mid-plane plots 

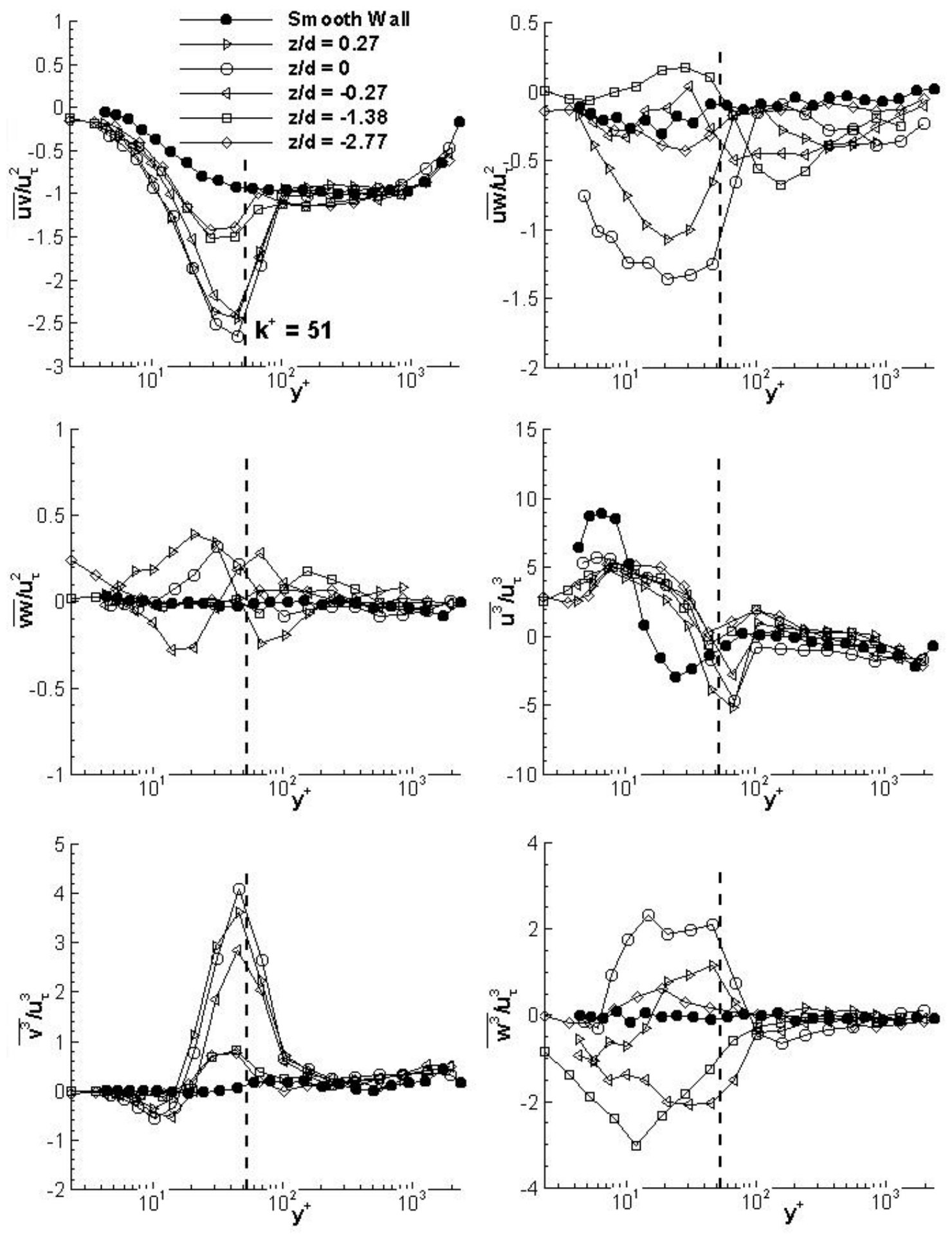

Figure A.40: $0.76 \mathrm{~mm}$ Cylinders, straight: Reynolds shear stress and triple product mid-plane plots 

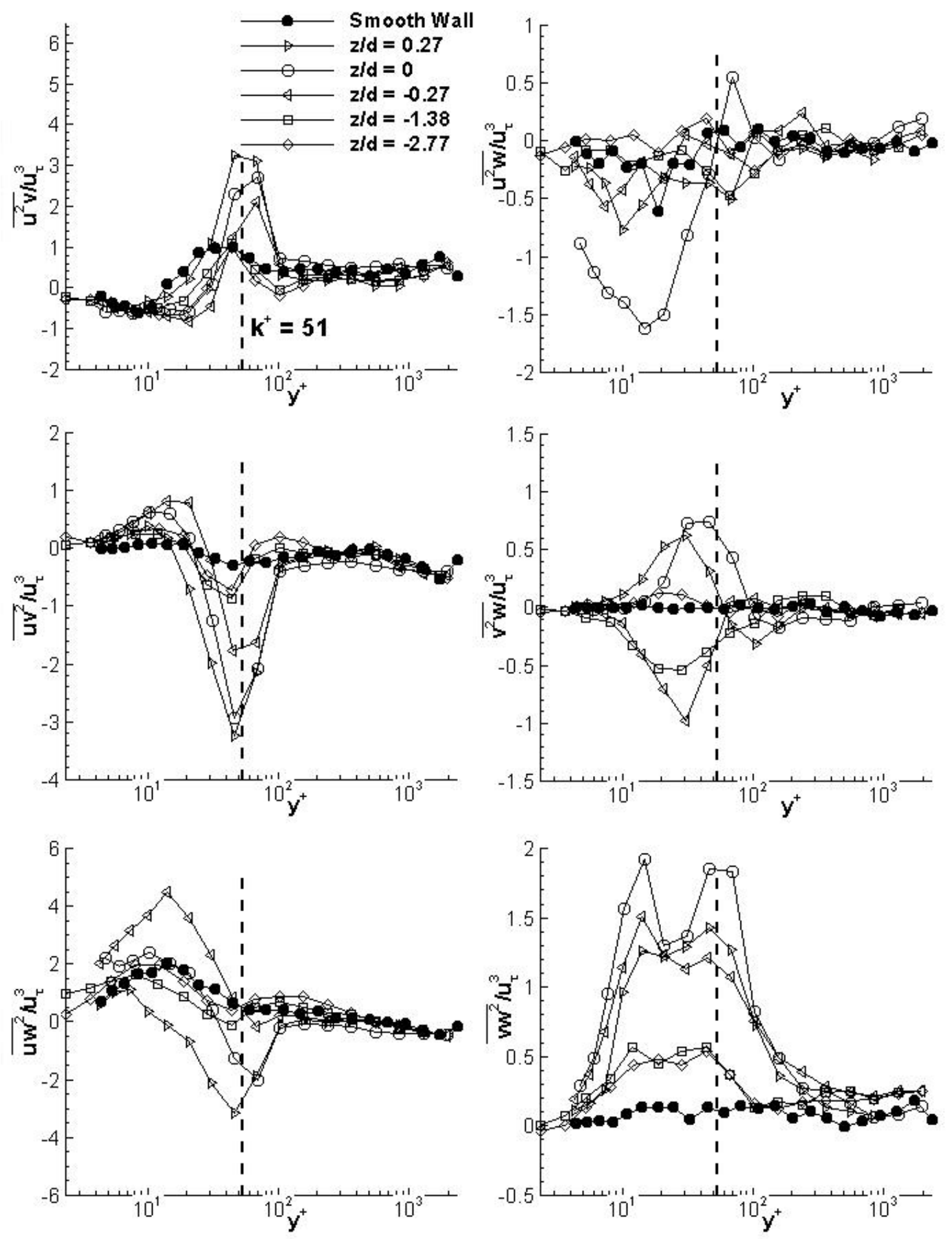

Figure A.41: $0.76 \mathrm{~mm}$ Cylinders, straight: Triple product mid-plane plots (continued) 

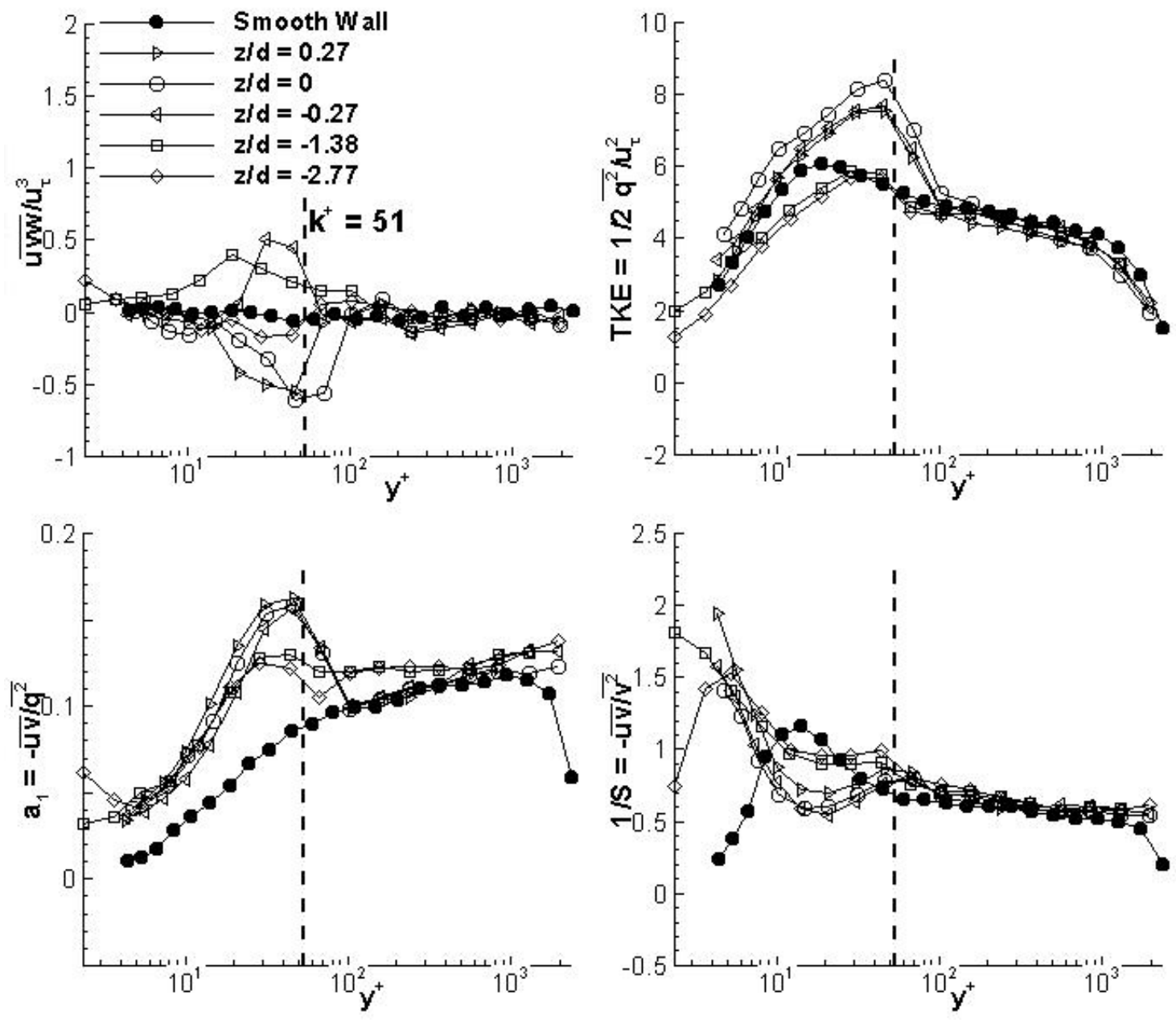

Figure A.42: $0.76 \mathrm{~mm}$ Cylinders, straight: $\overline{u v w} / u_{\tau}^{2}$ triple product, turbulent kinetic energy and structure parameters $a_{1}$ and $1 / S$ mid-plane plots 


\section{A.7 $\quad 0.76 \mathrm{~mm}$ Cylinders, Staggered}
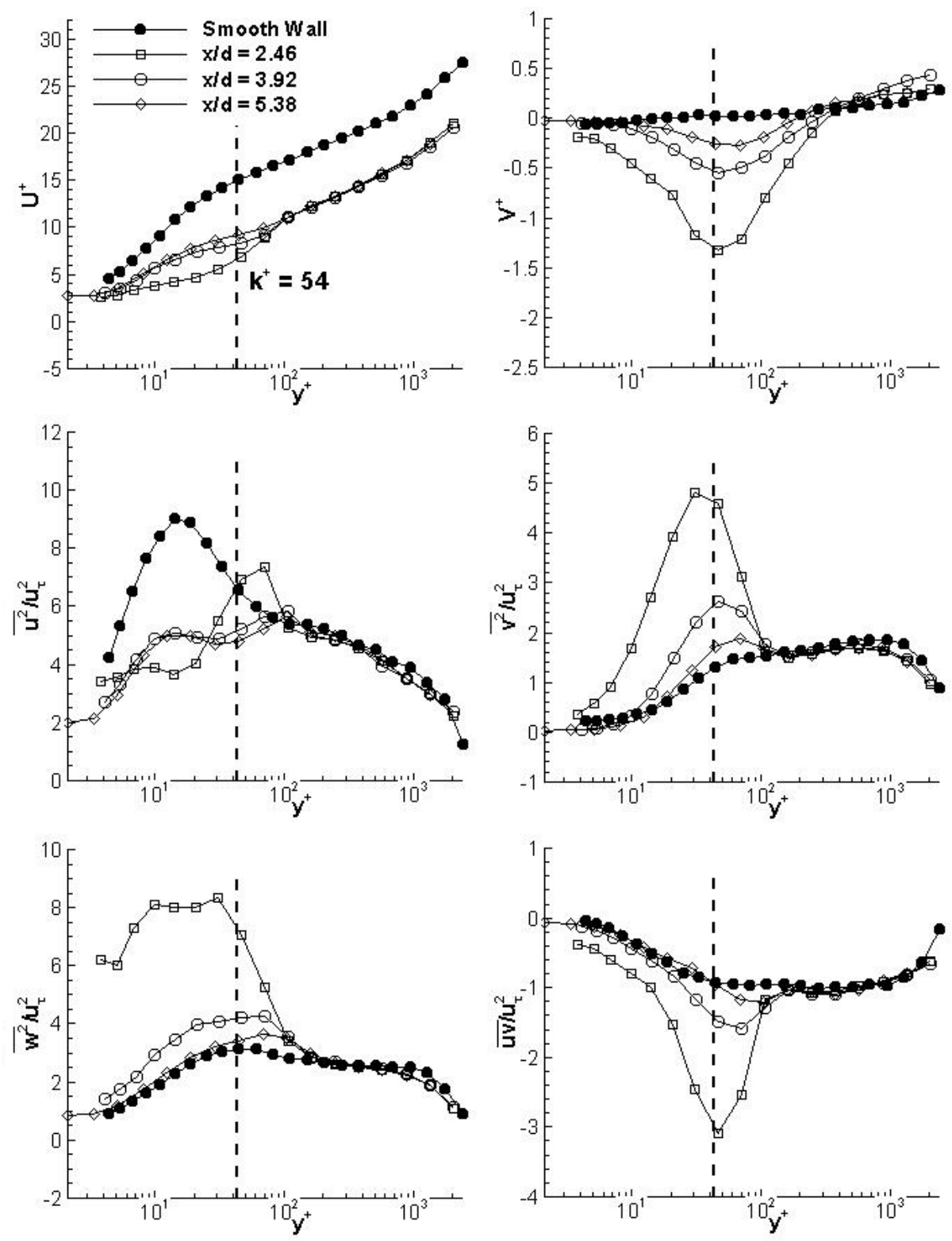

Figure A.43: $0.76 \mathrm{~mm}$ Cylinders, staggered: Mean velocity and Reynolds stress centerline plots 

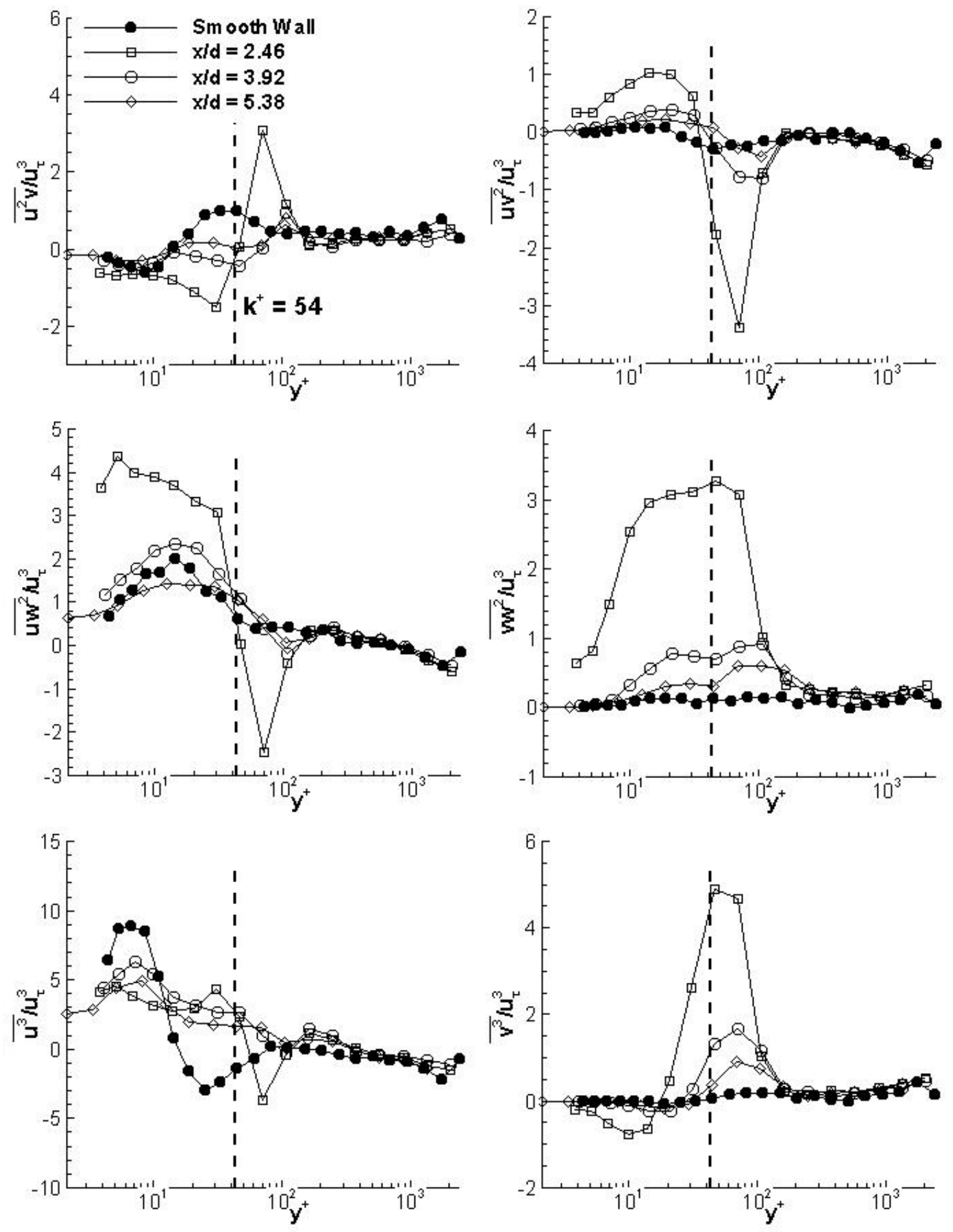

Figure A.44: $0.76 \mathrm{~mm}$ Cylinders, staggered: Triple product centerline plots 

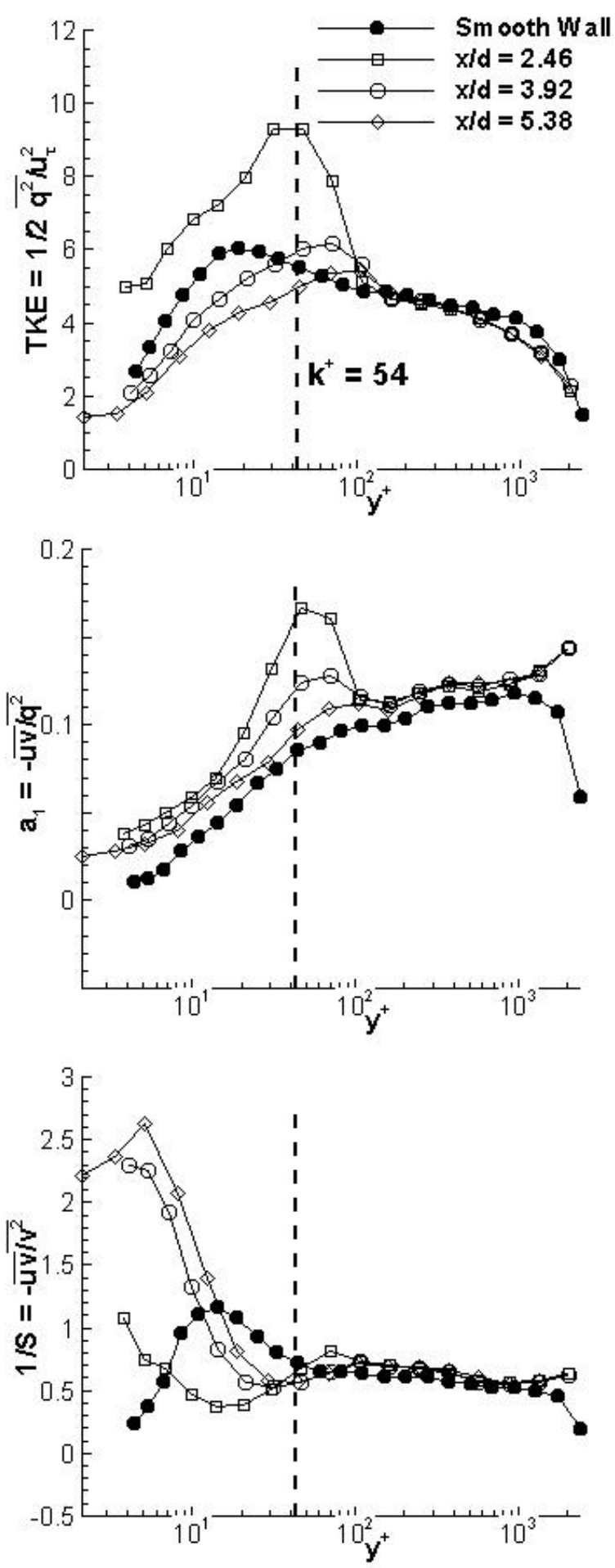

Figure A.45: $0.76 \mathrm{~mm}$ Cylinders, staggered: Turbulent kinetic energy and structure parameters $a_{1}$ and $1 / S$ centerline plots 

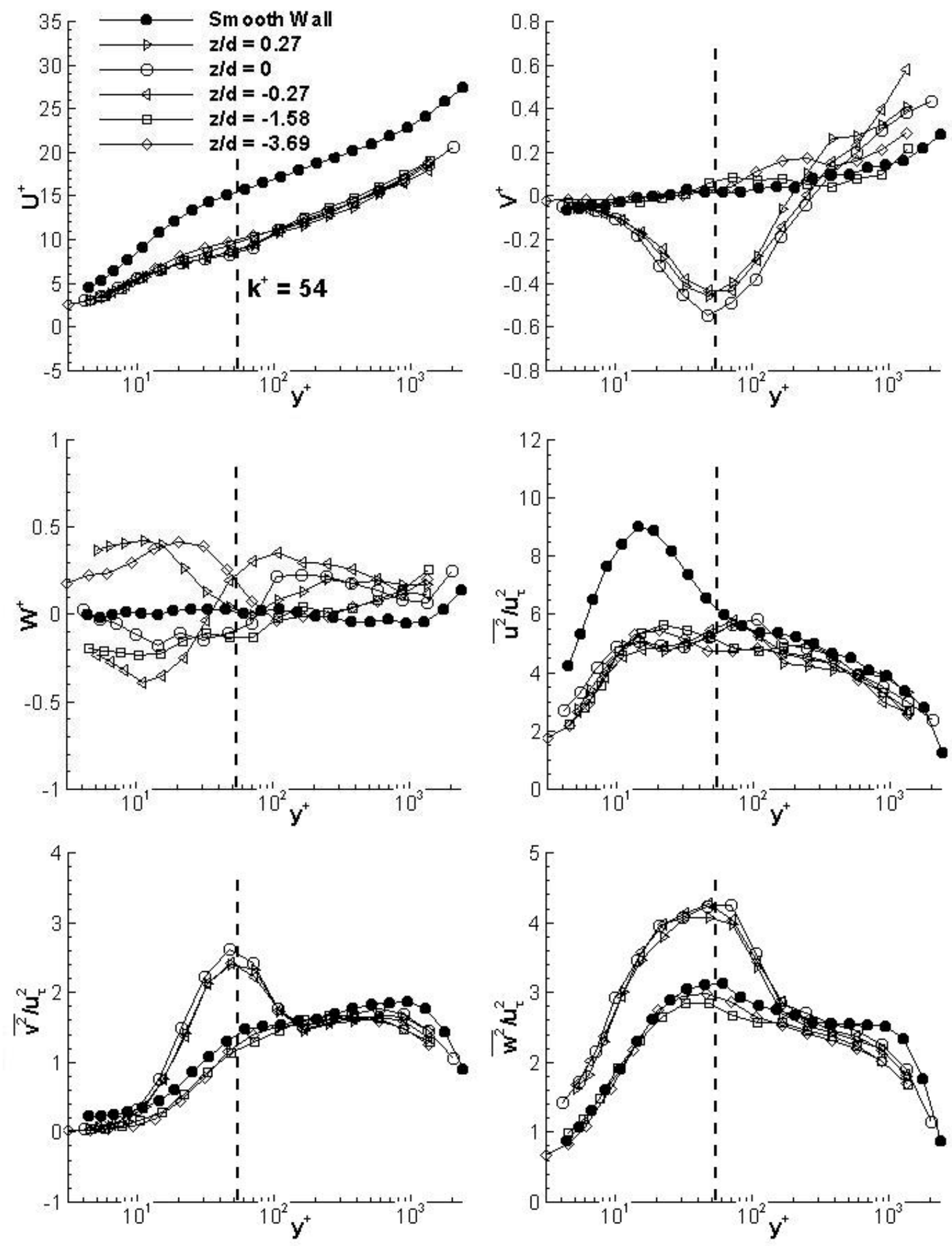

Figure A.46: $0.76 \mathrm{~mm}$ Cylinders, staggered: Mean velocity and Reynolds normal stress mid-plane plots 

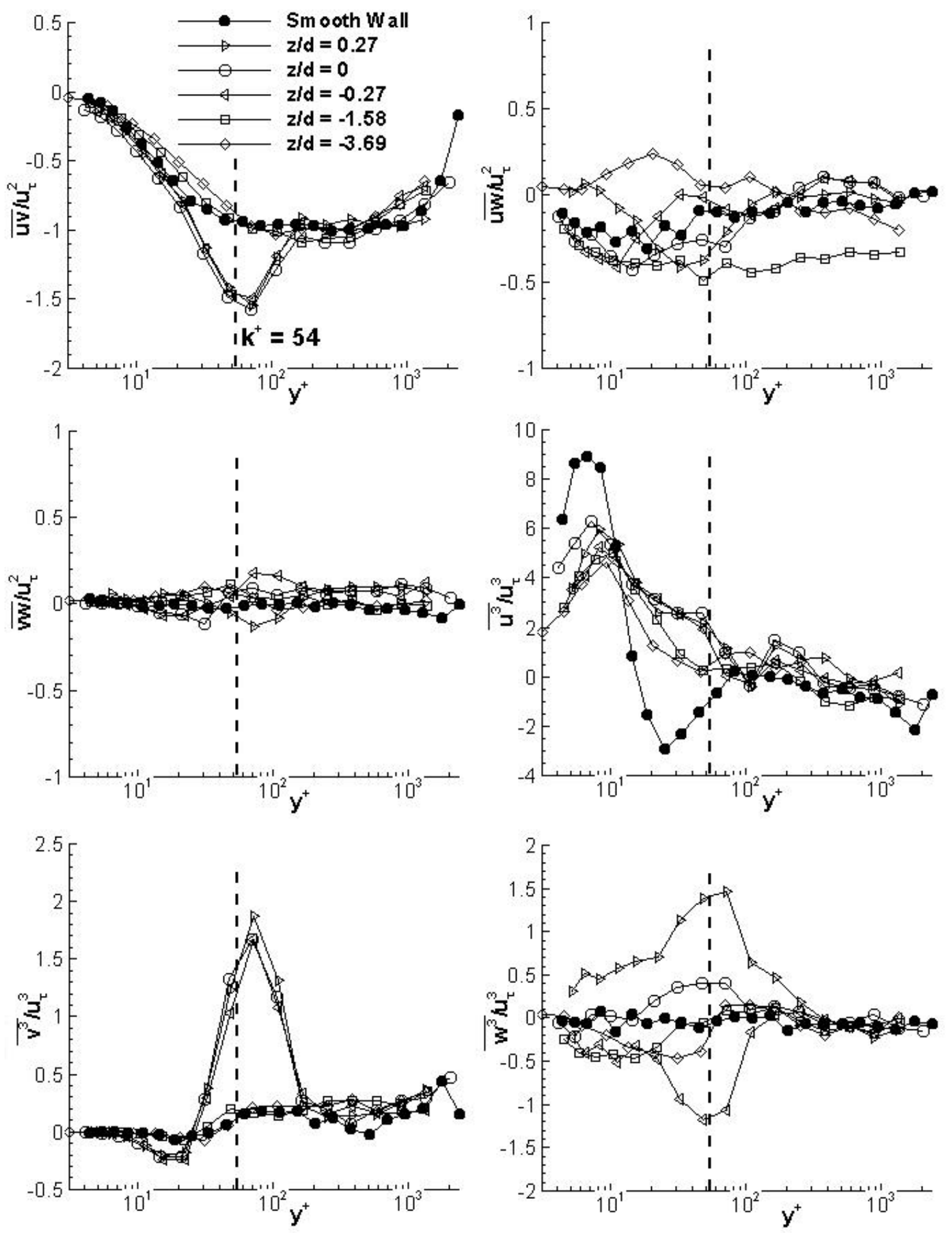

Figure A.47: 0.76 mm Cylinders, staggered: Reynolds shear stress and triple product mid-plane plots 

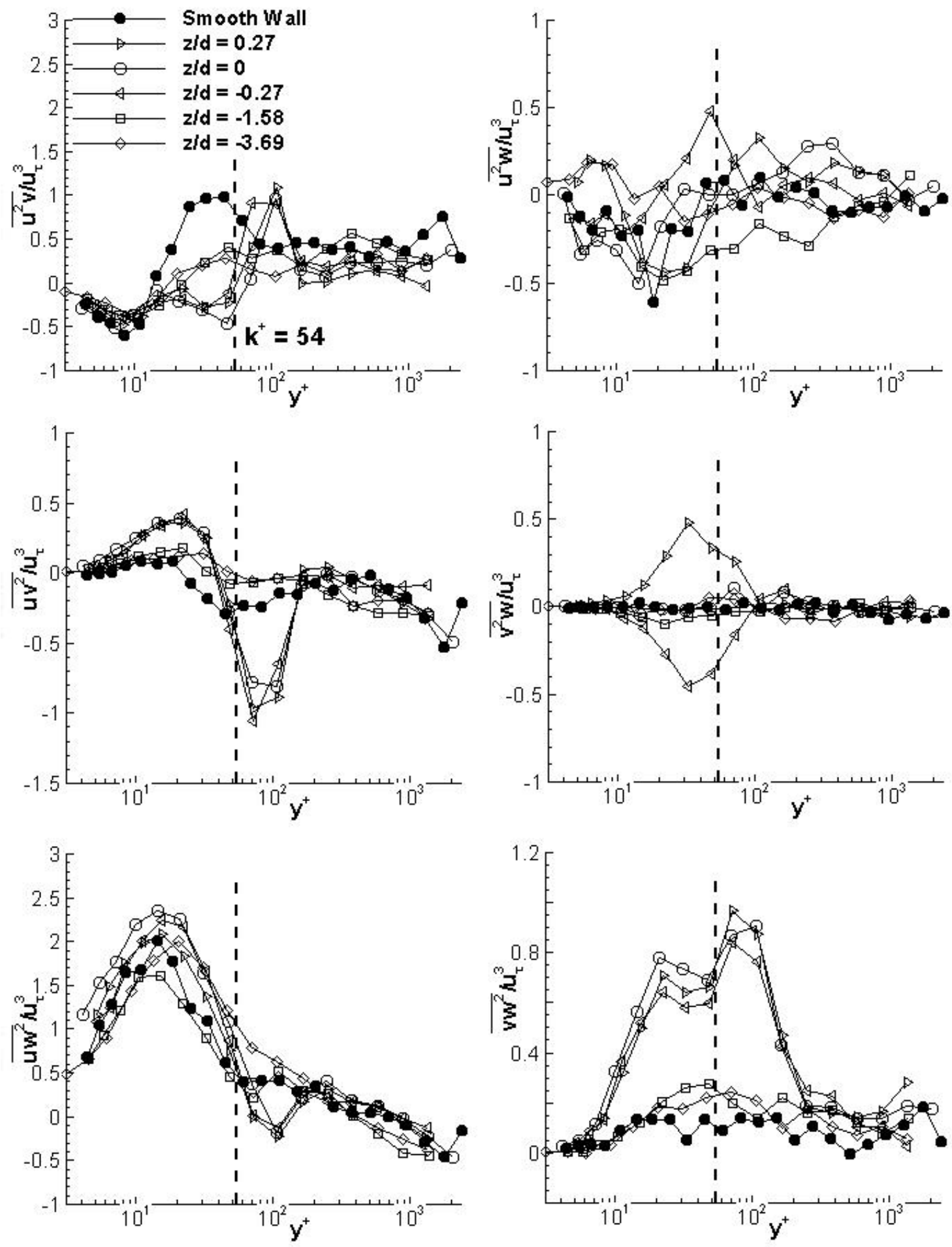

Figure A.48: $0.76 \mathrm{~mm}$ Cylinders, staggered: Triple product mid-plane plots (continued) 

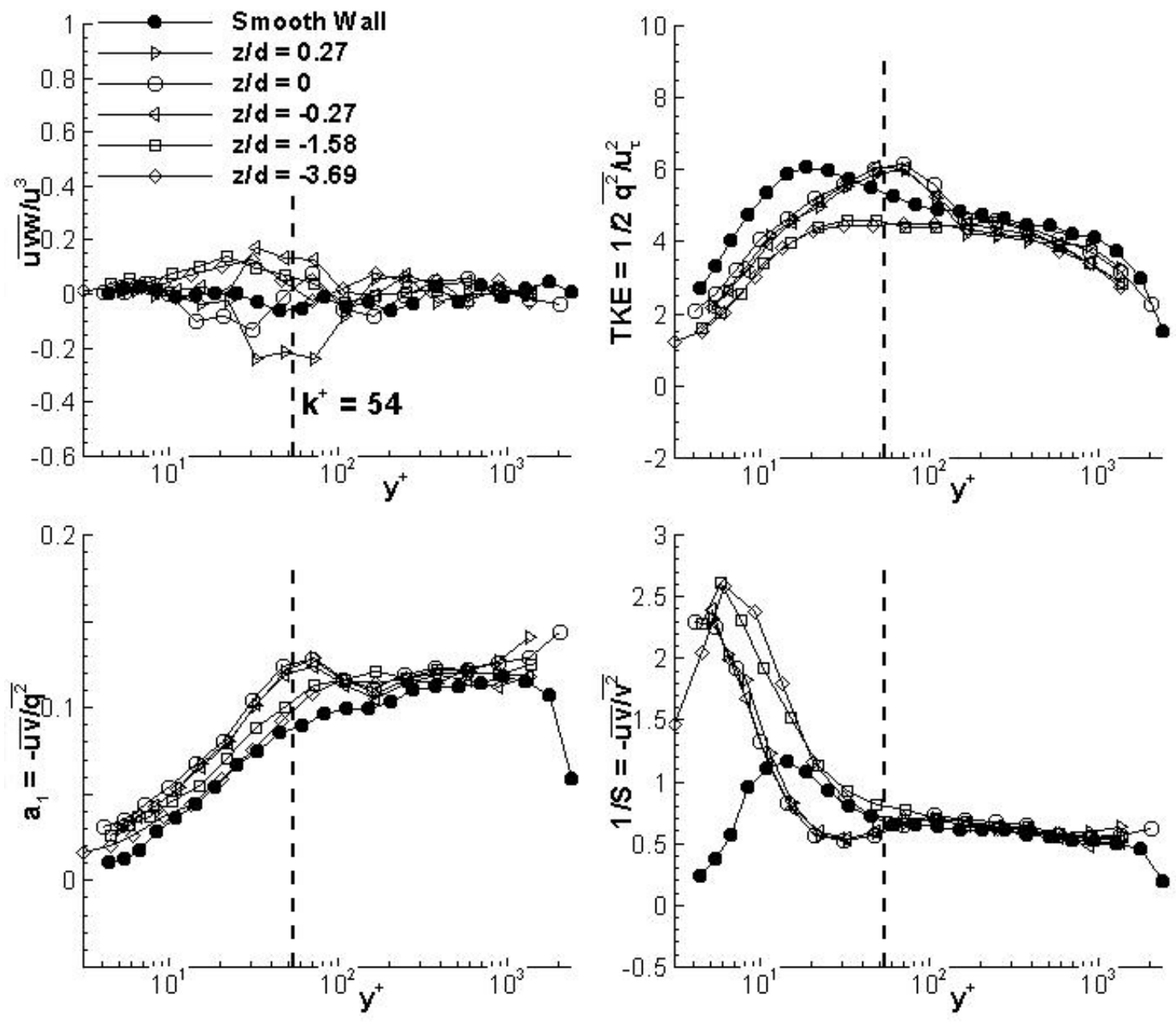

Figure A.49: $0.76 \mathrm{~mm}$ Cylinders, staggered: $\overline{u v w} / u_{\tau}^{2}$ triple product, turbulent kinetic energy and structure parameters $a_{1}$ and $1 / S$ mid-plane plots 


\section{A.8 $1.52 \mathrm{~mm}$ Cylinders, Straight}
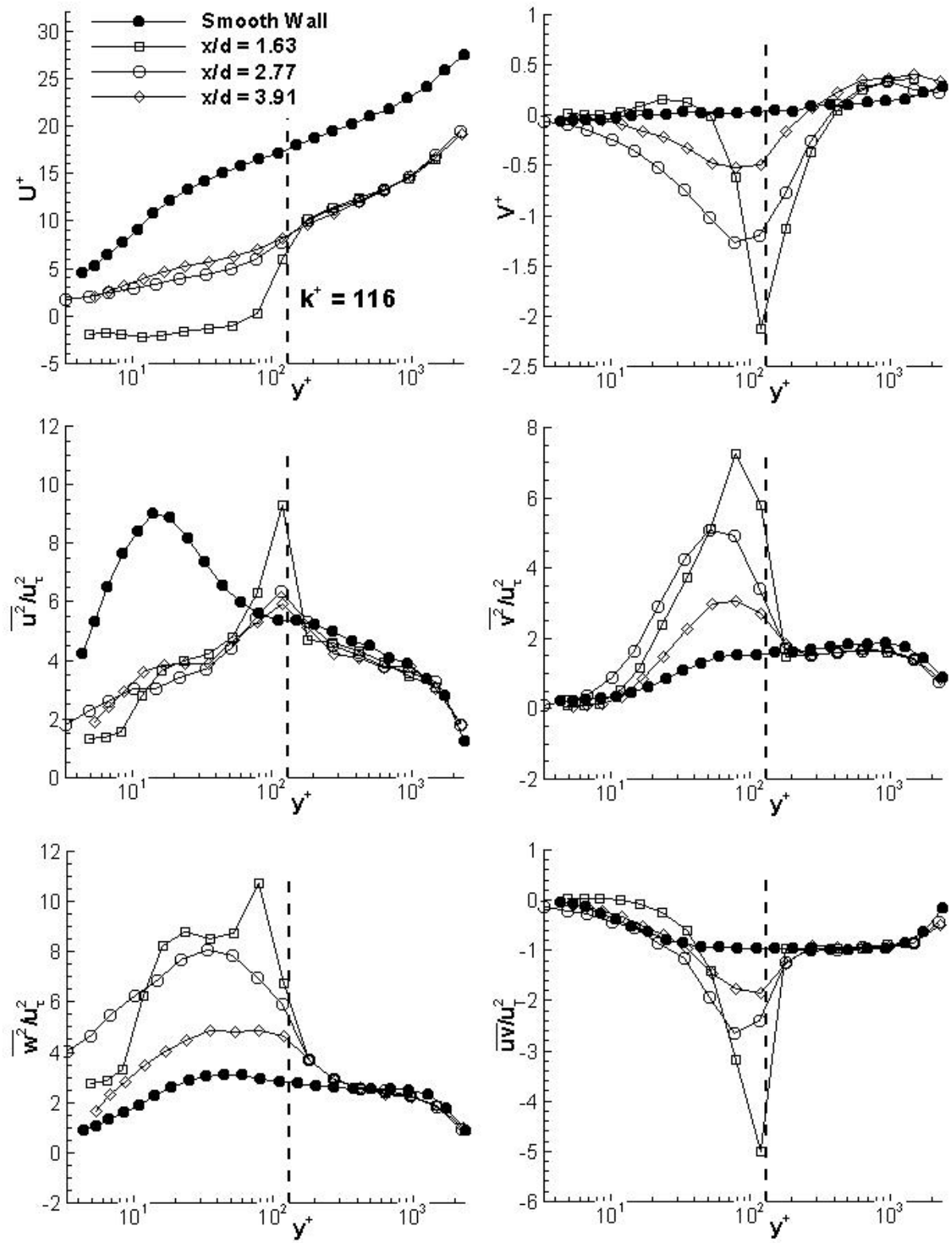

Figure A.50: 1.52 mm Cylinders, straight: Mean velocity and Reynolds stress centerline plots 

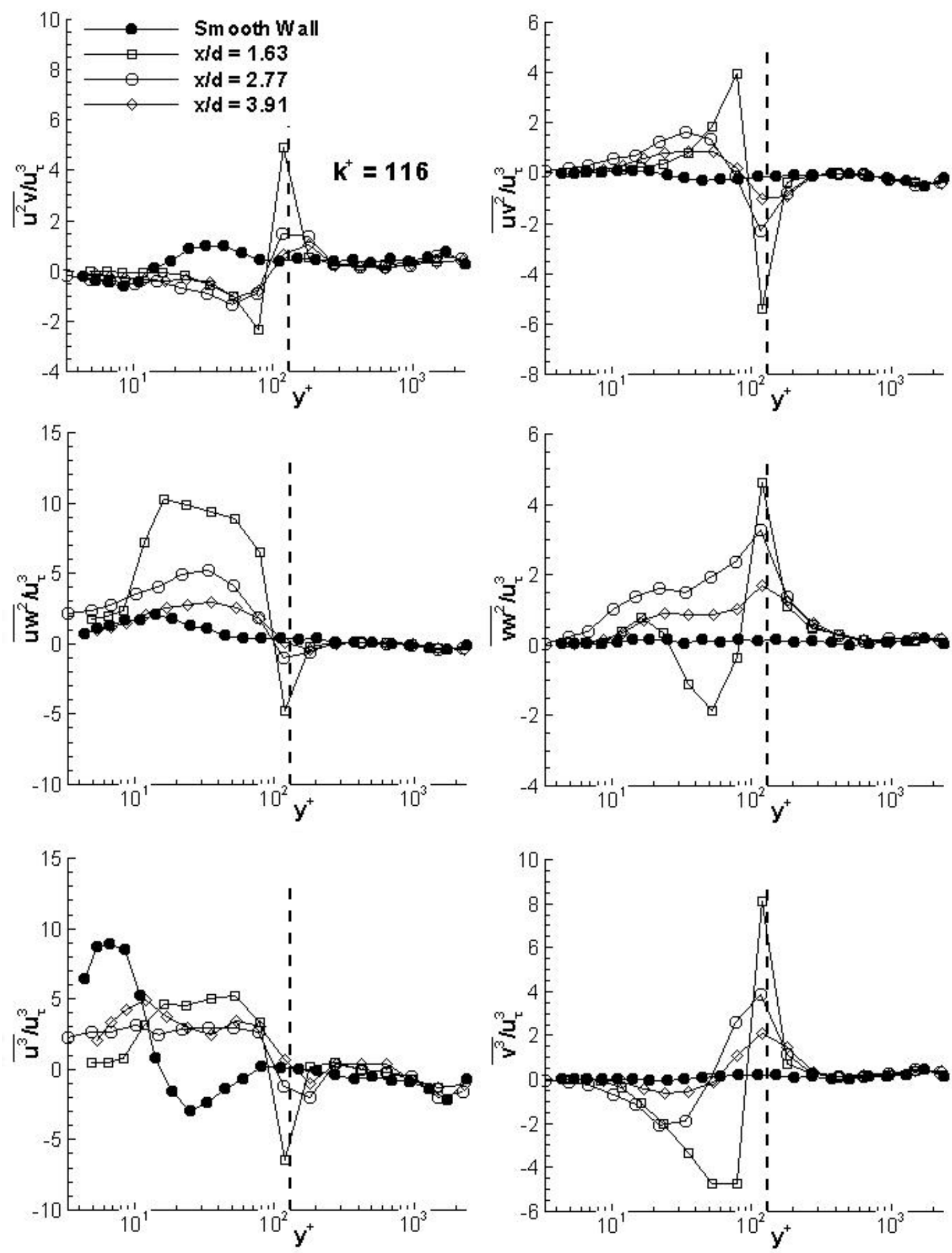

Figure A.51: $1.52 \mathrm{~mm}$ Cylinders, straight: Triple product centerline plots 

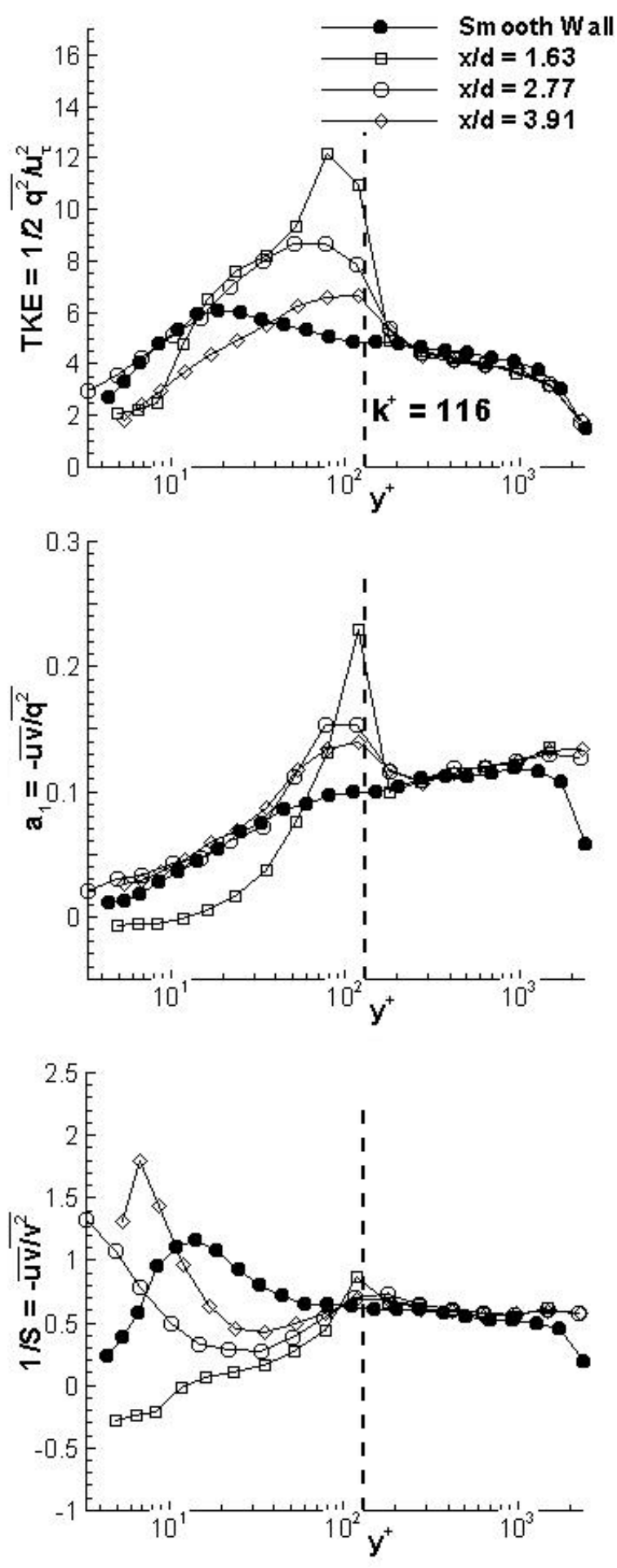

Figure A.52: $1.52 \mathrm{~mm}$ Cylinders, straight: Turbulent kinetic energy and structure parameters $a_{1}$ and $1 / S$ centerline plots 

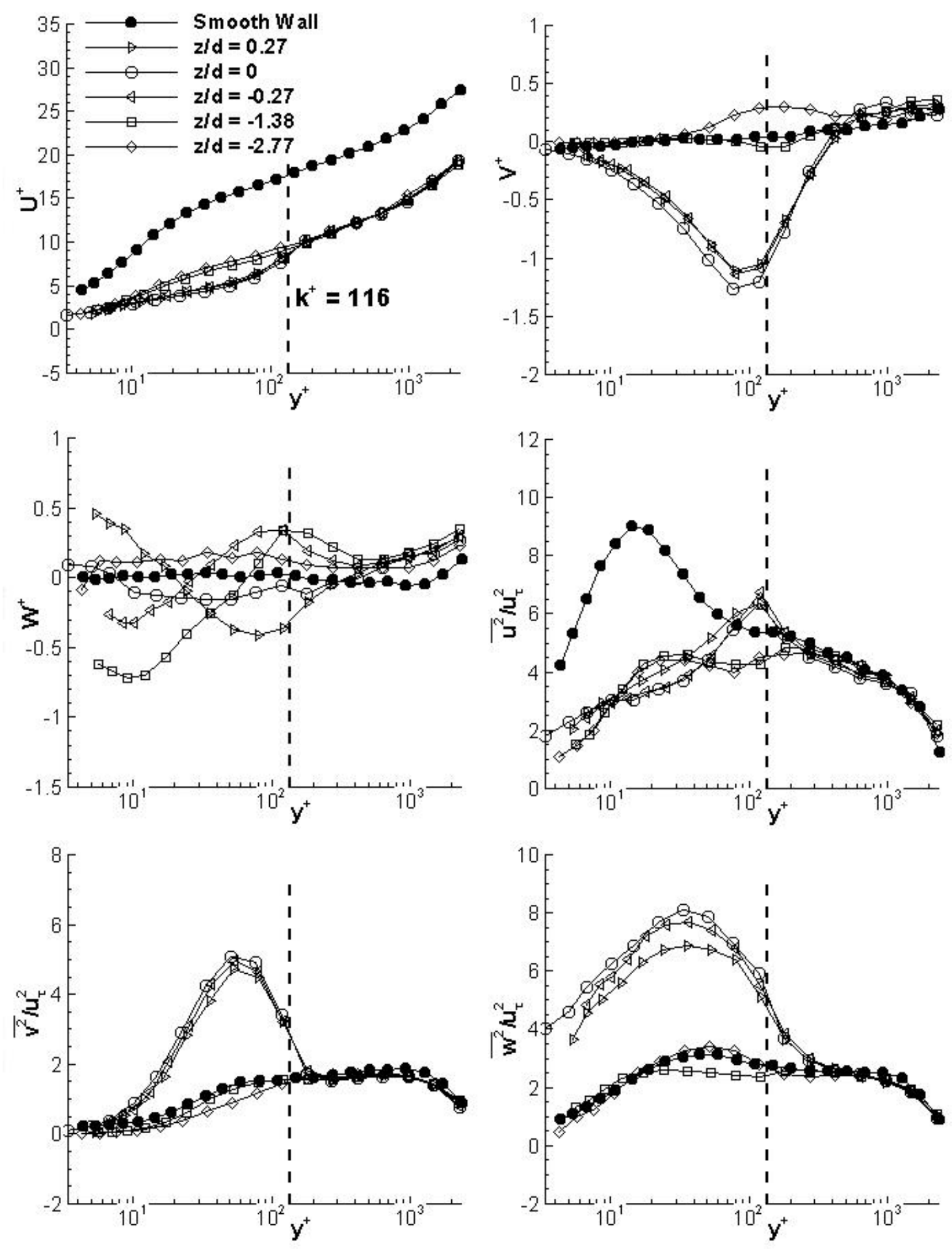

Figure A.53: $1.52 \mathrm{~mm}$ Cylinders, straight: Mean velocity and Reynolds normal stress mid-plane plots 

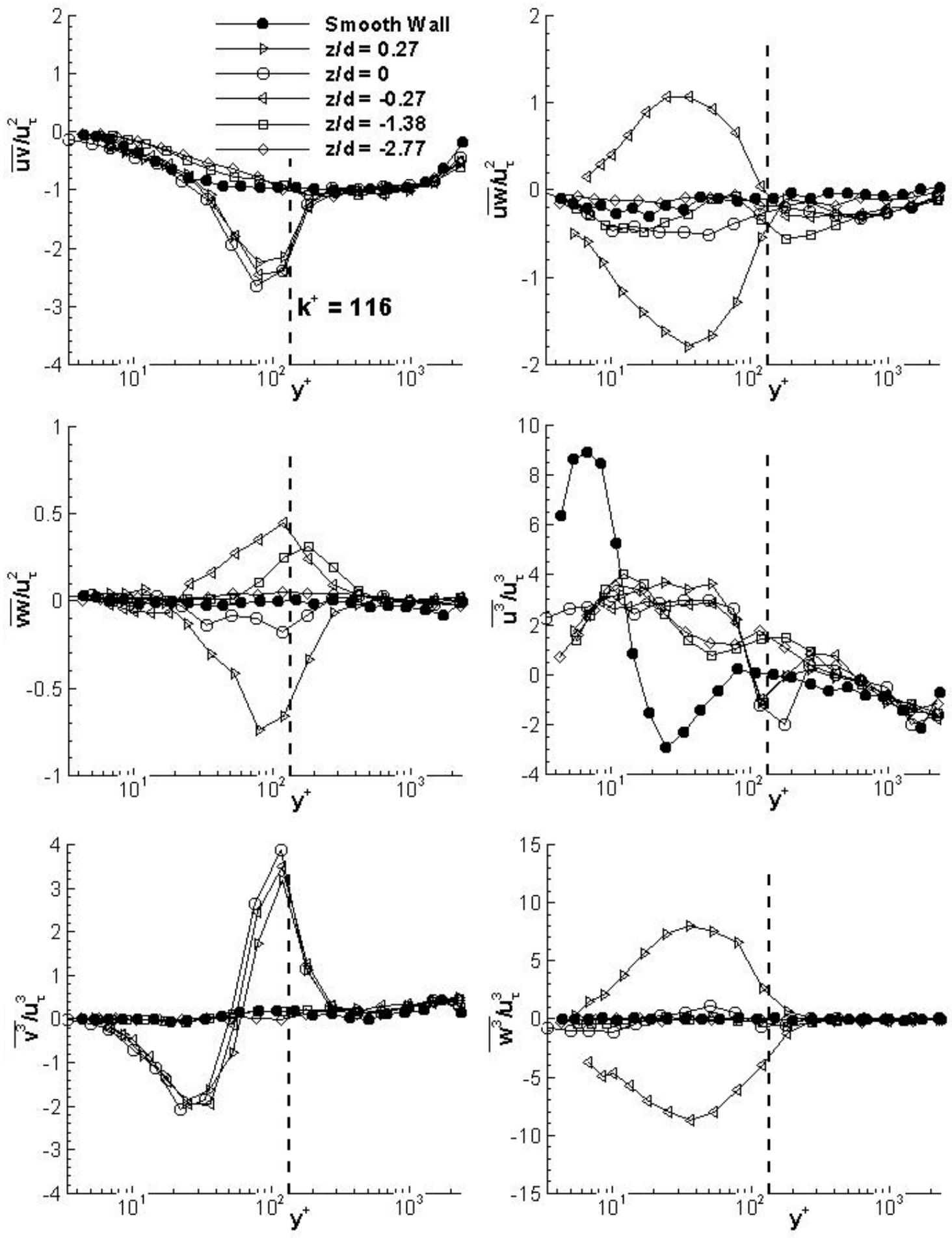

Figure A.54: $1.52 \mathrm{~mm}$ Cylinders, straight: Reynolds shear stress and triple product mid-plane plots 

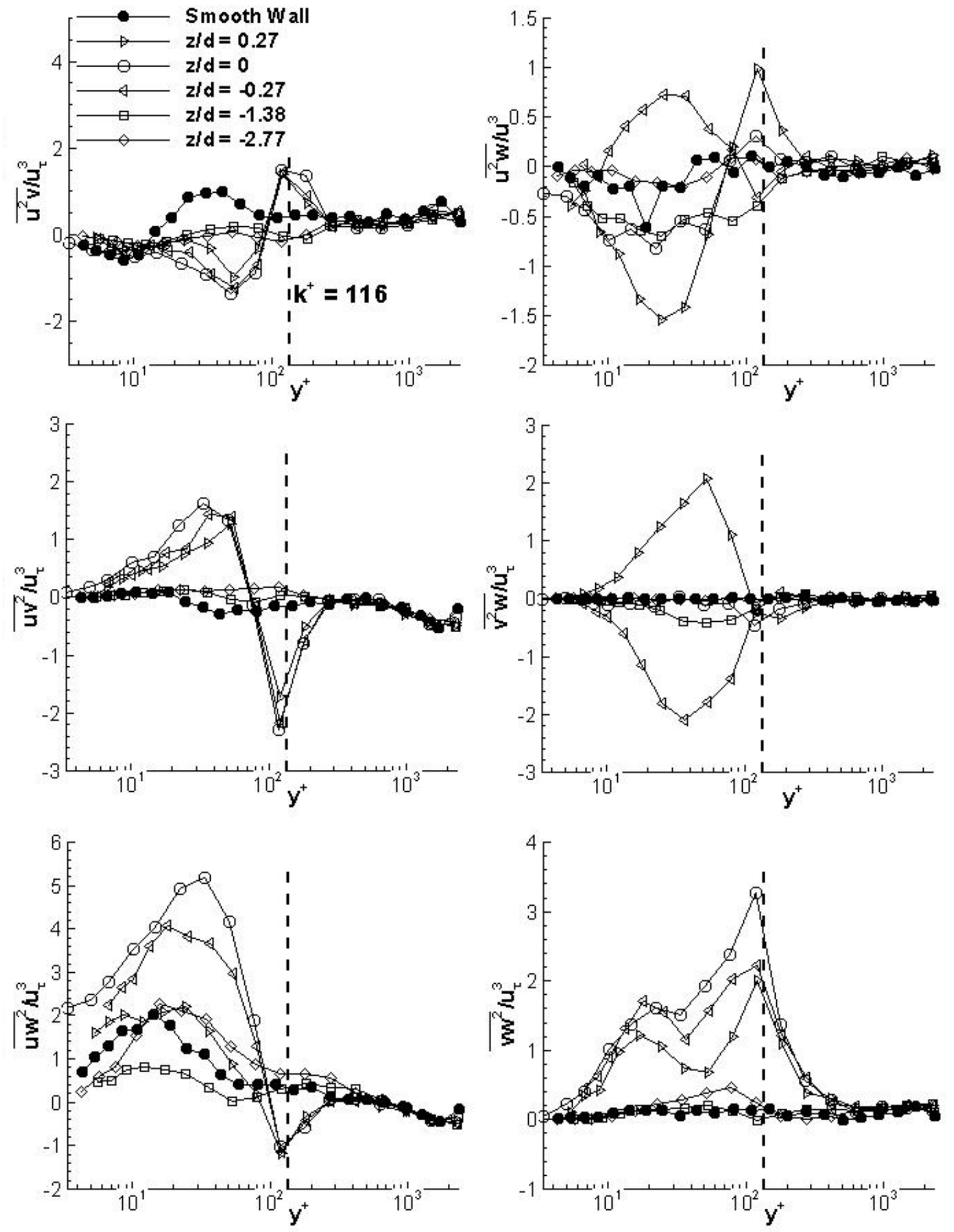

Figure A.55: $1.52 \mathrm{~mm}$ Cylinders, straight: Triple product mid-plane plots (continued) 

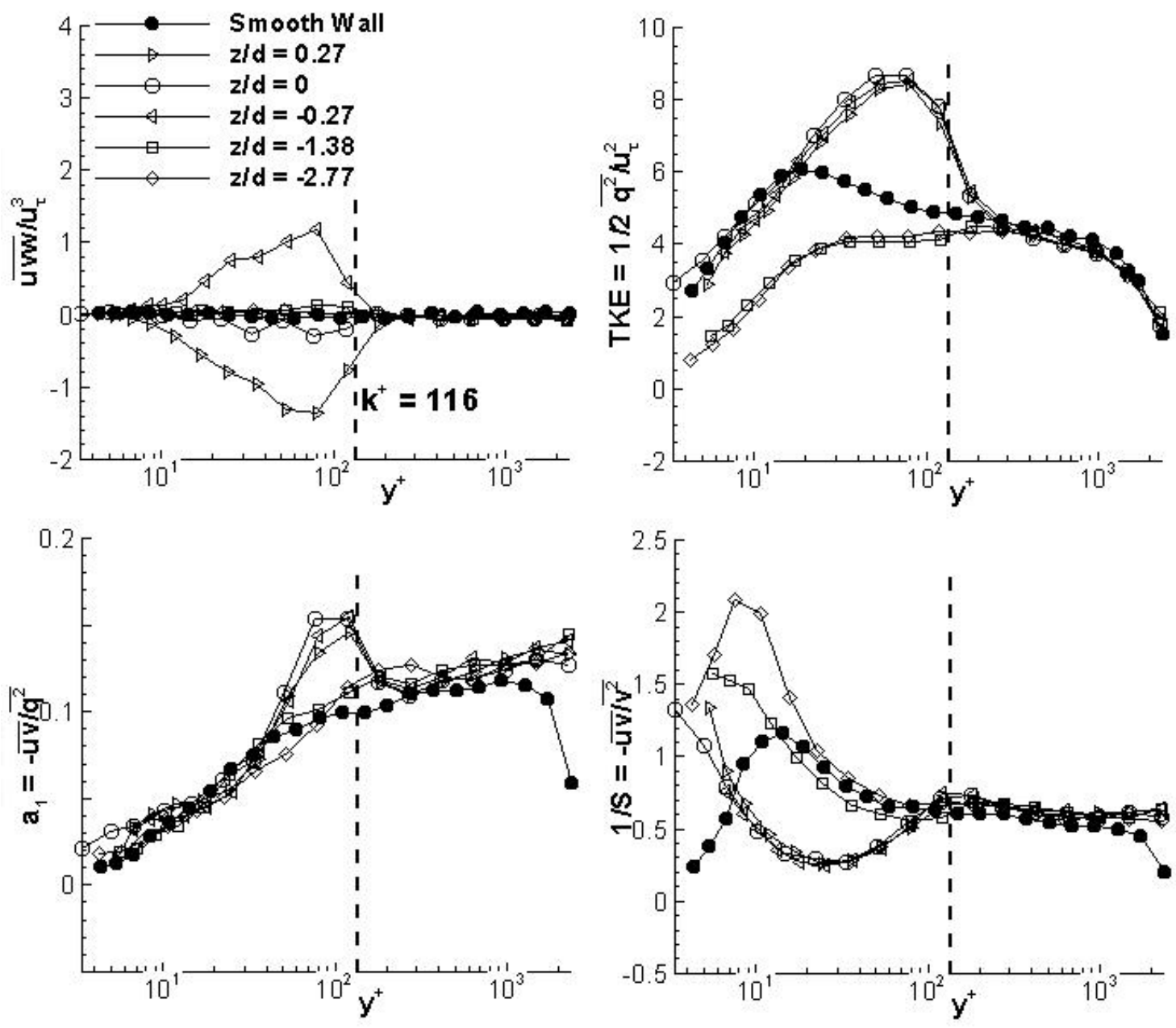

Figure A.56: $1.52 \mathrm{~mm}$ Cylinders, straight: $\overline{u v w} / u_{\tau}^{2}$ triple product, turbulent kinetic energy and structure parameters $a_{1}$ and $1 / S$ mid-plane plots 


\section{A.9 $\quad 1.52 \mathrm{~mm}$ Cylinders, Staggered}
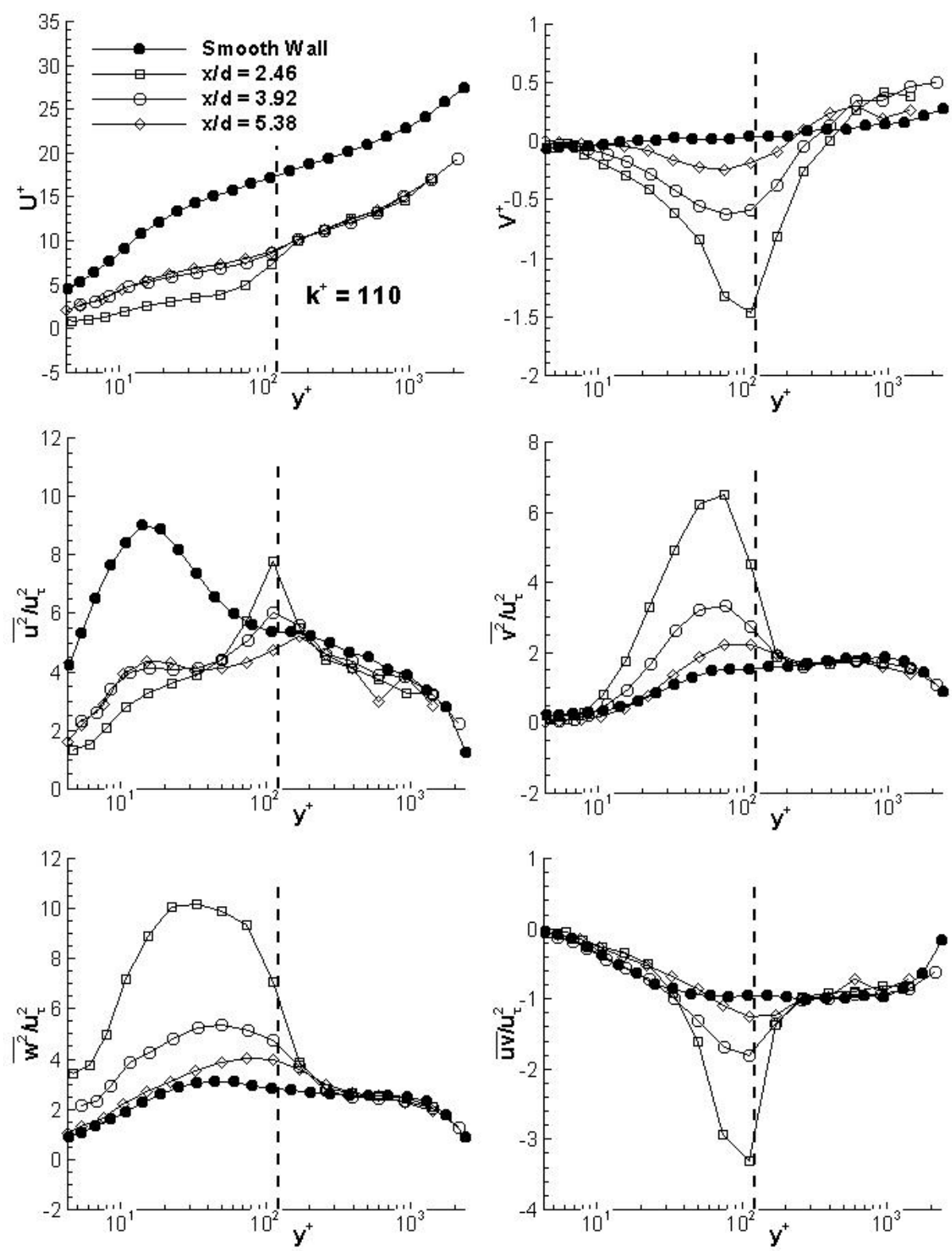

Figure A.57: $1.52 \mathrm{~mm}$ Cylinders, staggered: Mean velocity and Reynolds stress centerline plots 

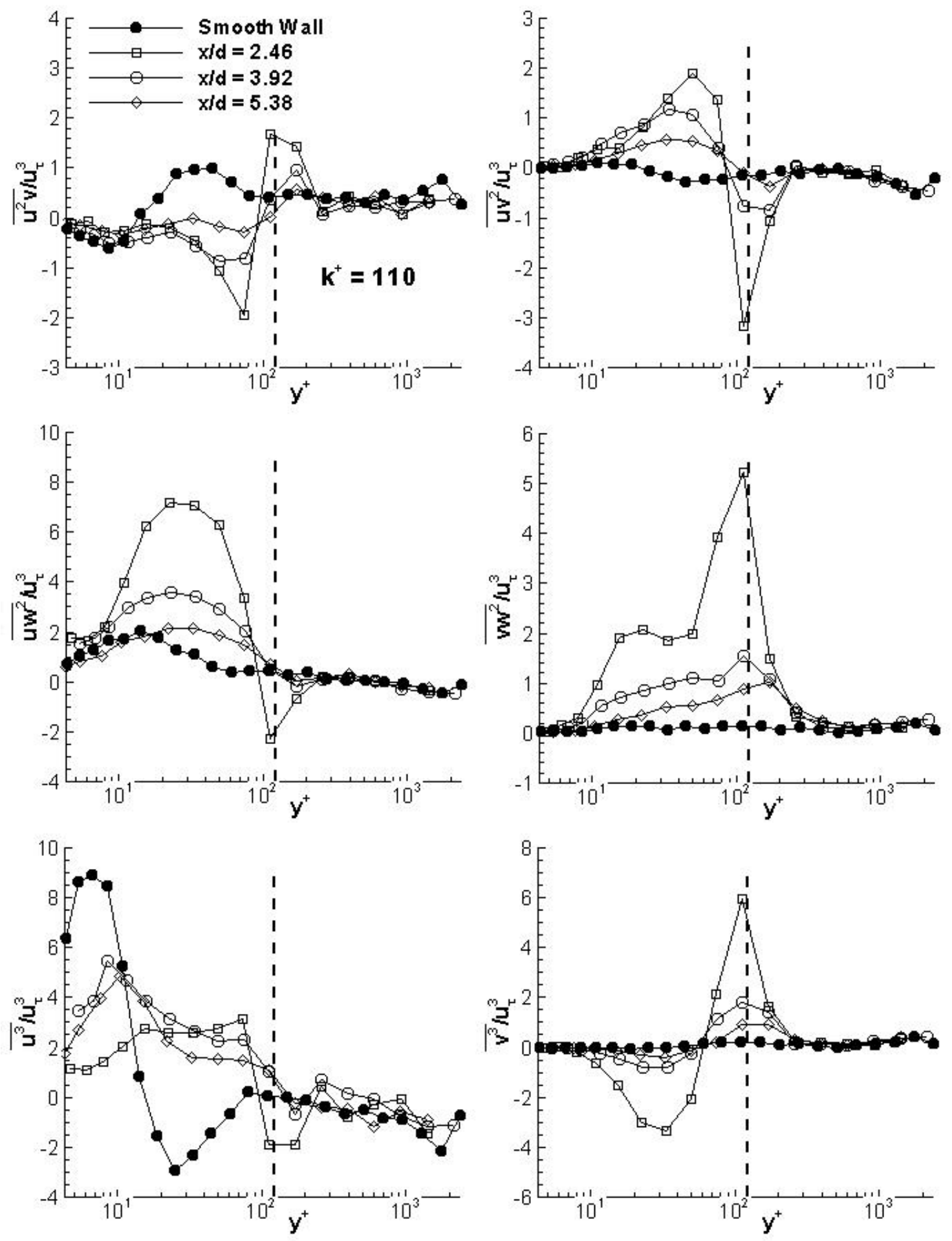

Figure A.58: $1.52 \mathrm{~mm}$ Cylinders, staggered: Triple product centerline plots 

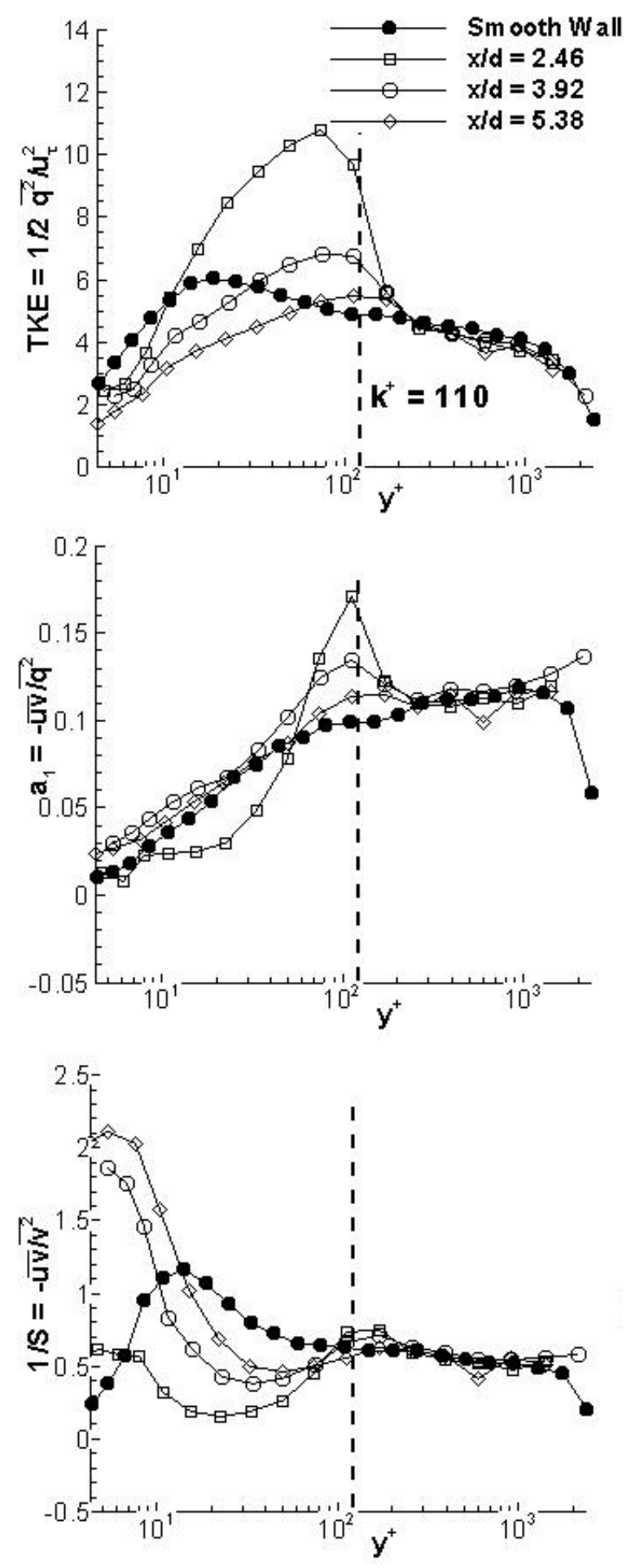

Figure A.59: $1.52 \mathrm{~mm}$ Cylinders, staggered: Turbulent kinetic energy and structure parameters $a_{1}$ and $1 / S$ centerline plots 

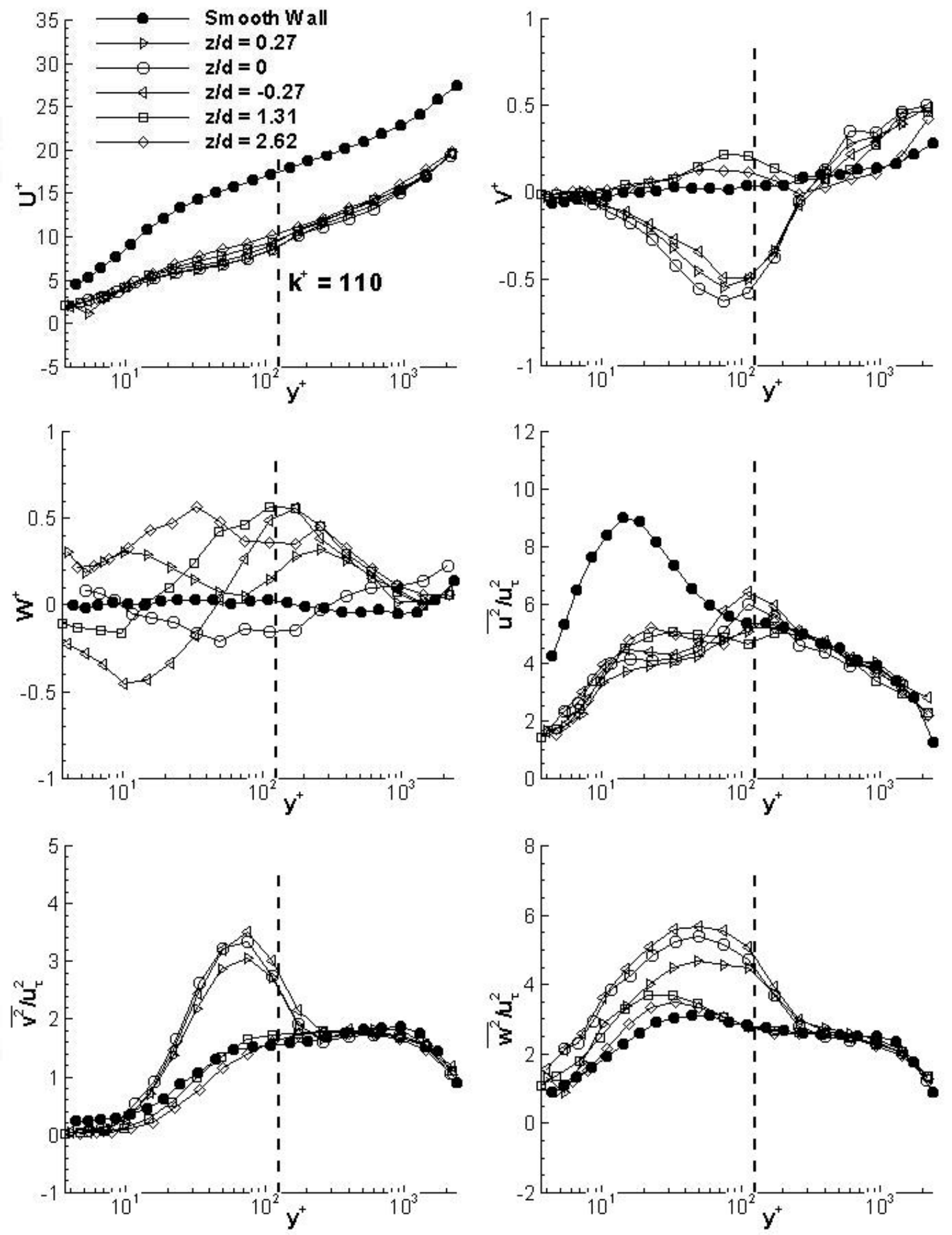

Figure A.60: $1.52 \mathrm{~mm}$ Cylinders, staggered: Mean velocity and Reynolds normal stress mid-plane plots 

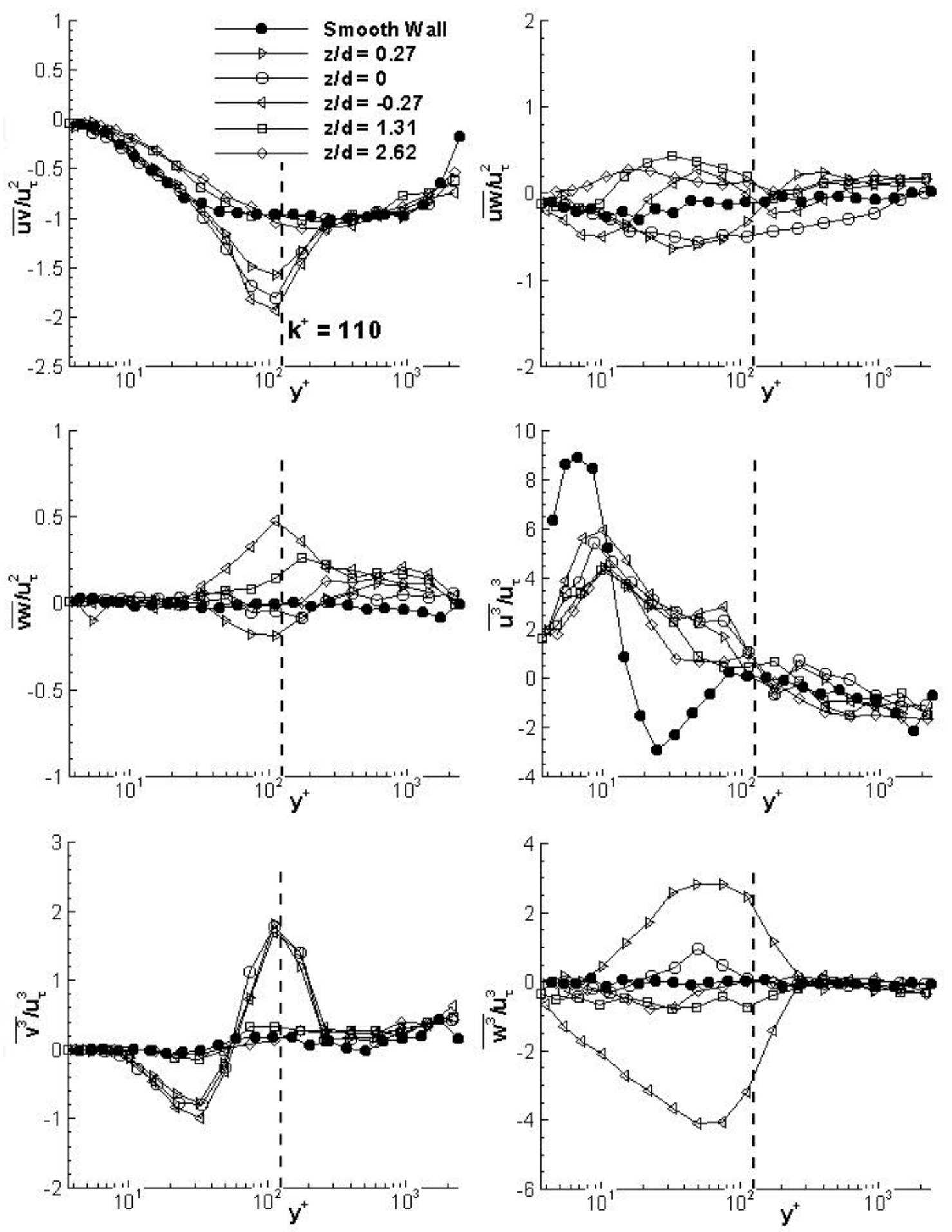

Figure A.61: $1.52 \mathrm{~mm}$ Cylinders, staggered: Reynolds shear stress and triple product mid-plane plots 

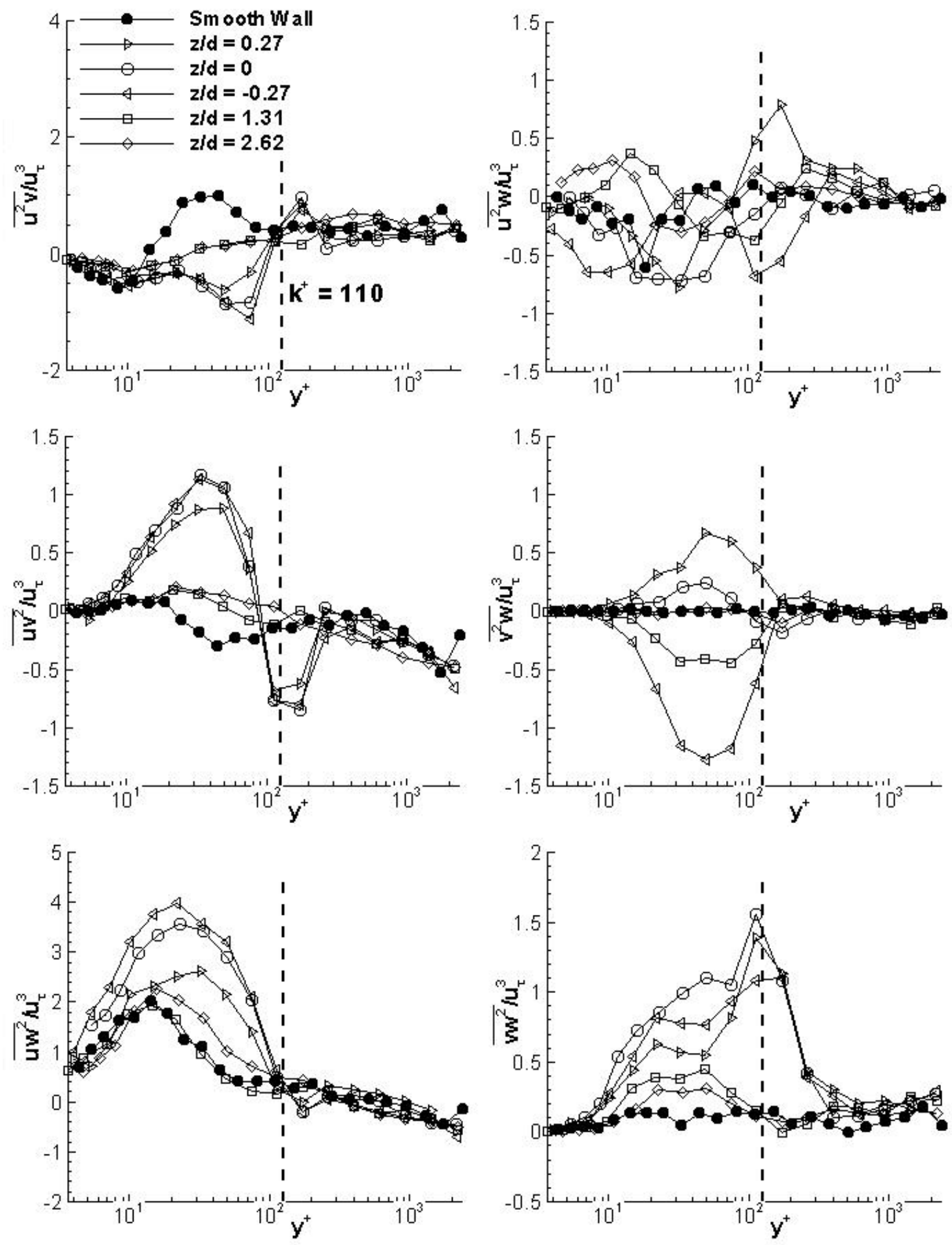

Figure A.62: $1.52 \mathrm{~mm}$ Cylinders, staggered: Triple product mid-plane plots (continued) 

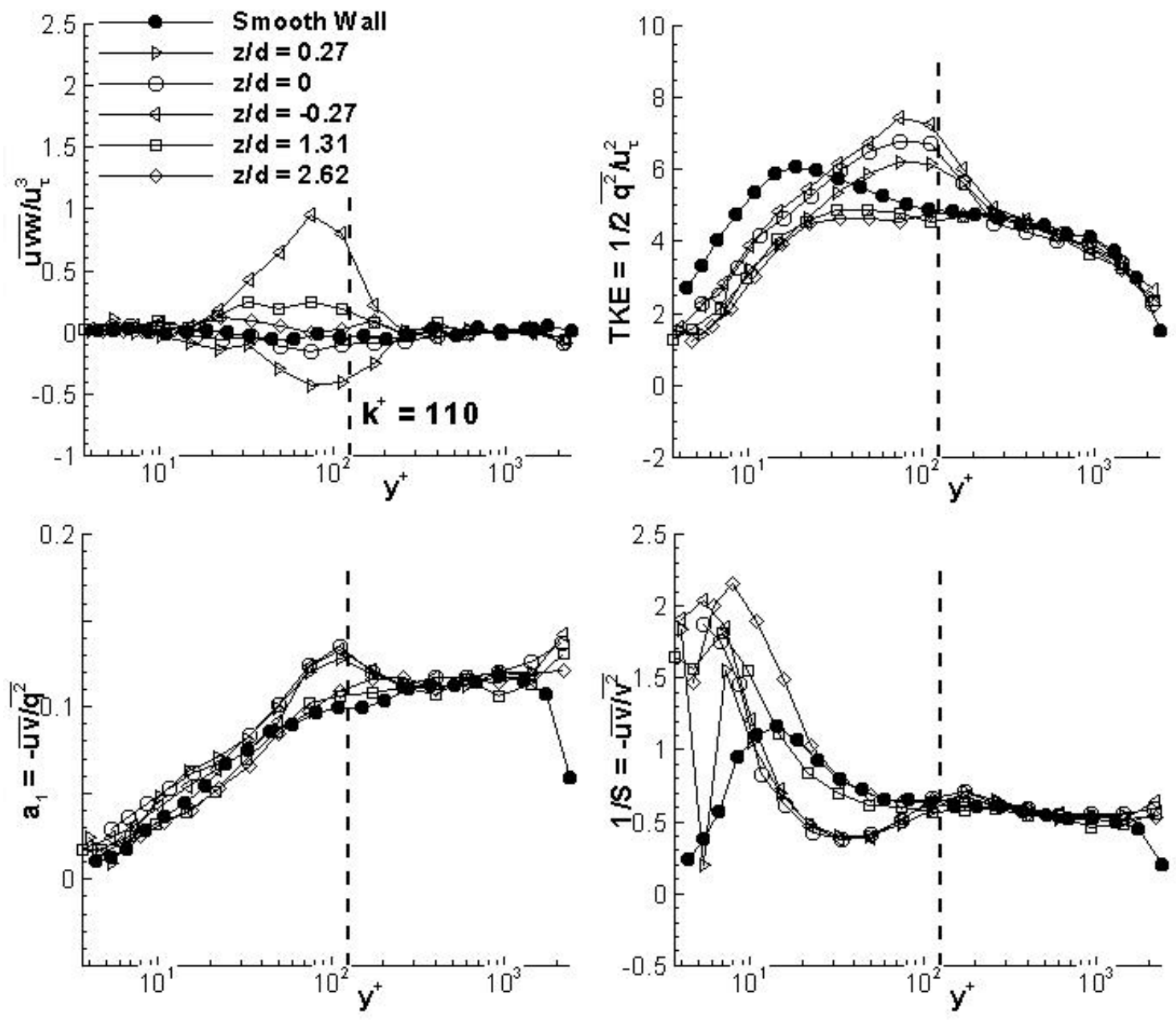

Figure A.63: $1.52 \mathrm{~mm}$ Cylinders, staggered: $\overline{u v w} / u_{\tau}^{2}$ triple product, turbulent kinetic energy and structure parameters $a_{1}$ and $1 / S$ mid-plane plots 


\section{Appendix B}

\section{Uncertainty Analysis}

\section{B.1 Wall Location}

The traverse upon which the LDV probe is mounted utilizes a linear encoder in the vertical direction, allowing for determination of position to within $1 \mu \mathrm{m}$. Thus, position can be set very accurately with respect to the wall location. However, there is some uncertainty in how this wall location is determined.

To find the wall, the laser probe is traversed until the measurement volume is on the glass wall. At the glass/air interface, the laser beams produce flare which is visible via a spectrum analyzer. The location of the wall is determined by traversing the probe through the glass surface several times and averaging the location at which the flare appears. By the nature of this method, uncertainty in wall position is the same magnitude as the measurement volume diameter, $\pm 50 \mu \mathrm{m}$

\section{B.2 Horizontal Measurement Location}

The method for determining the spanwise and streamwise location of the origin with respect to a roughness element was discussed in Section 2.5.3. The center of the roughness elements are found by repeatedly taking readings the location of the measurement volume on the element. By examining the variation in reading taken, the center of the roughness elements can be found within $\pm 60 \mu m$. 


\section{B.3 Free-stream velocity}

The free-stream velocity is set by a Pitot probe, and is not measured by the LDV system, so its uncertainty must be considered separately from that of the other velocity measurements. Using a liquid manometer, the dynamic pressure can be determined within $\delta h_{m}= \pm 0.02 i n H_{2} O$. The equation to determine velocity from the manometer measurement is,

$$
U_{\infty}=\sqrt{\frac{\rho_{H_{2} O}}{\rho_{a i r}} g h_{m}}
$$

Measurement uncertainty in temperature and pressure (used in determining the density of air) are small compared to manometer measurement error. Thus, root-mean-square (RMS) error is,

$$
\delta U_{\infty}= \pm \sqrt{\left(\frac{\partial U_{\infty}}{\partial h_{m}}\right)^{2}\left(\delta h_{m}\right)^{2}}
$$

Calculating derivatives of Equation B.1 and substituting, uncertainty in the free-stream velocity is determined to be $\delta U_{\infty}= \pm 0.1 \mathrm{~m} / \mathrm{s}$.

\section{B.4 Velocities}

The effect of various sources of uncertainty have been previously studied on the LDV system used in this study. Tang (2004) concluded that fringe bias, geometric bias, and angular bias are not significant in the LDV system used in this study because the measurement volume is small and nearly spherical. Kuhl (2001) showed that the lack of a correlation between velocity magnitude fluctuation and data rate fluctuation indicates that there is no velocity bias. Velocity gradient bias can be present in large velocity gradients, such as near a wall, but can be reduced by a small measurement volume. Tang (2004), using equations from Durst et al. (1992), showed that velocity gradient bias is less than the uncertainty in the data acquired by this system.

The uncertainties in the velocities, Reynolds stresses, and triple products are calculated by the same method described in Olçmen and Simpson (1995). 


\begin{tabular}{|c|c|c|c|}
\hline$U / U_{\tau}$ & \pm 0.27 & $\overline{u^{2} v} / U_{\tau}^{3}$ & \pm 0.06 \\
$V / U_{\tau}$ & \pm 0.01 & $\overline{u^{2} w} / U_{\tau}^{3}$ & \pm 0.10 \\
$W / U_{\tau}$ & \pm 0.10 & $\overline{v^{2} w} / U_{\tau}^{3}$ & \pm 0.01 \\
$\overline{u^{2}} / U_{\tau}^{2}$ & \pm 0.12 & $\overline{u v^{2}} / U_{\tau}^{3}$ & \pm 0.01 \\
$\overline{v^{2}} / U_{\tau}^{2}$ & \pm 0.05 & $\overline{u w^{2}} / U_{\tau}^{3}$ & \pm 0.03 \\
$\overline{w^{2}} / U_{\tau}^{2}$ & \pm 0.01 & $\overline{v w^{2}} / U_{\tau}^{3}$ & \pm 0.04 \\
$-\overline{u v} / U_{\tau}^{2}$ & \pm 0.03 & $\overline{u v w} / U_{\tau}^{3}$ & \pm 0.01 \\
$-\overline{u w} / U_{\tau}^{2}$ & \pm 0.08 & $\overline{u^{3}} / U_{\tau}^{3}$ & \pm 0.20 \\
$-\overline{v w} / U_{\tau}^{2}$ & \pm 0.03 & $\overline{v^{3}} / U_{\tau}^{3}$ & \pm 0.03 \\
& & $\overline{w^{3}} / U_{\tau}^{3}$ & \pm 0.06 \\
\hline
\end{tabular}

Table B.1: Velocity, Reynolds stress, and triple product uncertainties with $21: 1$ odds

Two smooth wall boundary layer profiles, acquired on different days, are utilized. Chauvenet's criterion is used to determine the standard deviation,

$$
\sigma=\frac{d_{\max }}{1.15}
$$

In this equation, $d_{\max }$ is the average of half of difference between data values for the two profiles. 21:1 odds uncertainties are equal to $\pm 2 \sigma$, and are shown in Table B.1.

Strictly speaking, these are calculations of repeatability. However, in this case, this does describe the uncertainty as well. Comparison with smooth wall profiles, and analysis appearing in Kuhl (2001) and Tang (2004), confirm that biases have been calibrated out of the system. Thus, scatter in the data is a result of the accumulated uncertainties in the system. As discussed in Olçmen and Simpson (1995), comparison of velocity profiles, and application of Chauvenet's criterion, yields the uncertainty for the measured quantities.

The Outer Layer LDV system has a larger measurement volume, which increases uncertainties, and the one-component configuration introduces additional error, such as velocity bias. Thus, the uncertainty in the velocity measured by the Outer Layer LDV system is $\delta U_{\infty}= \pm 0.1 \mathrm{~m} / \mathrm{s}$. 


\begin{tabular}{|c|c|}
\hline Configuration & $\delta u_{\tau}$ \\
\hline \hline Gaussian, 5.49 mm spacing, straight & 0.022 \\
\hline Gaussian, 5.49 mm spacing, staggered & 0.068 \\
\hline Gaussian, 8.23 mm spacing, straight & 0.042 \\
\hline Gaussian, 8.23 mm spacing, staggered & 0.090 \\
\hline $0.38 \mathrm{~mm}$ cylinder, straight & 0.022 \\
\hline $0.38 \mathrm{~mm}$ cylinder, staggered & 0.026 \\
\hline $0.76 \mathrm{~mm}$ cylinder, straight & 0.027 \\
\hline $0.76 \mathrm{~mm}$ cylinder, staggered & 0.030 \\
\hline $1.52 \mathrm{~mm}$ cylinder, straight & 0.038 \\
\hline $1.52 \mathrm{~mm}$ cylinder, staggered & 0.038 \\
\hline
\end{tabular}

Table B.2: Skin friction velocity uncertainties with 20:1 odds

\section{B.5 Skin Friction Velocity}

The uncertainty in the skin friction velocity, $u_{\tau}$, was calculated for 20:1 odds (95\% confidence interval) for each roughness case,

$$
\delta u_{\tau}=\frac{1.96 \sigma}{\sqrt{n}}
$$

$n$ is the number of log-layer data points used in the calculation of $u_{\tau}$. Calculated uncertainties for skin friction velocities appear in Table B.2. Note that the largest uncertainties are associated with the larger Gaussian elements. This is a result of the fact that not only is the standard deviation of the data larger than that of the cylindrical data, but the smaller log layer (due to the larger $k / \delta$ ) results in a fewer points, $n$, used in the calculation of $u_{\tau}$.

\section{B.6 Turbulent Kinetic Energy}

Recall that non-dimensional TKE is equal to half the sum of the normal stresses, $\left(\overline{u^{2}}+\overline{v^{2}}+\overline{w^{2}}\right) / 2$. The root-mean-square error is calculated by,

$$
\delta T K E= \pm \sqrt{\left(\frac{\partial T K E}{\partial \overline{u^{2}}}\right)\left(\delta \overline{u^{2}}\right)^{2}+\left(\frac{\partial T K E}{\partial \overline{v^{2}}}\right)\left(\delta \overline{v^{2}}\right)^{2}+\left(\frac{\partial T K E}{\partial \overline{w^{2}}}\right)\left(\delta \overline{w^{2}}\right)^{2}}
$$


After taking the partial derivatives, Equation B.5 is evaluated using uncertainties for the normal stresses from Table B.1. The uncertainty in turbulent kinetic energy is calculated to be $\delta T K E= \pm 0.065 \mathrm{~m}^{2} / \mathrm{s}^{2}$. 


\section{Appendix $\mathrm{C}$}

\section{Monodisperse Seeding Study}

\section{C.1 Introduction}

Laser Doppler Velocimetry (LDV) is a method of taking instantaneous velocity measurements in fluid flows (e.g. air or water) with very precise spatial resolution. These measurements are accomplished by focusing multiple laser beams into a small $(\approx 50 \mu m)$ measurement volume within the fluid flow. Small seed particles injected into the flow pass through this measurement volume, and reflect some of the laser light. This reflected light is then received, and the Doppler shift of the light's frequency can be related to the velocity of the seed particle, which is assumed to be equal to the air velocity.

The following study is related to research done in the Virginia Tech Small Boundary Layer Wind Tunnel, a small (10 cm x $23 \mathrm{~cm}$ cross section), closed loop wind tunnel. The study's purpose is to investigate various methods of seeding the wind tunnel and make a recommendation on the most effective method.

For the purpose of LDV measurements, it is important to have monodisperse (consistently-sized) particles. Small particles reflect too little light, and appear in the received signal only as noise, and particles which are too large fail to follow the flow correctly, and the resulting measurements therefore fail to correctly represent the flow conditions.

Two different types of seed material were investigated here: Polystyrene Latex (PSL) spheres and di-octyl phthalate (DOP). PSL spheres, shown in 
Figure C.1 are small $(\approx 1 \mu \mathrm{m})$ solid particles with typical standard deviations being around 3.5\% of the particle diameter (Alfrey et al., 1954). This narrow size distribution makes them an ideal seeding material. The PSL spheres come suspended in water, so the challenge with this seed material is to atomize a sufficient amount of the sphere and water solution to obtain an acceptable data rate, and also to have the atomized droplets dry fast enough so that only the solid sphere remains when the particle passes through the LDV system's measurement volume. Various methods of atomizing and injecting PSLs into the wind tunnel are investigated in this study.

DOP is a liquid that is atomized by a nozzle to form small droplets; these droplets are the seed material for the LDV measurements. Previous to this study, the seeding system employed was a Laskin nozzle aerosol generator that atomized DOP with a high pressure jet of air. Though this system produced a large volume of seed, the particles produced had a very large size distribution, as documented in a previous analysis performed at the University of Arkansas Graduate Institute of Technology (Mazumder, 1981). Figure C.2 shows a typical particle size distribution produced by a Laskin nozzle generator. In this study, an improvement to this system is investigated.

\section{C.2 Seeder Output Requirements}

To determine the seed density requirement, it was assumed that four particles need to arrive in the measurement volume within $30 \mu s$ to yield accurate instantaneous gradients. In order to calculate the concentration, a representative mean streamwise velocity in the boundary layer must be chosen. At velocities lower than this, fewer 4 particle events will occur, while at higher velocities, 4 particle events may occur within a shorter time window. In this case, a velocity of $10 \mathrm{~m} / \mathrm{s}$ is chosen, corresponding to a y-location of approximately $245 \mu \mathrm{m}$. For a measurement volume diameter of $150 \mathrm{~m}$, the volume of air passing through the measurement volume in $30 \mu \mathrm{s}$ is,

$$
V_{\text {air }}=A_{M V} U_{\text {rep }} t_{\text {tpart }}
$$

where $V_{\text {air }}$ is the volume of air, $A_{M V}$ is the projected area of the measurement volume, $U_{\text {rep }}$ is the chosen mean streamwise velocity, and $t_{4 p a r t}$ is the time window for 4 particles. In this volume of air, then, there must be 4 


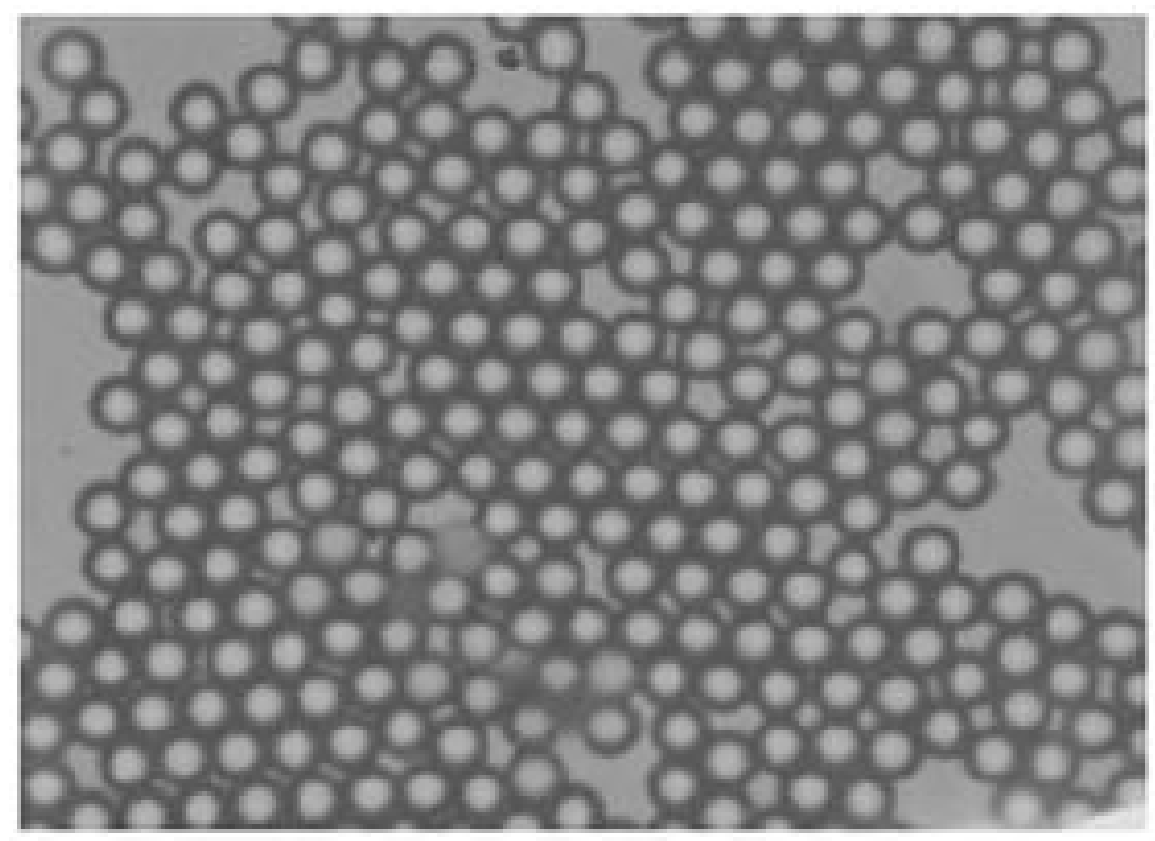

Figure C.1: $2.7 \mu m$ PSL spheres magnified 2000x. Ref. Meyers (1991).

seed particles, allowing for the calculation of overall seed density in the test section.

$$
C=\frac{4 \text { particles }}{V_{\text {air }}}=7.55 \mathrm{e} 11 \text { particle } / \mathrm{m}^{3}
$$

To determine the output required of the seeder, the flow in the tunnel boundary layer must be characterized. Boundary layer volumetric flow rate is

$$
Q_{B L}=U_{\infty}\left(\delta-\delta^{*}\right) W
$$

where $Q_{B L}$ is the boundary layer volumetric flow rate, $U_{\infty}$ is the free stream velocity, $\delta$ is the boundary layer thickness, $\delta^{*}$ is the displacement thickness, and $W$ is the width of the tunnel. The parameters have the approximate values as follows:

$$
\begin{array}{r}
U_{\infty}=27.5 \mathrm{~m} / \mathrm{s} \\
\delta=40 \mathrm{~mm} \\
\delta^{*}=6.3 \mathrm{~mm} \\
W=229 \mathrm{~mm}
\end{array}
$$




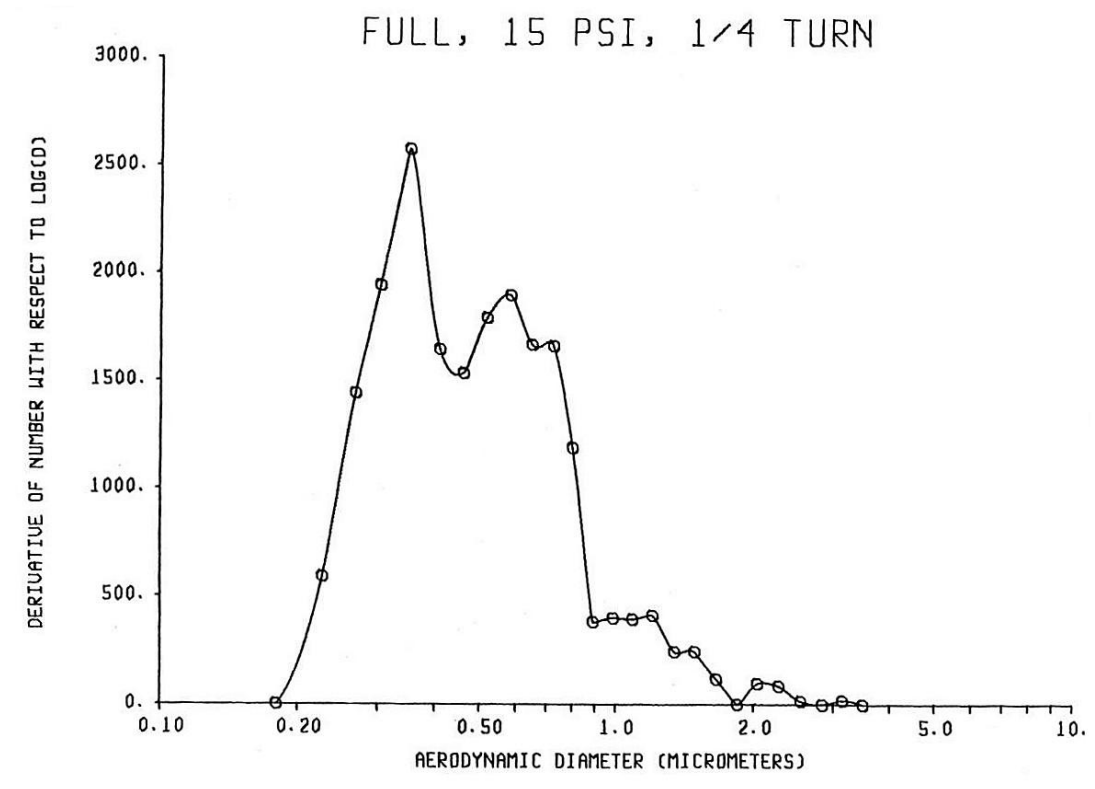

Figure C.2: Representative histogram of particle size distribution produced by a Laskin nozzle seeder. Ref. Mazumder (1981). 
Therefore, the boundary layer volumetric flow rate is about $Q=0.2119 \mathrm{~m}^{3} / \mathrm{s}=$ $12.7 \mathrm{~m}^{3} / \mathrm{min}$. Thus the seeder output must be,

$$
r=C \cdot Q=9.58 e 12 \text { particle } / \text { min }
$$

This provides an upper limit for what needs to be produced by the seeding apparatus, since it would produce many more 4 particle events than necessary, especially away from the wall, and that calculation of the seeder output in Equation C.5 assumes that there is no recirculation of seed material.

\section{C.3 Polystrene Latex (PSL) Sphere Seeding}

Because of their extremely consistent size, PSL spheres are an attractive option for seeding a wind tunnel. In this section, a variety of seeding methods that were investigated are described and evaluated.

\section{C.3.1 Injection of Seed into Tunnel}

The PSL spheres are injected into the second contraction, about $10 \mathrm{~cm}$ downstream of the turbulence screens. This location was chosen because the flow velocity is relatively low, so disturbance to the structure of the boundary layer in the test section would be negligible. In addition, wall curvature at this point is small, facilitating the attachment of the injection apparatus.

The complete injection apparatus can be seen in Figure C.3. The PSLs exit the seeder box through six $1 \mathrm{~cm}$ inner diameter rubber tubes. These six tubes exhaust through six holes that are evenly-spaced spanwise across the contraction floor. The tubes are attached to the contraction via $2.5 \mathrm{~cm}$ lengths of copper tubing that are held in place by a flange attached to the outside of the tunnel.

To eliminate the effect of having a jet at the injection point, a baffle plate (Figure C.4) is employed. The baffle plate is mounted inside the tunnel, so that the oncoming flow sees only a ramp followed by a slight backstep. 


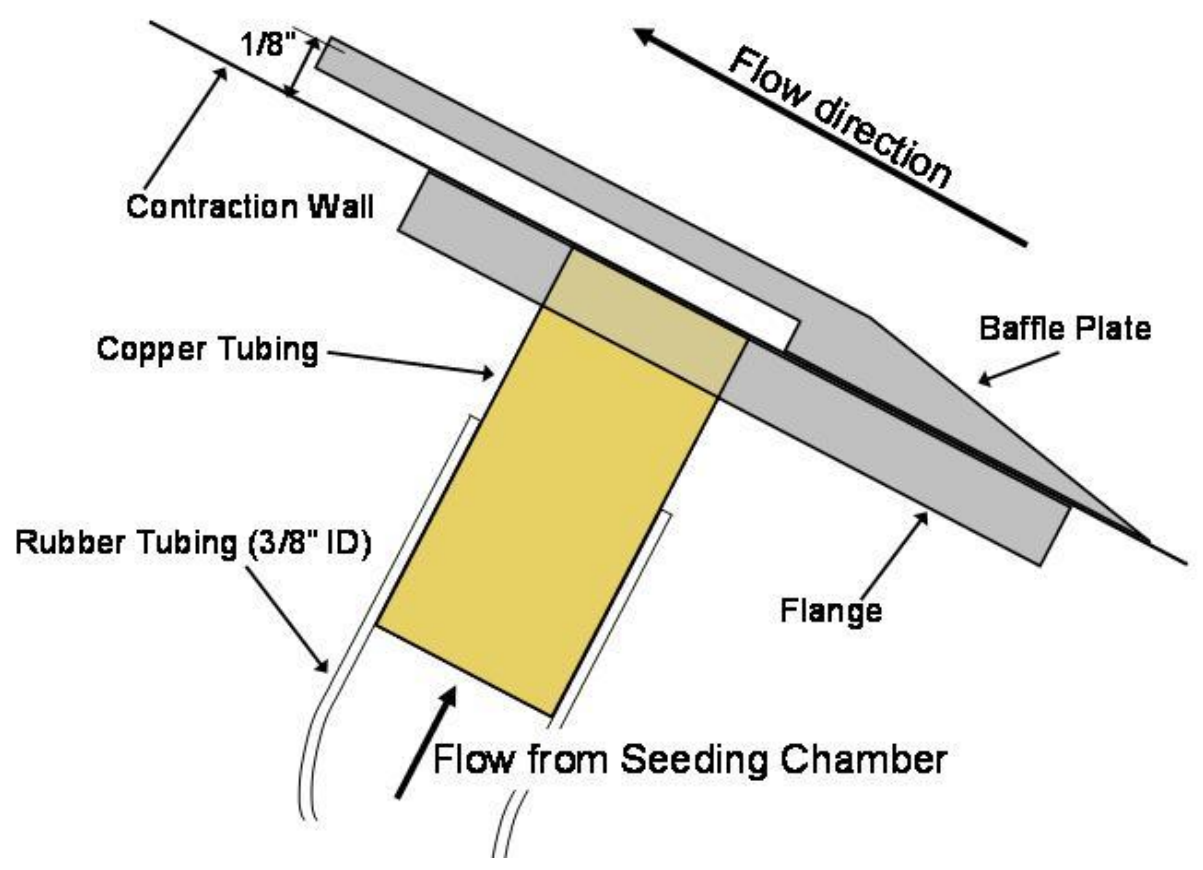

Figure C.3: Seed injection apparatus (not to scale)

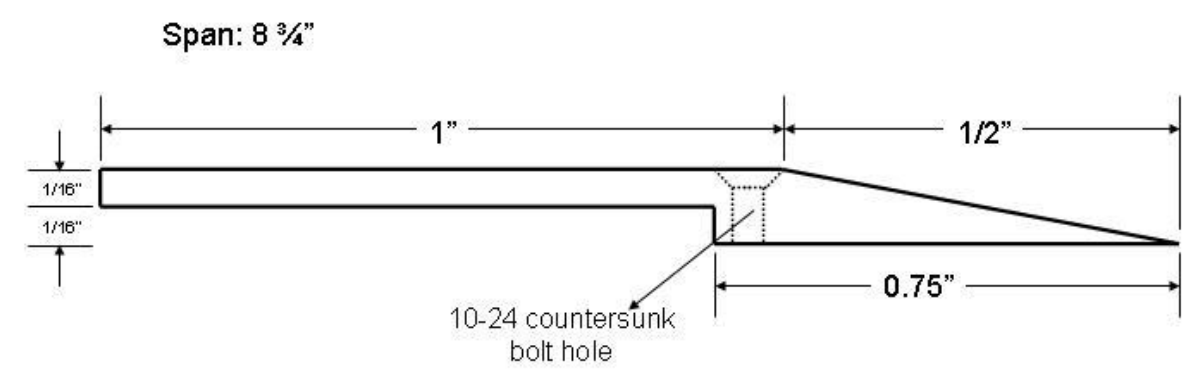

Figure C.4: Baffle plate (side view) 


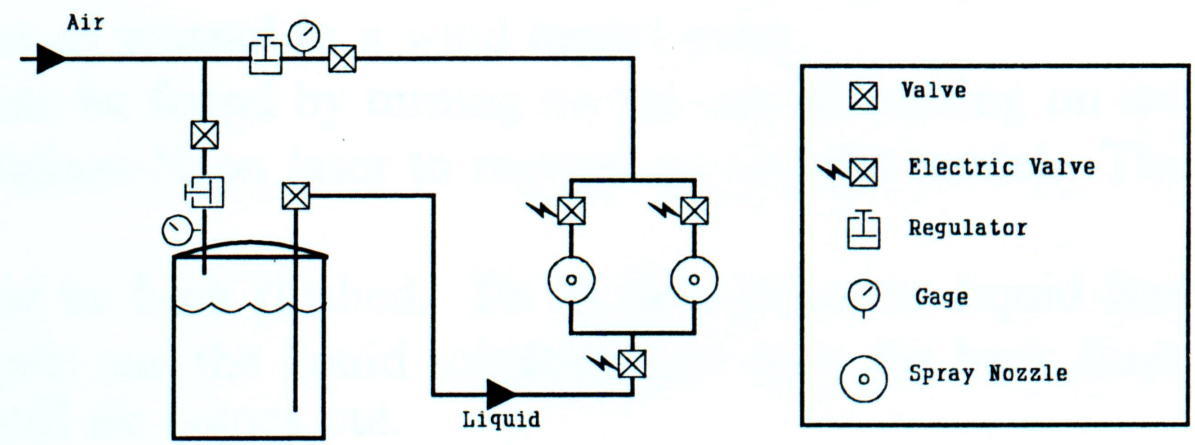

Figure C.5: Schematic of Spray Systems nozzle apparatus. Ref. Chesnakas (1995).

The injected aerosol impacts on the underside of the baffle plate, serving to evenly distribute the seed across the width of the tunnel. The seed is then entrained in the flow coming over the backstep. Spanwise bars and sand grain roughness at the exit of the contraction (see wind tunnel description) eliminate any disturbance created by the flow over the baffle plate.

Note that the injection point and baffle plate are located only five feet ahead of the measurement location in the test section. Thus, seed must be very dry when produced, or dried outside the wind tunnel, because after injection there is minimal drying time.

\section{C.3.2 Spray Systems Nozzles}

Previous work was done by Chesnakas (1995) using PSL spheres as the seed material in the Virginia Tech Stability Wind Tunnel. The seeding system employs two Spray Systems Inc. air atomizing nozzles to produce a fine mist of PSL solution. Figure C.5 is a schematic of the system employed. A compressed air line is split, so that one line goes to pressurize the tank containing the seed solution, and the other line goes to the nozzles. Pressurized liquid leaves the tank through another hose. The air and liquid feed lines go to the nozzle assembly (Figure C.6), which consists of the two nozzles, and three valves to turn air and liquid flows on and off.

One of the keys to successful PSL seeding is correct droplet size. The 


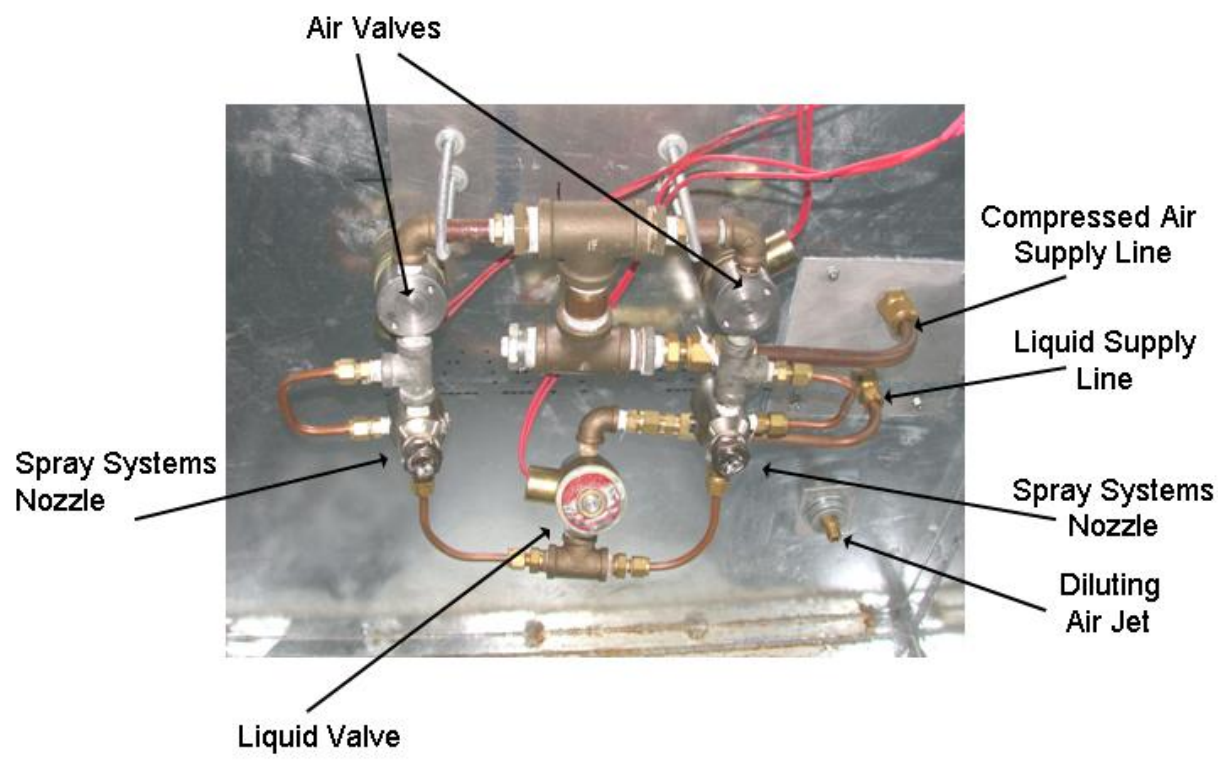

Figure C.6: Spray Systems nozzle assembly, mounted inside large plenum 
droplet must be large enough to contain one PSL sphere, but not so large that it contains multiple particles or that the liquid doesn't evaporate quickly. In the case of this system, droplet size is controlled by the ratio of the pressures in the liquid and air lines. The greater the air pressure relative to the liquid pressure, the dryer the aerosol, and, conversely, the closer the pressures get to each other, the droplet size is larger and the aerosol becomes very wet.

The tests done were conducted by placing the nozzle assembly into a small settling chamber, with the nozzle pointed upwards. This plenum measures $51 \mathrm{~cm}$ in height, $76 \mathrm{~cm}$ in length and $41 \mathrm{~cm}$ in width. Aerosol would then exit through the six tubes in the top of the chamber and into the wind tunnel as discussed in the previous section. A connection was installed in the side of the settling chamber for a second compressed air line, providing diluting air to the chamber. This served to increase the settling chamber's static pressure and increase the flowrate into the tunnel.

Previously, internal mix nozzles were employed on the nozzle apparatus. Due to the nature of these nozzles, the maximum pressure difference that could be maintained between the air and liquid lines was approximately 10 psi. This difference was not sufficient, and the vast majority of the seed material impacted on the top and sides, and proceeded to pool in the bottom of the settling chamber.

The air cap was replaced to put the nozzles into an external mix configuration. Liquid and air pressures are able to be adjusted independently with external mix nozzles, allowing more freedom to create a larger pressure difference. Though this resulted in an improvement in the aerosol quality, the majority of the seed droplets still impacted in the settling chamber. A second, larger settling chamber (Figure C.7) was designed in order to provide a larger drying distance before the PSLs enter the wind tunnel. In this larger settling chamber, the nozzles were oriented parallel to the ground, so the seed aerosol travels down what is essentially a $1.83 \mathrm{~m}$ duct before making a 90 degree turn into the tubes that enter the tunnel. A co-flowing air line injected air in the same direction as the spray nozzles.

Though the air and liquid lines were adjusted through a wide range of pressures, little success was had with this arrangement. Though a fine, dense aerosol could be produced, visual inspection revealed that the droplets were purely liquid that quickly evaporated, and contained no PSLs to leave behind. Pressure ratios that produced a wetter aerosol consisted mainly of larger 

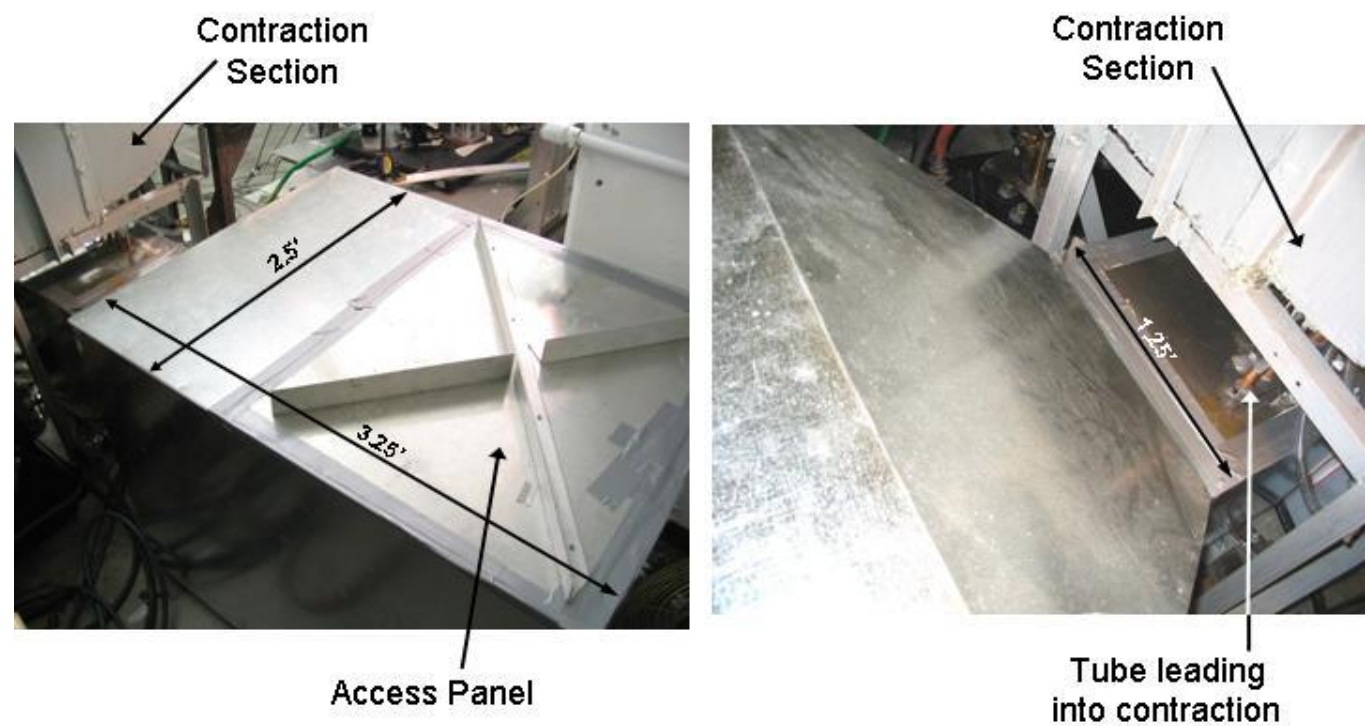

Figure C.7: Large plenum box

droplets that impacted on the walls and ceiling of the chamber, or were too heavy to travel the length of the duct and sank to the floor. These effects were evidenced by the large amounts of PSL material deposited on the inside of the settling chamber, despite the fact that very little seed material was leaving the tubes at the end of the chamber to enter the tunnel.

In this, and other systems to be discussed, the diluting liquid for the PSL solution was either ethanol or a 50/50 ethanol and water mix. Chang and Okuyama (2002) suggested that this second mixture is a faster-evaporating solution, but no significant improvement was noticed with this nozzle assembly. In another attempt to facilitate evaporation, heating tapes were wrapped around the liquid and co-flowing air lines before they entered the settling chamber. This produced no noticeable effect.

\section{C.3.3 Six-Jet Atomizer}

In an attempt to produce a dry PSL aerosol, a TSI, Inc. Model 9306 SixJet Atomizer was tested. The atomizer is a relatively simple and convenient instrument to use. The unit is a container that serves both as a reservoir for the liquid and encloses the six jet apparatus. There is an outlet tube on the 


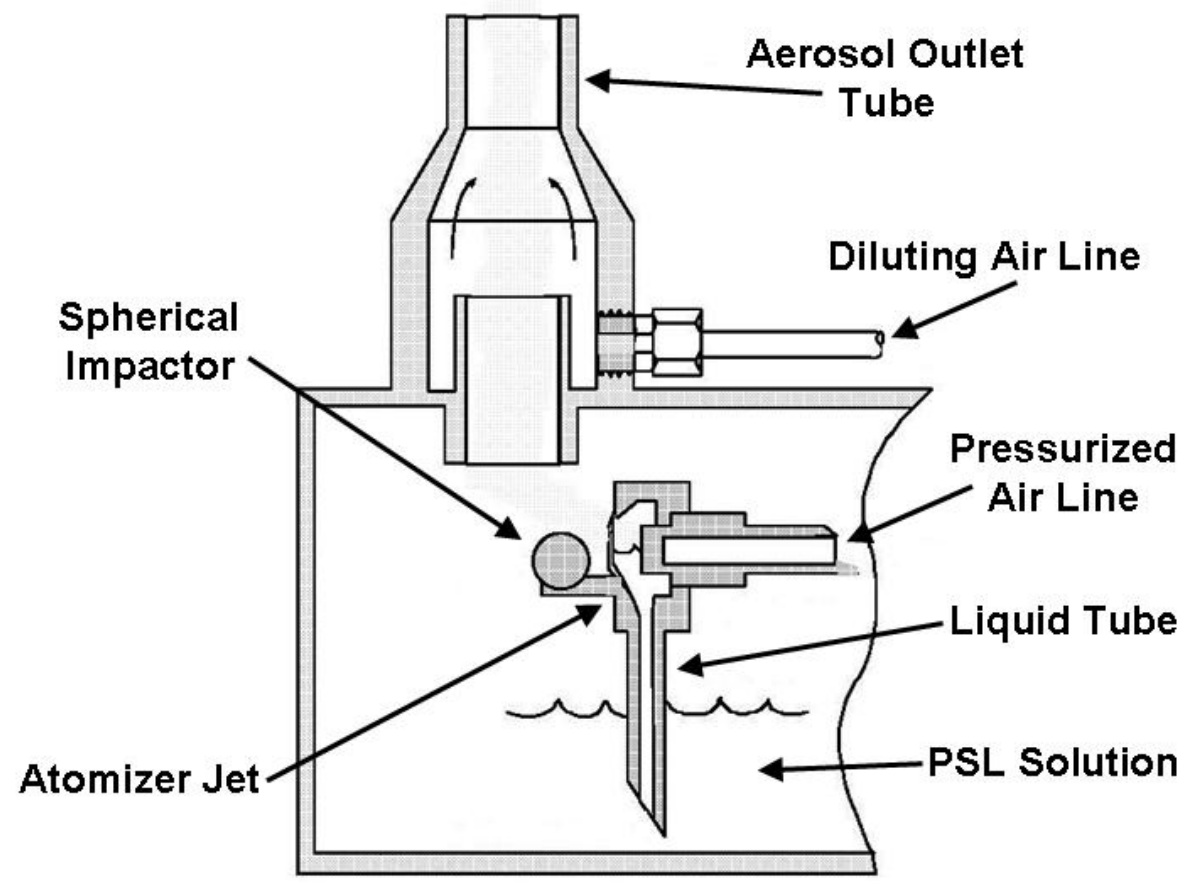

Figure C.8: TSI Six-Jet Atomizer drawing. Ref. TSI (2003).

top for the seed aerosol. Figure C.8 is a schematic of the unit and one of its jets. Compressed air creates a jet as it passes through a $0.381 \mathrm{~mm}$ orifice. This jet draws liquid up the tube which extends into the liquid reservoir below the jet assembly. At the top of this tube, the liquid is sheared off into droplets which exit the nozzle. A spherical impactor placed $0.254 \mathrm{~mm}$ from the exit of the nozzle serves to block any large droplets, leaving only an aerosol consisting of smaller, quick-drying particles. The aerosol exits through a outlet on the top of the unit.

Various adjustments can be made to control the nature of the seed aerosol output. Pressurized air can be varied between approximately 20 and 50 psi. Over this range, the most dense seed was produced at lower pressures, between 25 and 30 psi. Co-flowing air can be introduced in the outlet to dilute the aerosol and increase the flowrate. This was not used since flowrate out of the atomizer was not important; flowrate out of the seeding chamber in which the atomizer was to be contained was of more importance, and this could be controlled with the diluting air already installed on these chambers. 
The final variable was the mixture of the seed solution. Tests were first run with a mixture with the same particle loading used by Chesnakas (1995), but this solution was found to be much too dilute. Various other mixtures were tried, ranging all the way to undiluted PSL solution. Both undiluted PSL solution and a 50/50 mix of PSL solution and ethanol produced seed clouds of acceptable density.

With these high particle loadings, one problem encountered was that the nozzles tended to clog. More than likely, this was caused by a buildup of dried PSL spheres collecting between the spherical impactors and the nozzles. A more diluted PSL solution that still produced acceptable seed cloud density would have solved this problem. For reasons which will follow, work with the Six-Jet Atomizer was abandoned before this ideal mixture was found.

The TSI Six-Jet Atomizer successfully produced a dry seed cloud that minimized impaction on the walls and ceiling of the plenum chamber, and the advertised flowrate of 6.5 liters per minute was achieved, according to approximate measurements. When injected into the tunnel, the seed particles made the laser beams visible; however, the beams were faint even when the unit was in peak operation. Clearly, a higher volume of seed was required, the solution to which would be the purchase or construction of an additional six-jet unit. This was prevented by exorbitant price in the former case, and difficulty of manufacturing in the latter. The important lesson learned from the testing of this device was that the impactor was a critical component to preventing large droplets from exiting the atomizer and producing a fastdrying seed aerosol.

\section{C.3.4 Laskin Nozzle}

The Laskin nozzle aerosol generator that is used in dioctyl phthalate (DOP) seed generation was tested with PSL solution. The aerosol generator was tested with a variety of compressed air pressures, PSL liquid mixtures, and number of operational nozzles, but little success was had. Though the exit flowrate was high, the aerosol consisted mainly of purely liquid droplets that evaporated quickly, leaving behind no noticeable PSL seed cloud. It is unknown what the average ethanol or ethanol/water droplet size was, but the average DOP droplet produced is $0.7 \mu \mathrm{m}$ in diameter. Hence, the droplet formed may not be large enough to contain a PSL sphere. A solution of 2.0 
$\mu m$ spheres was replaced by a solution of $1.11 \mu m$ spheres, but no noticeable difference resulted. A drop of liquid soap was added to the PSL solution to see if changes in surface tension produced any effect, but the only outcome was a frothing of the PSL liquid solution and a froth/liquid mix exiting the aerosol outlet.

The Laskin nozzle used in this study is designed for use with DOP, so it is thought that PSL particle size, and the properties (such as surface tension) of the ethanol and/or water prevented it from working properly for PSL atomization. The lack of favorable results in testing of the Laskin nozzle seeder, along with information from Mazumder et al. (1975) showing superior performance of ultrasonic nozzles led to the investigation of other aerosol production options.

\section{C.3.5 Ultrasonic Nozzles}

As an alternative to the Spray Systems nozzles discussed above, which were never able to produce fine enough droplets for this application, the use of ultrasonic nozzles was investigated. Ultrasonic nozzles generate a highfrequency sonic field when compressed air is accelerated into a hollow resonator chamber (Figure C.9). A stream of liquid (PSL solution in this case) is introduced into the resonator chamber, where the sound waves atomize the liquid into a fine mist.

The droplet diameter for the nozzles considered (Sonimist 700 series) is on the order of 10-25 $\mu \mathrm{m}$ in diameter (Gajnos; Mis, 2002), based on tests done with water. This size can be varied by changing the frequency of the sound waves, the spacing between the resonator chamber and the air orifice, and varying the ratio of gas to liquid flow. The nozzles can atomize about 40 to $375 \mathrm{ml} / \mathrm{min}$ of liquid. A representative seed solution of half ethanol and half PSL solution has a concentration, $C_{\text {soln }}$, of $2.4 \mathrm{e} 10$ particles $/ \mathrm{mL}$. Choosing a conservative liquid flowrate, $Q_{l i q}$, of $200 \mathrm{ml} / \mathrm{min}$, a nozzle produces,

$$
r=C_{\text {soln }} Q_{\text {liq }}=4.8 \mathrm{e} 12 \text { particle } / \text { min }
$$

Thus, these calculations predict that two ultrasonic nozzles could produce a sufficient amount of seeding for this application. Implementation would be relatively easy, in that these nozzles could replace the Spray Systems nozzles 


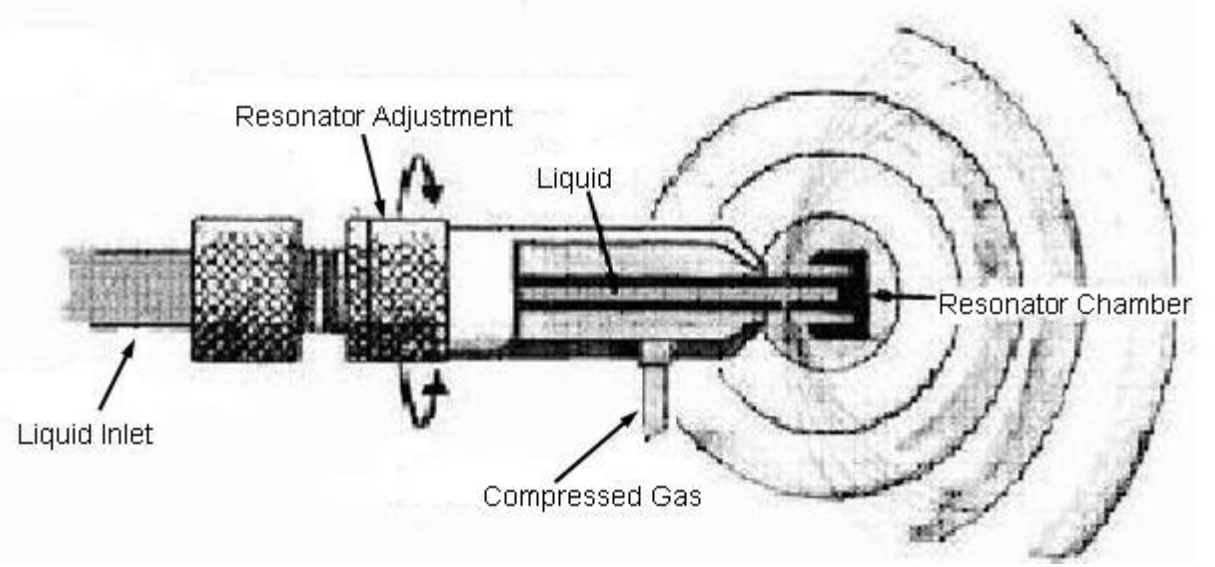

Figure C.9: Schematic of ultrasonic nozzle. Ref. Mis (2002)

in the apparatus previously described. Injection into the tunnel remains a problem, and the most likely solution being that the nozzles would need to be installed inside the plenum of the wind tunnel. This arrangement would have the added inconvenience necessitating occasional cleaning, being that this location is upstream of the turbulence screens and honeycomb. A similar setup using an array of airbrushes was used successfully by Seegmiller (1985) in an open jet tunnel with comparable test section size and flow speed, though the rate of seed production is not documented. In the present study, the option of ultrasonic nozzles was never fully investigated because of the excessive cost of the nozzles (approximately $\$ 1600$ each) and the amount of undiluted seed solution that would be consumed (about 3 gallons for every hour used).

\section{C.3.6 Medical Nebulizers}

Medical nebulizers produce a fine aerosol that requires very little drying, making them an ideal choice for this application. Nebulizers have been used previously to produce PSL aerosols, but usually in applications that require much lower amounts of seed particles (Adams et al., 1985; Chang and Okuyama, 2002). Though the aerosol quality is very good, a single nebulizer has a low output flowrate. This required the development of an apparatus that could continuously operate multiple nebulizers. 


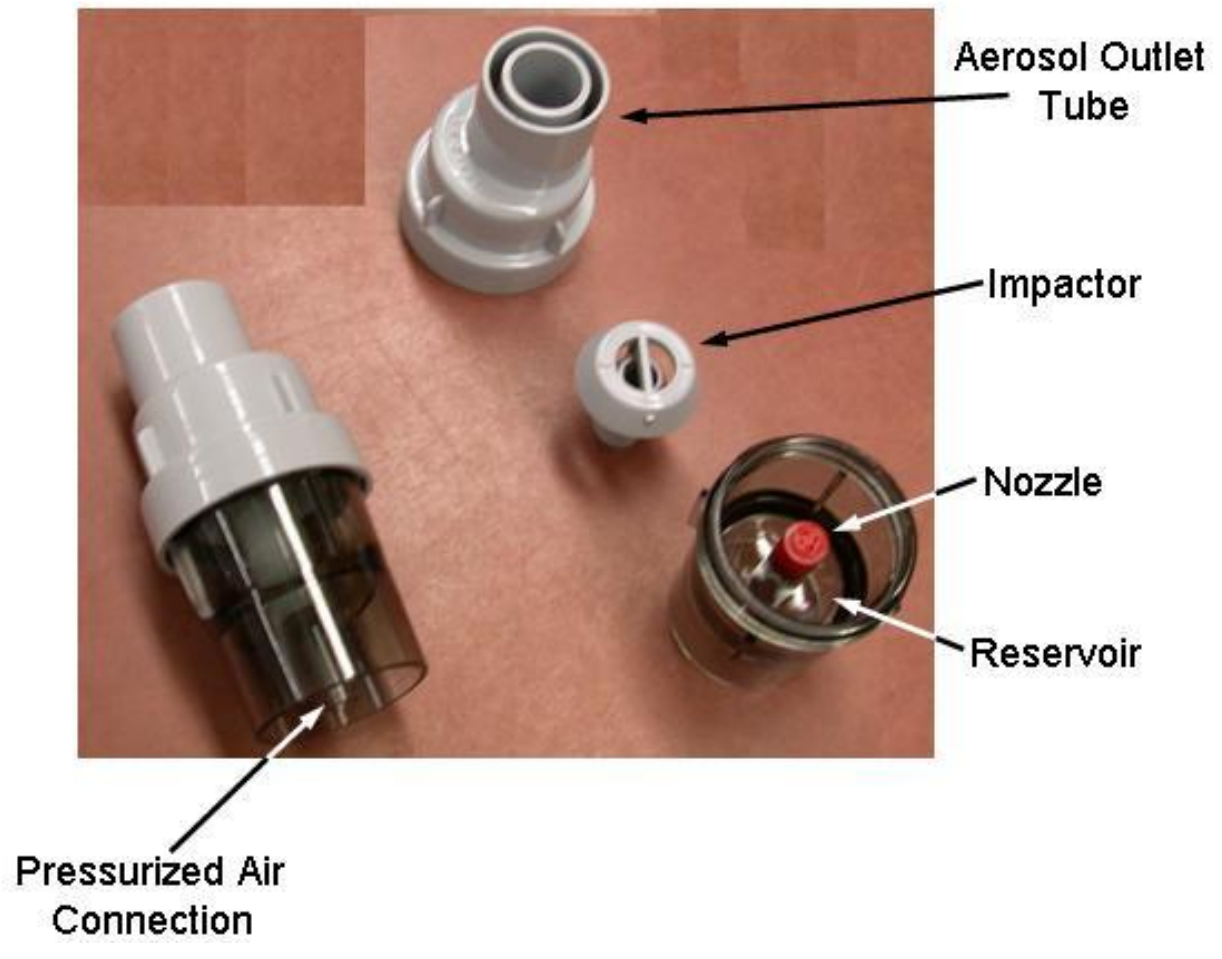

Figure C.10: Invacare Sidestream nebulizer. Left - Assembled nebulizer. Right - Nebulizer parts. 
In this investigation, Invacare Sidestream nebulizers (pictured in Figure C.10) were used; they were chosen because they nebulized a given volume of liquid faster as compared to ten other commercially available units that were tested. The nebulizers operate by drawing liquid up from the reservoir to the nozzle by the pressure drop caused by a jet of compressed air. The aerosolized liquid impacts on a baffle plate, eliminating large droplets. The aerosol then exits through a orifice at the top of the unit. The output flowrate versus supply air pressure of the Sidestream nebulizer was characterized with a pitot probe and is plotted in Figure C.11. Volumetric nebulization rate versus supply pressure is plotted in Figure C.12. Based on these tests, an expected seed density in the tunnel can be calculated, using the representative seed solution mentioned in the previous section (half ethanol and half PSL solution with a concentration, $C_{\text {soln }}$, of $2.4 \mathrm{e} 10$ particles $/ \mathrm{mL}$ ). At 40 PSI, the flowrate out of the nebulizer is $23.8 \mathrm{Lpm}$, and the nebulization rate, $Q_{n e b}$, is 0.75 $\mathrm{mL} / \mathrm{min}$. The flowrate of the boundary layer in the wind tunnel, $Q_{B L}$, is 12.7 $\mathrm{m}^{3} / \mathrm{min}$.

$$
\begin{aligned}
r_{n e b} & =Q_{n e b} C_{\text {soln }}=1.80 e 10 \text { particle } / \text { min } \\
C_{B L, 1} & =r_{n e b} / Q_{B L}=1.42 e 9 \text { particles } / \mathrm{m}^{3} \\
& =1.42 e 3 \text { particles } / \text { cc }
\end{aligned}
$$

Where $C_{B L, 1}$ is the concentration of the particles in the boundary layer due to one nebulizer.

To operate multiple nebulizers simultaneously, an apparatus was designed which arranged multiple nebulizers in a platform that would then float in a reservoir of PSL solution. First, ten nebulizers were modified by drilling liquid "feed holes" into their reservoirs. The nebulizers are fixed securely in a thin, circular metal plate, which is then attached to a $1.25 \mathrm{~cm}$-thick foam disc that has holes into which the nebulizers fit, forming a float (Figure C.13). When placed into a basin, this float holds the nebulizers such that their reservoirs and liquid feed holes are submerged, while the aerosol output tubes are still above the liquid level. The basin used is a 23-quart pressure canner which is filled to some level with the PSL seed solution. The canner has a lid with a gasket which creates an airtight seal. The compressed supply air enters the canner through a connection in this lid (Figure C.14). Mounted 


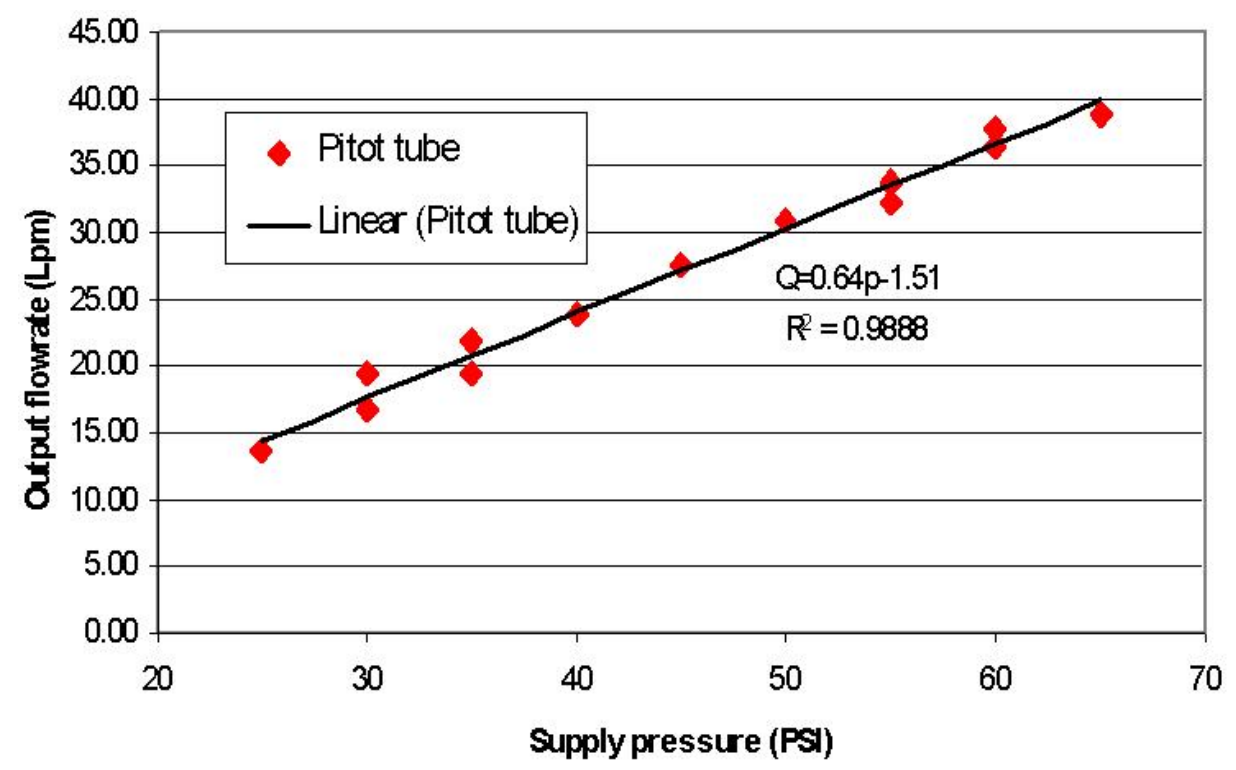

Figure C.11: Supply pressure vs. nebulizer output flowrate. Based on pitot probe measurements at mouth of nebulizer.

on the underside of the lid is a manifold which distributes the compressed air into the ten tubes that in turn pass through a hole in the center of the float and connect to the nebulizers. Also mounted in the lid is a connection for a water hose through which the aerosol exits and a second air connection to provide diluting air if it is required.

This apparatus was tested by connecting its output via a manifold to three of the central inlet holes under the baffle plate. The mixture used was equal parts of ethanol and PSL solution $(1.11 \mu \mathrm{m}$ diameter, approximately 6e10 particle $/ \mathrm{mL}$ ). The PSL seeder was run at a variety of supply and co-flowing air pressures.

A 3-component LDV system was used to characterize the particle loading in the wind tunnel. The $55 \mu \mathrm{m}$ diameter measurement area was placed 545 $\mu \mathrm{m}$ from the wall, corresponding to a mean streamwise velocity component of $13.8 \mathrm{~m} / \mathrm{s}$. The data rate for one component of the LDV system was 40-50 bursts/s, while the 3 component coincident data rate was 10-15 bursts/s. These values did not vary appreciably with changes in supply or diluting air 


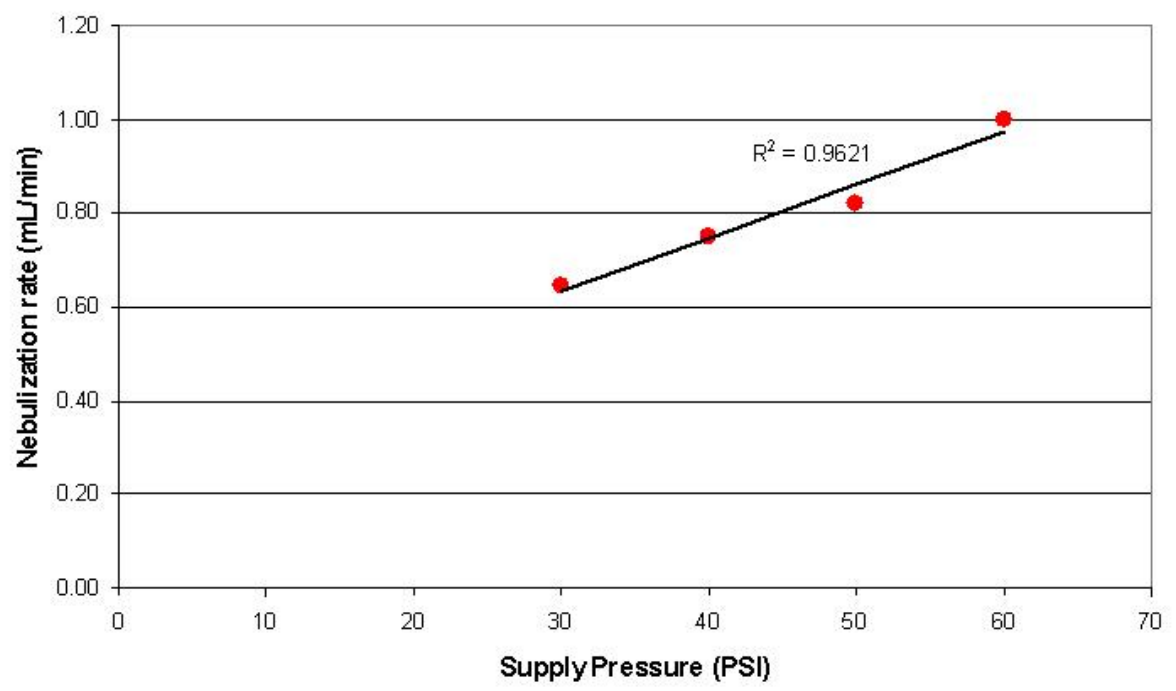

Figure C.12: Nebulization rate vs. supply pressure. Based on measurements of time to nebulize $10 \mathrm{~mL}$ of water.

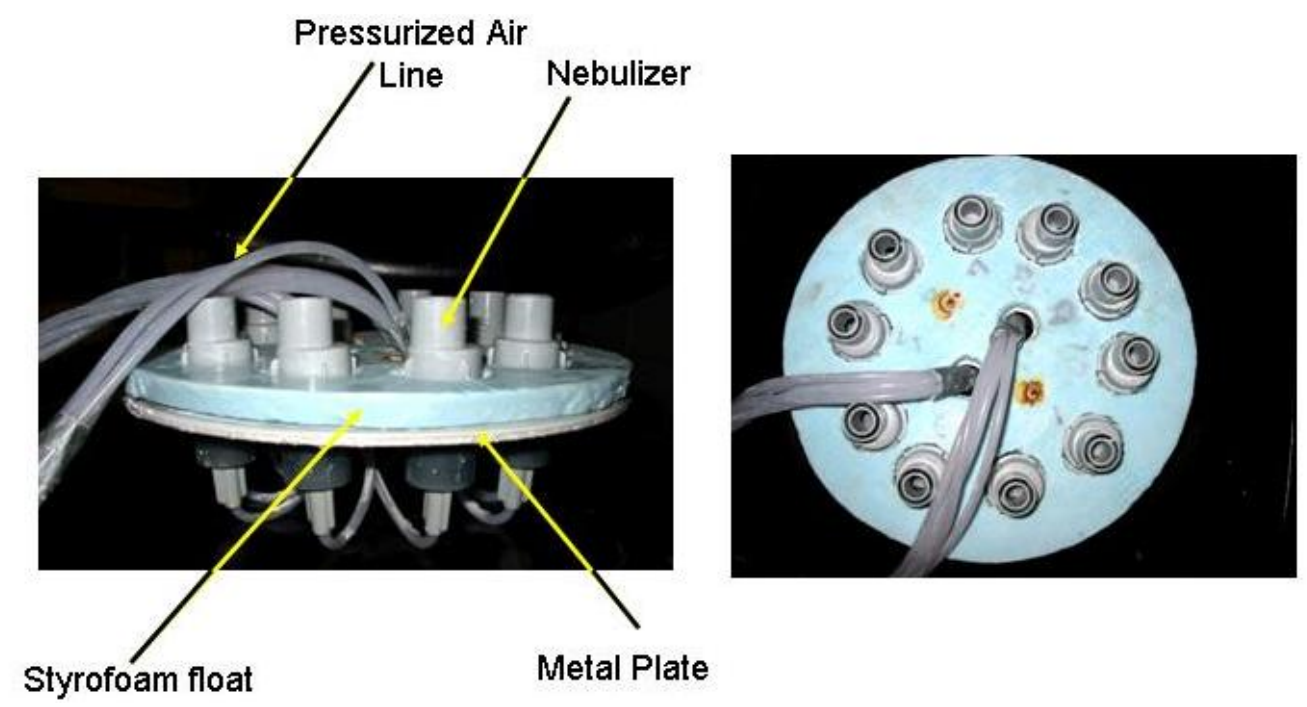

Figure C.13: Nebulizer float. Left - Side view. Right - Top view. 


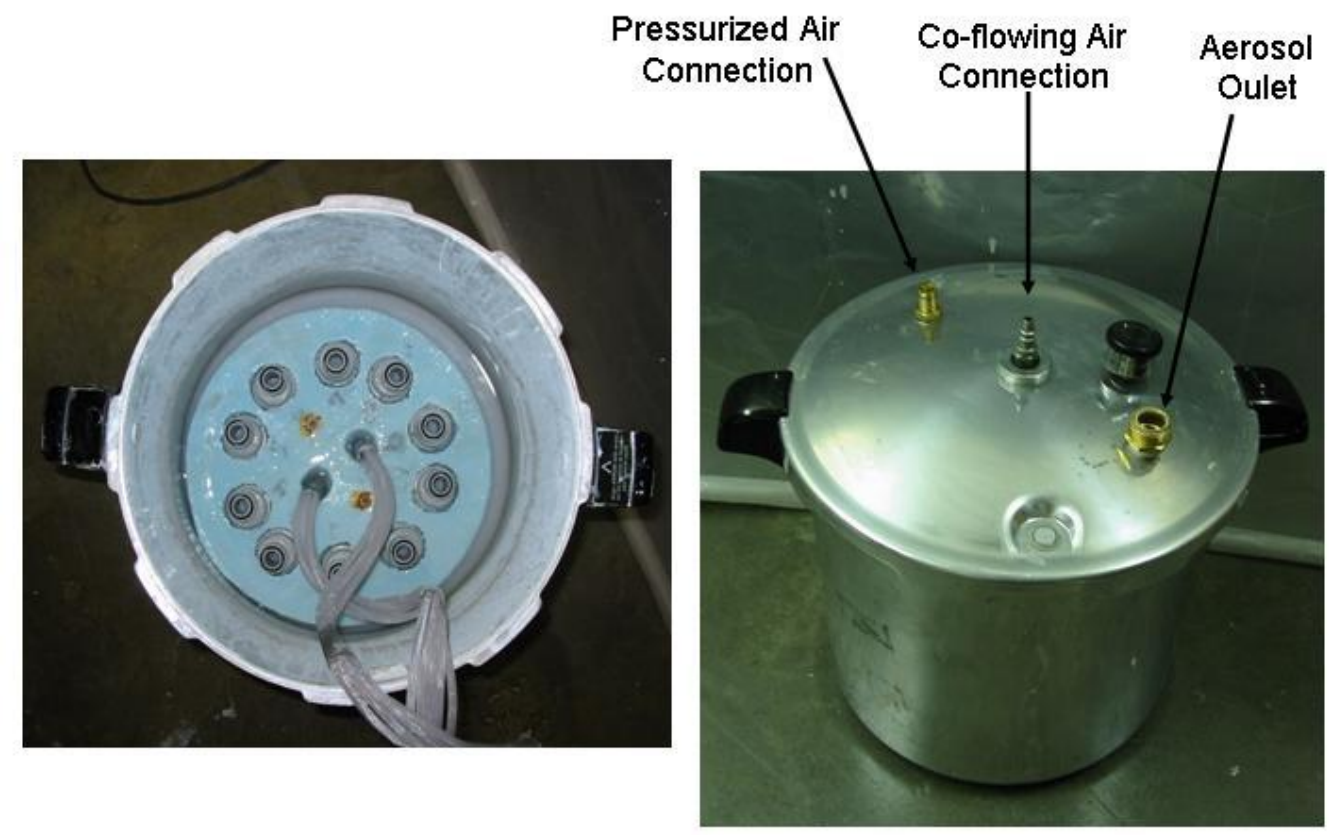

Figure C.14: Nebulizer seeder. Left - Top view with lid removed. Right Assembled seeder. 
pressure. Assuming a measurement volume diameter of $55 \mu \mathrm{m}$,

$$
Q_{M V}=A_{M V} \cdot U=0.038 \mathrm{~cm}^{3} / \mathrm{s}
$$

Where $A$ is the cross sectional area of the measurement volume, $U$ is the streamwise velocity component (perpendicular to $A$ ), and $Q_{M V}$ is the flowrate through the measurement volume.

Using both the one component and three component coincident data rates, seed concentration are calculated,

$$
\begin{aligned}
C_{1 \text { comp }} & =45 \text { particles } / \mathrm{s} / Q_{M V}=1.18 \mathrm{e} 3 \text { particles } / \mathrm{cc} \\
C_{\text {coincident }} & =10 \text { particles } / \mathrm{s} / Q_{M V}=0.31 \mathrm{e} 3 \text { particles } / \mathrm{cc}
\end{aligned}
$$

This calculation assumes that all particles present in the measurement volume cause a validated burst, so the actual concentration is slightly higher than these estimates. But even the most optimistic interpretation of these values falls far short of the prediction above $\left(C_{B L, 1} \cdot 10=1.42 \mathrm{e} 4\right.$ particles $\left./ \mathrm{cc}\right)$. This difference is probably due in part to the some seed particles settling out of the liquid solution or impaction of seed particles in the canner, tubing and baffle plate. The former is probably more significant, since at such high PSL solution concentrations, a thick layer of solid tends to form on the bottom of the canner.

It was observed with the nebulizers as well as with the six-jet atomizer that high particle concentrations tend to clog the nozzles. This effect was also noted by Meyers (1991). The high particle concentration solutions used in an attempt to meet the high seeding requirements may not be feasible with nozzles that have very small openings or impactors very near the nozzle

exit, producing another upper limit on the amount of PSL seed that can be produced.

\section{C.4 DOP Seeding with a Vap/Con Aerosol Generator}

As mentioned previously, in order to yield better comprehensive LDV data, a monodisperse seed aerosol is necessary. The di-octyl phthalate (DOP) 


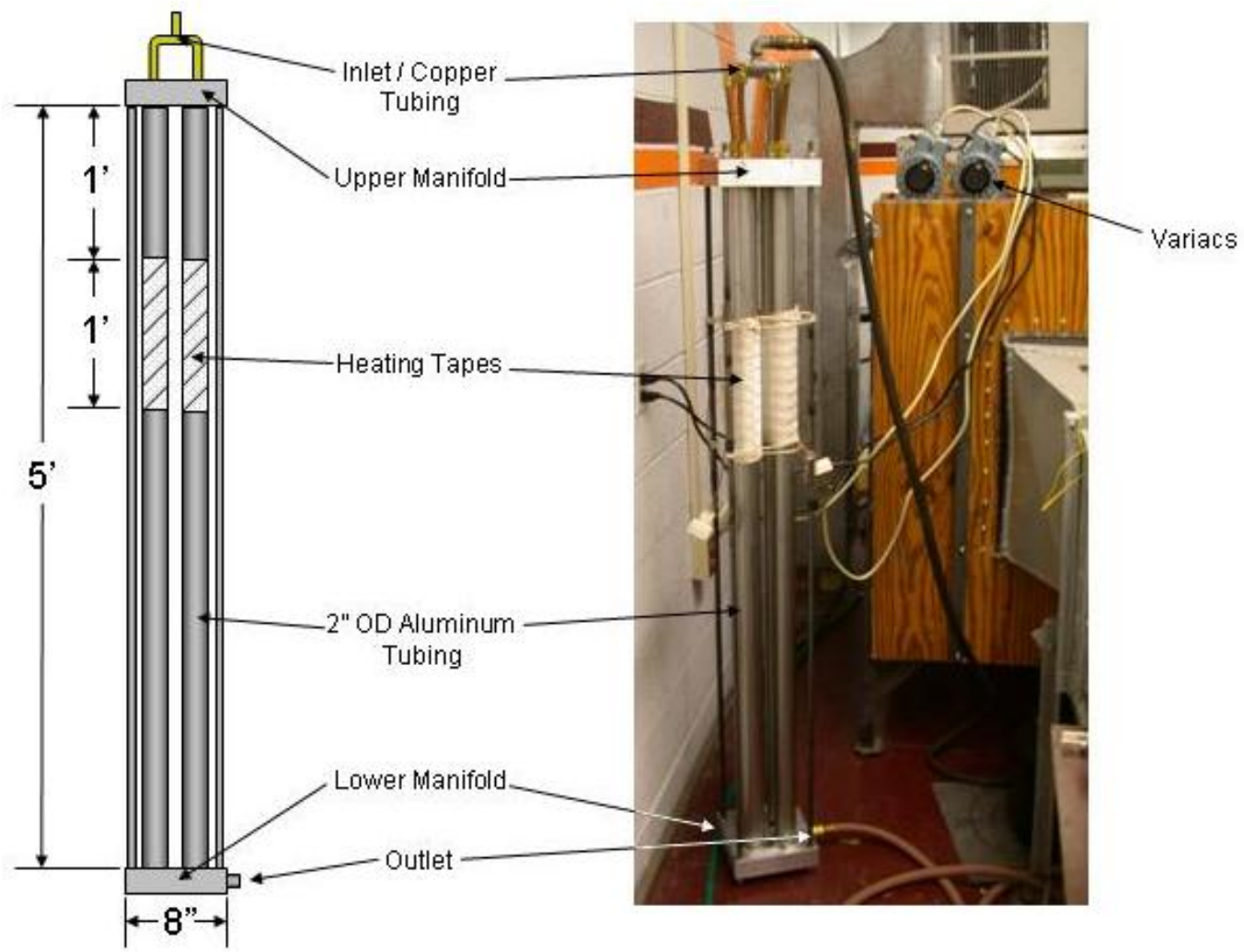

Figure C.15: Vap/con Generator Schematic and Photograph. Note that insulation is not present on heat tapes.

generator produces a polydisperse aerosol, so a vaporization/condensation (vap/con) aerosol generator is utilized to produce more consistently-sized seed particles. Small seed particles appear as noise, and large particles don't follow the flow well, so elimination of these particles improves the LDV data taken in the seeded wind tunnel flow.

The vap/con generator is based on a design originally by Liu et al. (1966). The current design, adapted from that of Yanta (1974), is pictured in Figure C.15. The vap/con generator vaporizes the DOP aerosol as it passes through heated tubes. The DOP then recondenses on condensation nuclei, or nonvolatile impurities. The recondensed particles have a much more homogenous sizing than in the original aerosol.

The concept of generation of monodisperse aerosols by condensation was 
first described by LaMer and Sinclair (1943). The Sinclair-LaMer apparatus, as well as later, improved designs (Muir, 1965), had several disadvantages, including poor reproducibility and a long time to reach steady state operating condition. The Rapaport-Weinstock generator (Fuchs and Sutugin, 1966), from which the apparatus described here is derived, solved these problems. This type of aerosol generator has very reproducible results. Since one liquid drop is formed per condensation nuclei, particle size is a function of the ratio of aerosol material to impurities. Since this ratio is relatively constant, so is the resulting size of the droplets produced by the vap/con generator (Liu et al., 1966). This type of generator reaches operating conditions on the order of 10 minutes, rather than hours. Temperature does not have to be held rigorously constant, as in the Sinclair-LaMer generator, since after evaporation of the primary droplets is achieved, the vapor concentration is independent of temperature (Fuchs and Sutugin, 1966).

\section{C.4.1 Design Details}

The main structure consists of 4 stainless steel tubes $1.52 \mathrm{~m}$ in length with an outer diameter of $5.08 \mathrm{~cm}$ and a wall thickness of $0.165 \mathrm{~cm}$. They are connected at top and bottom by aluminum manifolds measuring $5.08 \mathrm{~cm}$ thick and 20.3 by $20.3 \mathrm{~cm}$. These manifolds also double as heat sinks as the apparatus heats up. The incoming aerosol is divided into the four steel tubes by copper tubes that enter the upper manifold through standard pipe fittings (Baker, 1986).

Four HTS/Amptek heavy insulated heating tapes are the heat sources to vaporize the DOP. The tapes are fiberglass insulated and are $183 \mathrm{~cm}$ long by $2.54 \mathrm{~cm}$ wide. At their maximum voltage of $120 \mathrm{~V}$, they supply $624 \mathrm{~W}$ each to the aluminum tubes. The Amox insulation on the tapes can withstand temperatures up to $760{ }^{\circ} \mathrm{C}$. The heat tapes are wrapped around the steel tubes starting approximately $30.5 \mathrm{~cm}$ below the upper manifold and cover approximately $30.5 \mathrm{~cm}$ of the length of the steel tubes. The heating tapes are in turn wrapped in two layers of fiberglass insulation, which, at peak operation, increases the temperature on the tubes by about $93{ }^{\circ} \mathrm{C}$. The four heating tapes are plugged into two power strips with a maximum current of $15 \mathrm{~A}$ and a rated input voltage of $120 \mathrm{~V}$. The power strips are powered by two variable transformers with a maximum output voltage of $140 \mathrm{~V}$. The 
variable transformers can withstand a maximum of $10 \mathrm{~A}$, and the heating tapes draw 5.2 A at their maximum voltage of $120 \mathrm{~V}$. Thus, with two heating strips in each variable transformer, care must be taken to not exceed their 10 A limit.

The boiling point of DOP is $231{ }^{\circ} \mathrm{C}$, so the challenge of this system is finding a voltage level that provides a sufficient level of heat to vaporize all the DOP at a given flow rate of the air/seed mixture. This flow rate is determined by the amount of seed necessary for wind tunnel measurements. Obviously the tapes will have to be set at a considerably higher temperature than $231^{\circ} \mathrm{C}$ in order to heat the entire flow through the tubes. Estimates for this temperature range from $288^{\circ} \mathrm{C}$ to $427^{\circ} \mathrm{C}$ (Baker, 1986). On the other hand, too high of a heating tape temperature may result in an increased degree of superheat of the DOP, which would destroy the homogeneity of the particle size (Liu et al., 1966).

To err on the side of caution, the DOP generator is operated at the high end of the suggested temperature range, to help ensure all DOP is vaporized. The DOP generator is run at 18 psi, and the heating tapes achieve 427 to $432{ }^{\circ} \mathrm{C}$ at about $100 \mathrm{~V}$. Temperature is measured by placing a thermocouple probe between the heating tape and the tube.

\section{C.4.2 Results}

Figure C.16 is a representative time series of the LDV signal with and without the vap/con operating. Visual inspection yields qualitative evidence that the signal has improved. The bursts due to particles in the measurement volume appear more regularly sized when the vap/con is employed, indicating increased homogeneity. Noise due to small particles also appears to be decreased. Validation of bursts also improves with the vap/con; out of 100,000 bursts, 49,234 were validated with the vap/con, versus only 37,007 without it. This represents a 33 percent improvement.

These improved results were confirmed more rigorously with a TSI Aerodynamic Particle Sizer (APS) spectrometer. The aerosol produced by the Laskin nozzle system with the vap/con has a geometric standard deviation of approximately $22 \%$ (geometric standard deviation quantifies the variation of particle sizes). At the same operating conditions, but with the vap/con sys- 

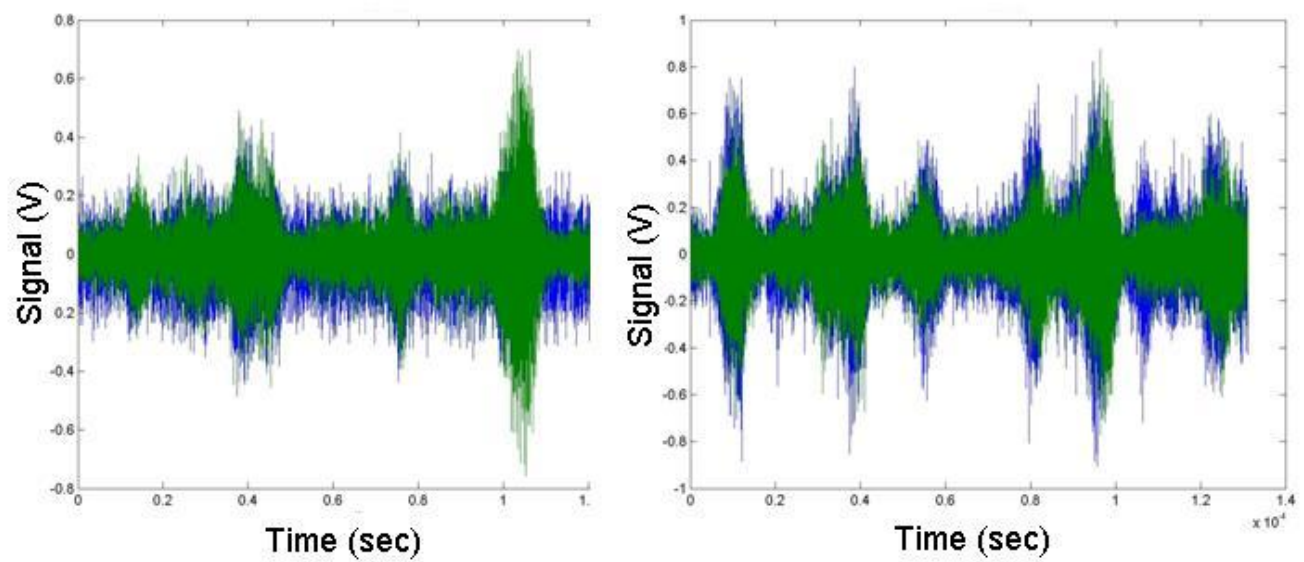

Figure C.16: Left - LDV signal without vap/con. Right - Signal with vap/con. Note more regularly sized bursts, and fewer smaller, noisy bursts.

tem operating, the geometric standard deviation is reduced to about $11 \%$. In addition, the system with the vap/con has $55 \%$ fewer small, noise-producing particles than the system without the vap/con. The amount of seed produced is not affected by the presence of the vap/con, and is sufficient to yield an excellent LDV data rate. These results show a very significant improvement in the seeding system due to the presence of the vap/con aerosol generator.

\section{C.5 Conclusions}

Attempts to seed the Virginia Tech Small Boundary Layer Wind Tunnel with PSL spheres were met with limited success. Low amounts of seed were produced and injected into the tunnel, but not in sufficient quantity to meet the high particle concentration required here.

Lack of success was a result of the specific requirements of this wind tunnel. In order to prevent clogging the turbulence screens, the PSLs were injected through a baffle plate in the contraction section just upstream from the test section. Because of the short drying distance for the seed aerosol, it was required that a very dry seed was produced. Unfortunately, the methods which accomplish this (six-jet atomizer, medical nebulizer) have a low output flow rate. In the case of the medical nebulizers, this problem was attempted 
to be overcome by increasing the number of units as well as the concentration of the solution. However, even at reasonable upper limits of the number of units operating, not enough seed would be produced, and increasing particle concentration only promotes agglomeration of the particles and clogging of the nozzles.

For these research purposes, it was determined that the degree of increase in the monodispersity of the DOP particles provided by a Vaporization/Condensation Aerosol Generator, along with signal processing improvements, would be sufficient. Amount of seed has been determined to be the critical factor, even at the sacrifice of seed quality; no system, as of yet, seems to be able to match the aerosol production of the Laskin nozzle generator with DOP.

PSL seeding remains a viable option for certain applications where seed quality is more important than quantity. The medical nebulizer apparatus could be successfully employed and produce a moderate amount of seed, especially with multiple units; three units identical to that described above (a total of thirty nebulizers) could be attached under the baffle plate.

For higher seed production, the option of ultrasonic nozzles could still be fully investigated, if the need warranted the cost. They have the potential to produce a large volume of seed with very small (therefore fast-drying) droplets. Due to the large amount of PSL solution the nozzles may consume, it would be recommended that in-house production of PSLs be investigated. They can be produced for less than $\$ 1$ per liter with some basic equipment (Nichols, 1987). In the case of the Small Boundary Layer Tunnel, these nozzles would probably have to be placed in the plenum section, and modifications to the tunnel would have to be made for access to the turbulence screens for cleaning purposes. 


\section{Vita}

Devin Stewart attended the University of Maryland, College Park, from which he received his B.S. in Aerospace Engineering and his B.A. in Spanish Language and Literature in May of 2001. In the fall of 2001, Devin traveled to Madrid, Spain, where he worked as a teacher of English as a Foreign Language. Devin earned his Master's degree in Aerospace Engineering in the fall of 2005 from Virginia Polytechnic Institute and State University. In the summer of 2005, he was a Science and Technology Graduate Policy Fellow with the National Academies in Washington, D.C. 